U.S. Department of Energy
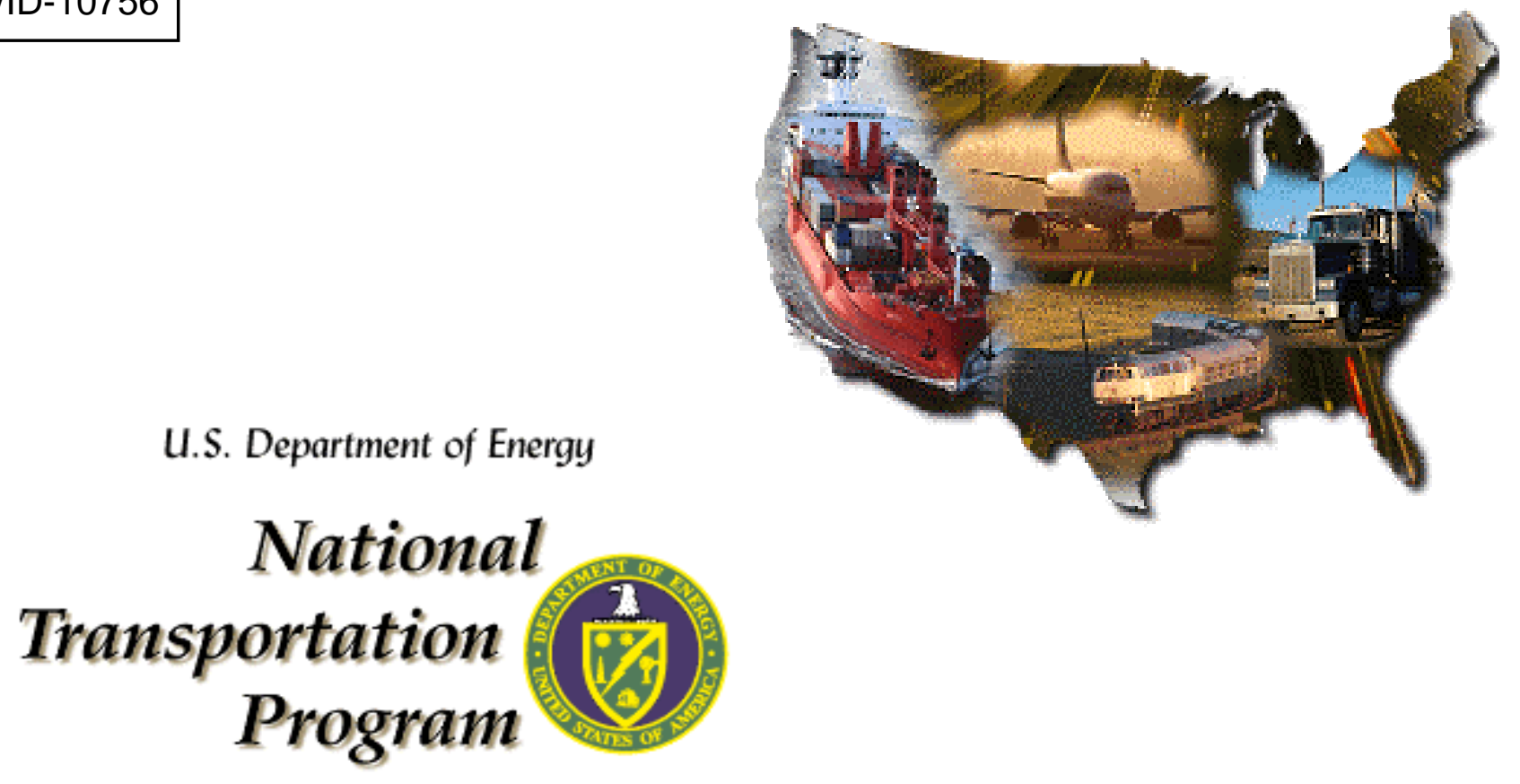

\title{
TRANSPORTATION BASELINE SCHEDULE
}

Prepared by:

National Transportation Program Integration and Planning Bechtel BWXT Idaho, LLC

Idaho National Engineering and Environmental Laboratory P.O. Box 1625

Idaho Falls, ID 83415-1625

January 11, 2000

Rev. 01 



\section{Table of Contents}

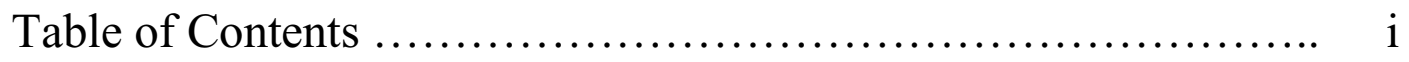

Introduction ................................................ 1

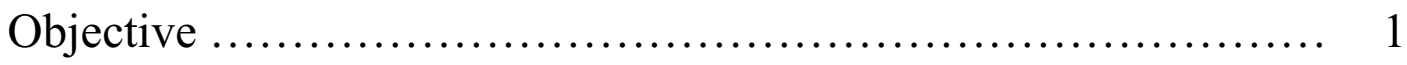

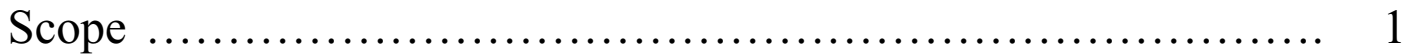

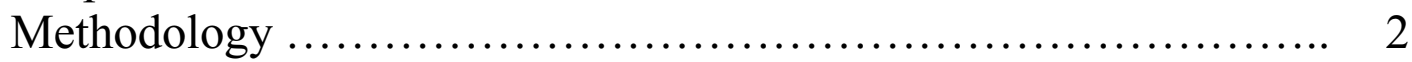

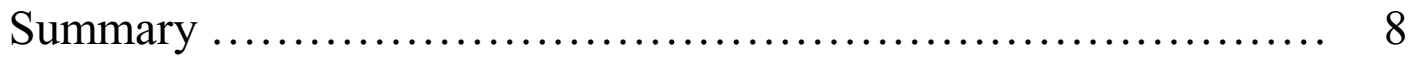

Abbreviations and Geographical Site Acronym List ............... 9

Glossary .................................................... 11

Appendix A 1999 Transportation Baseline Schedule Maps and Number of Shipments/Volume Graphs ..... A-i

Fernald Life-cycle Outgoing Map $\ldots \ldots \ldots \ldots \ldots \ldots \ldots \ldots \ldots \ldots \ldots$ A-1

Hanford Life-cycle Outgoing Map .......................... A-5

Hanford Life-cycle Incoming Map ......................... A-11

INEEL Life-cycle Outgoing Map $\ldots \ldots \ldots \ldots \ldots \ldots \ldots \ldots \ldots \ldots \ldots$ A-2 1

INEEL Life-cycle Incoming Map .......................... A-31

Los Alamos Life-cycle Outgoing Map ....................... A-41

Los Alamos Life-cycle Incoming Map ...................... A-47

Nevada Life-cycle Outgoing Map ........................... A-51

Nevada Test Site Life-cycle Incoming Map .................... A-55

Nevada Test Site Life-cycle potential Incoming Map ............ A-63

Oak Ridge Life-cycle Outgoing Map ......................... A-67

Oak Ridge Life-cycle Incoming Map ...................... A-75

Rocky Flats Life-cycle Outgoing Map ...................... A-81

Sandia National Laboratory Life-cycle Outgoing Map ........... A-87

Savannah River Site Life-cycle Outgoing Map .................. A-95

Savannah River Site Life-cycle Incoming Map .................. A-103

West Valley Life-cycle Outgoing Map ......................... A-107

WIPP Life-cycle Incoming Map ............................ A-115

Geologic Repository Disposal Life-cycle Incoming Map ......... A-125 



\section{National Transportation Program 1999 Transportation Baseline Schedule}

\subsection{Introduction}

The "1999 National Transportation Program - Transportation Baseline Report" presents data that form a baseline to enable analysis and planning for future Department of Energy (DOE) Environmental Management (EM) waste/material transportation. The companion "1999 Transportation 'Barriers' Analysis" analyzes the data and identifies existing and potential problems that may prevent or delay transportation activities based on the data presented. The "1999 Transportation Baseline Schedule" (this report) uses the same data to provide an overview of the transportation activities of DOE EM waste/materials.

This report can be used to identify areas where stakeholder interface is needed, and to communicate to stakeholders the quantity/schedule of shipments going through their area. Potential bottlenecks in the transportation system can be identified; the number of packages needed, and the capacity needed at receiving facilities can be planned. This report offers a visualization of baseline DOE EM transportation activities for the 11 major sites and the "Geologic Repository Disposal" site (GRD).

\subsection{Objective}

The objective of this report is to provide an overview of the planned transportation activities for DOE EM waste and materials as reported in the FY 1999 Integrated Planning, Accountability, and Budgeting System (IPABS) Stream Disposition Data (SDD).

\subsection{Scope}

This report gives a graphical view of planned transportation activities for the 11 major DOE EM sites and for the GRD (tentatively Yucca Mountain). For sites that may act as repositories, (i.e., Hanford, Nevada Test Site (NTS), WIPP, and Yucca Mountain for this report) all incoming shipment data is included (e.g., shipments from small sites). The 11 major sites included are:

Fernald Environmental Management Project

Hanford Site

Idaho National Engineering and Environmental Laboratory

Los Alamos National Laboratory

Nevada Test Site 
Oak Ridge Reservation

Rocky Flats Environmental Technology Site

Sandia National Laboratory

Savannah River Site

Waste Isolation Pilot Plant

West Valley Demonstration Project

Data presented is from the 7/30/99 version of the "Stream Disposition Data" (SDD) captured in IPABS. It includes information on Mixed/Low Level Waste (M/LLW), Transuranic (TRU), High Level Waste (HLW), and Spent Nuclear Fuel (SNF) waste/material streams. Data used for the Geologic Repository Disposal map includes information collected in conjunction with the Integrated Repository Receipts Workshop conducted during FY 1999. This report focuses on the number of shipments. However, volume schedule data are presented where number of shipments information was not available.

\subsection{Methodology}

One of the transportation related data elements captured in IPABS is "Number of Shipments." The following is the definition for "Number of Shipments" from IPABS:

- \# of Shipments is the number of shipments during the reporting period that are planned for the disposition quantity of the stream that is within the specified combination of Material Category, Package Type/Capacity, and Mode. For the rail mode, each rail car is considered a shipment. For the truck mode, a single trailer is considered a shipment.

The "Number of Shipments" data element was entered into IPABS by each site. It is not derived in from waste/material volumes.

For each waste/material stream the annual number of shipments were reported. For this report, those numbers were summed to obtain the life-cycle number of shipments for each origin - destination pair. 
Figure 1 is an example of a 1999 Transportation Baseline Schedule map (shown full size in Appendix A). It is a high level view of incoming/outgoing shipments for the specified site with the accompanying origin/destination.

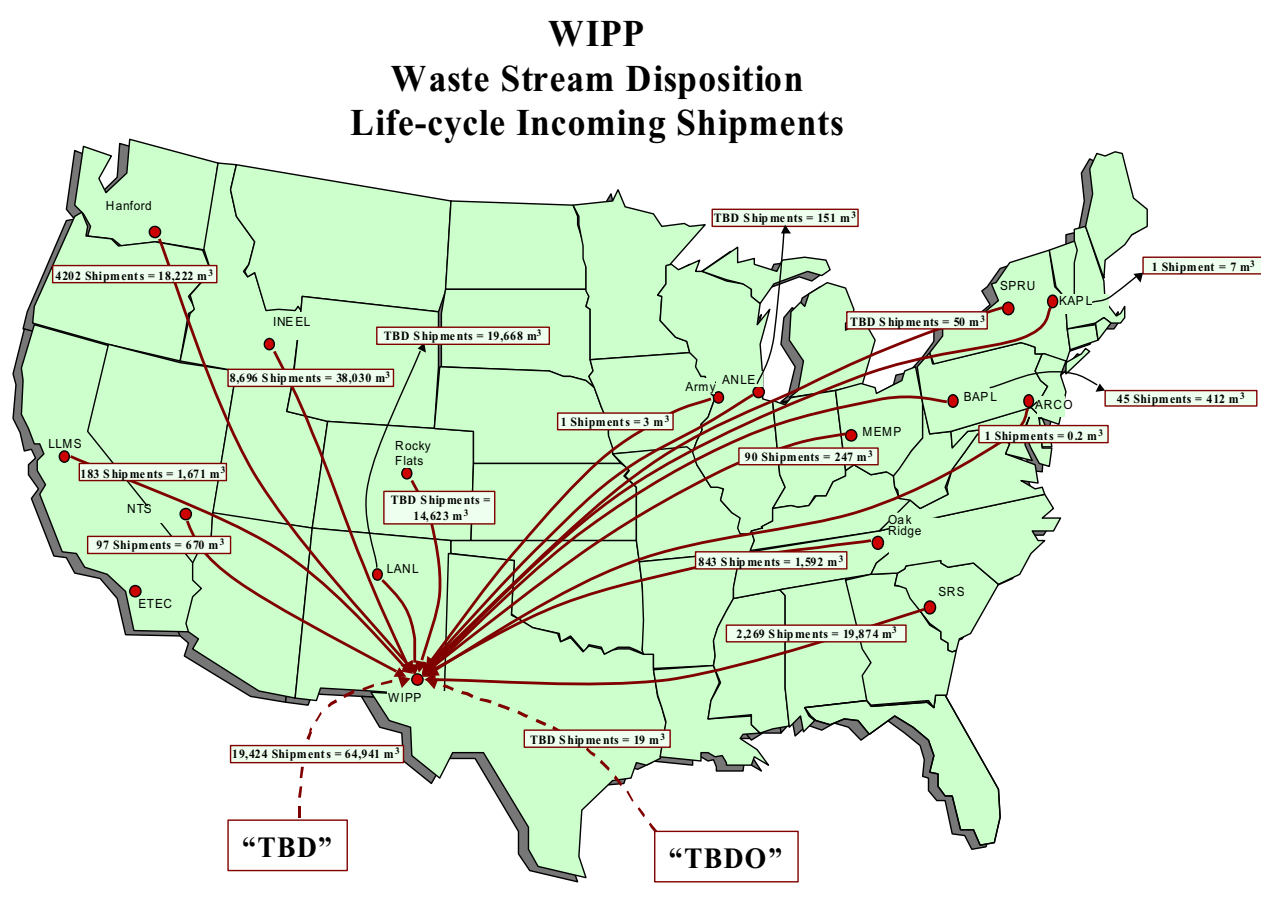

Figure 1. Transportation Baseline Schedule Map.

An information box appears on (or near) the red arrow for each origin-destination pair. The total life-cycle number of shipments from 7/30/99 SDD in IPABS is indicated for each site with its accompanying volume (Figure 2).

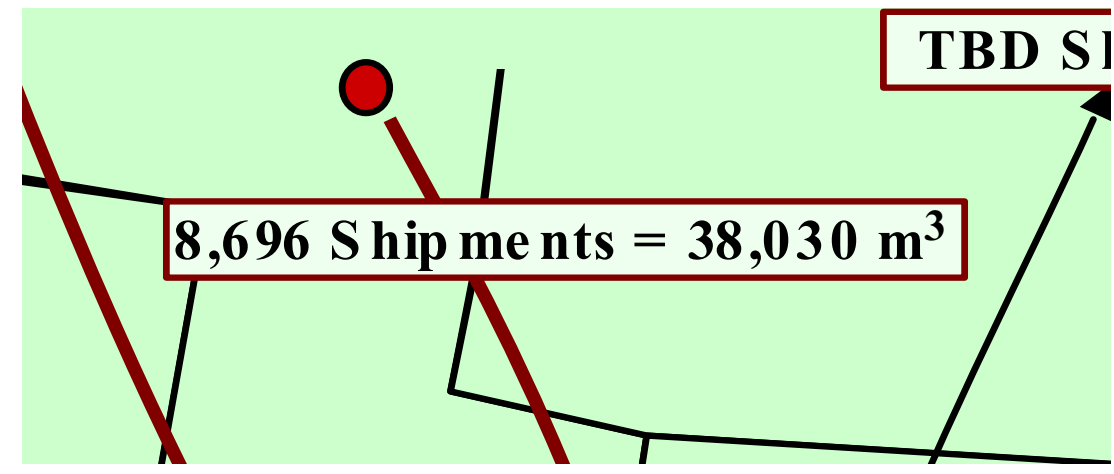

Figure 2. Total life-cycle number of shipments and associated volume as reported in IPABS SDD. 
If a waste/material stream volume is present in the SDD with no shipping information, it is indicated as "TBD Shipments." Figure 3 is an example of a baseline schedule map containing a volume with no corresponding shipping information.

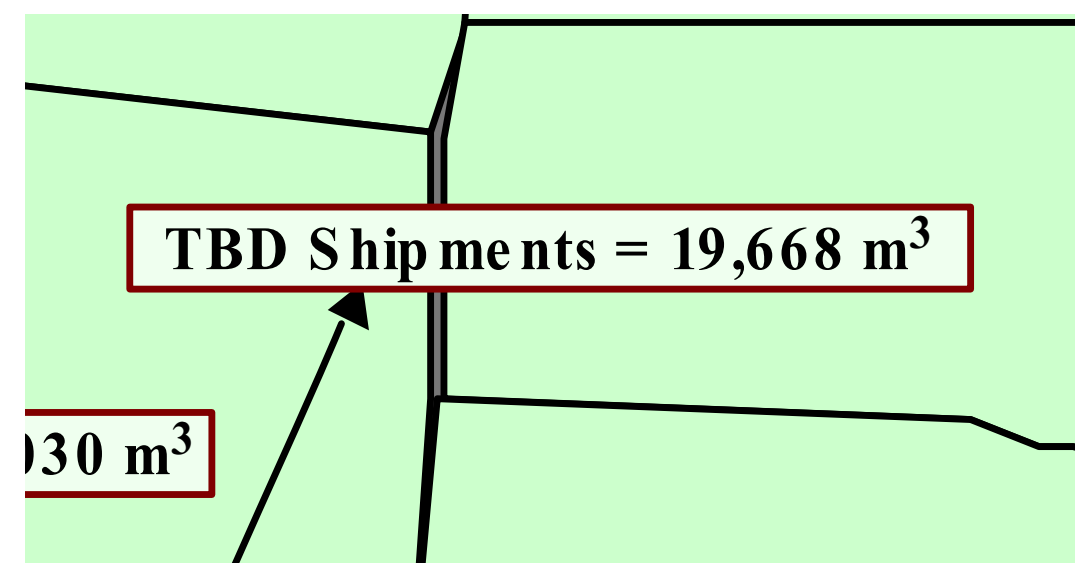

Figure 3. Volume reported in IPABS SDD with no shipping information.

If some of the waste/material has shipping information in IPABS SDD for a given origin - destination pair and others do not, both are displayed in the information box. Figure 4 is an example of an origin-destination pair where the SDD contains the number of shipments for $38,030 \mathrm{~m}^{3}$, but has $19,668 \mathrm{~m}^{3}$ with no shipping information.

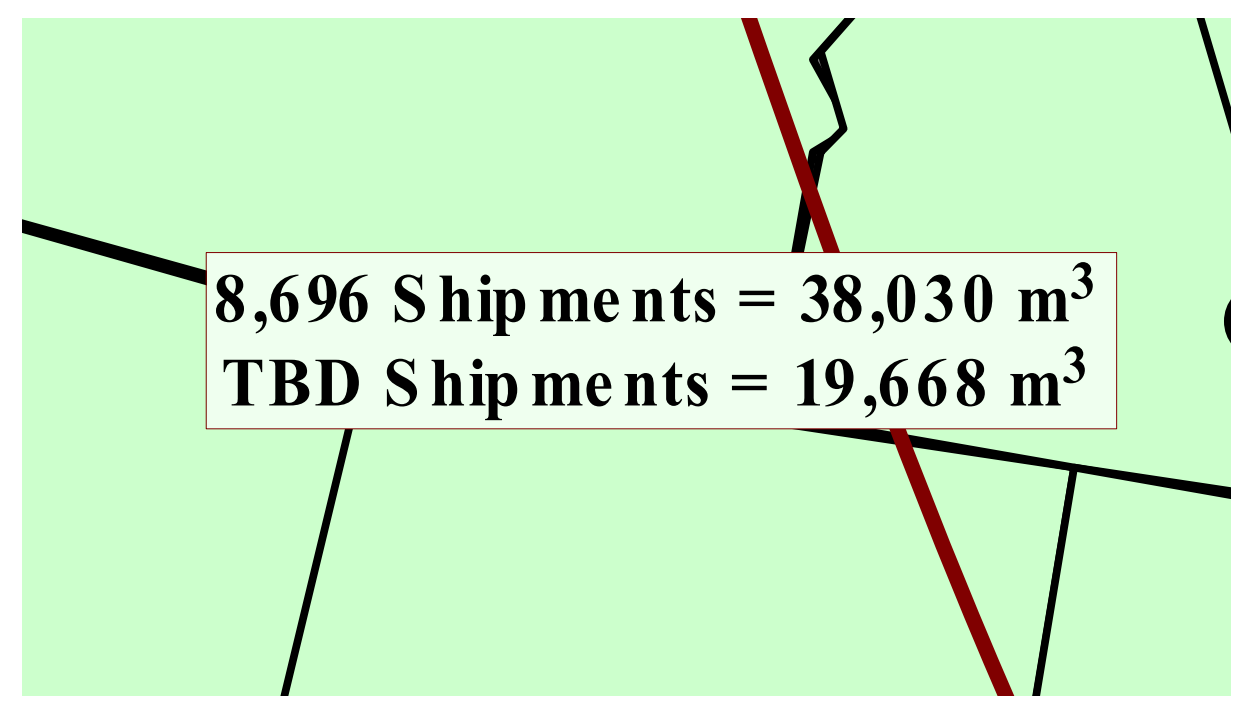

Figure 4. Volume reported in IPABS SDD with and without shipping information. 
Following the baseline schedule map are graphs showing the detailed information used to generate the map. The graphics include:

- A composite graph of the annual number of shipments by origin/destination used to obtain the total life-cycle number of shipments (for example, see Figure 5),

- A site specific graph of the annual number of shipments from/to the origin/destination (for example, see Figure 6),

- A composite graph of the origin/destination of the total life-cycle volume where shipping details were not available (for example, see Figure 7) and,

- A site specific graph of the annual volume from/to the origin/destination (for example, see Figure 8).

Details of the graphics are as follows:

The total life-cycle number of shipments to/from a given site is displayed in a composite graph. Figure 5 is an example of a composite graph showing incoming shipments to Hanford. It is a high level schedule of the incoming number of shipments by origin/destination.
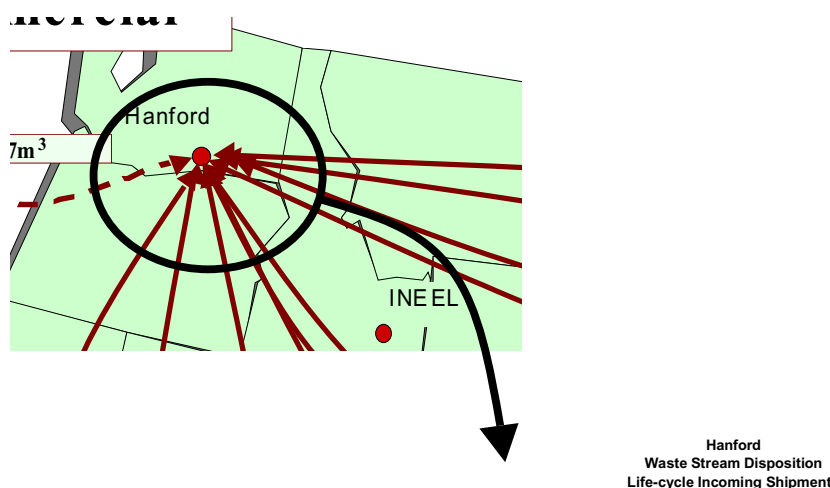

The composite graph showing incoming shipments to Hanford appears immediately following the Transportation Baseline Schedule map.

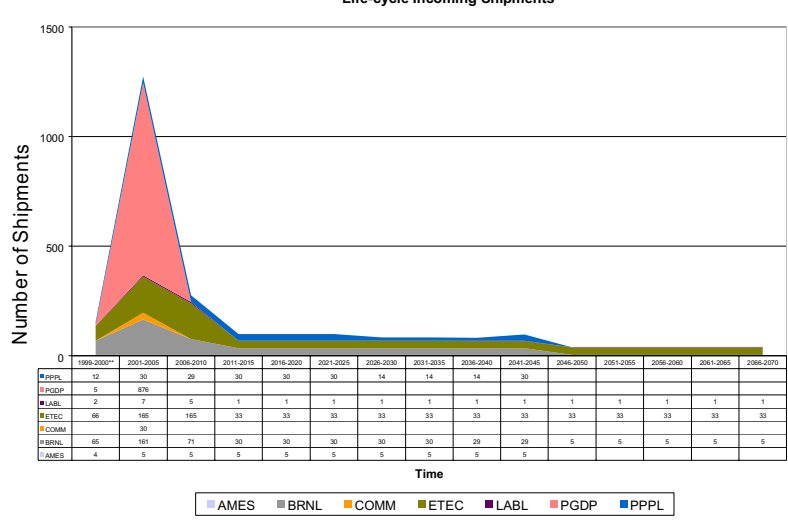

Figure 5. Composite graph showing incoming shipments to Hanford. 
The data used to ascertain the total life-cycle number of shipments for a given origin destination pair (as seen in the information box) is displayed in a graph. It shows the combined shipping schedule for all waste/material streams for a given site.

Figure 6 is an example of a site specific shipping schedule graph. It shows the annual number of shipments to the specified destination. A site specific shipping schedule graph for each of the sites shipping to Hanford is included after the composite graph. This graph will include all waste/material streams to be shipped from the origin to Hanford.

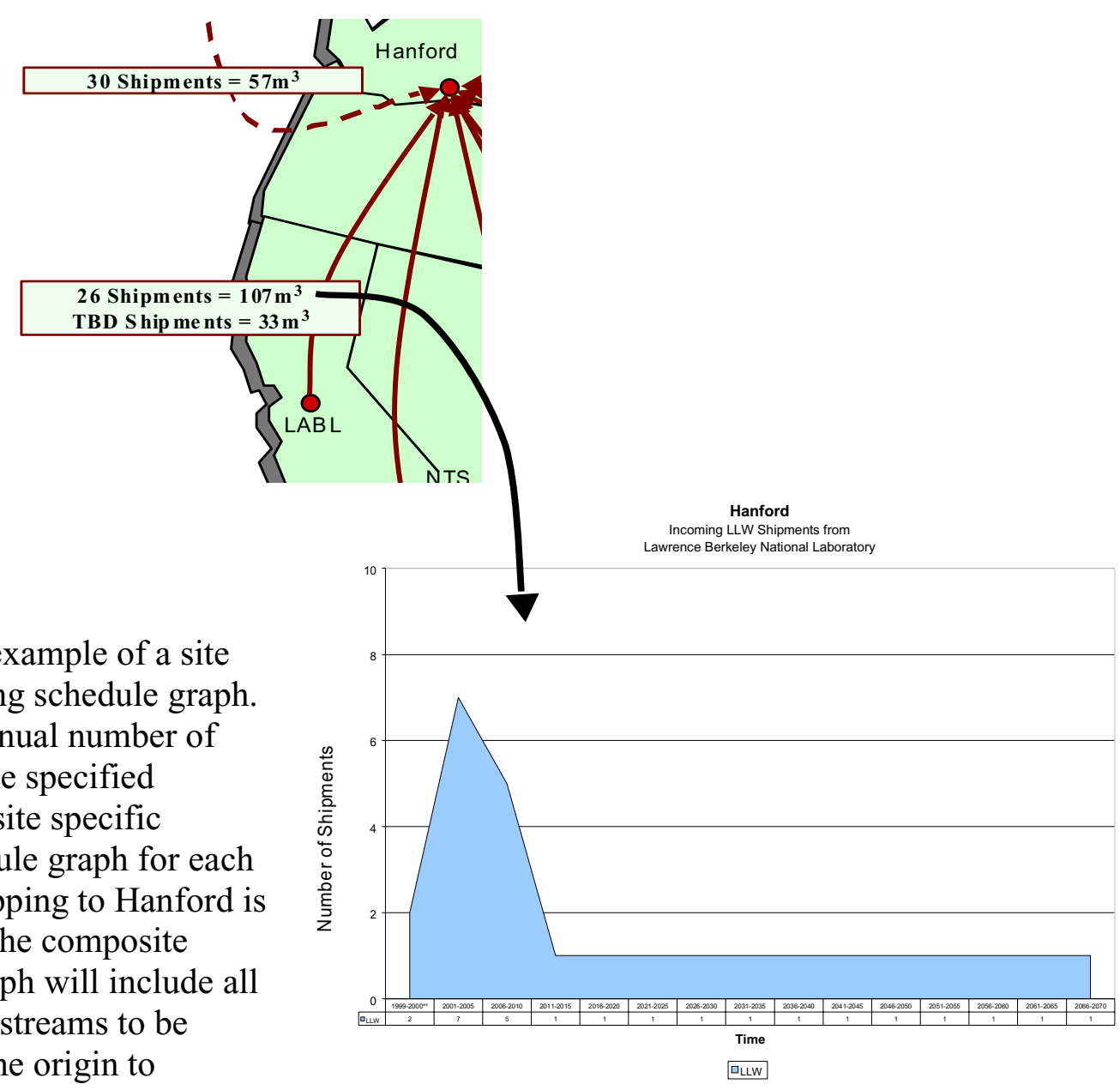

Figure 6. Site specific number of shipments schedule. 
For waste/material streams where no number of shipments was provided, a volume graph is included. As with the "number of shipments" graphs, a composite graph of the incoming volume is followed by graphs showing a site specific volume schedule. Figures 7 and 8 are examples of this situation.

Figure 7 is a composite graph of incoming volume. It includes only those incoming volumes that did not have associated shipping information. If present, the composite volume graph follows the site specific "number of shipments" graphs.

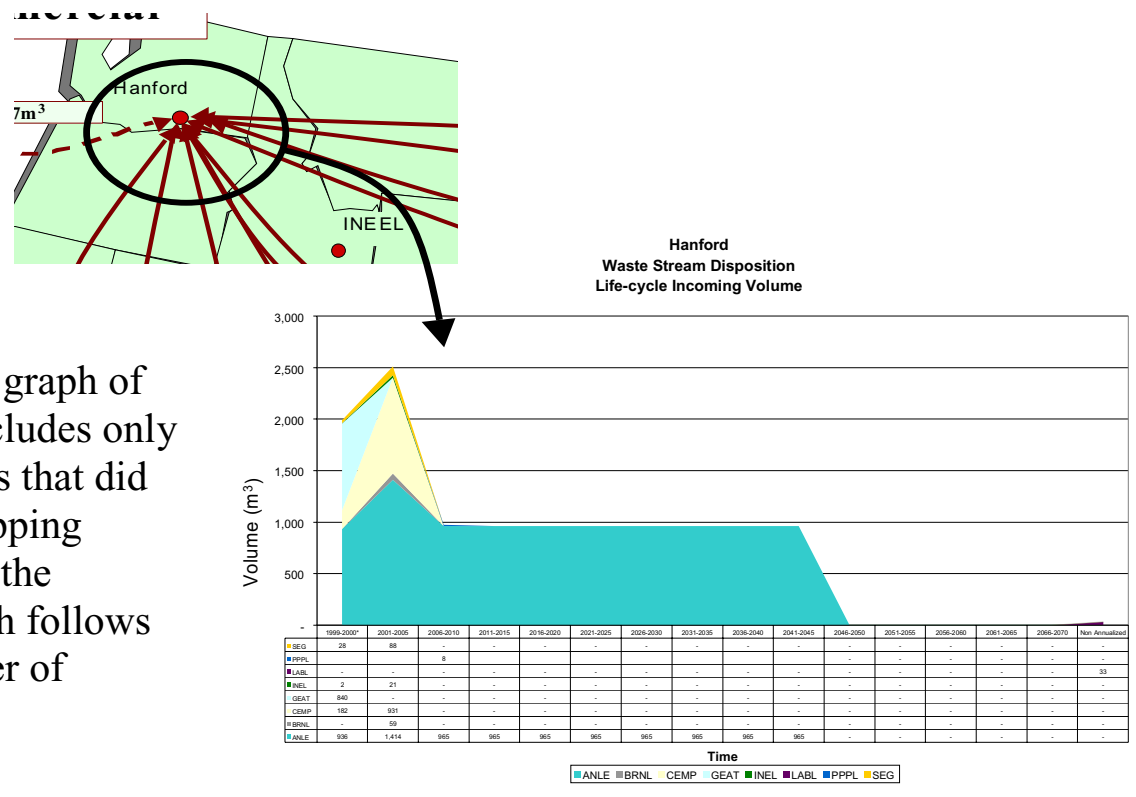

Figure 7. Composite graph showing incoming volume to Hanford.

If "TBD Shipments $=\mathrm{x}$ m3" appears in the information box, a schedule graph of volume to be shipped is provided. Figure 8 is an example of a site specific volume schedule. If needed, it is located after the composite volume graph. 
The volume graphs show metric tons of heavy metal (MTHM), metric tons (MT), or number of canisters (NC) as needed.

Two maps were developed for Nevada Test Site (NTS). One map shows the incoming shipments recorded in the SDD. The second map includes the SDD shipments to NTS and all shipments of LLW to "TBD" and "TBDO." They were included as "potential" shipments to NTS.

\subsection{Summary}

The "Number of Shipments" data element captured in IPABS was used to generate a baseline shipping schedule. Annual numbers of shipments were combined to obtain the total life-cycle number of shipments for a given site. That information is graphically displayed in an integrated view of the transportation activities for a given site. A graph compiling all shipments to/from a site by origin/destination was developed. The combination of these pieces of information provides a graphical view of the shipping schedule to and from each of the 11 major sites and the Geologic Repository Disposal site. 


\section{ABBREVIATIONS and GEOGRAPHICAL SITE ACRONYM LIST}

\begin{tabular}{|c|c|}
\hline AEMP & Ashtabula Environmental Management Project \\
\hline AMES & Ames Laboratory \\
\hline ANLE & Argonne National Laboratory - East \\
\hline ANLW & Argonne National Laboratory - West \\
\hline $\mathrm{ARCO}$ & ARCO Medical Products Company \\
\hline ARMY & U.S. Army Material Command \\
\hline BAPL & Bettis Atomic Power Laboratory \\
\hline BRNL & Brookhaven National Laboratory \\
\hline CEMP (BCLJ) & Columbus Environmental Management Project - West Jefferson \\
\hline CNS & Charleston Naval Shipyard \\
\hline COMM & Commercial Site - TBD \\
\hline CWM & Chemical Waste Management \\
\hline DOE & DOE Site - TBD \\
\hline DSSI & Diversified Scientific Services, Inc. \\
\hline ENVR & Envirocare \\
\hline ETEC & Energy Technology Engineering Center \\
\hline FEMP & Fernald Environmental Management Project \\
\hline GEAT & General Atomics \\
\hline GRD & Geologic Repository Disposal \\
\hline HASI & Hanford Site \\
\hline HLW & High Level Waste \\
\hline INEL, INEEL & Idaho National Engineering and Environmental Laboratory \\
\hline KAPL & Knolls Atomic Power Laboratory - Schenectady \\
\hline KESS & Knolls Atomic Power Laboratory - Kesselring \\
\hline KWIN & Knolls Atomic Power Laboratory - Windsor \\
\hline LABL & Lawrence Berkeley National Laboratory \\
\hline LANL & Los Alamos National Laboratory \\
\hline LL & Laidlaw \\
\hline LLMS & Lawrence Livermore National Laboratory - Main Site \\
\hline LLW & Low Level Waste \\
\hline LRRI & Lovelace Respiratory Research Institute \\
\hline M/LLW & Mixed Low Level Waste and Low Level Waste \\
\hline MEMP & Miamisburg Environmental Management Project \\
\hline MINS & Mare Island Naval Shipyard \\
\hline MLLW & Mixed Low Level Waste \\
\hline MURR & Missouri University Research Reactor \\
\hline NAVY & Naval Reactors Site - TBD \\
\hline NNS & Norfolk Naval Shipyard \\
\hline NRF & Naval Reactor Facility \\
\hline NVTS, NTS & Nevada Test Site \\
\hline ORR, ORTN & Oak Ridge Reservation \\
\hline PAPL & Pantex Plant \\
\hline PGDP & Paducah Gaseous Diffusion Plant \\
\hline PHNS & Pearl Harbor Naval Shipyard \\
\hline PNS & Portsmouth Naval Shipyard \\
\hline
\end{tabular}




\section{ABBREVIATIONS and GEOGRAPHICAL SITE ACRONYM LIST}

\begin{tabular}{|l|l|}
\hline PORT & Portsmouth Gaseous Diffusion Plant \\
\hline PPPL & Princeton Plasma Physics Laboratory \\
\hline PSNS & Puget Sound Naval Shipyard \\
\hline RFTS & Rocky Flats Environmental Technology Site \\
\hline SARS, SRS & Savannah River Site \\
\hline SEG & GTS Duratek (formerly, Scientific Ecology Group) \\
\hline SK & SafetyKleen \\
\hline SNF & Spent Nuclear Fuel \\
\hline SNLN & Sandia National Laboratories - NM \\
\hline SPRU & Separations Process Research Unit \\
\hline TBD & To Be Determined \\
\hline TBDO & To Be Determined/Off-Site \\
\hline TRU & Transuranic Waste \\
\hline WIPP & Waste Isolation Pilot Plant \\
\hline WVDP & West Valley Demonstration Project \\
\hline
\end{tabular}




\section{GLOSSARY}

Hazardous Material

High Level Radioactive Waste

Low Level Radioactive Waste

Mixed Waste

Spent Nuclear Fuel

Transuranic Waste
A substance or material, which has been determined by the Secretary of Transportation to be capable of posing an unreasonable risk to health, safety, and property when transported in commerce, and which has been so designated. The term includes hazardous substances, hazardous wastes, marine pollutants, and elevated temperature materials as defined in this section, materials designated as hazardous under the provisions of Sec. 172.101 of this subchapter, and materials that meet the defining criteria for hazard classes and divisions in Part 173 of this subchapter (49 CFR 171.8).

(A) The highly radioactive material resulting from the reprocessing of spent nuclear fuel, including liquid waste produced directly in reprocessing and any solid material derived from such liquid waste that contains fission products in sufficient concentrations; and (B) other highly radioactive material that the Commission consistent with existing law, determines by rule requires permanent isolation (42 USC 10101, 10 CFR 72.3, 10 CFR 960.2).

Radioactive material that (A) is not high-level radioactive waste, spent nuclear fuel, Transuranic waste, or by-product material as defined in section 2014(e)(2) of this title; and (B) the NRC Commission, consistent with existing law, classifies as low level radioactive waste (42 USC 10101, 10 CFR 62.2).

Waste that contains both hazardous waste and source special nuclear, or by-product material subject to the Atomic Energy Act of 1954 (42 U.S.C. 2011 et seq., 42 USC 6903).

Fuel that has been withdrawn from a nuclear reactor following irradiation, the constituent elements of which have not been separated by reprocessing (42 USC 10101, 10 CFR 960.2).

Material contaminated with elements that have an atomic number greater than 92, including neptunium, plutonium, americium, and curium, and that are in concentrations greater than $10 \mathrm{nCi} / \mathrm{g}$, or in such other concentrations as the Nuclear Regulatory Commission may prescribe to protect the public health and safety (42 USC 2014). 



\section{Attachment A}

\section{Transportation Baseline Schedule Maps and \\ Number of Shipments/Volume Graphs}





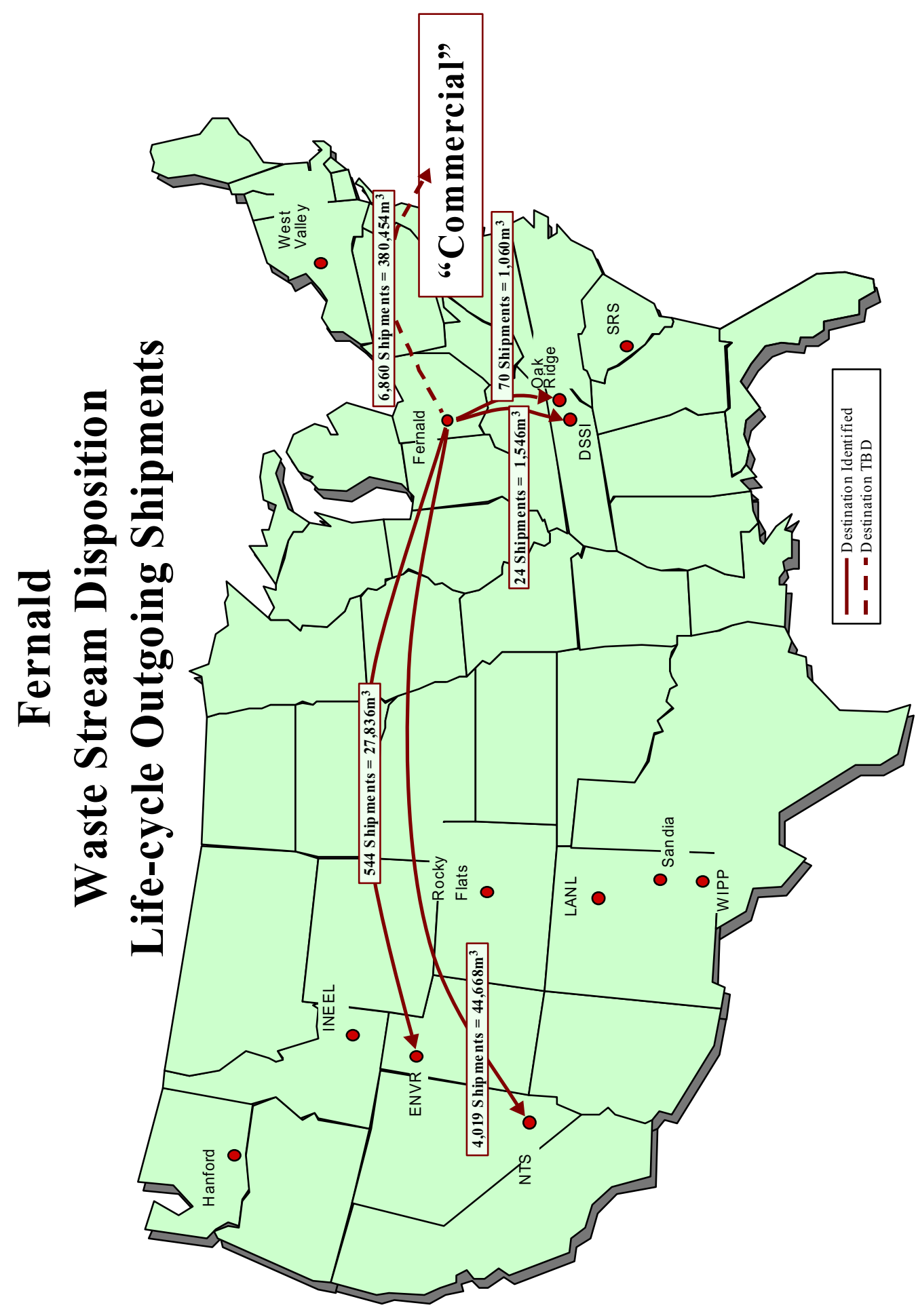




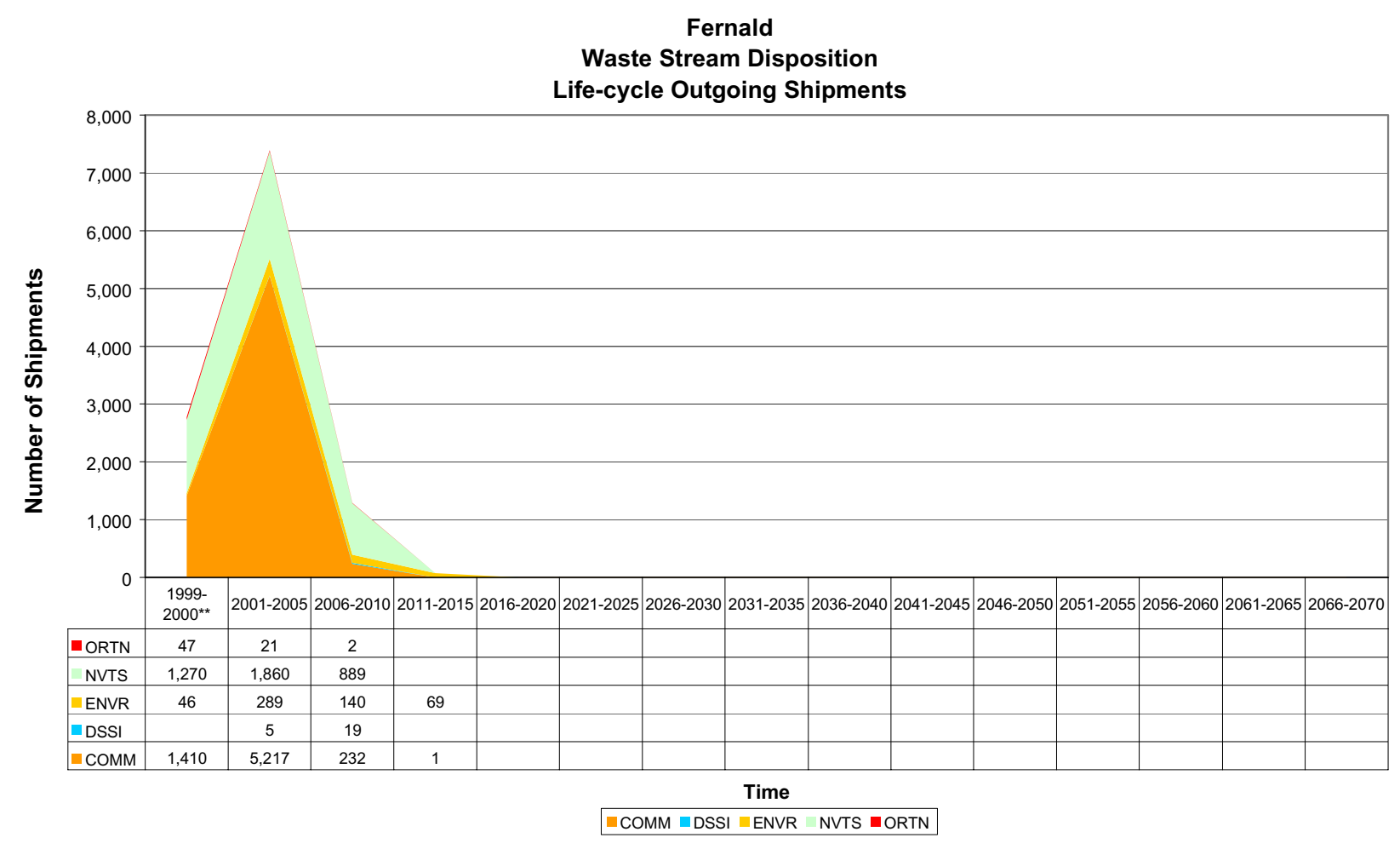

Fernald

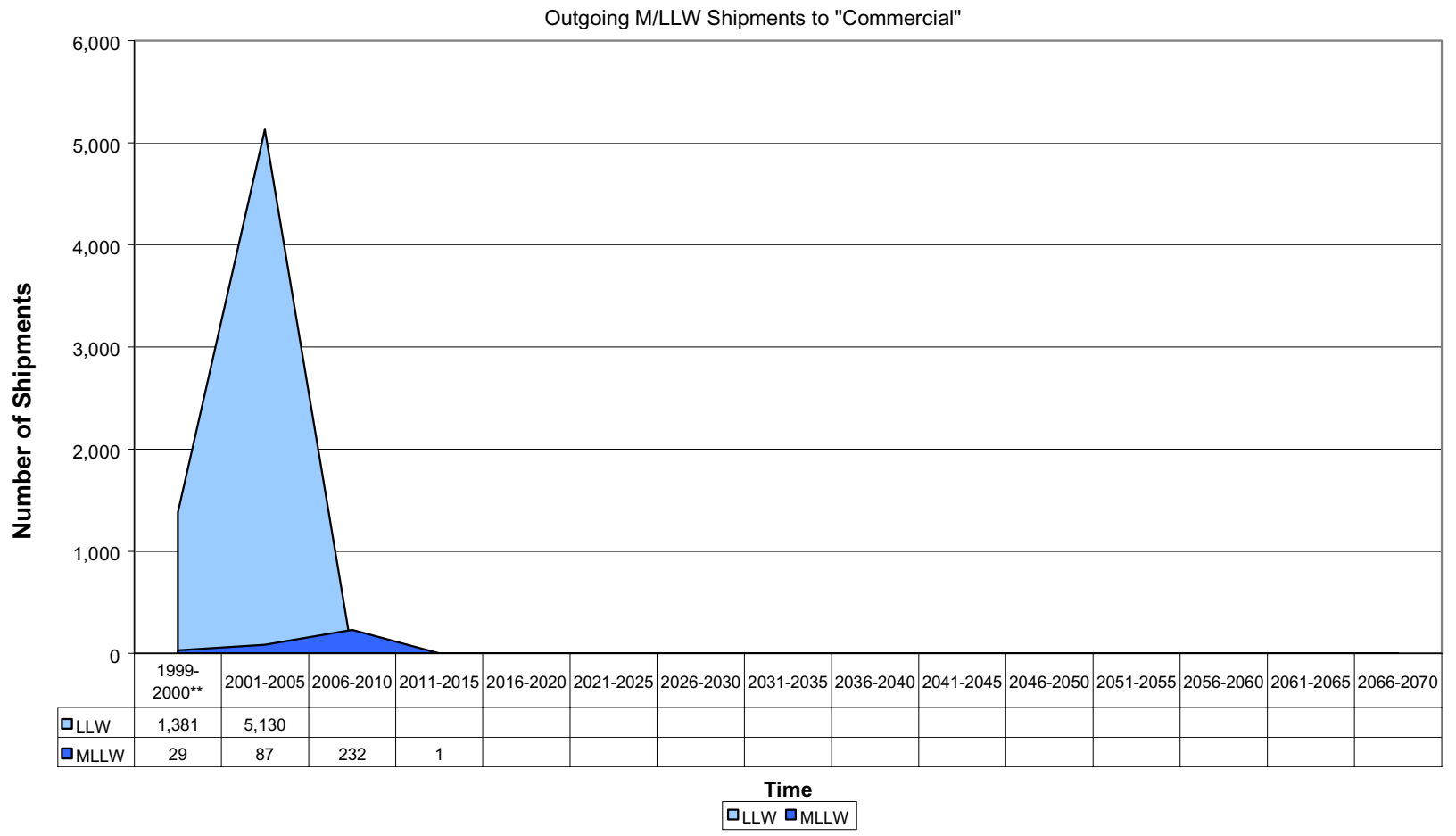

Total life-cycle values per 7/30/99 Stream Disposition Data (SDD) (http://id.inel.gov/avs/).

A-2

Printed: March 30, 2000

** Represents data for a two year period. 
Fernald

Outgoing MLLW Shipments to Diversified Scientific Services, Inc.

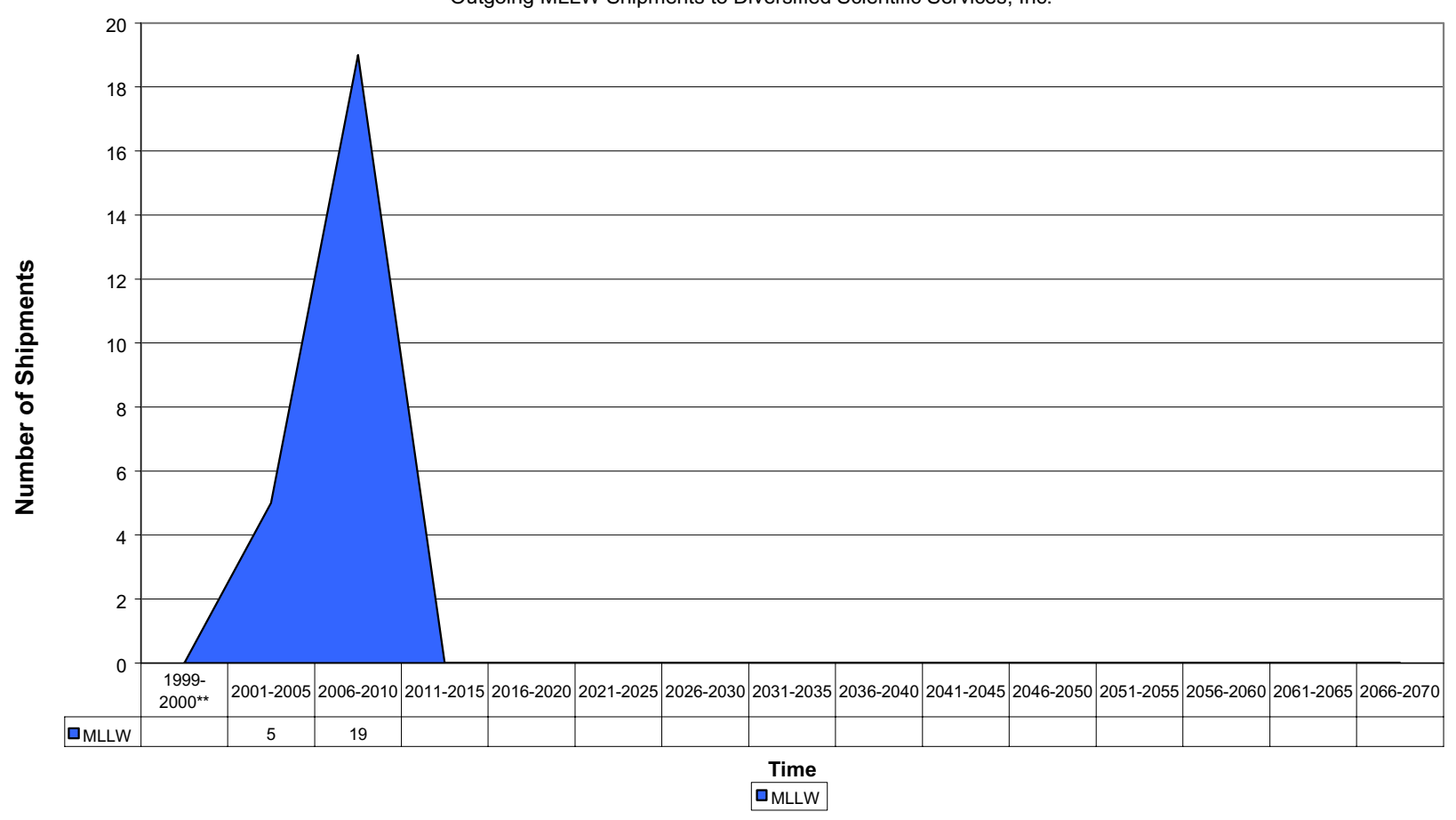

Fernald

Outgoing M/LLW Shipments to Envirocare

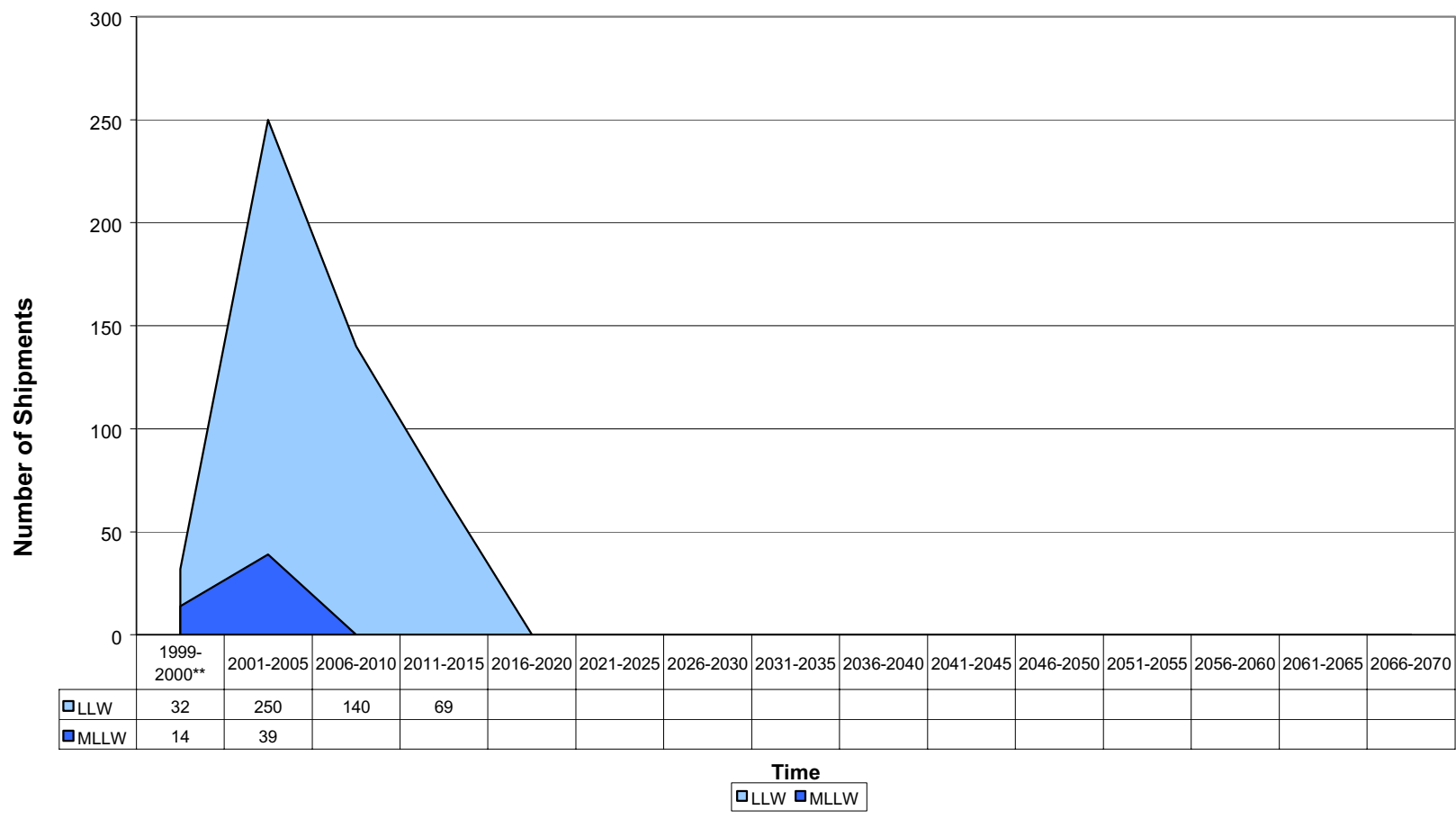

Total life-cycle values per 7/30/99 Stream Disposition Data (SDD) (http://id.inel.gov/avs/).

A-3

Printed: March 30, 2000

** Represents data for a two year period. 
Fernald

Outgoing LLW Shipments to Nevada Test Site

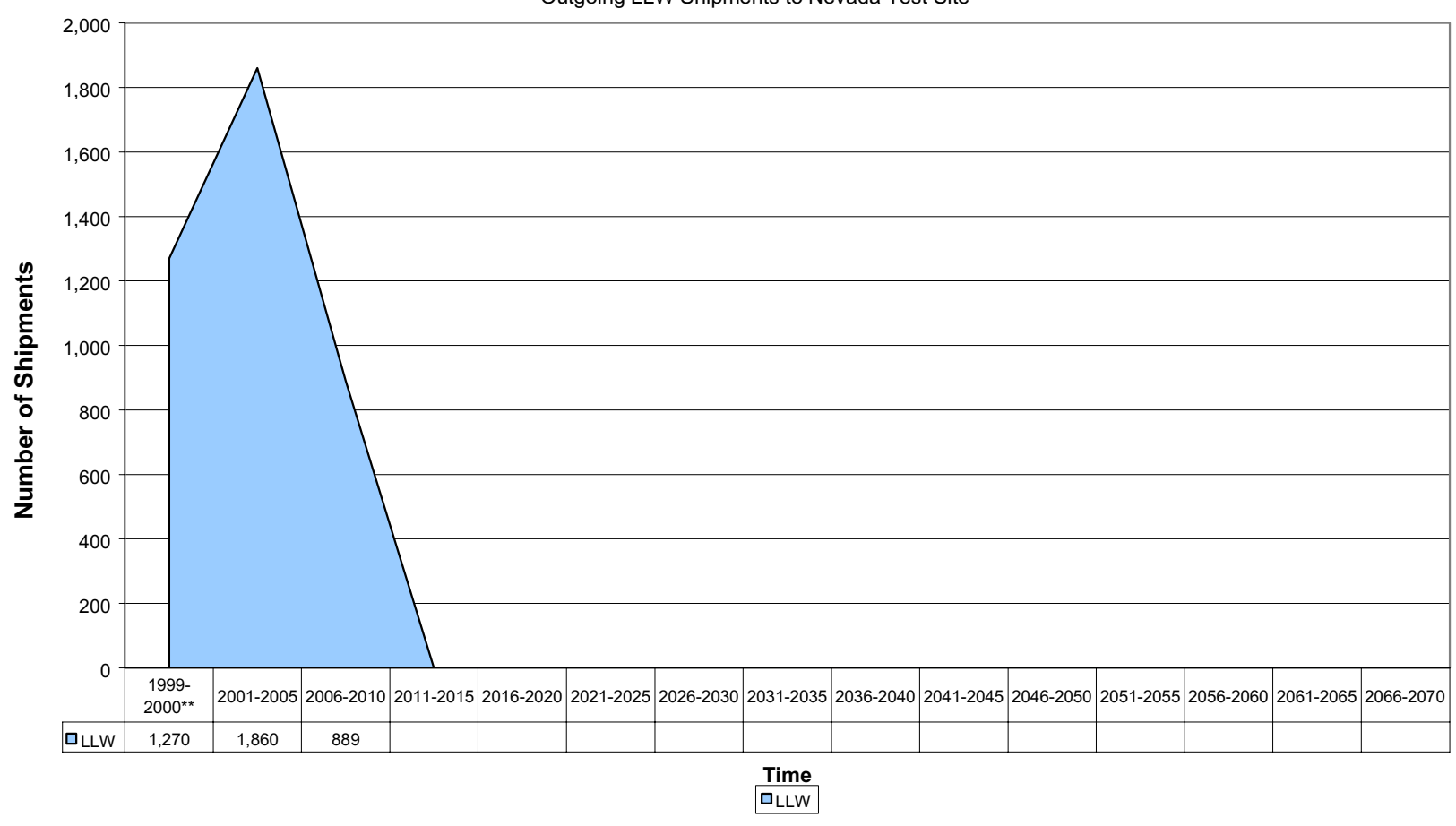

Fernald

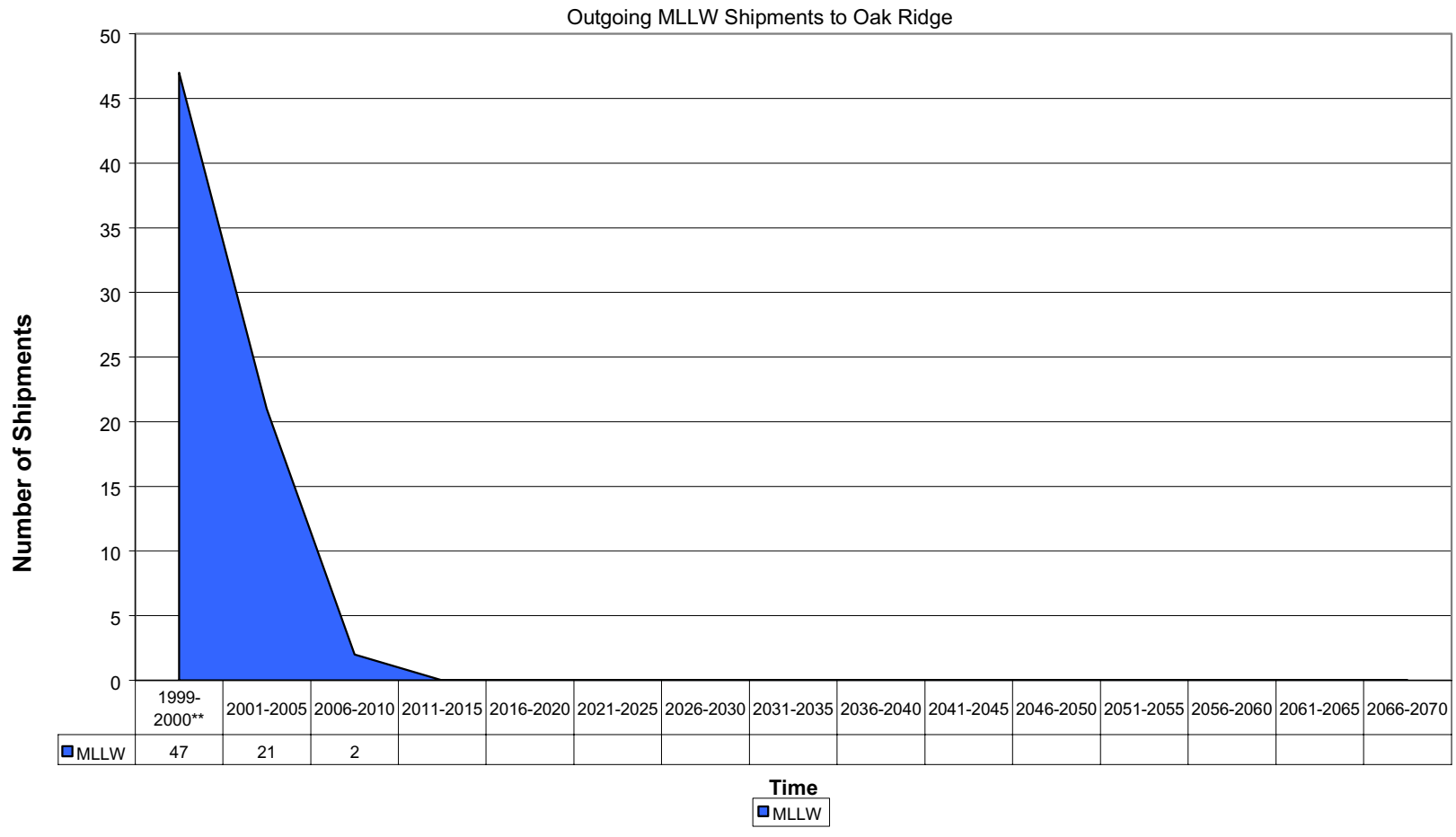

Total life-cycle values per 7/30/99 Stream Disposition Data (SDD) (http://id.inel.gov/avs/).

** Represents data for a two year period. 


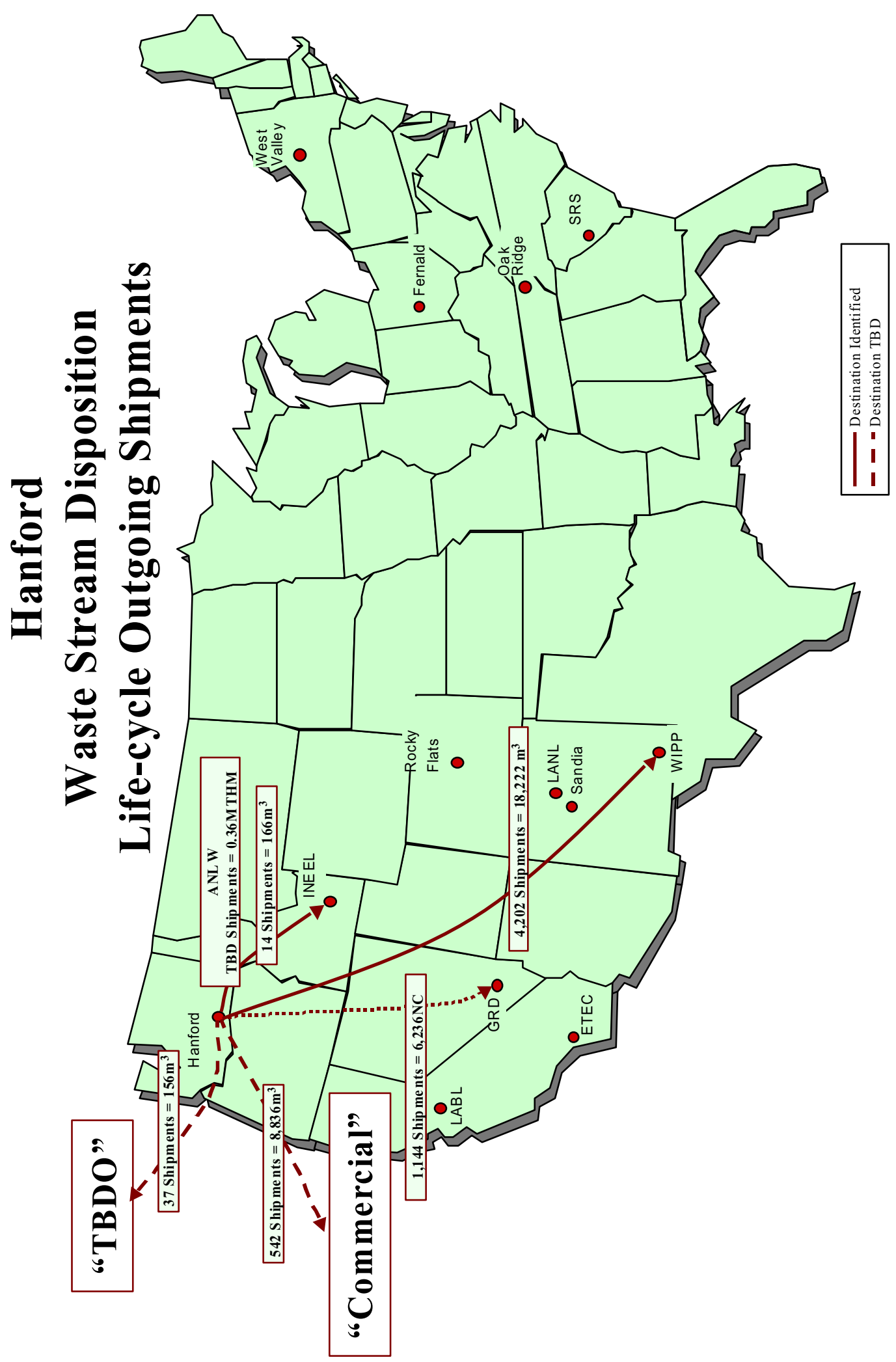




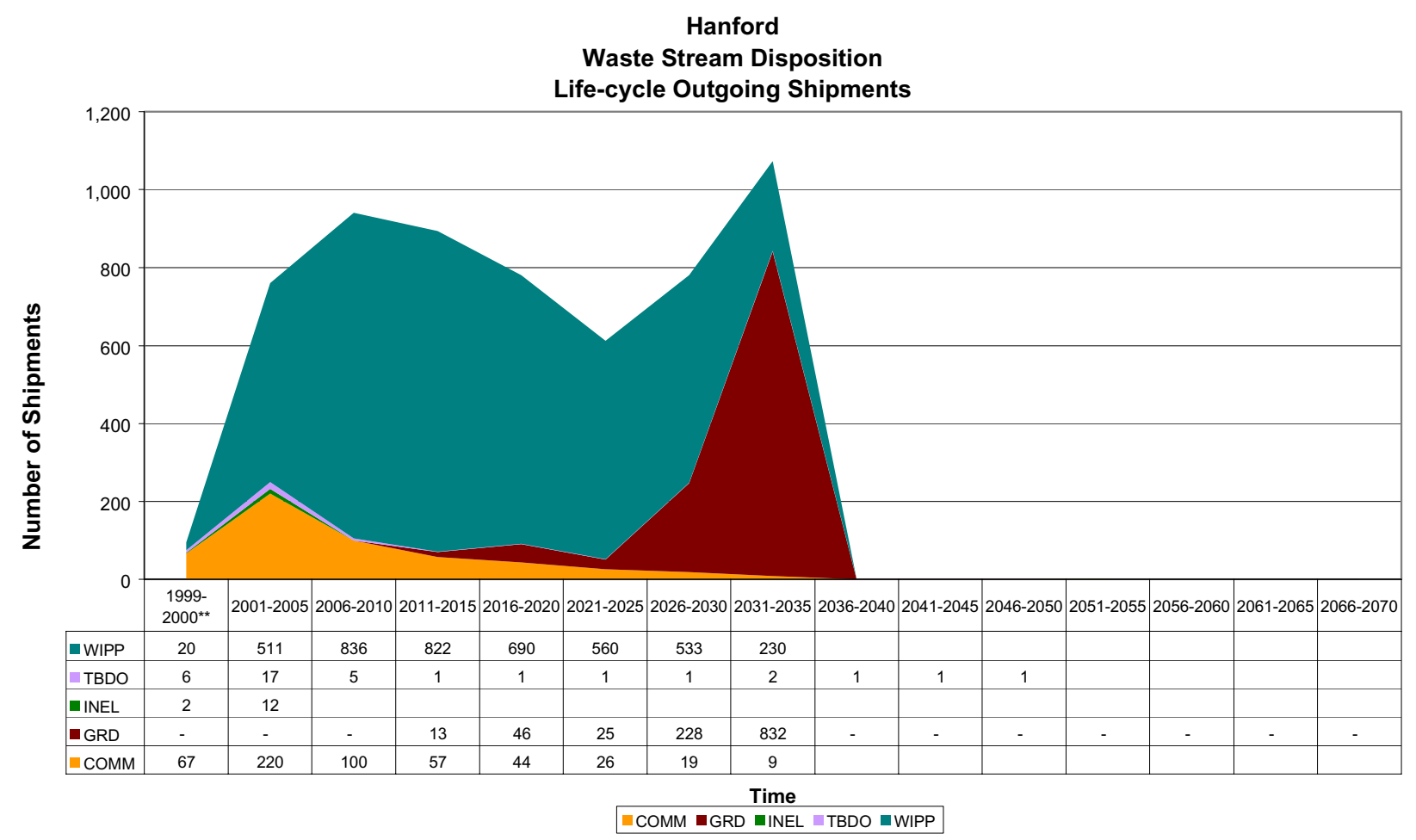

Hanford

Outgoing M/LLW Shipments to "Commercial"

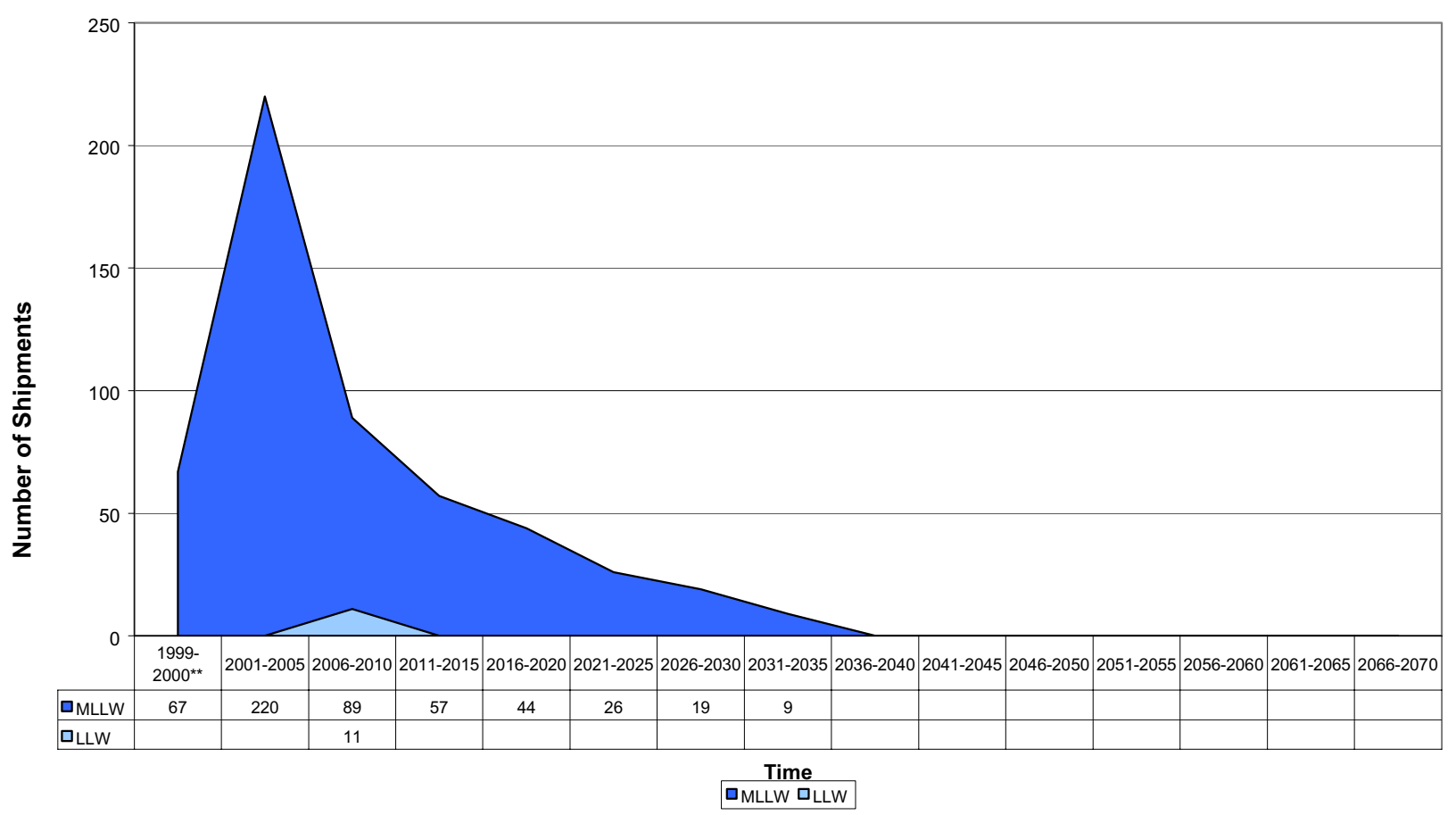

Total life-cycle values per 7/30/99 Stream Disposition Data (SDD) (http://id.inel.gov/avs/) 


\section{Hanford}

Outgoing HLW \& SNF Shipments to Geologic Repository Disposal

(Tentatively Yucca Mtn.)

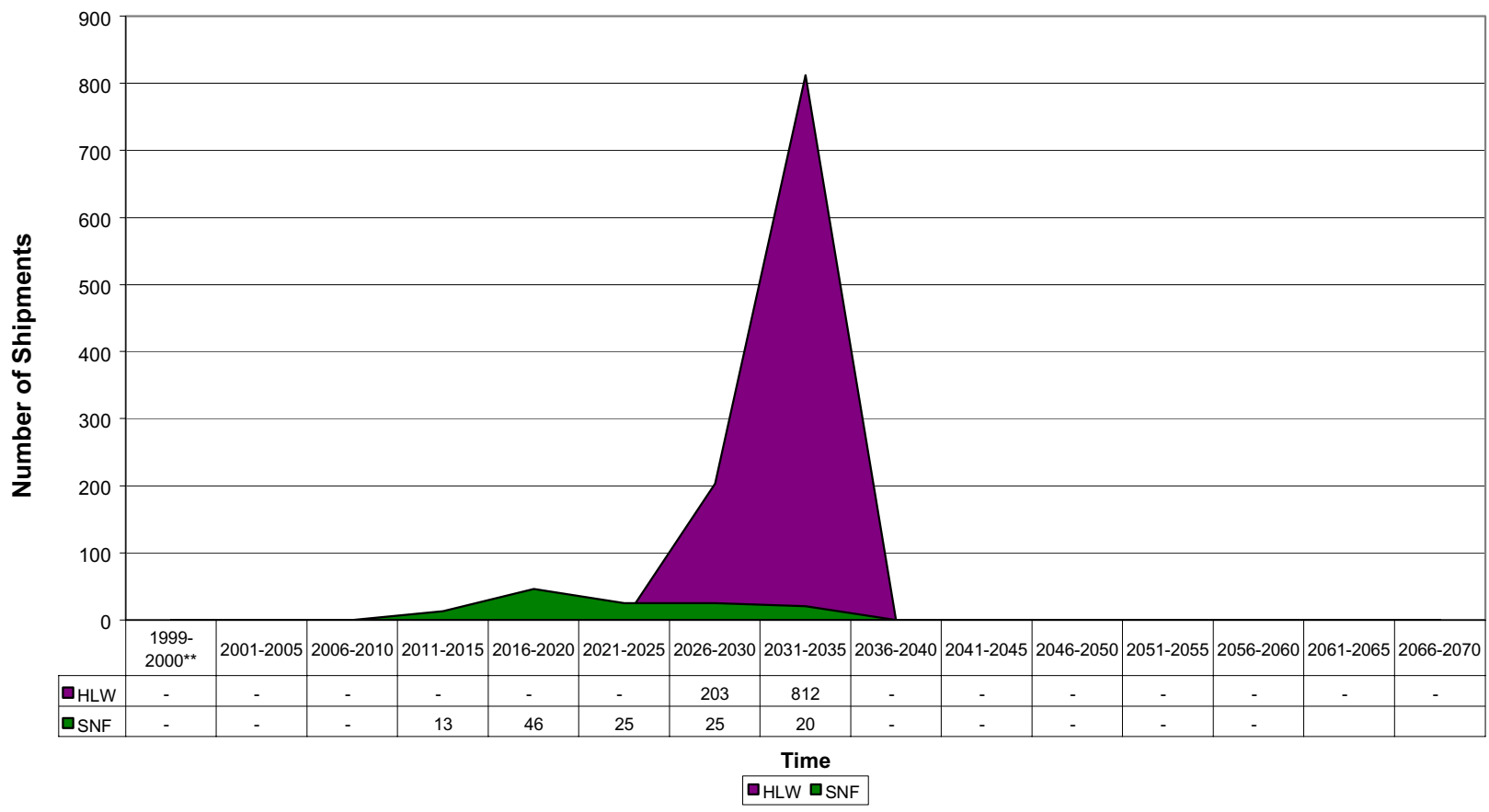

Hanford

Outgoing MLLW Shipments to INEEL

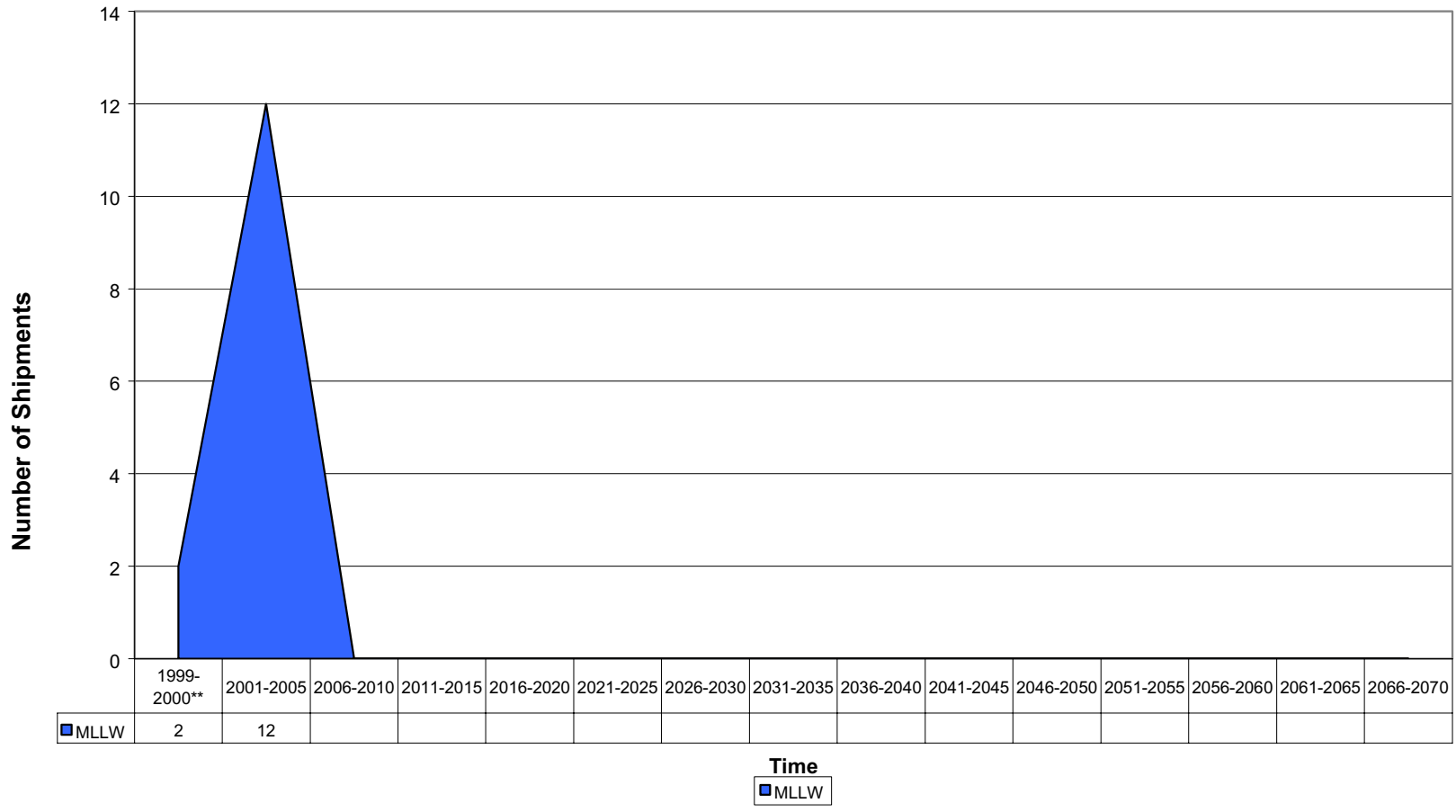

Total life-cycle values per 7/30/99 Stream Disposition Data (SDD) (http://id.inel.gov/avs/) 
Hanford

Outgoing MLLW Shipments to "TBDO"

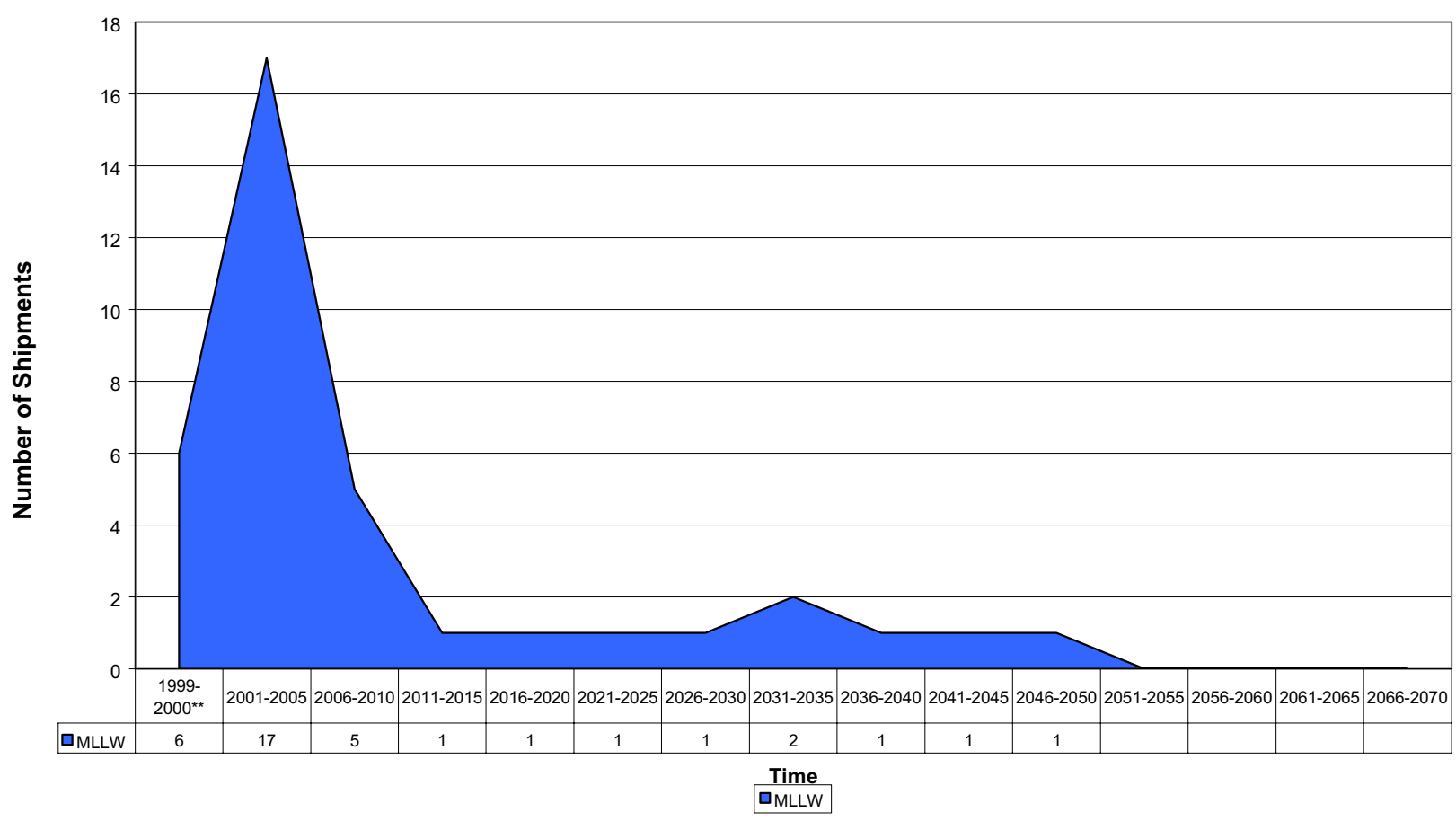

Hanford

Outgoing TRU Shipments to WIPP

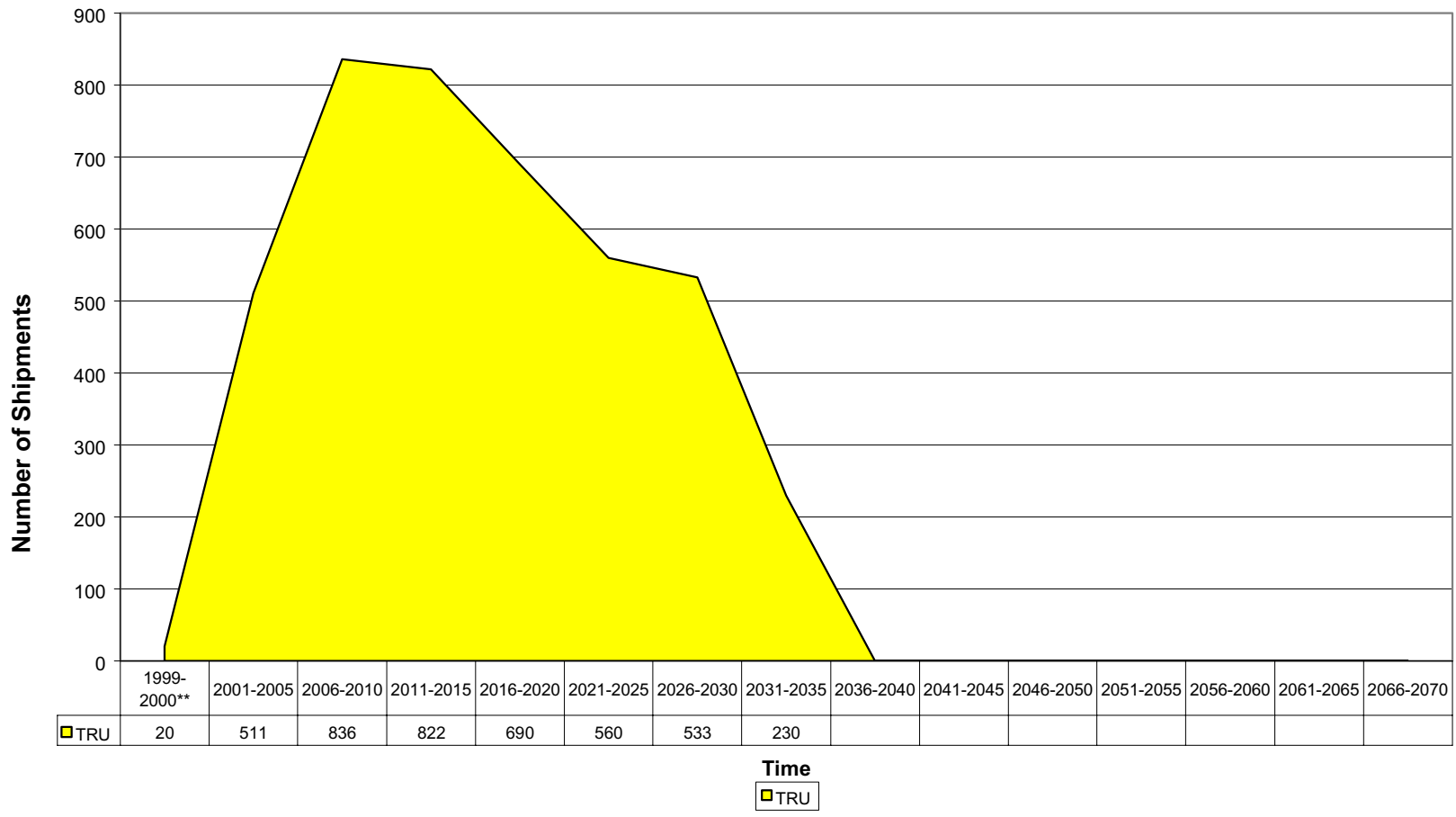

Total life-cycle values per 7/30/99 Stream Disposition Data (SDD) (http://id.inel.gov/avs/) 


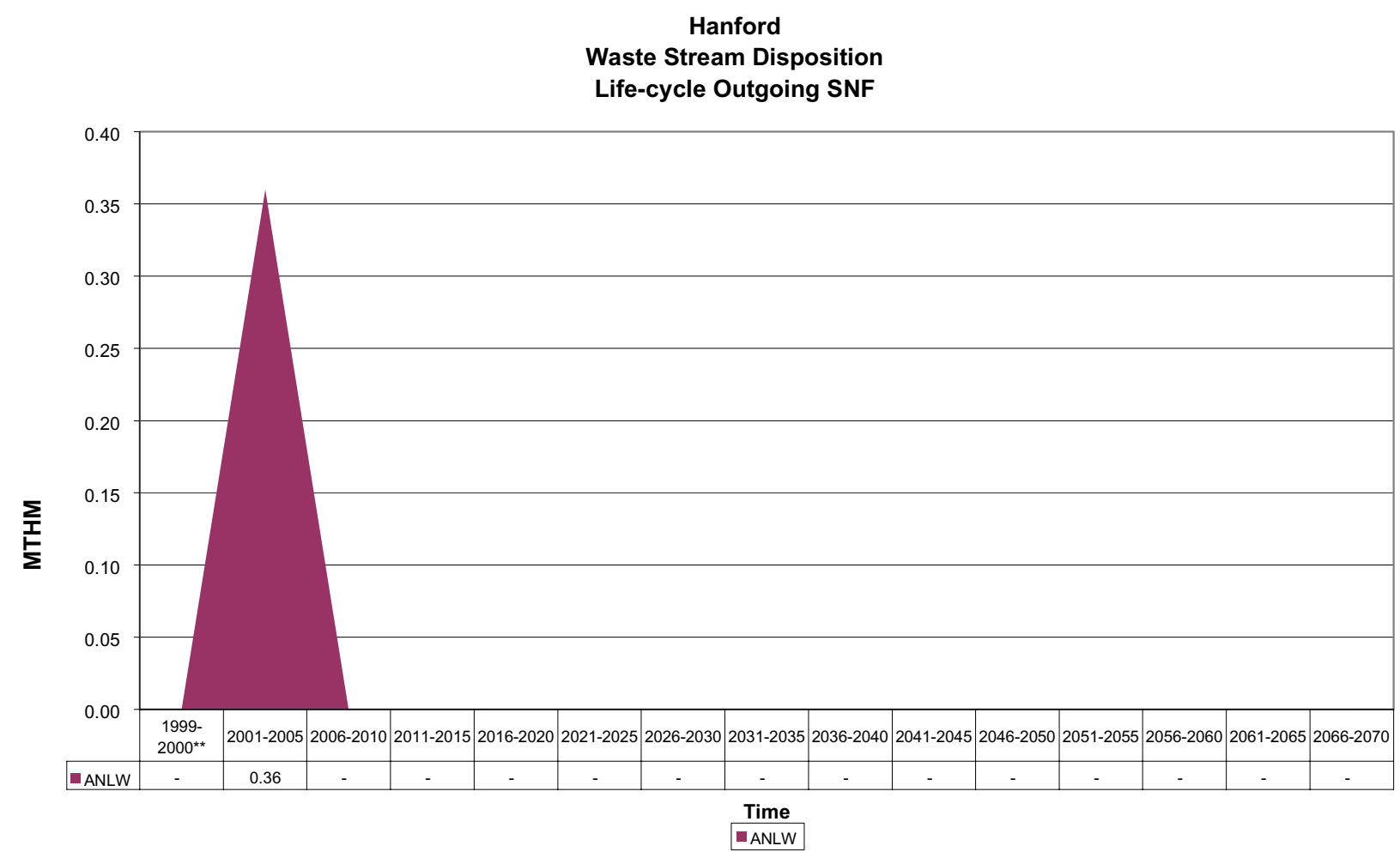

Hanford

Outgoing SNF to Argonne National Laboratory West

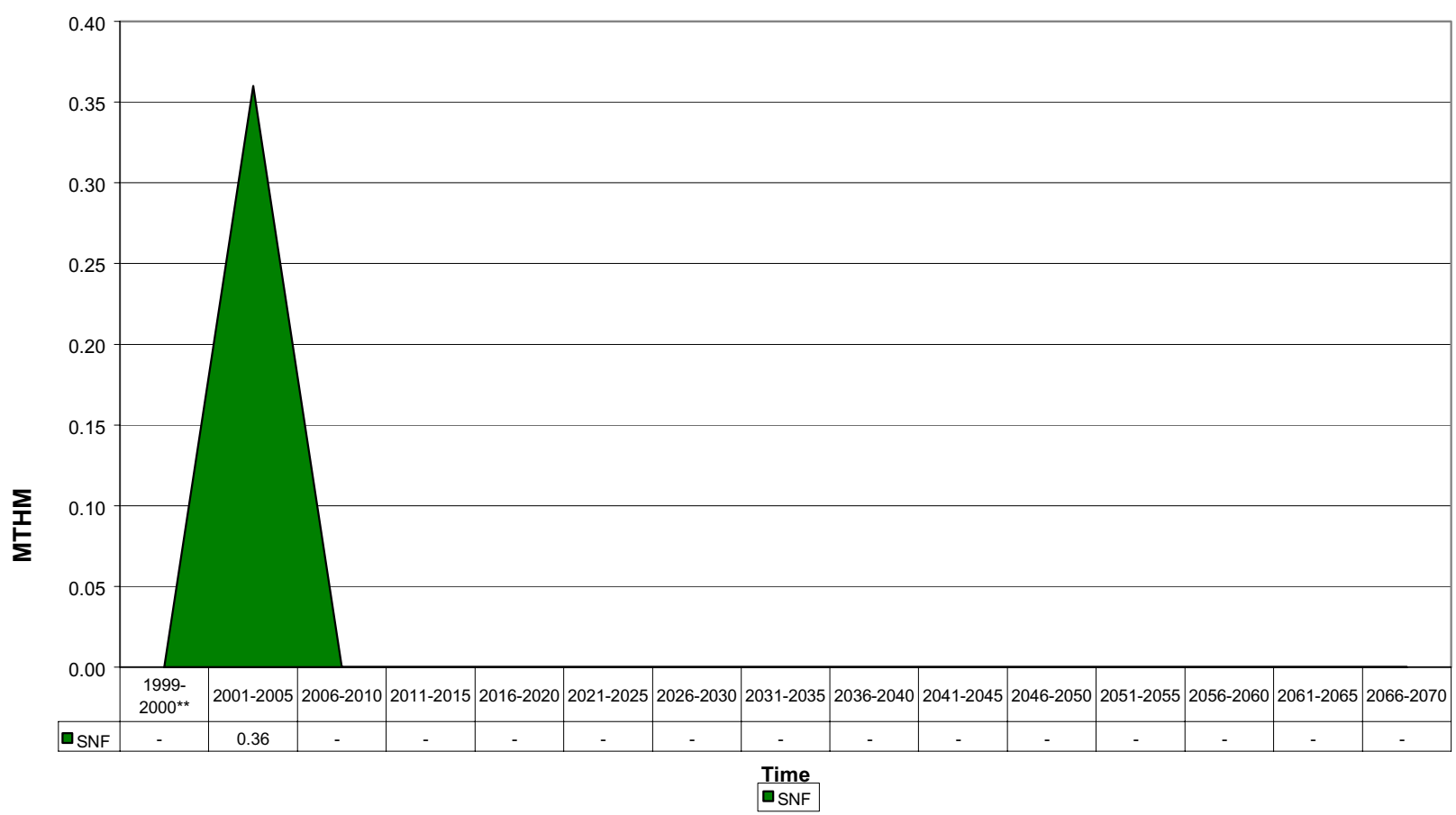

Total life-cycle values per 7/30/99 Stream Disposition Data (SDD) (http://id.inel.gov/avs/) 



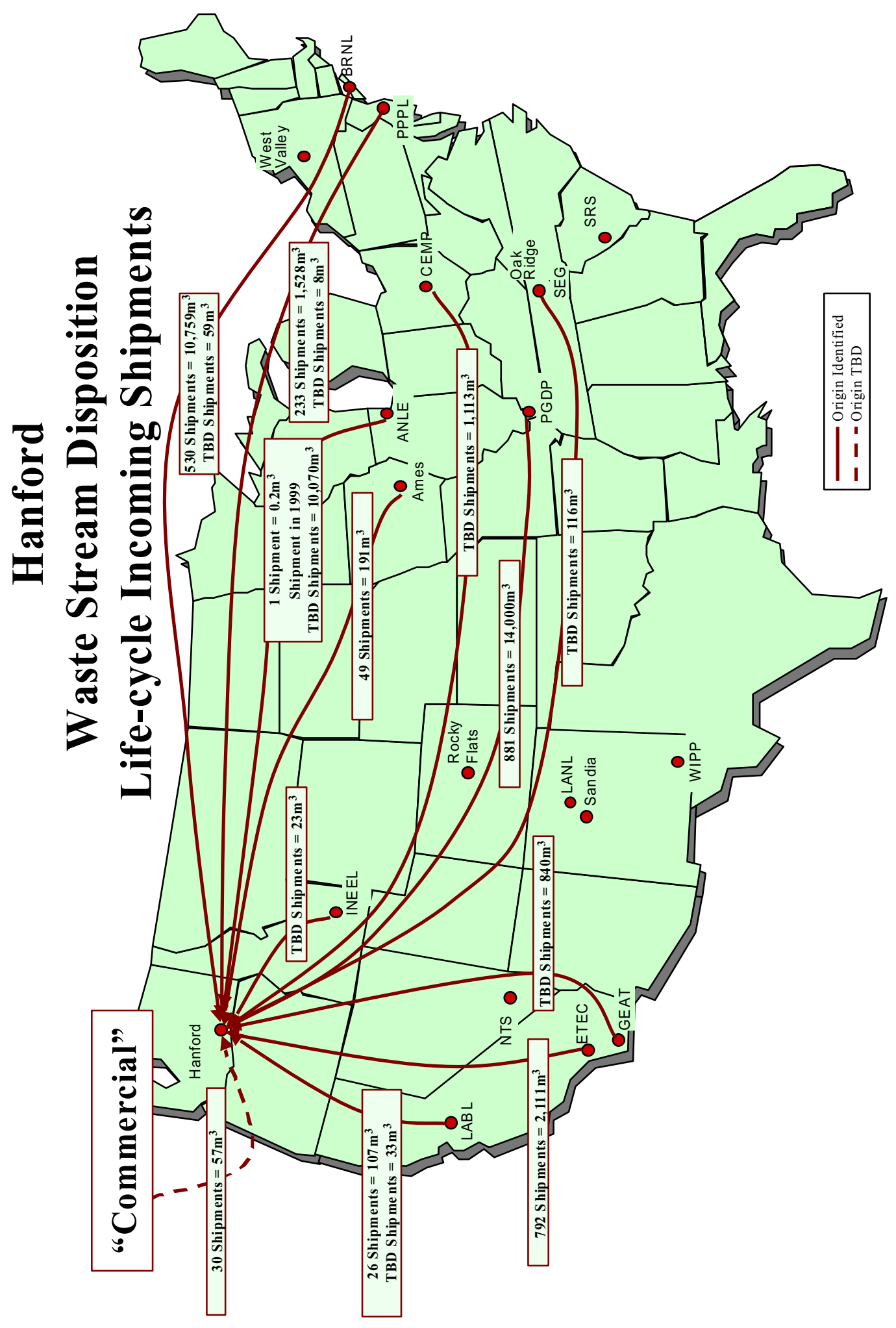


Hanford

Waste Stream Disposition

Life-cycle Incoming Shipments

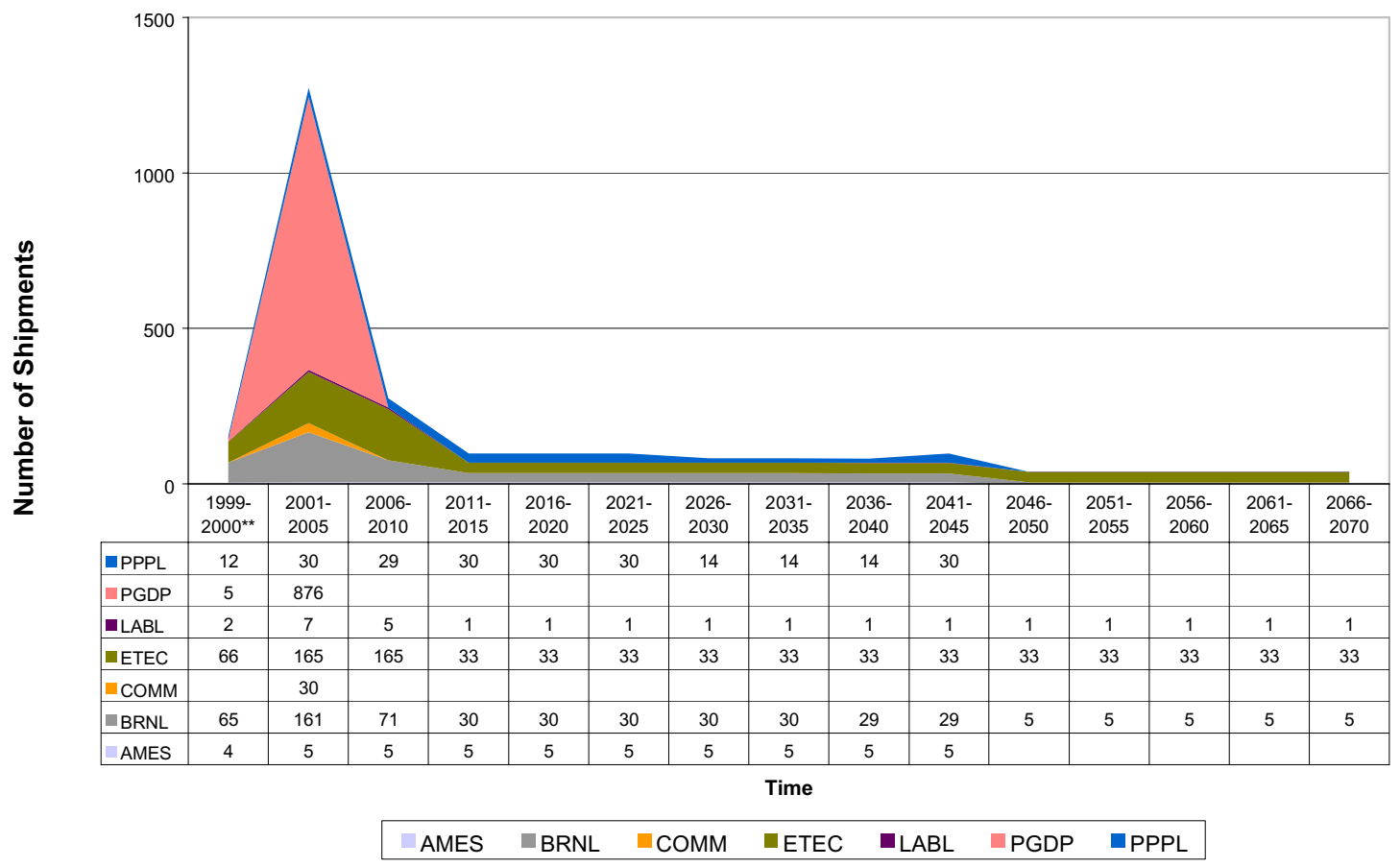

Hanford

Incoming LLW Shipments from "Commercial"

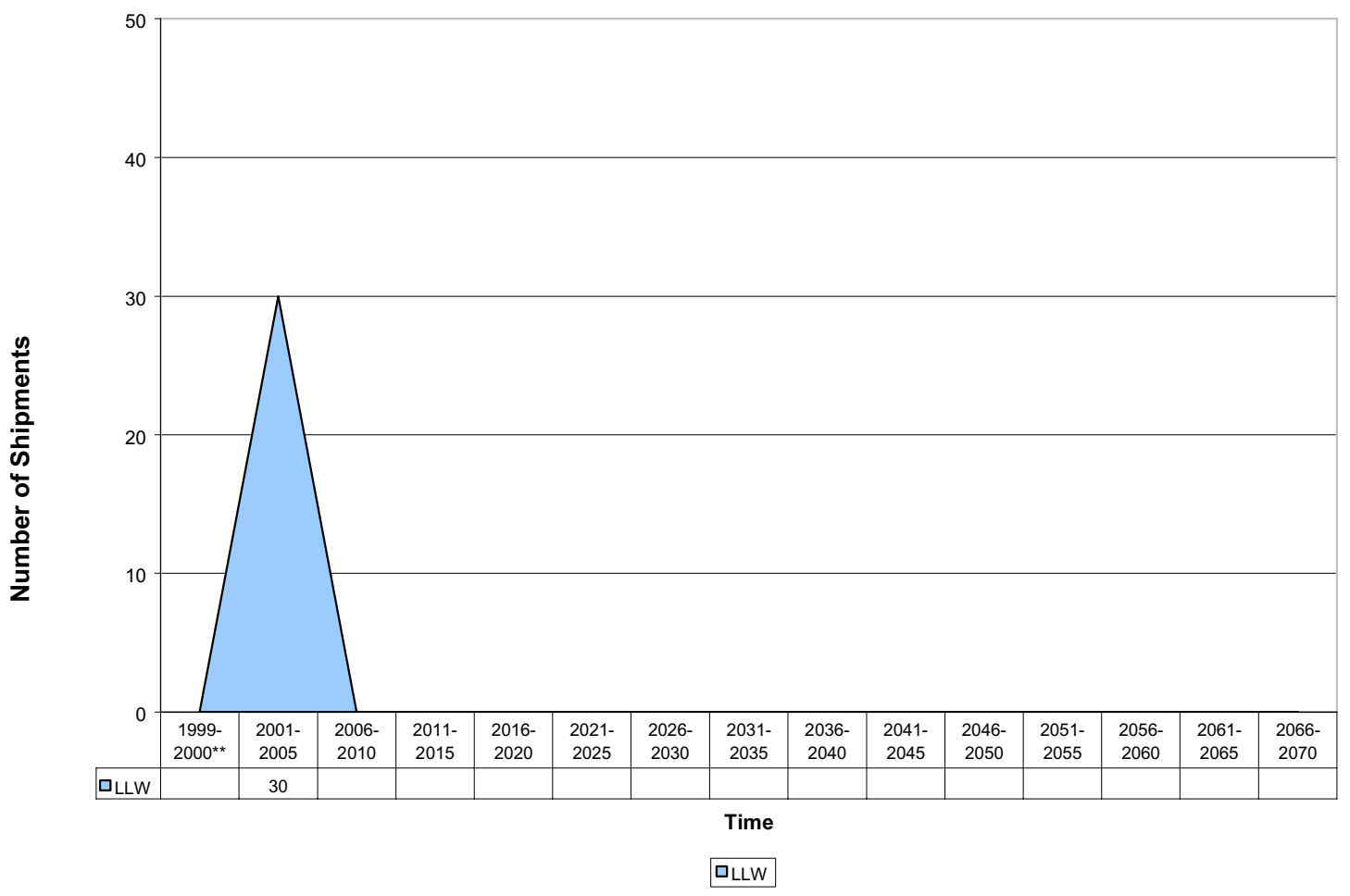

Total life-cycle values per 7/30/99 Stream Disposition Data (SDD) (http://id.inel.gov/avs/).

** Represents data for a two year period. 


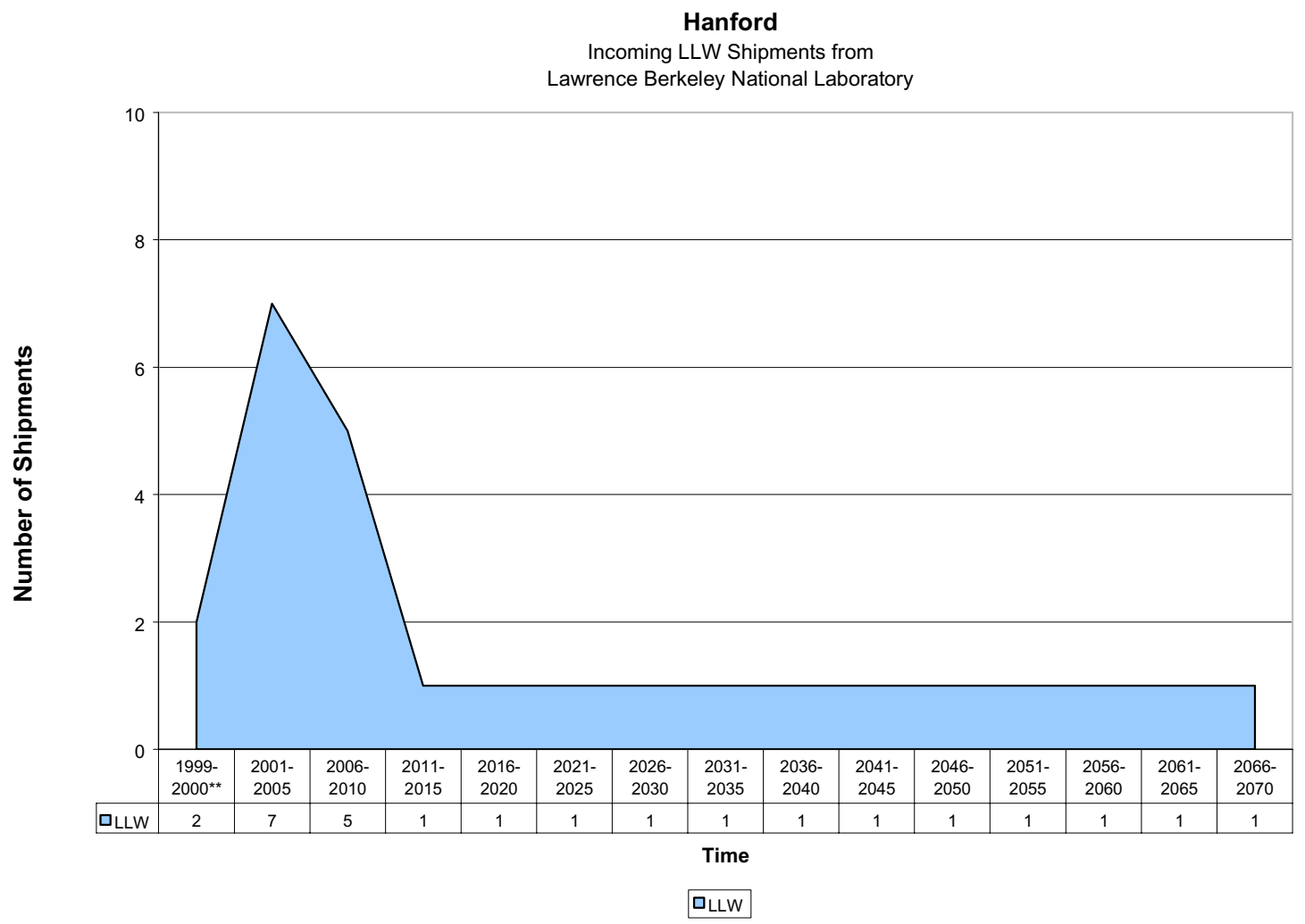

Hanford

Incoming LLW Shipments from

Energy Technology Engineering Center

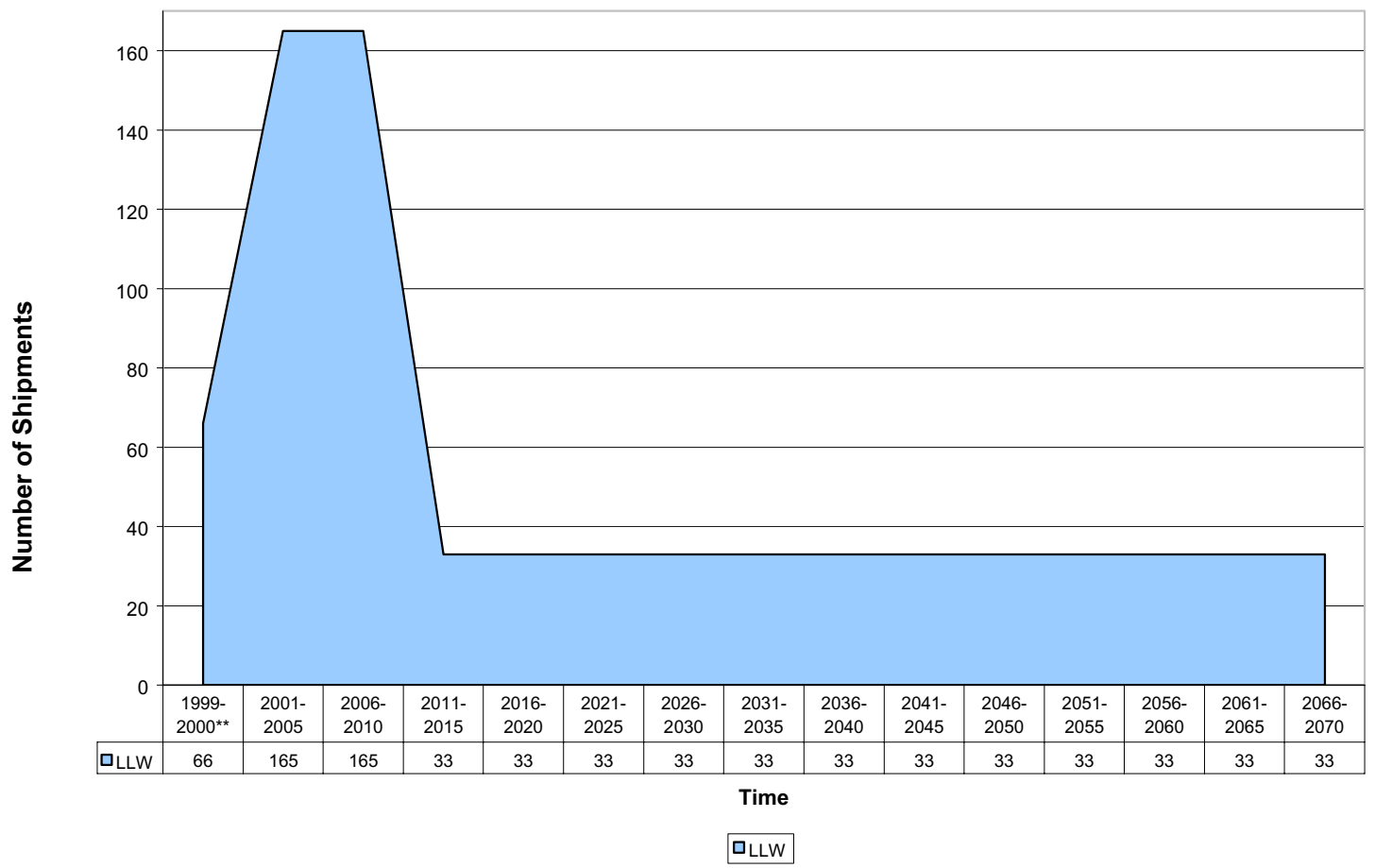

Total life-cycle values per 7/30/99 Stream Disposition Data (SDD) (http://id.inel.gov/avs/).

** Represents data for a two year period. 
Hanford

Incoming LLW Shipments from

Paducah Gaseous Diffusion Plant

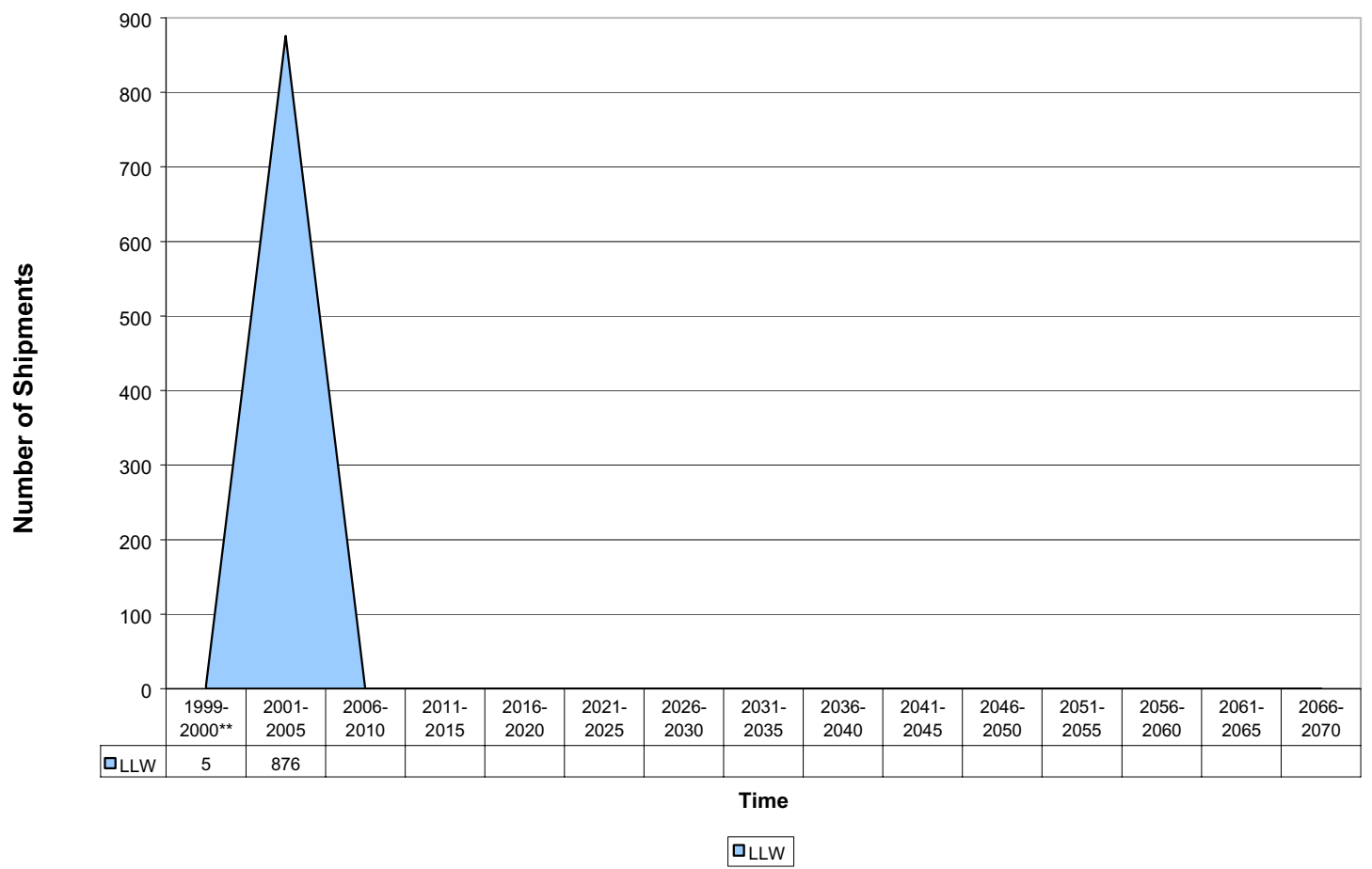

Hanford

Incoming LLW Shipments from AMES

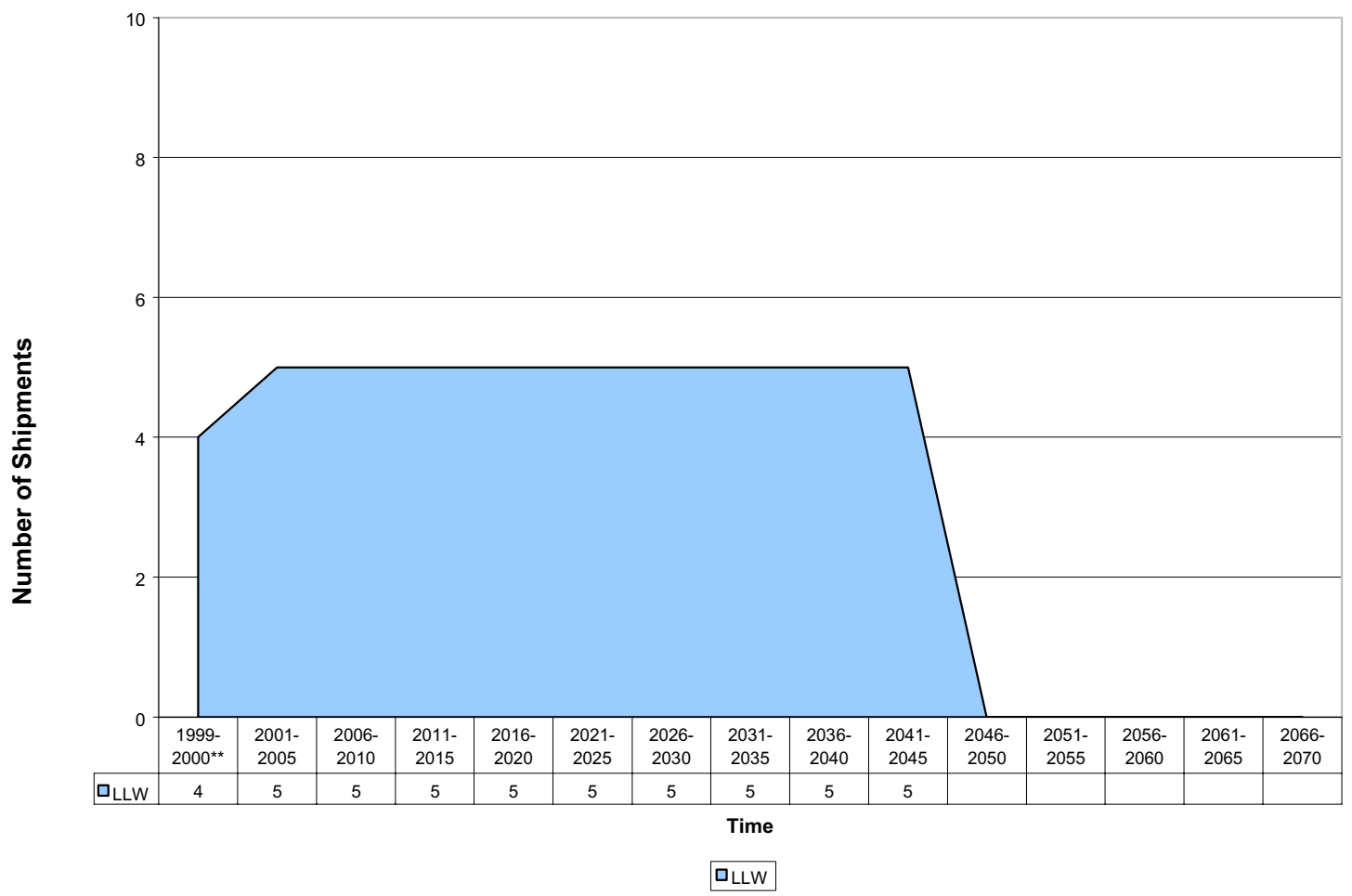

Total life-cycle values per 7/30/99 Stream Disposition Data (SDD) (http://id.inel.gov/avs/).

** Represents data for a two year period. 


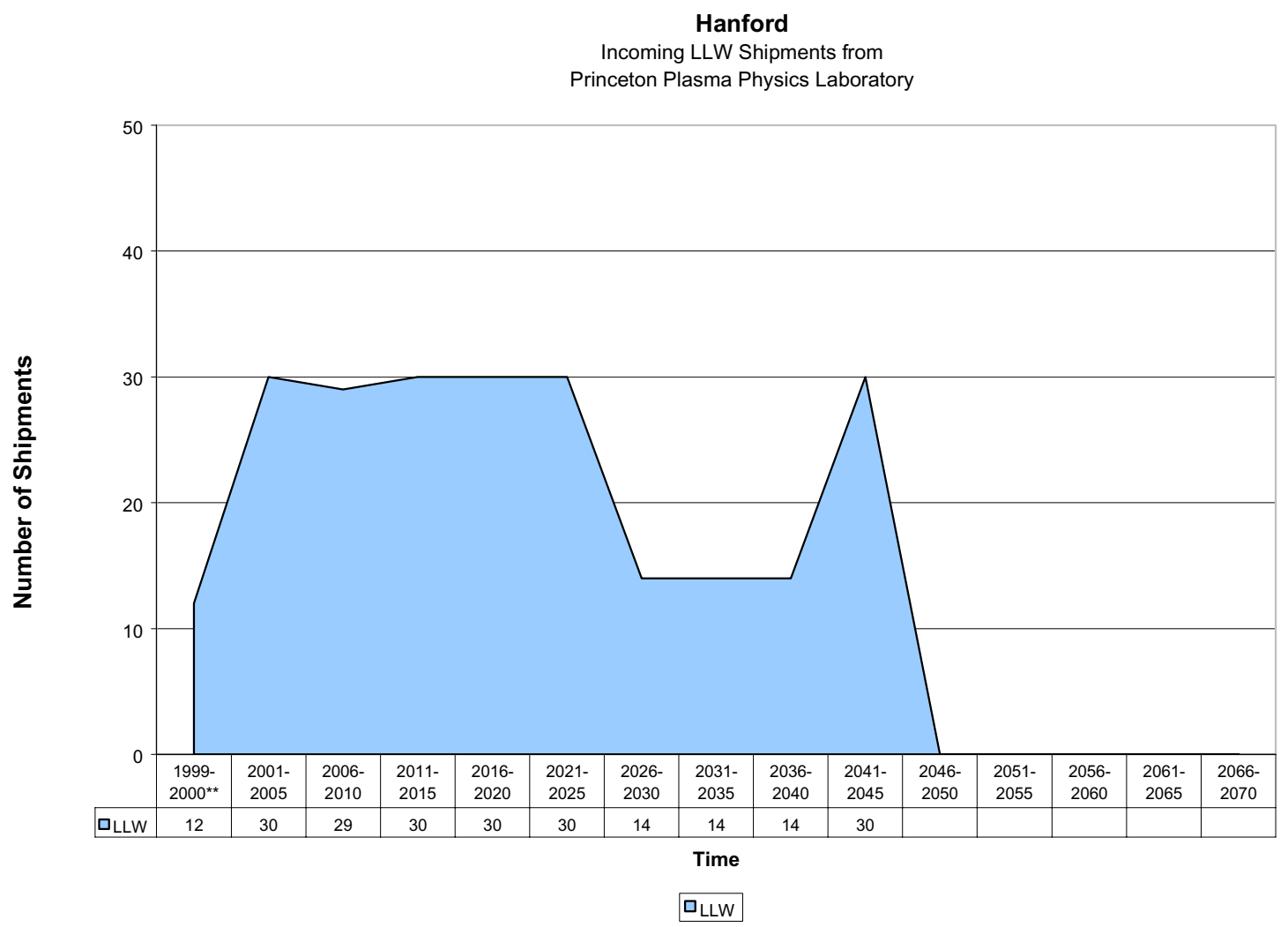

Hanford

Incoming LLW Shipments from

Brookhaven National Laboratory

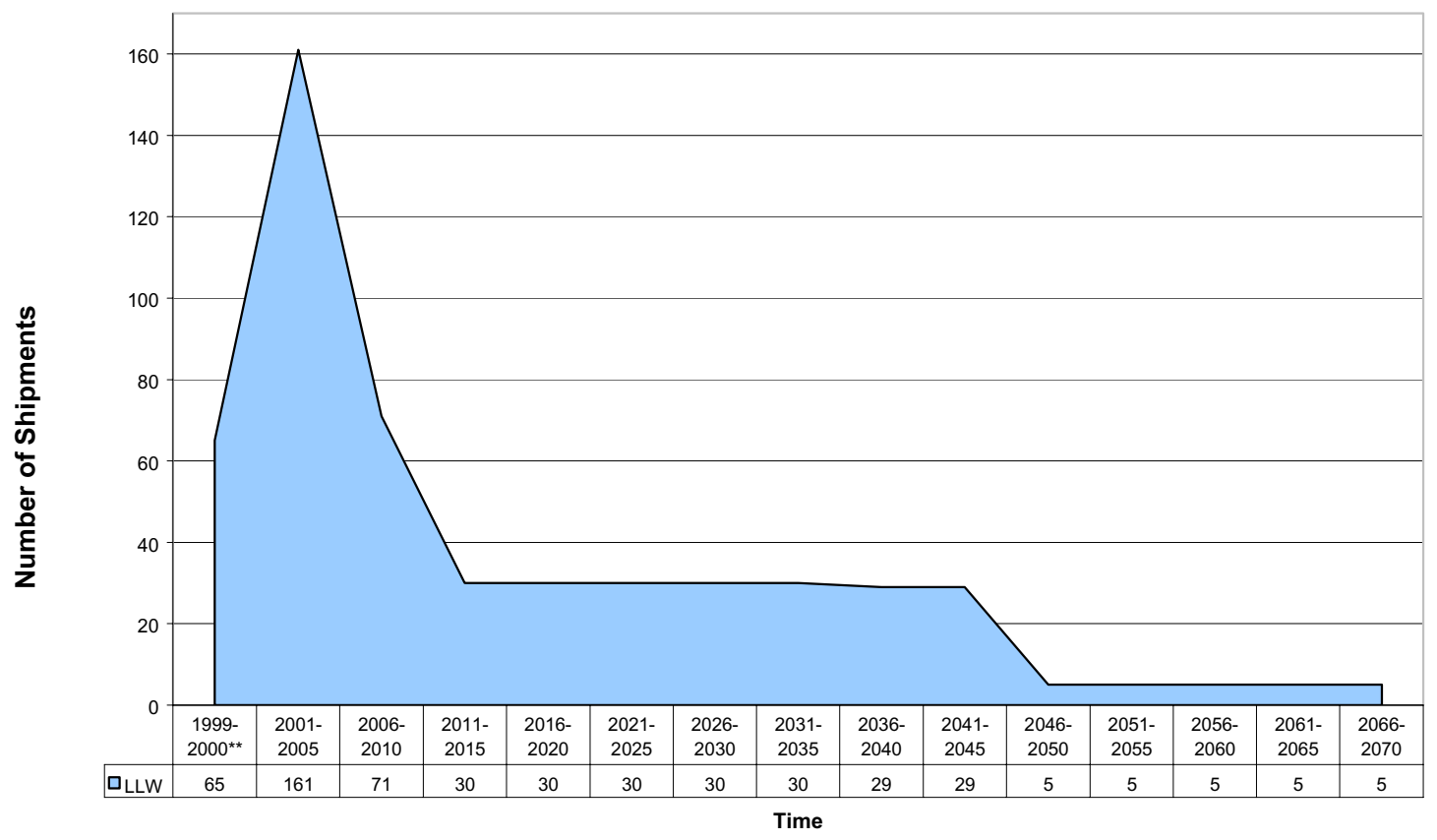

QLLW

Total life-cycle values per 7/30/99 Stream Disposition Data (SDD) (http://id.inel.gov/avs/).

** Represents data for a two year period. 
Hanford

Waste Stream Disposition

Life-cycle Incoming Volume

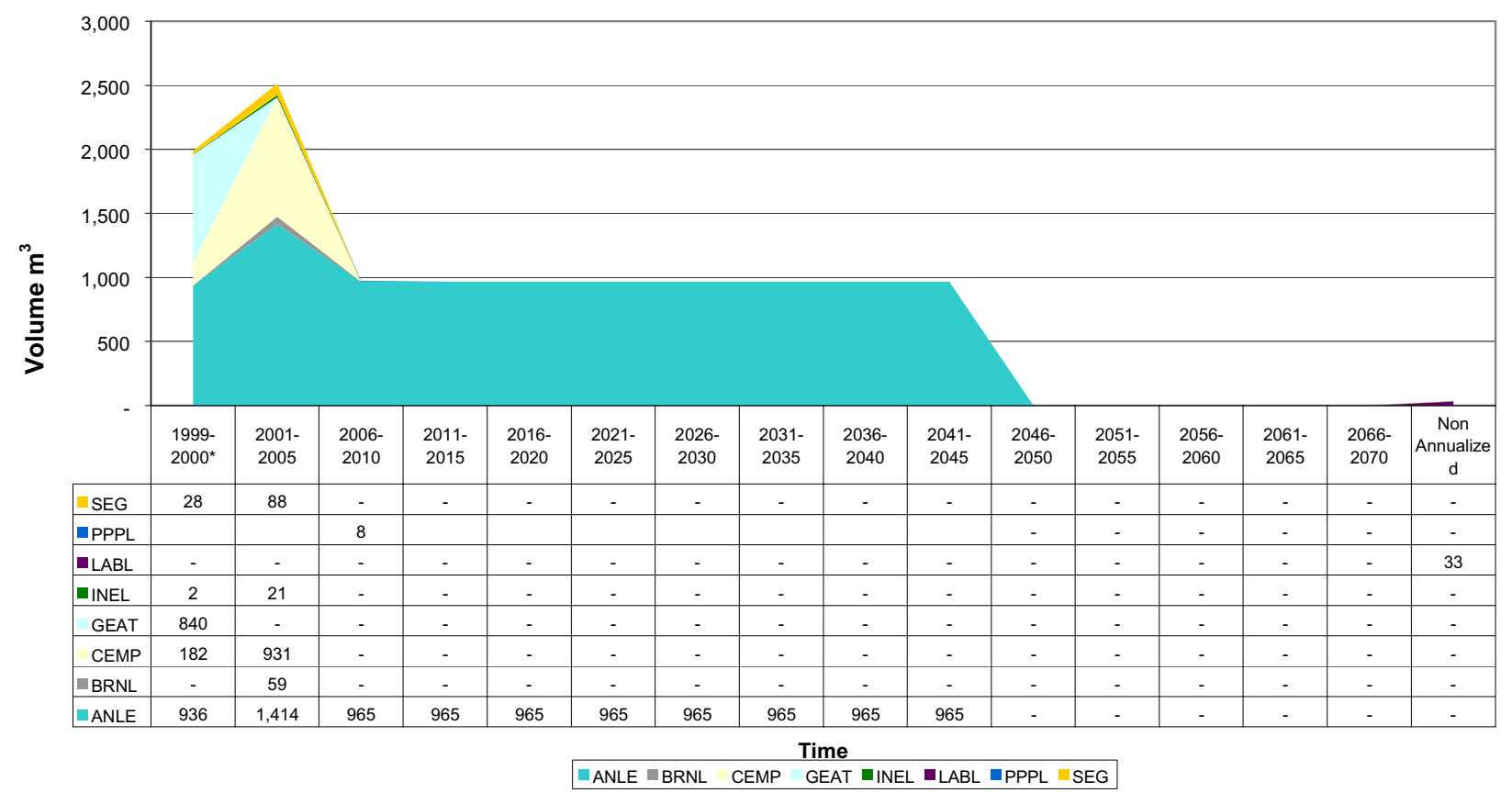

Hanford

Incoming LLW Volume from Argonne National Laboratory East

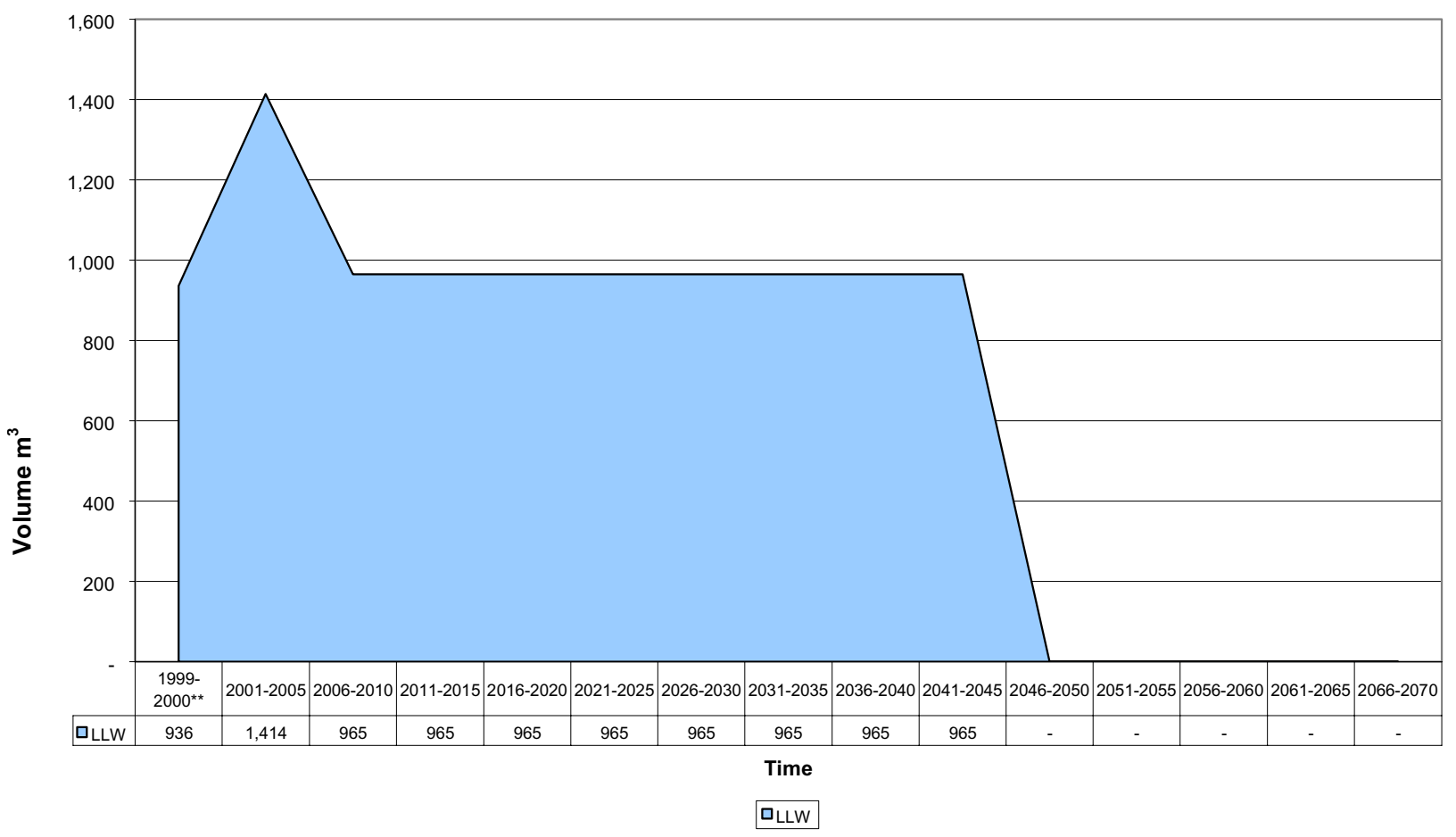

Total life-cycle values per 7/30/99 Stream Disposition Data (SDD) (http://id.inel.gov/avs/).

A-16

Printed: March 30, 2000

** Represents data for a two year period. 
Hanford

Incoming LLW Volume from Brookhaven National Laboratory

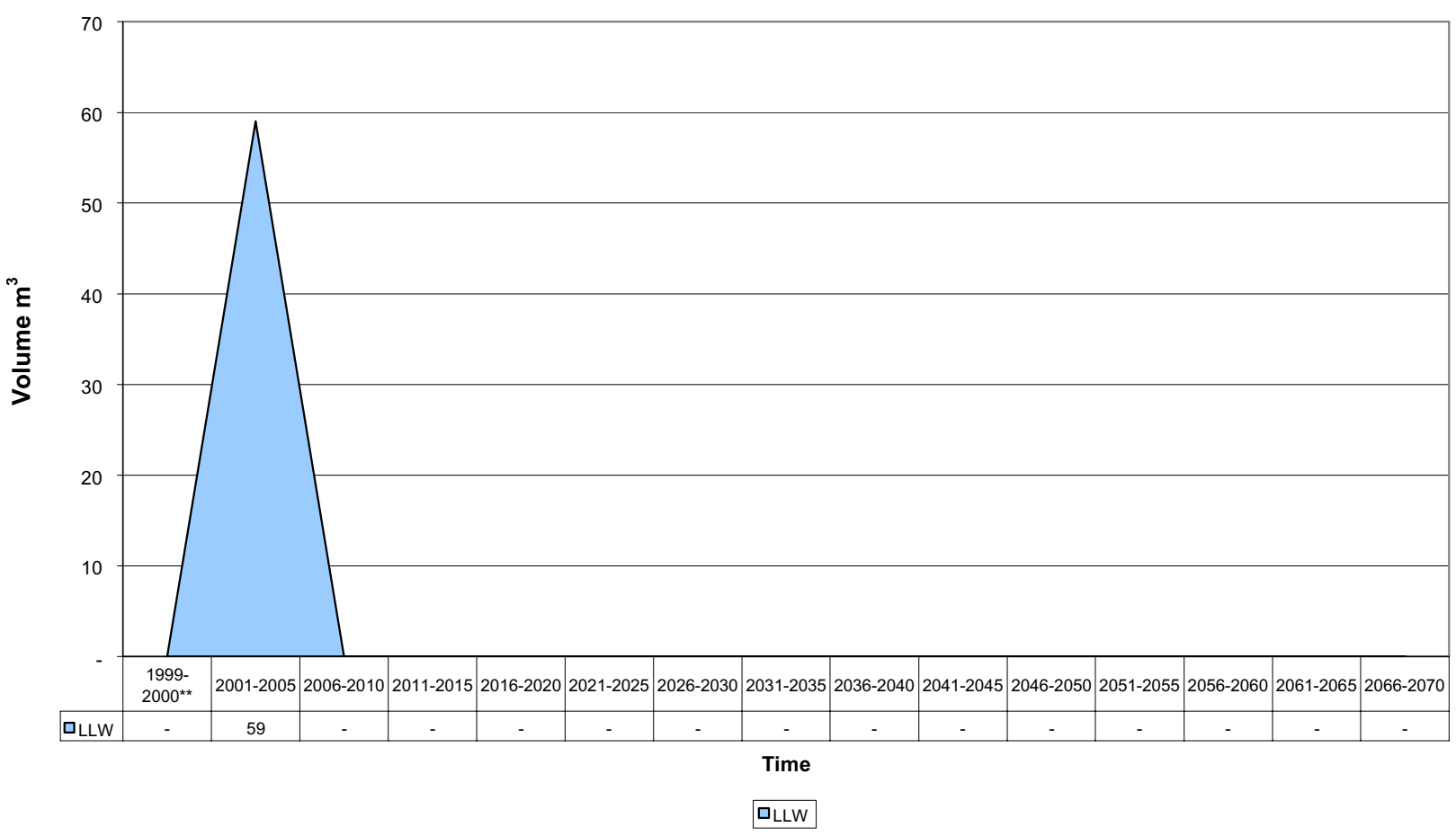

Hanford

Incoming M/LLW Volume from Columbus Environmental Management Project (WJ)

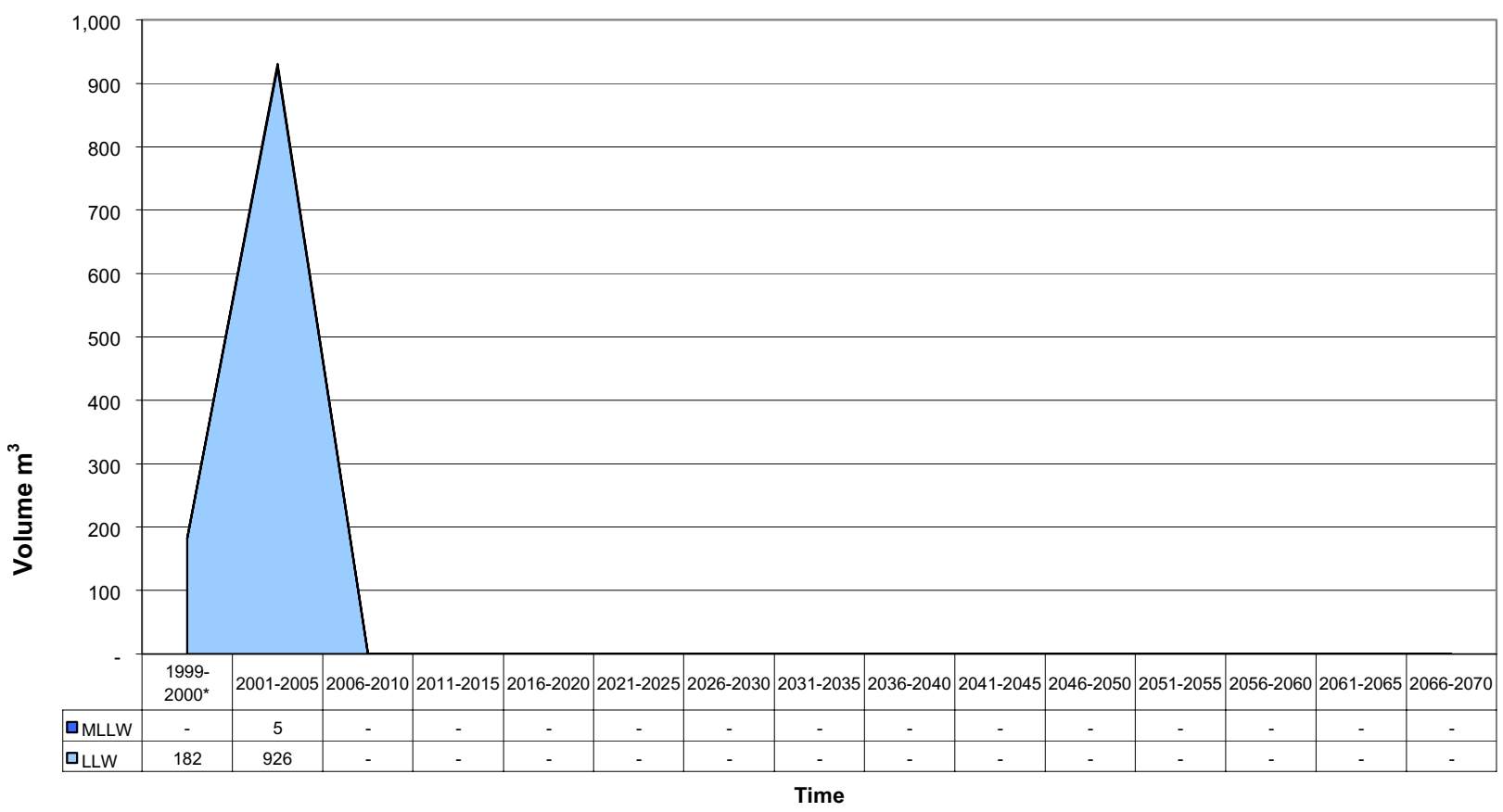

口LLW $\square$ MLLW

Total life-cycle values per 7/30/99 Stream Disposition Data (SDD) (http://id.inel.gov/avs/).

** Represents data for a two year period. 
Hanford

Incoming LLW Volume from General Atomics

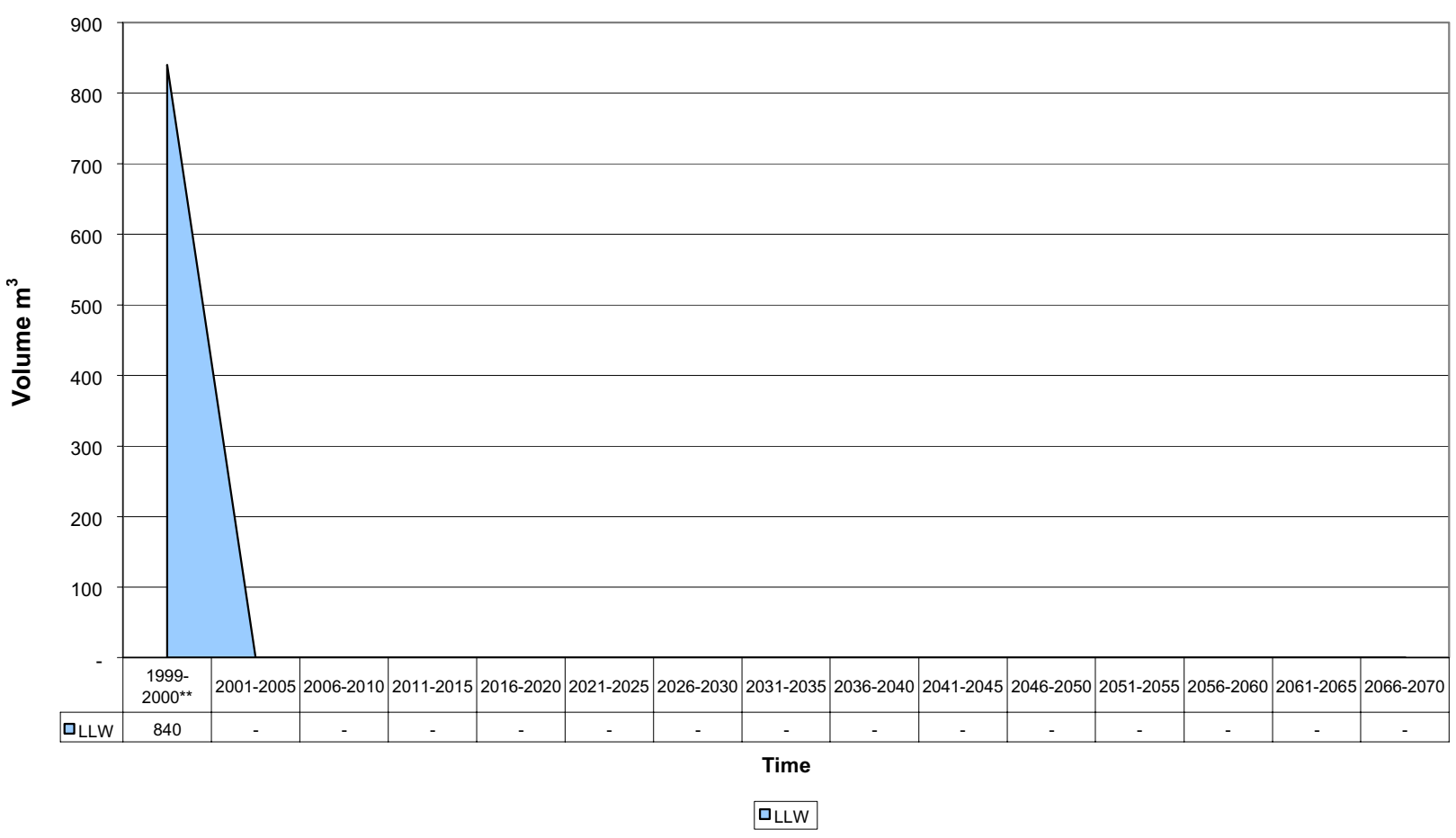

Hanford

Incoming MLLW Volume from INEEL

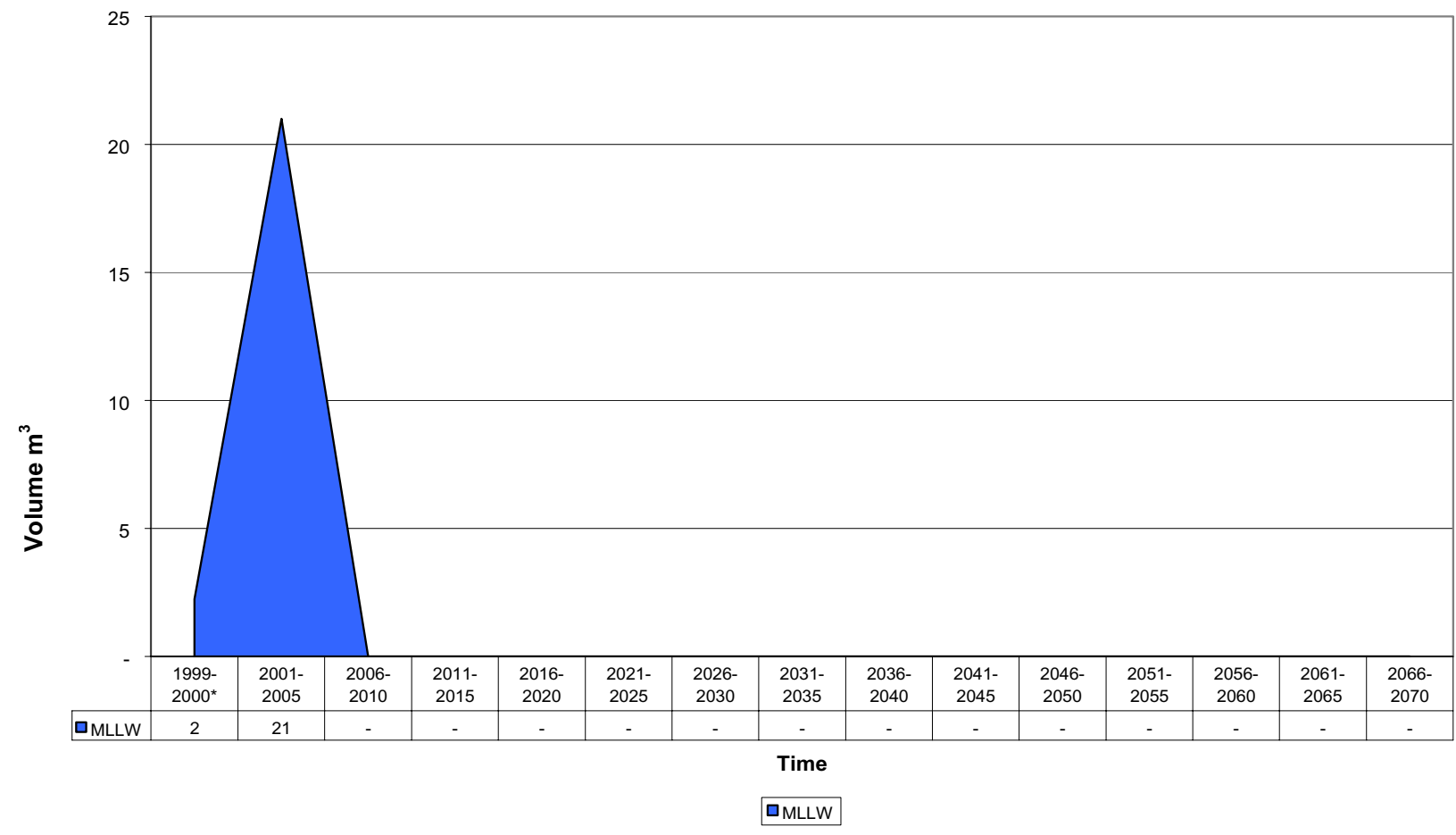

Total life-cycle values per 7/30/99 Stream Disposition Data (SDD) (http://id.inel.gov/avs/).

** Represents data for a two year period. 


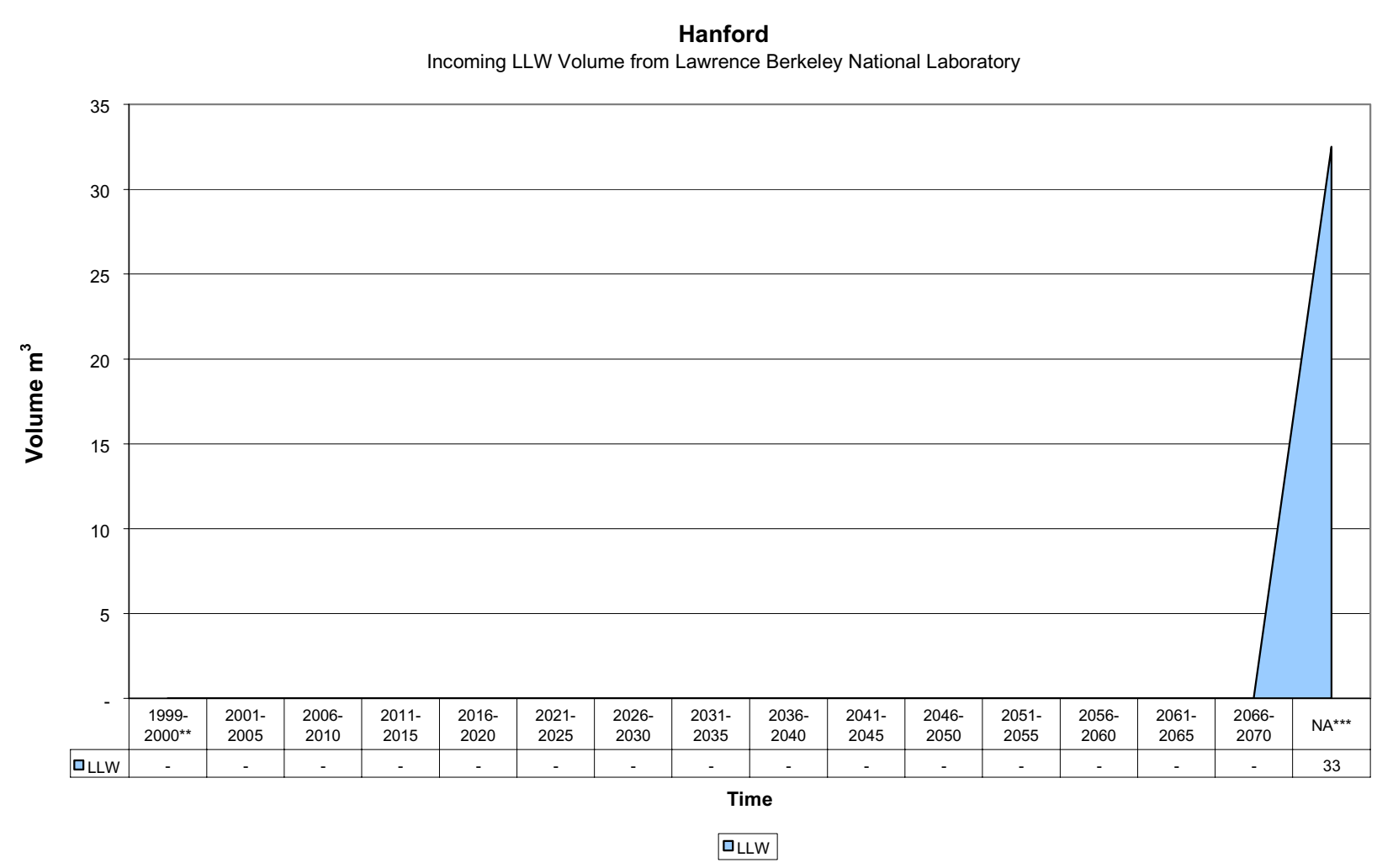

Hanford

Incoming LLW Volume from Princeton Plasma Physics Laboratory

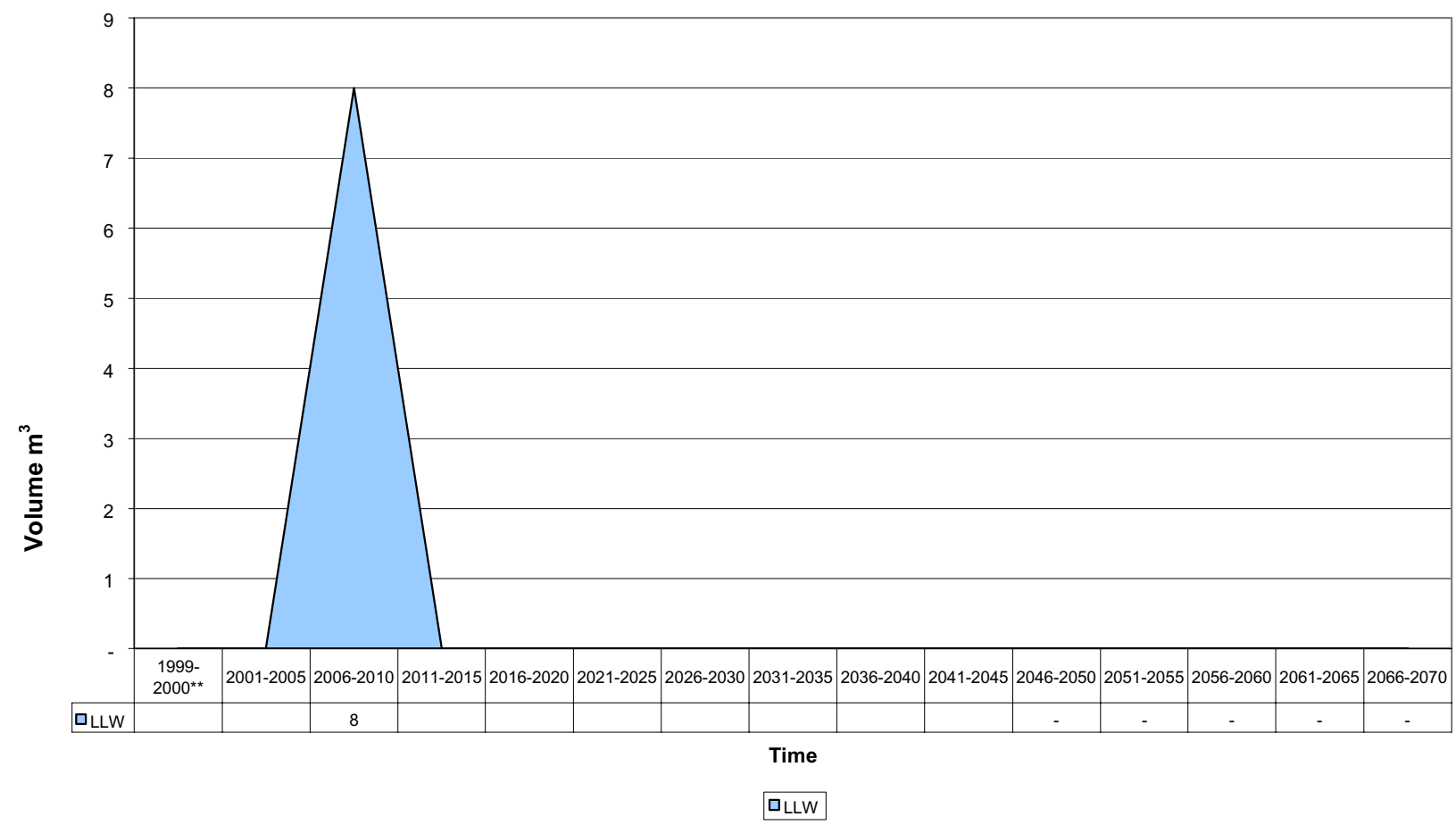

Total life-cycle values per 7/30/99 Stream Disposition Data (SDD) (http://id.inel.gov/avs/).

** Represents data for a two year period. 
Hanford

Incoming LLW Volume from GTS Duratek (SEG)

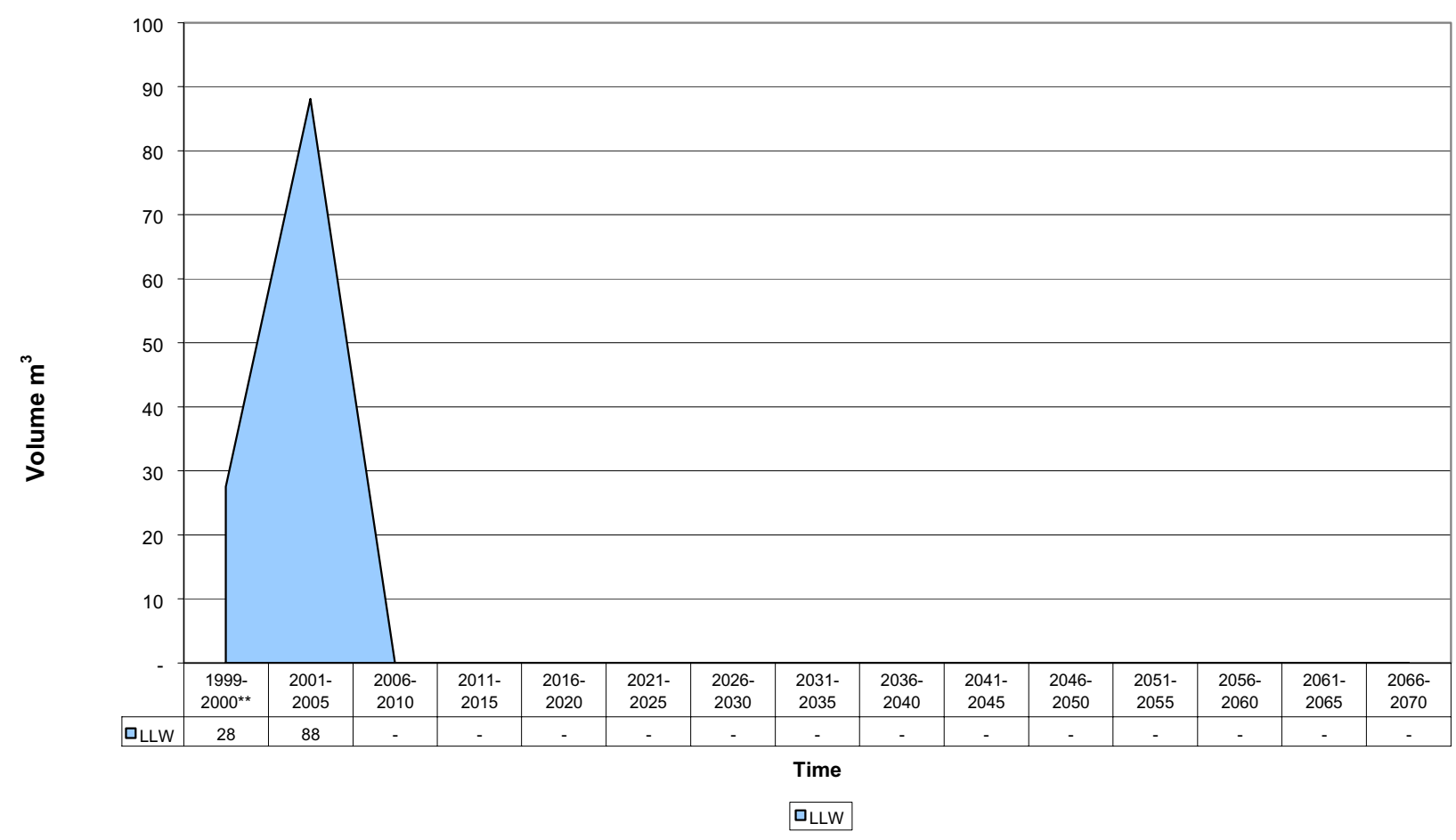

Total life-cycle values per 7/30/99 Stream Disposition Data (SDD) (http://id.inel.gov/avs/).

** Represents data for a two year period. 

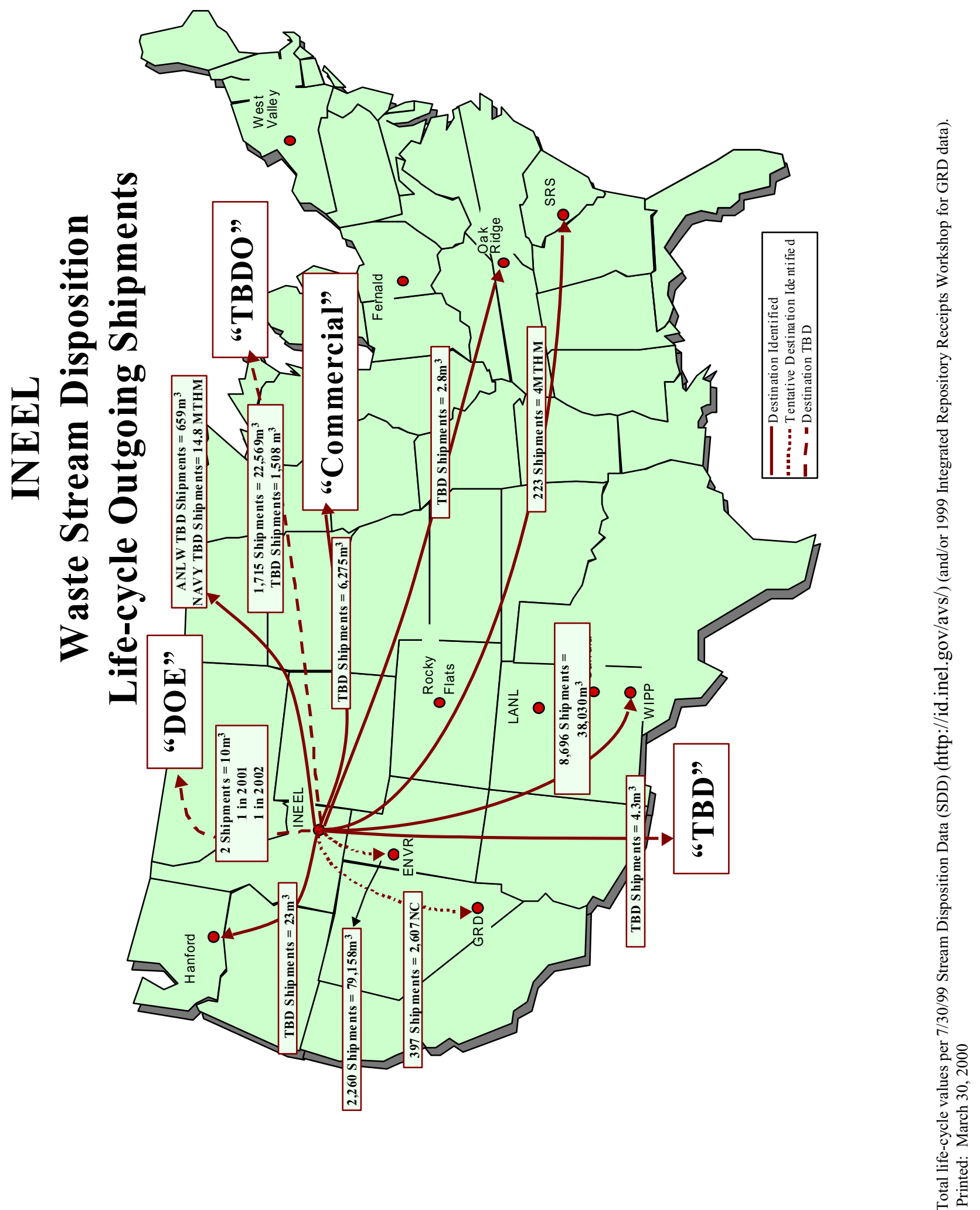
INEEL

Waste Stream Disposition Life-cycle Outgoing Shipments

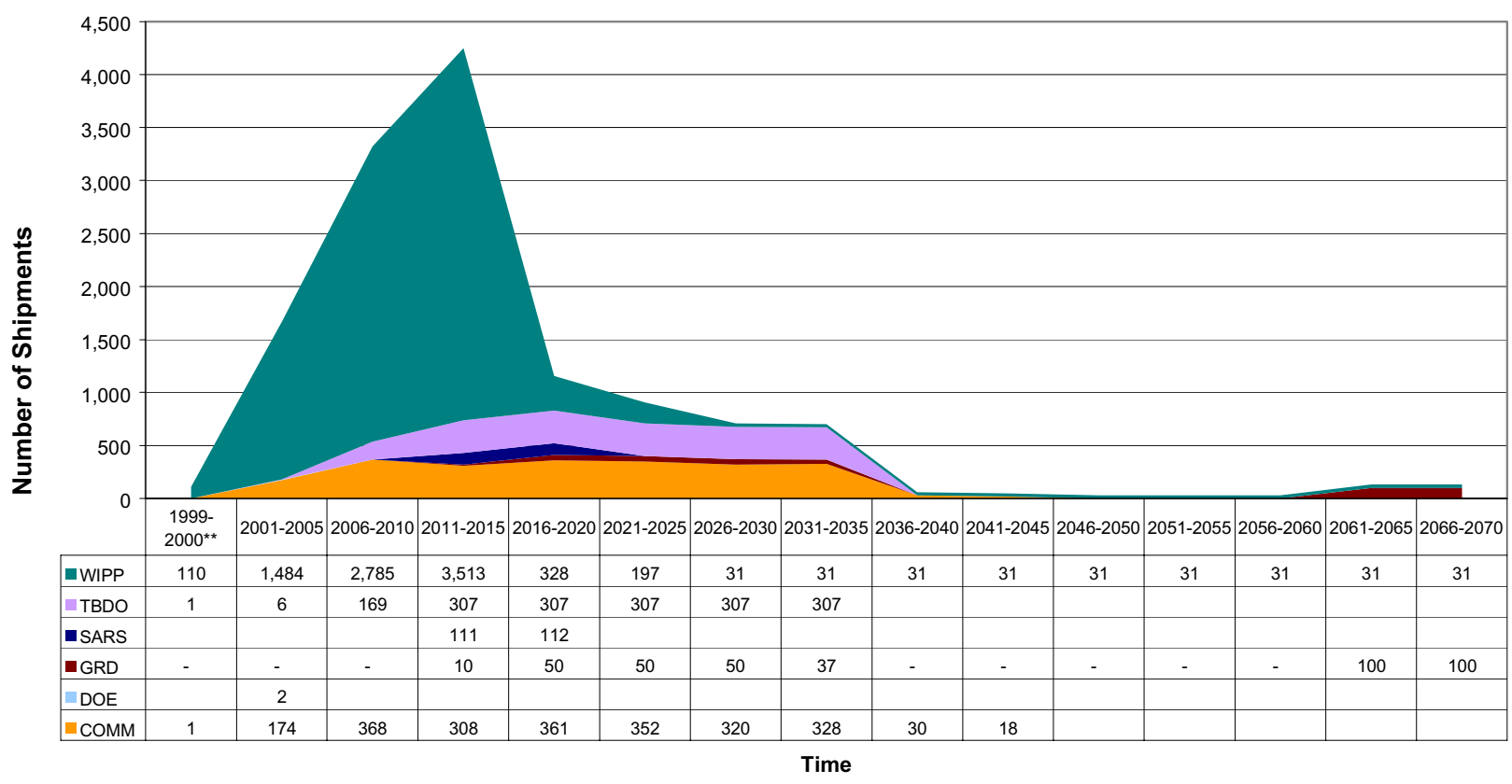

$\square \mathrm{COMM} \backsim \mathrm{DOE} \backsim \mathrm{GRD} \backsim \mathrm{SARS} \backsim \mathrm{TBDO} \backsim \mathrm{WIPP}$

INEEL

Outgoing HLW \& SNF Shipments to Geologic Repository Disposal (Tentatively Yucca Mtn.)

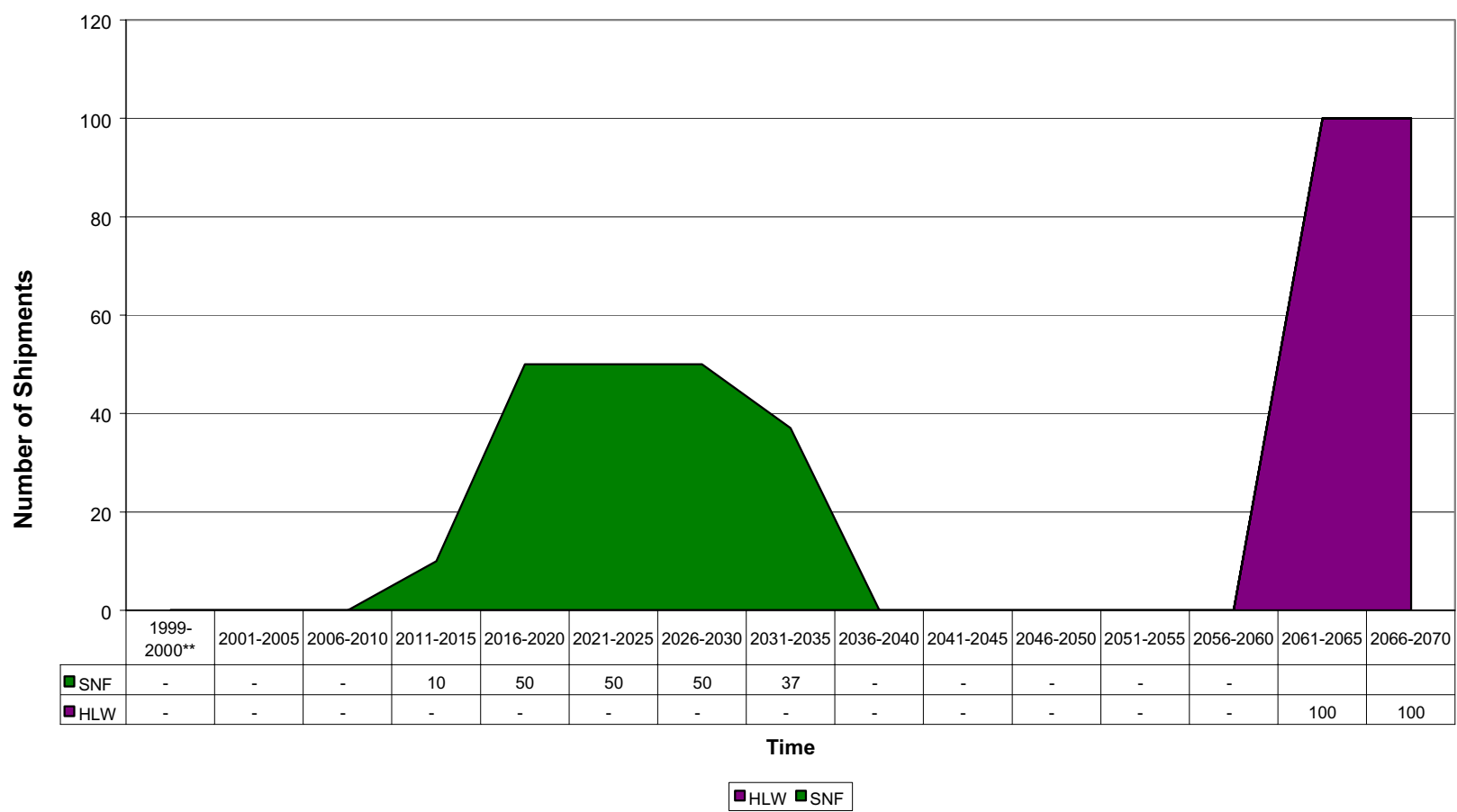

Total life-cycle values per 7/30/99 Stream Disposition Data (SDD) (http://id.inel.gov/avs/) (and/or 1999 Integrated Repository Receipts Workshop for GRD data).

Printed: March 30, 2000

** Represents data for a two year period. 
INEEL

Outgoing M/LLW Shipments to Commercial TSD

(Tentatively Envirocare)

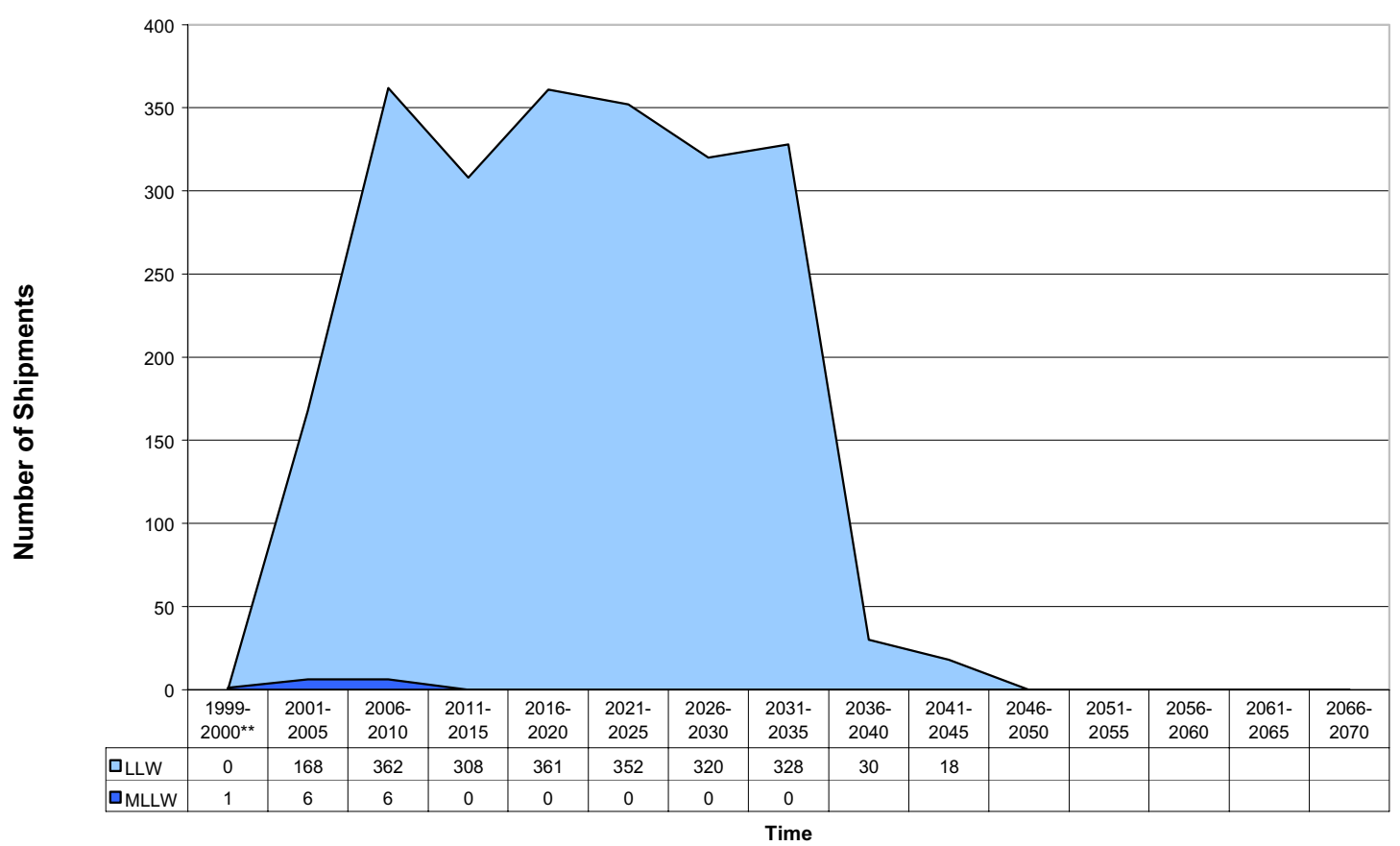

DLLW $\square_{M L L W}$

INEEL

Outgoing TRU Shipments to WIPP

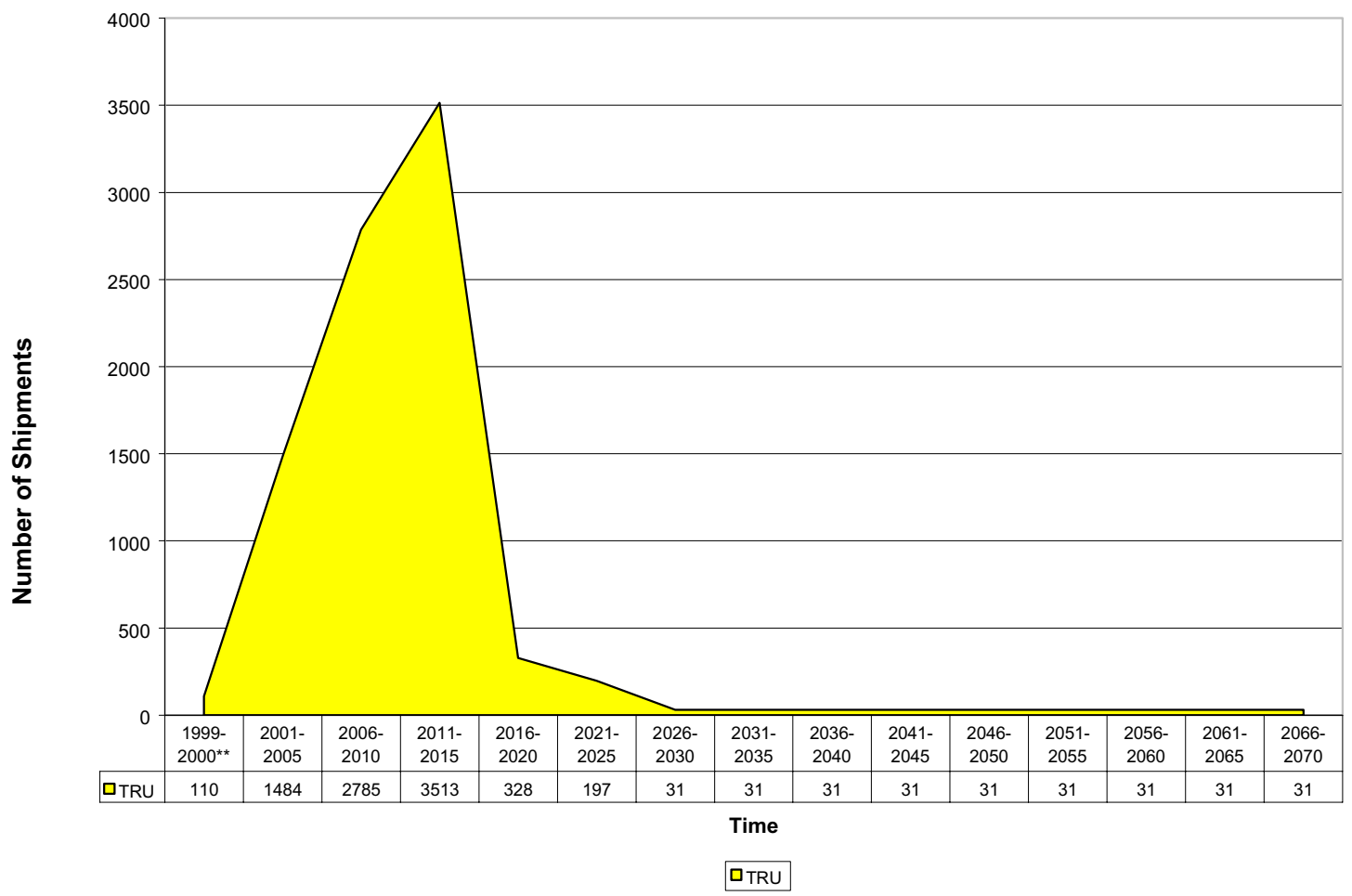

Total life-cycle values per 7/30/99 Stream Disposition Data (SDD) (http://id.inel.gov/avs/) (and/or 1999 Integrated Repository Receipts Workshop for GRD data).

Printed: March 30, 2000

** Represents data for a two year period. 
INEEL

Outgoing SNF Shipments to

Savannah River Site

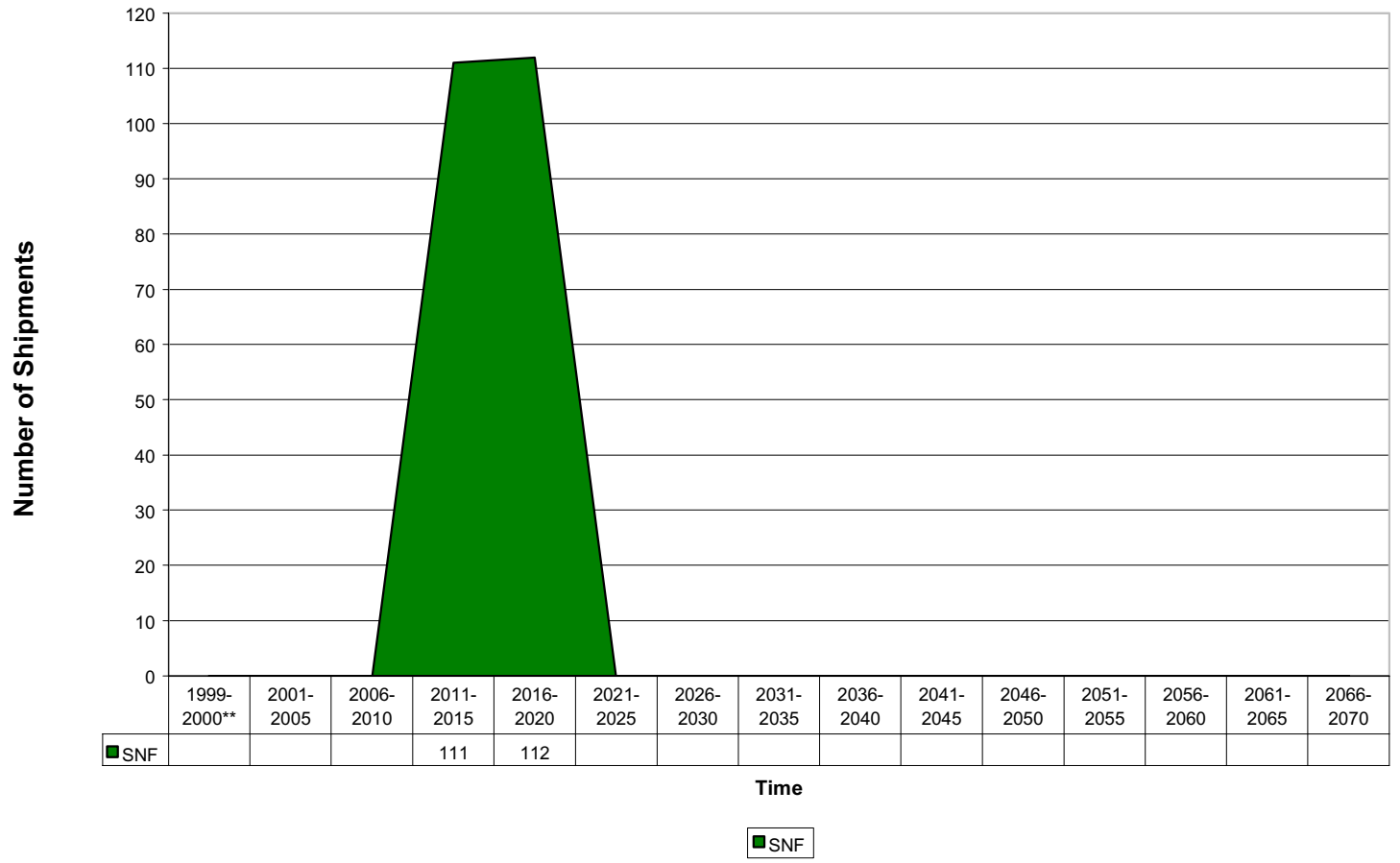

INEEL

Outgoing M/LLW Shipments to "TBDO"

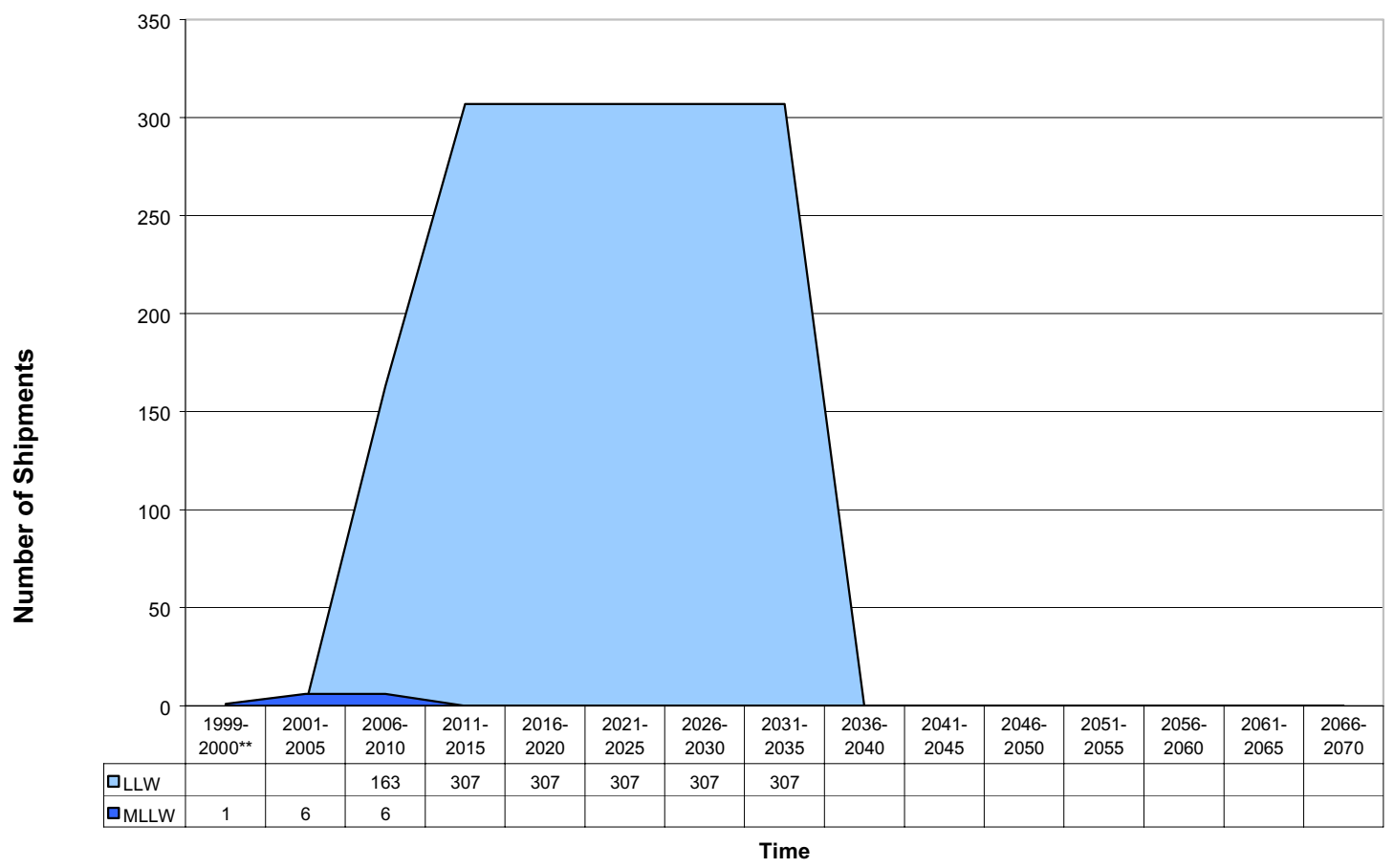

QLLW QMLLW

Total life-cycle values per 7/30/99 Stream Disposition Data (SDD) (http://id.inel.gov/avs/) (and/or 1999 Integrated Repository Receipts Workshop for GRD data).

Printed: March 30, 2000

** Represents data for a two year period. 
INEEL

Waste Stream Disposition Life-cycle Outgoing Volume

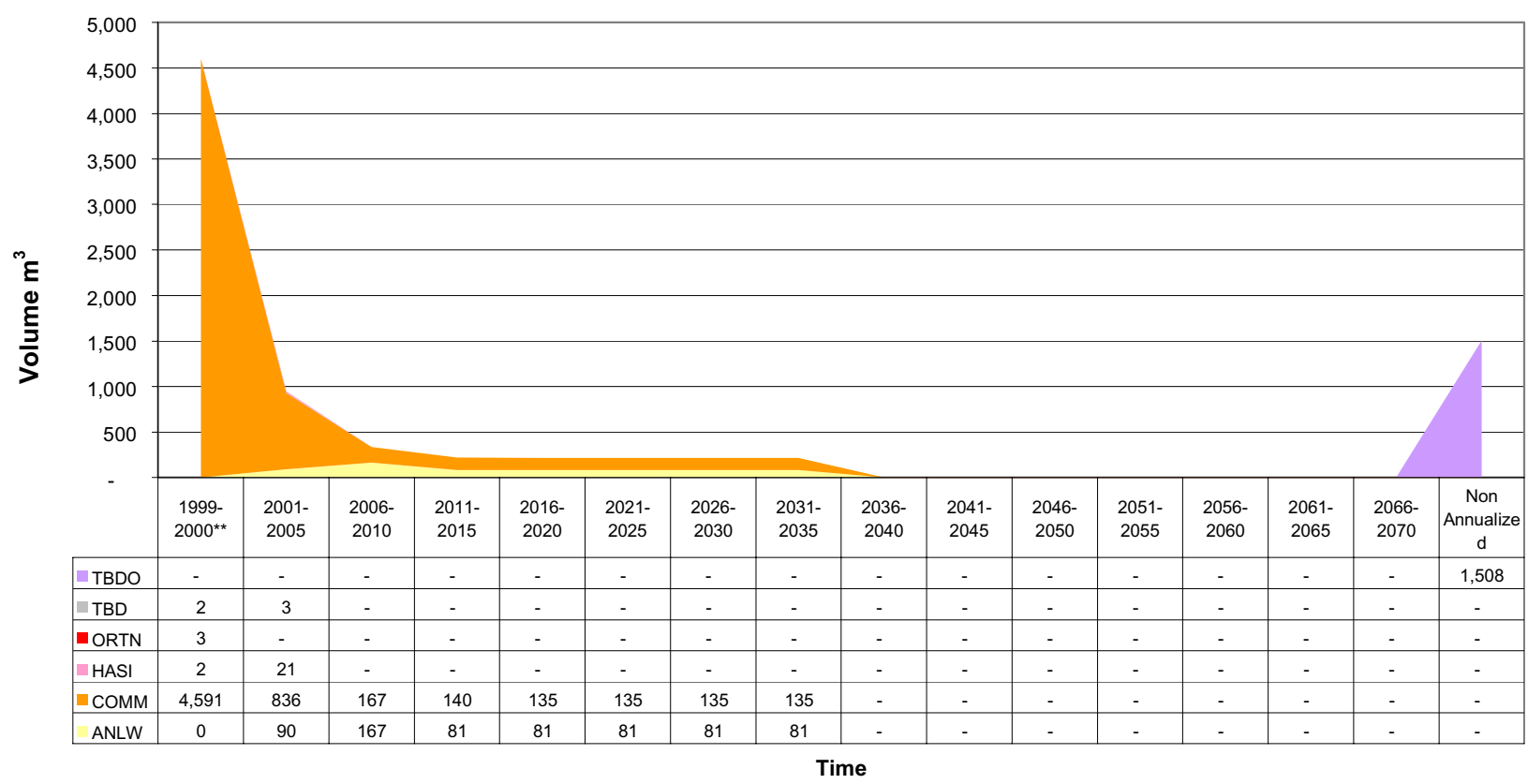

ANLW $₫$ COMM $₫$ HASI $₫$ ORTN $\backsim$ TBD $₫$ TBDO

INEEL

Outgoing MLLW Volume to Argonne National Laboratory West

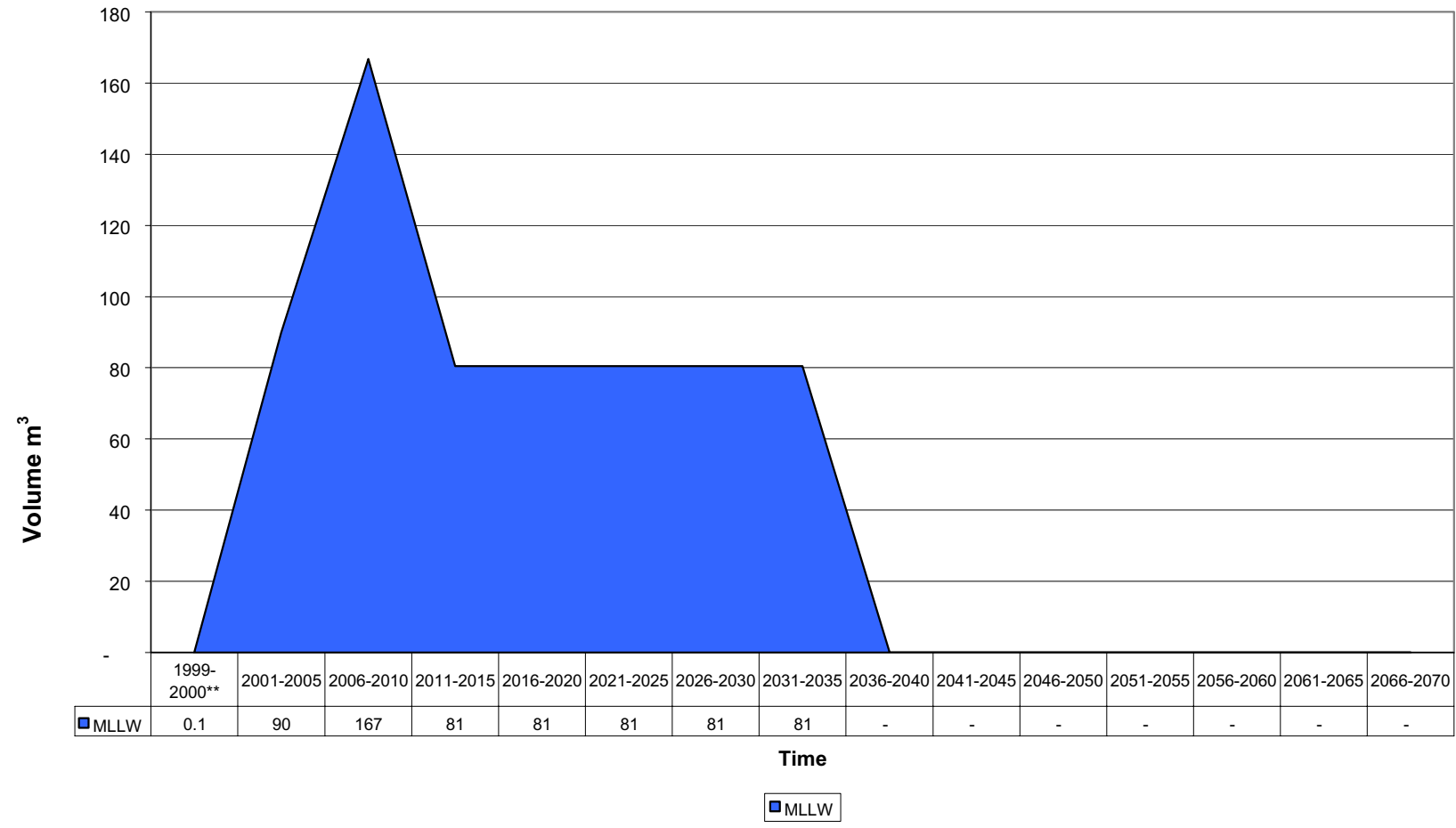

Total life-cycle values per 7/30/99 Stream Disposition Data (SDD) (http://id.inel.gov/avs/) (and/or 1999 Integrated Repository Receipts Workshop for GRD data).

Printed: March 30, 2000

** Represents data for a two year period. 
INEEL

Outgoing M/LLW Volume to "Commercial"

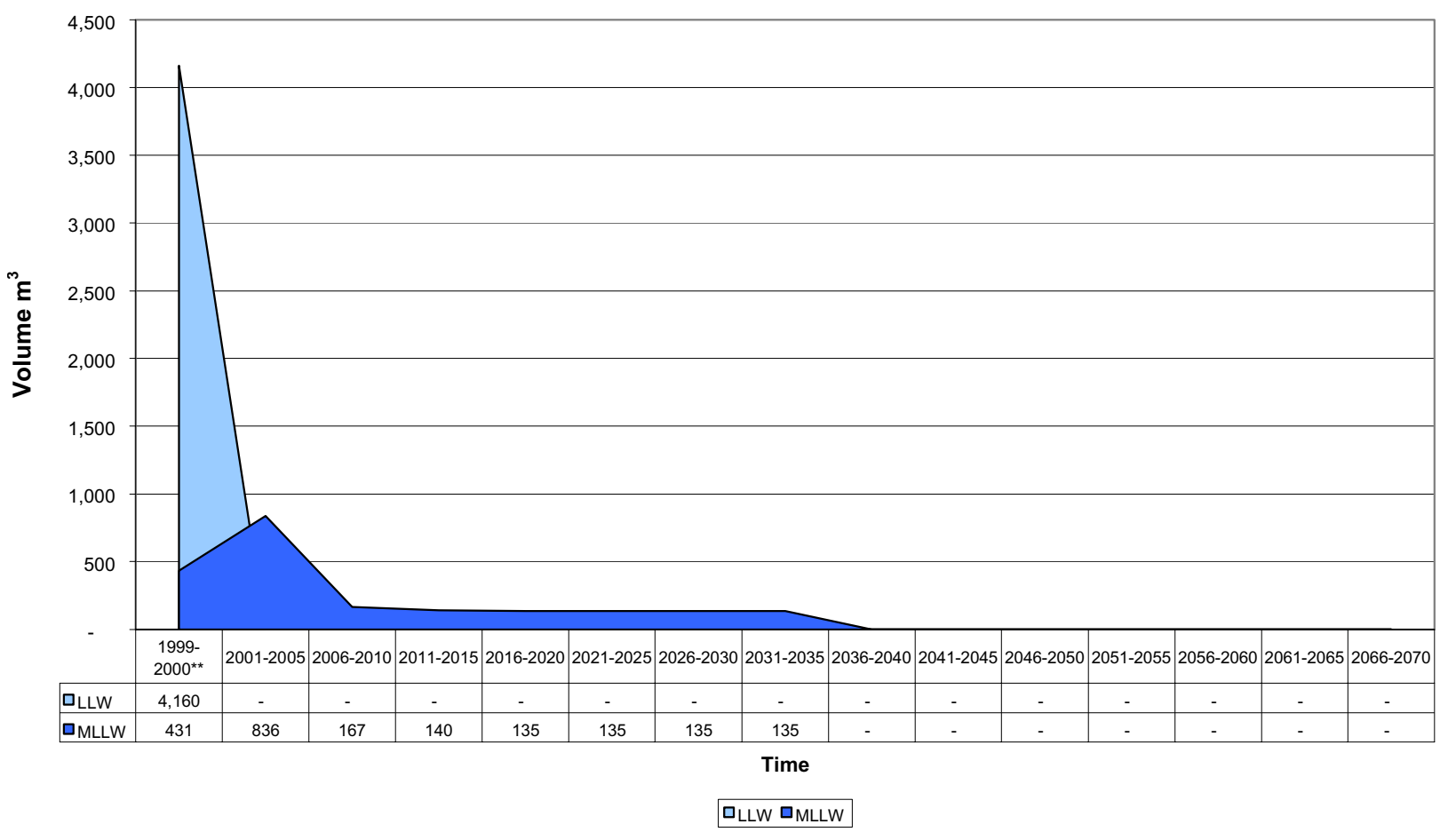

INEEL

Outgoing MLLW Volume to Hanford

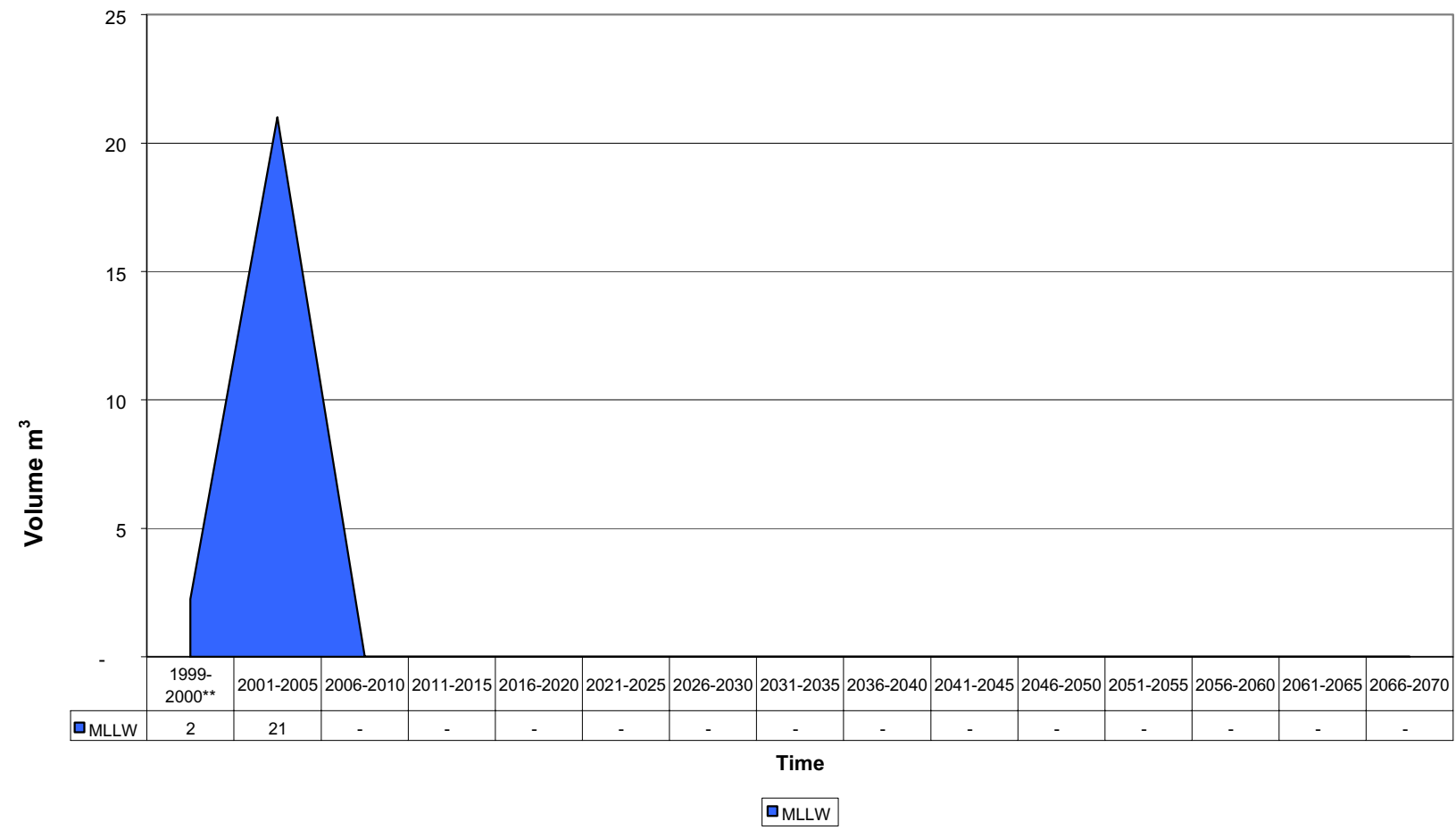

Total life-cycle values per 7/30/99 Stream Disposition Data (SDD) (http://id.inel.gov/avs/) (and/or 1999 Integrated Repository Receipts Workshop for GRD data).

Printed: March 30, 2000

** Represents data for a two year period. 
INEEL

Outgoing MLLW Volume to Oak Ridge

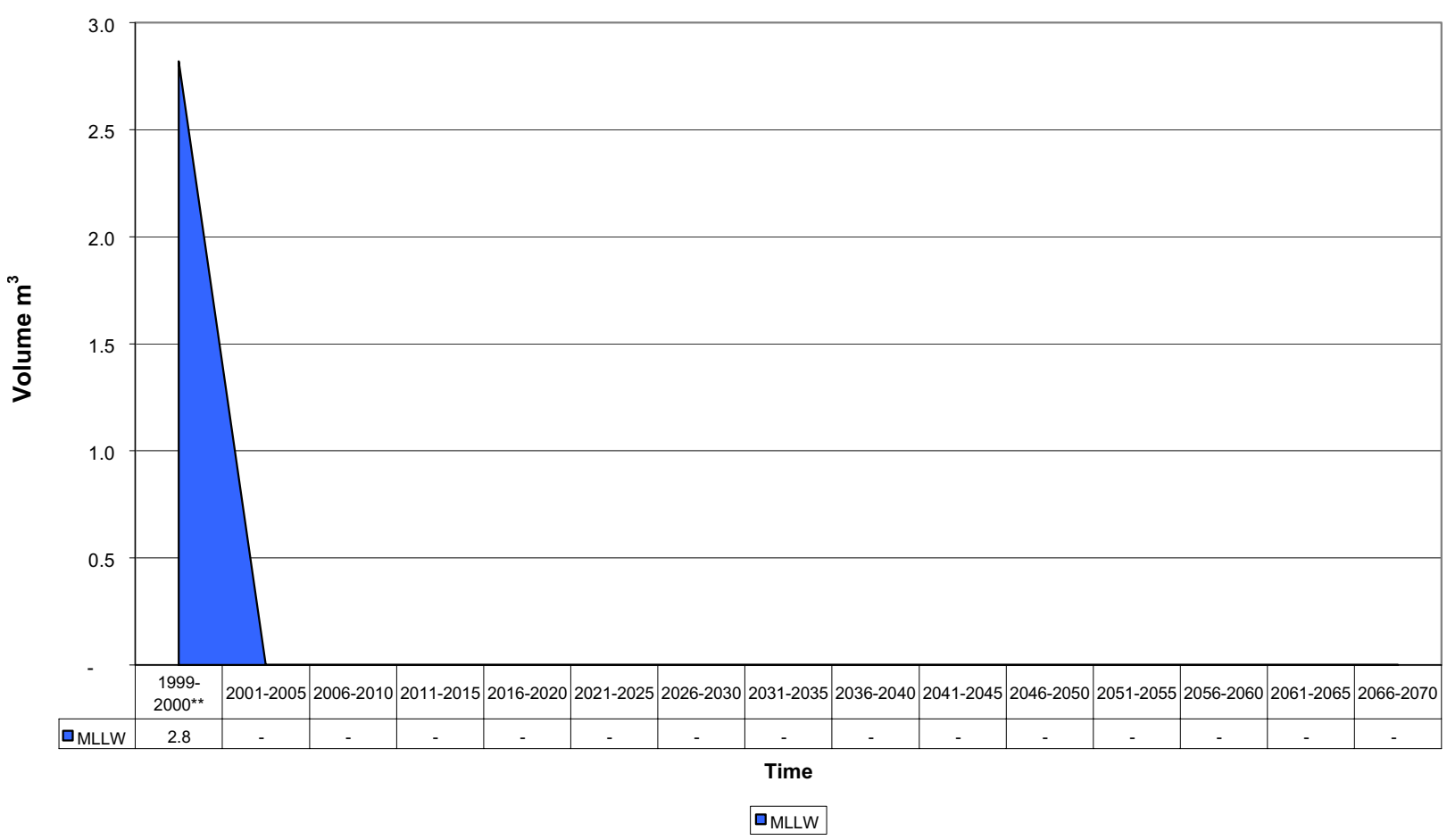

INEEL

Outgoing MLLW Volume to "TBD"

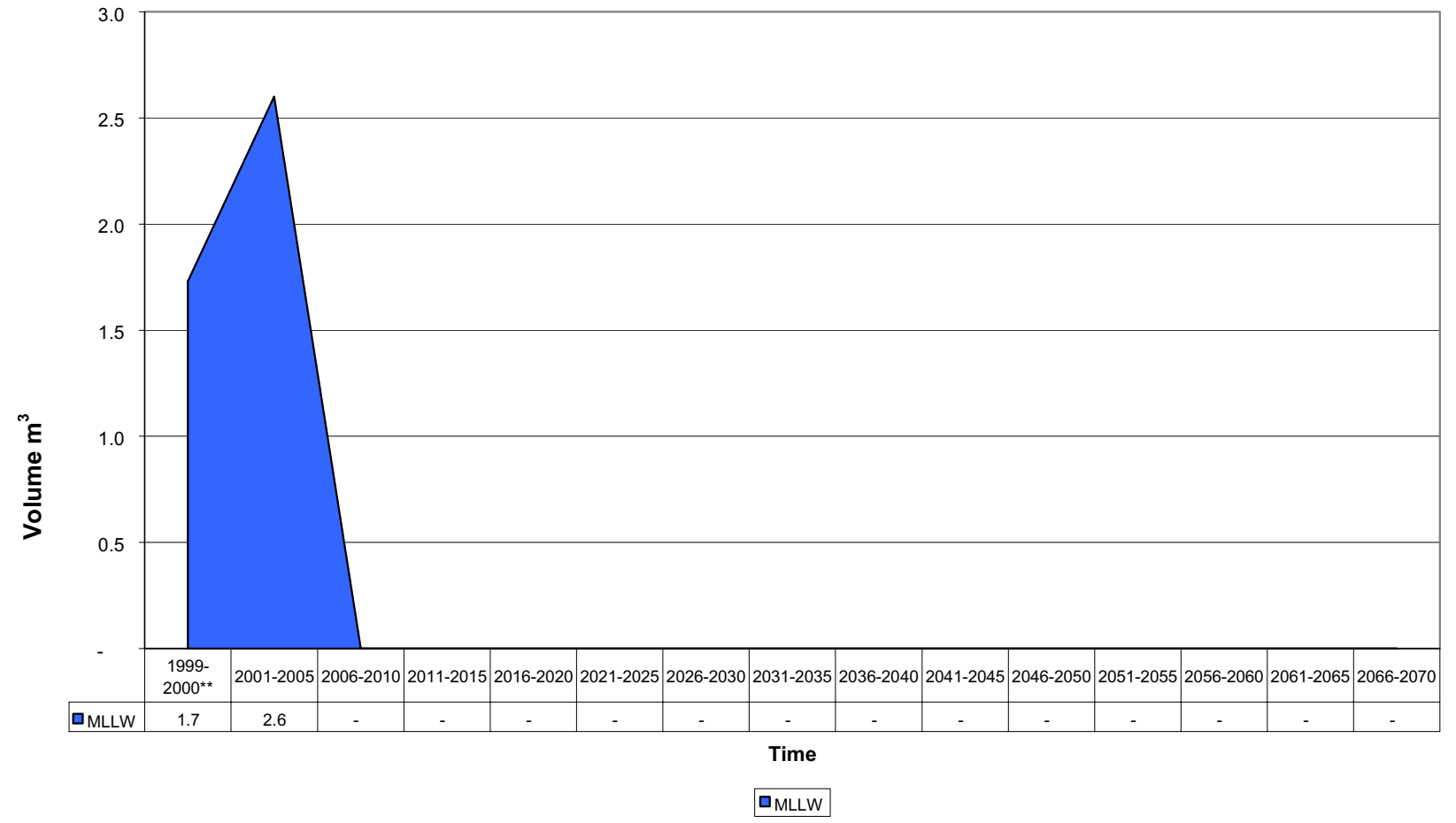

Total life-cycle values per 7/30/99 Stream Disposition Data (SDD) (http://id.inel.gov/avs/) (and/or 1999 Integrated Repository Receipts Workshop for GRD data).

Printed: March 30, 2000

** Represents data for a two year period. 
INEEL

Outgoing TRU Volume to "TBDO"

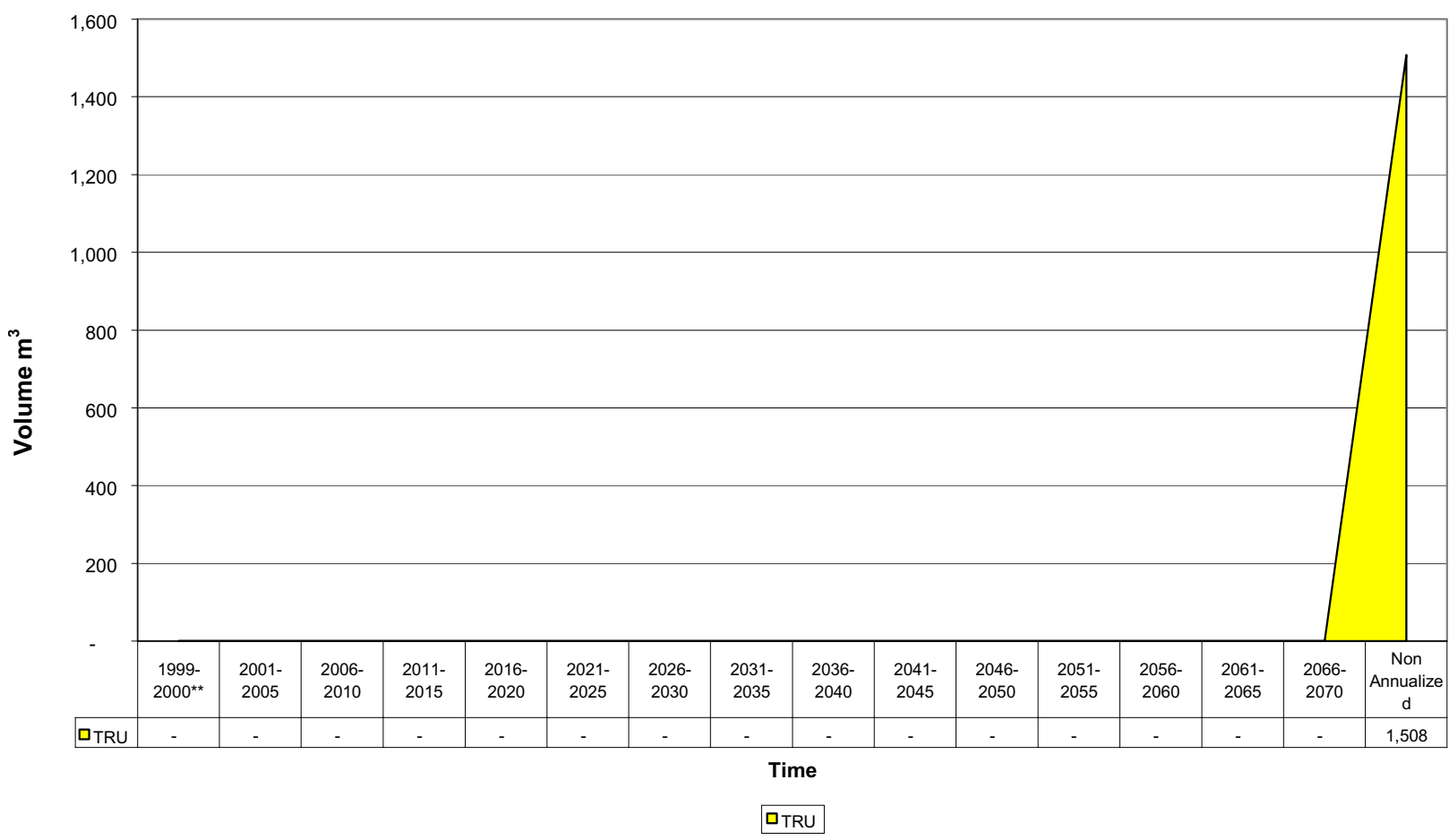

INEEL

Waste Stream Disposition Life-cycle Outgoing SNF

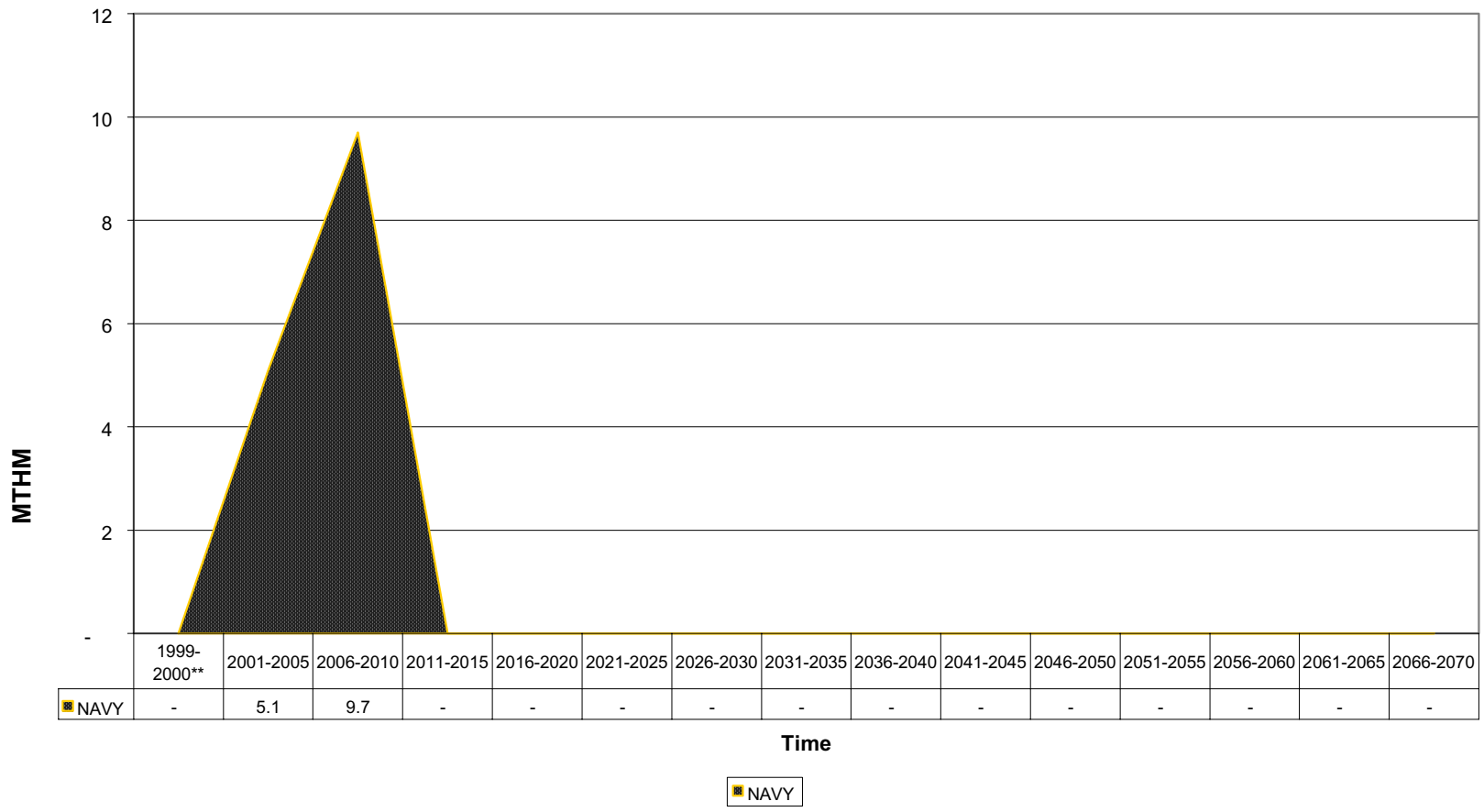

Total life-cycle values per 7/30/99 Stream Disposition Data (SDD) (http://id.inel.gov/avs/) (and/or 1999 Integrated Repository Receipts Workshop for GRD data).

Printed: March 30, 2000

** Represents data for a two year period. 
INEEL

Outgoing SNF to NAVY

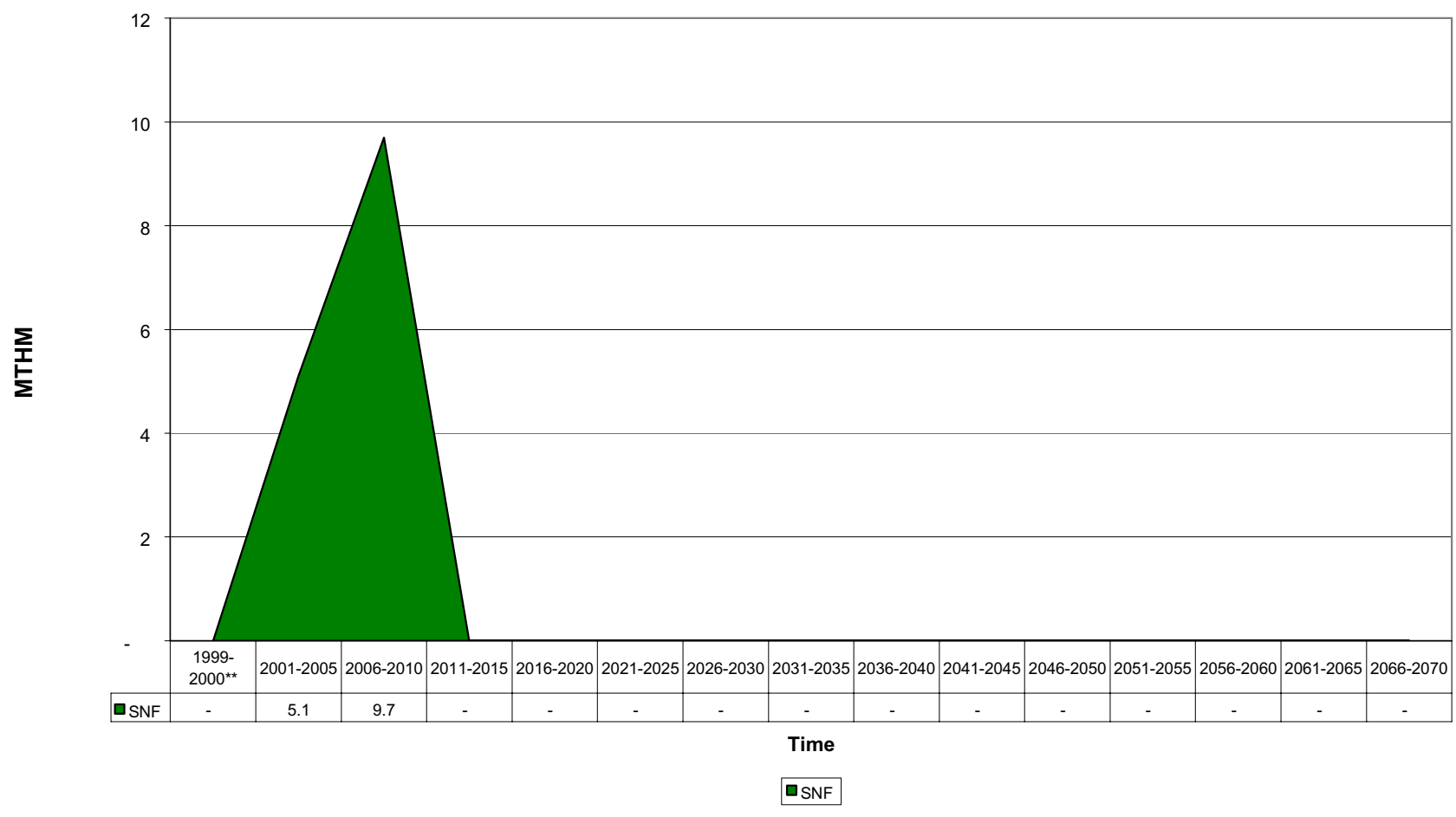

Total life-cycle values per 7/30/99 Stream Disposition Data (SDD) (http://id.inel.gov/avs/)

(and/or 1999 Integrated Repository Receipts Workshop for GRD data).

Printed: March 30, 2000

** Represents data for a two year period. 



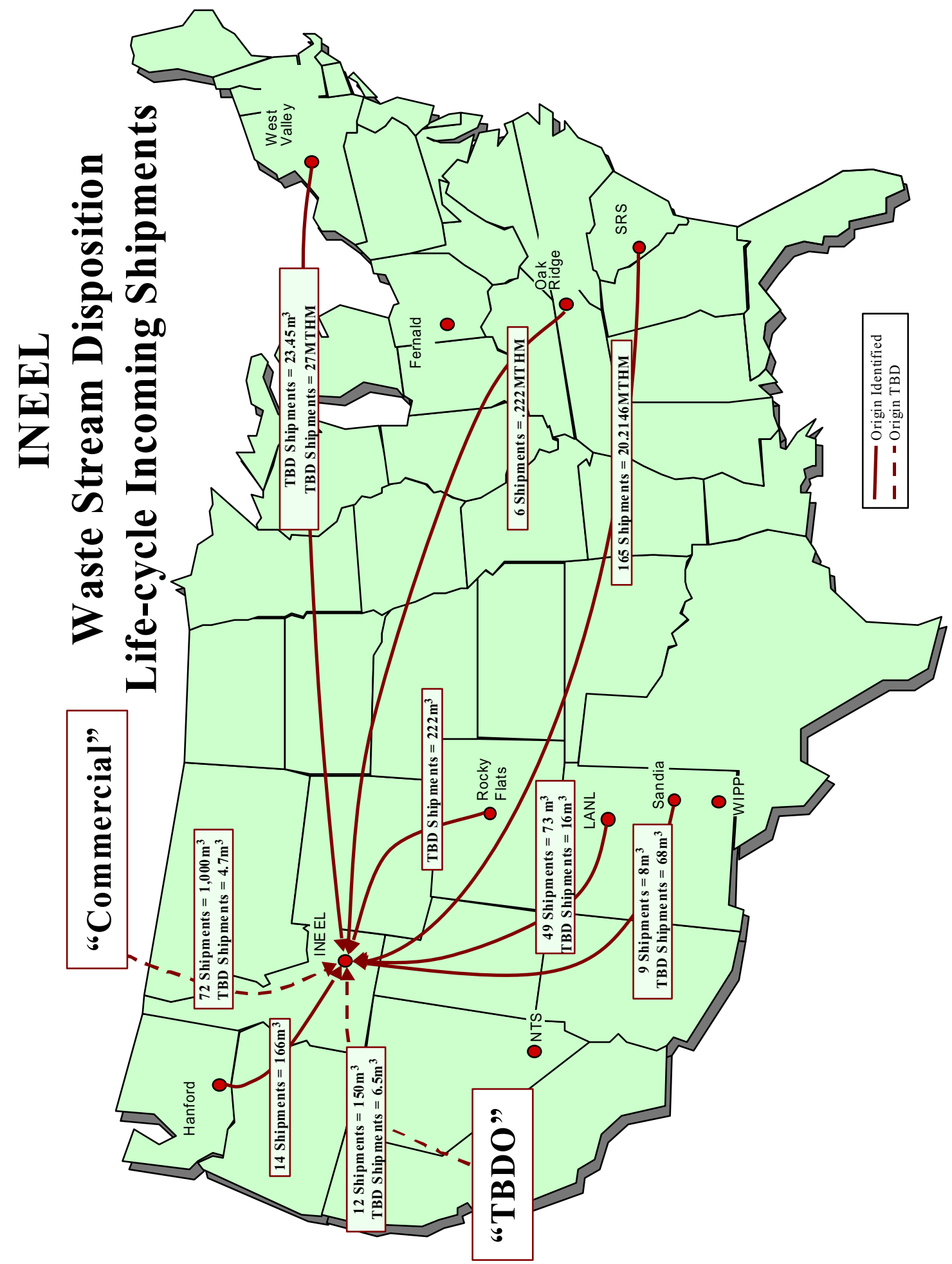


INEEL

Waste Stream Disposition Life-cycle Incoming Shipments

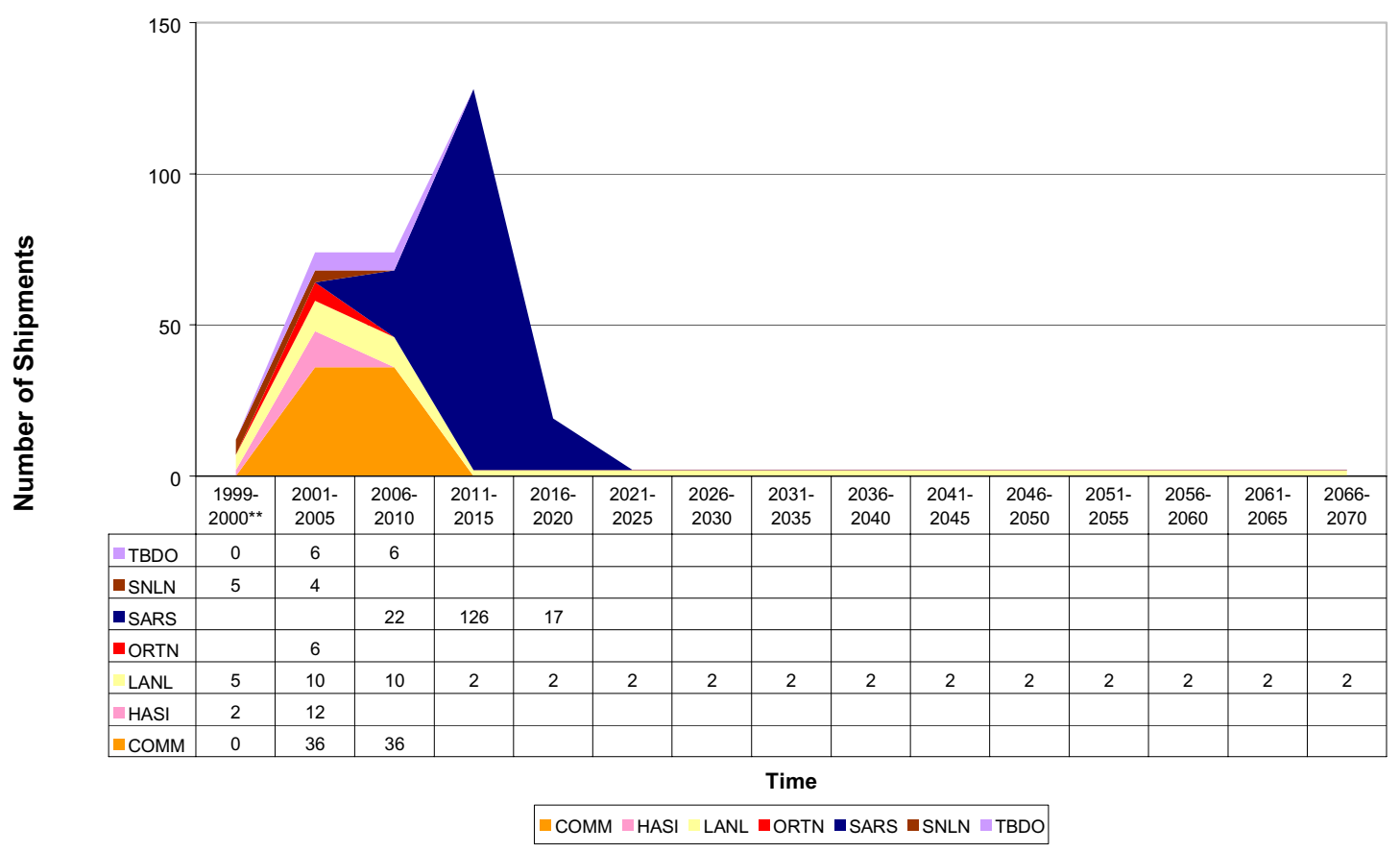

INEEL

Incoming LLW Shipments from "Commercial"

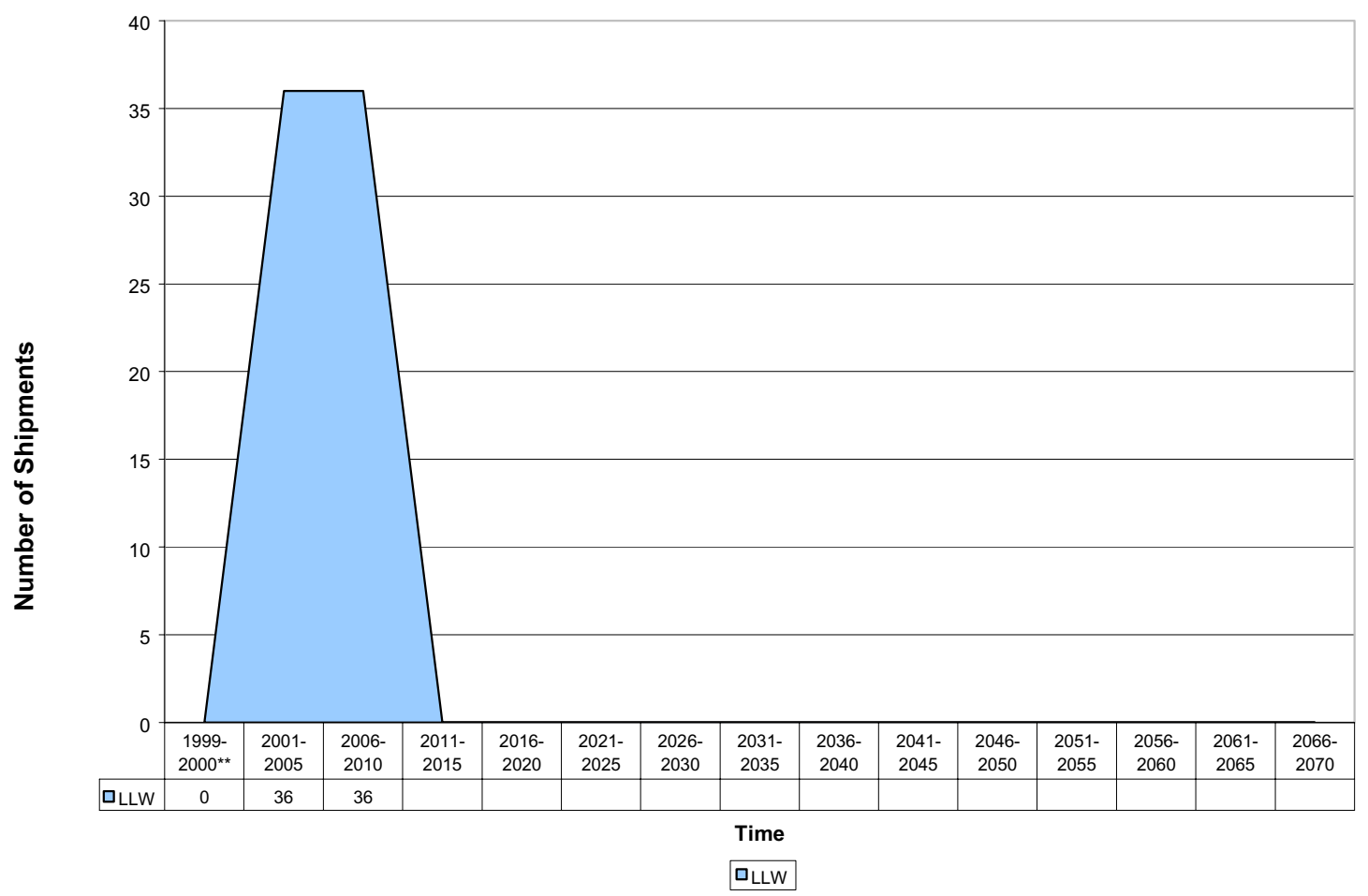

Total life-cycle values per 7/30/99 Stream Disposition Data (SDD) (http://id.inel.gov/avs/). 
INEEL

Incoming MLLW Shipments from Hanford

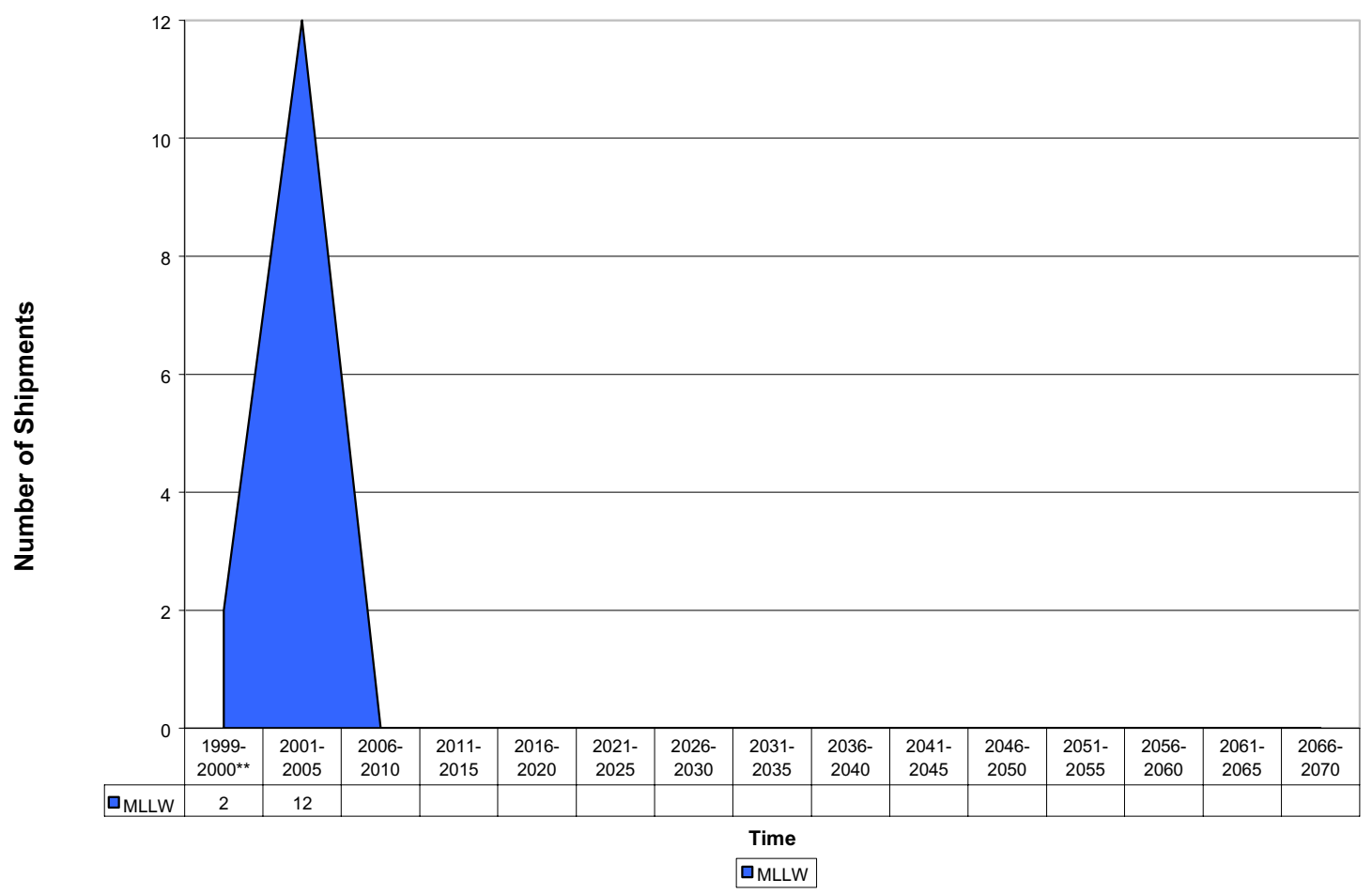

INEEL

Incoming MLLW Shipments from Sandia National Laboratory

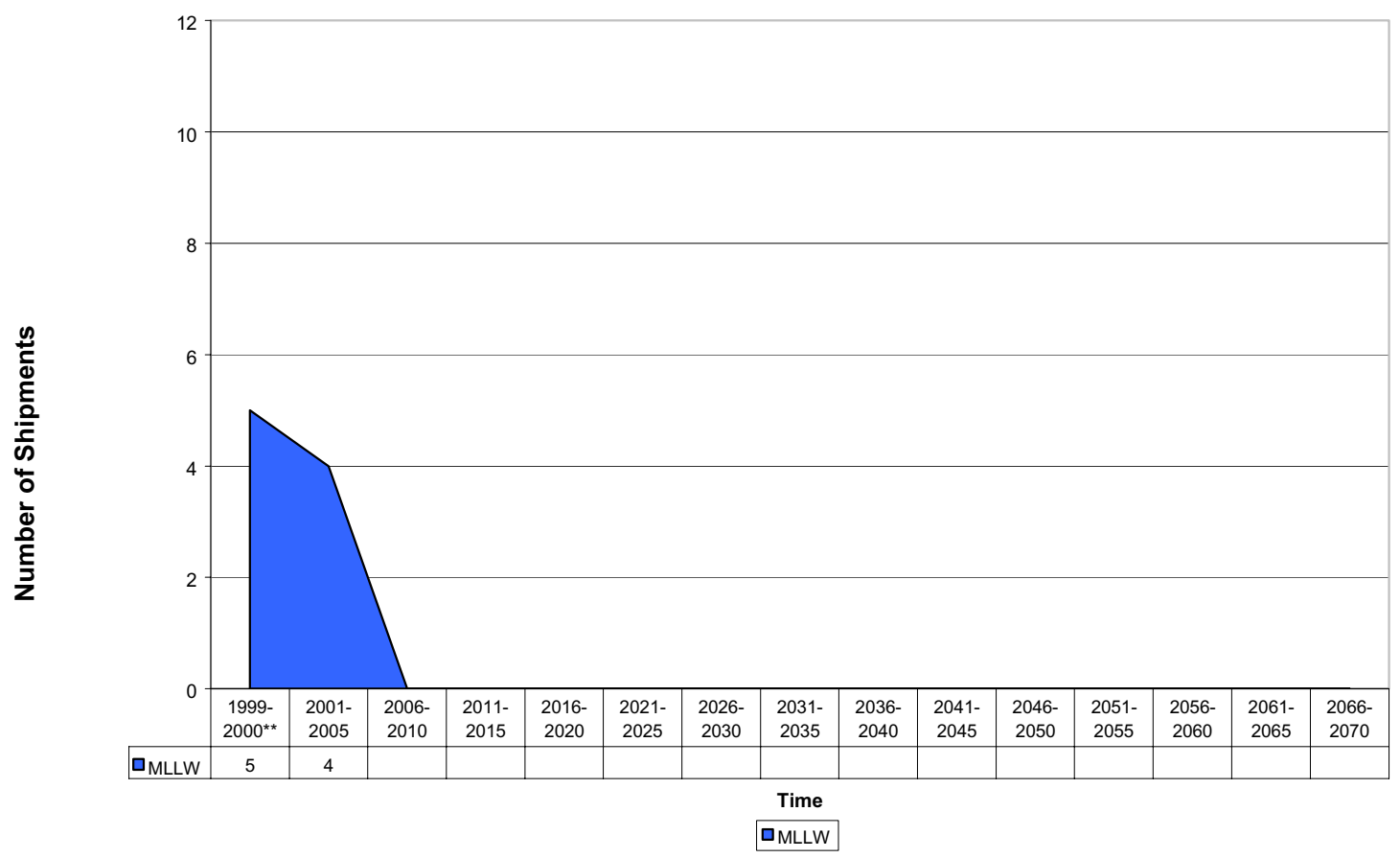

Total life-cycle values per 7/30/99 Stream Disposition Data (SDD) (http://id.inel.gov/avs/). 
INEEL

Incoming MLLW Shipments from

Los Alamos National Laboratory

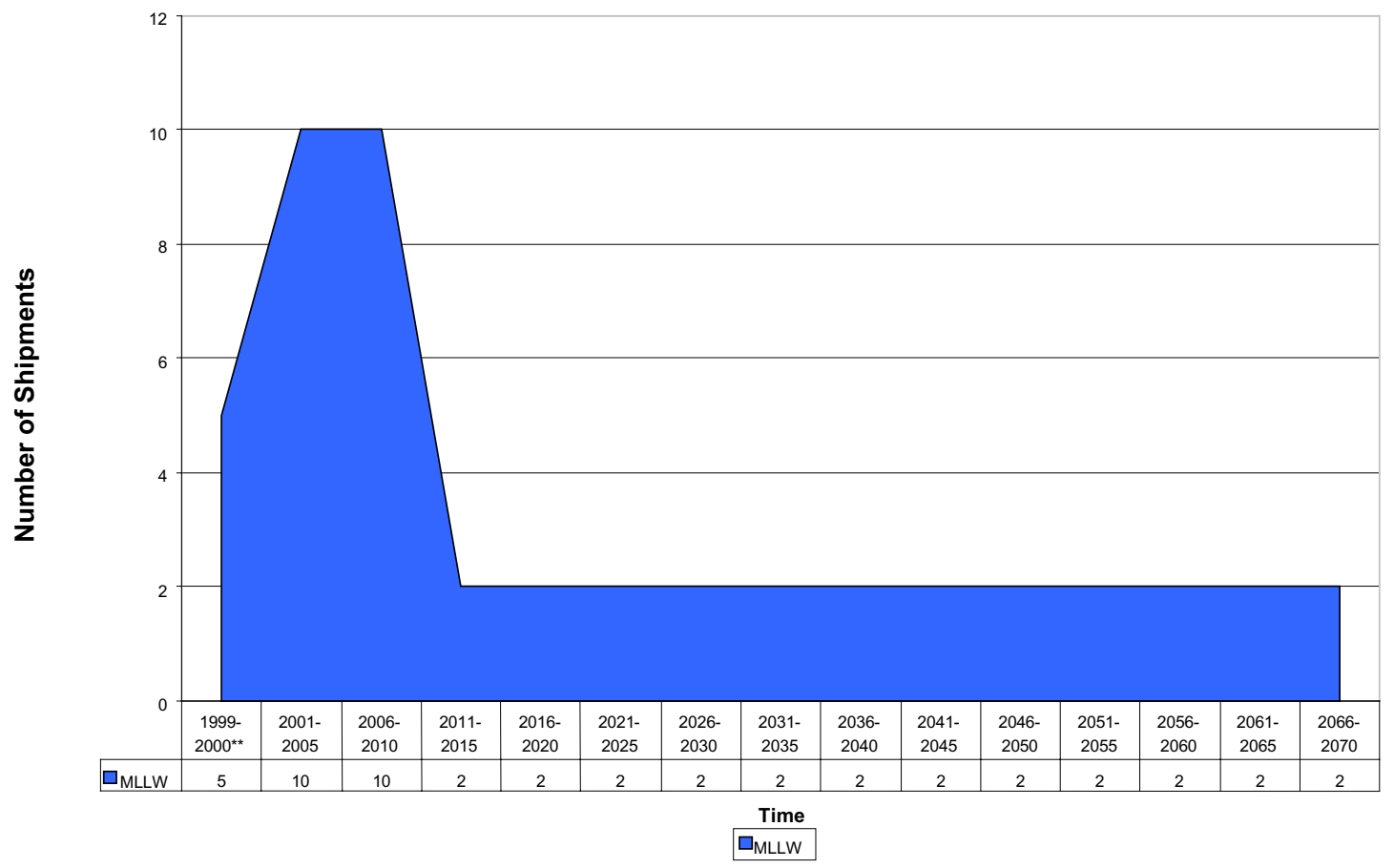

INEEL

Incoming SNF Shipments from

Savannah River Site

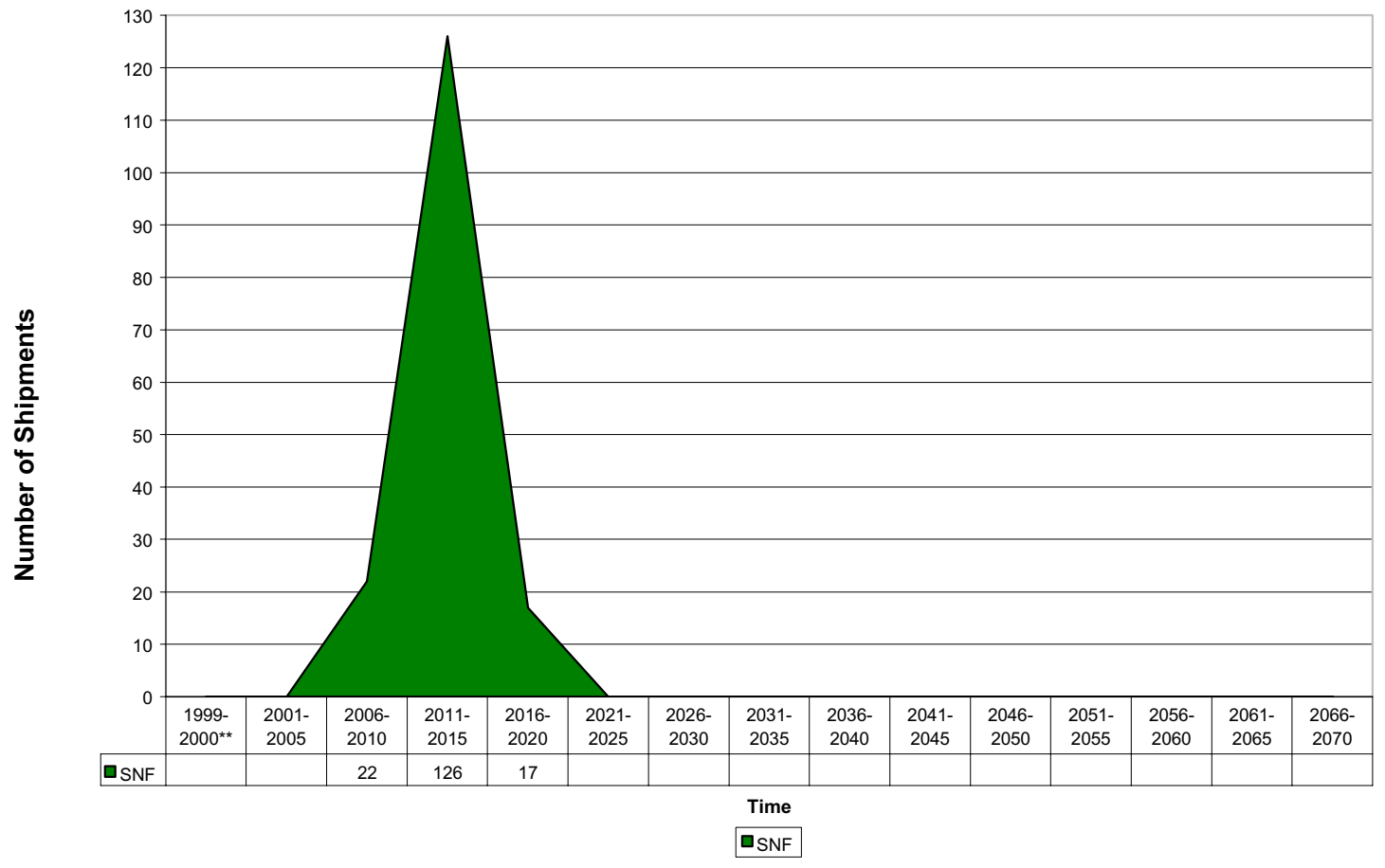

Total life-cycle values per 7/30/99 Stream Disposition Data (SDD) (http://id.inel.gov/avs/).

** Represents data for a two year period. 
INEEL

Incoming SNF Shipments from

Oak Ridge

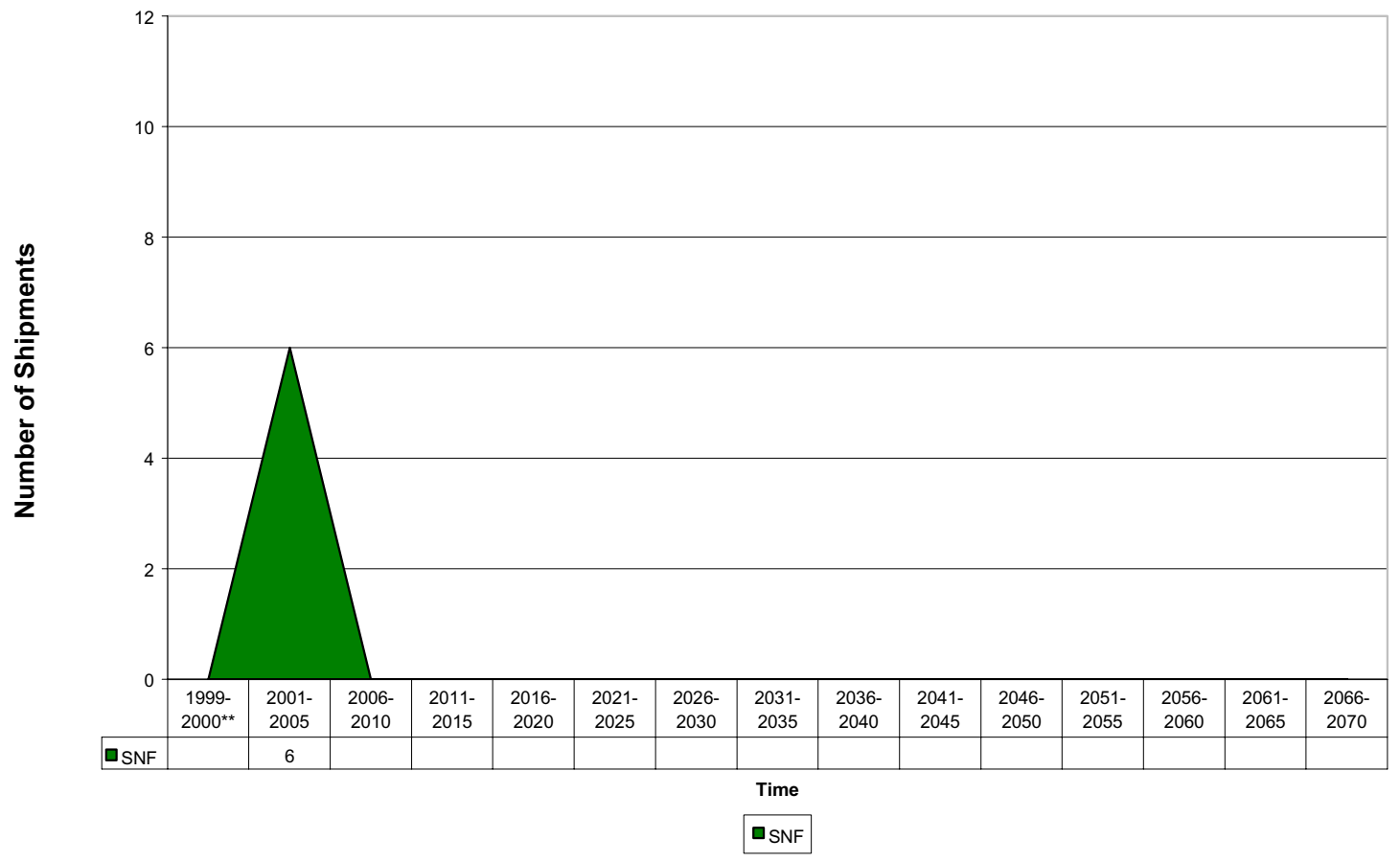

INEEL

Incoming MLLW Shipments from "TBDO"

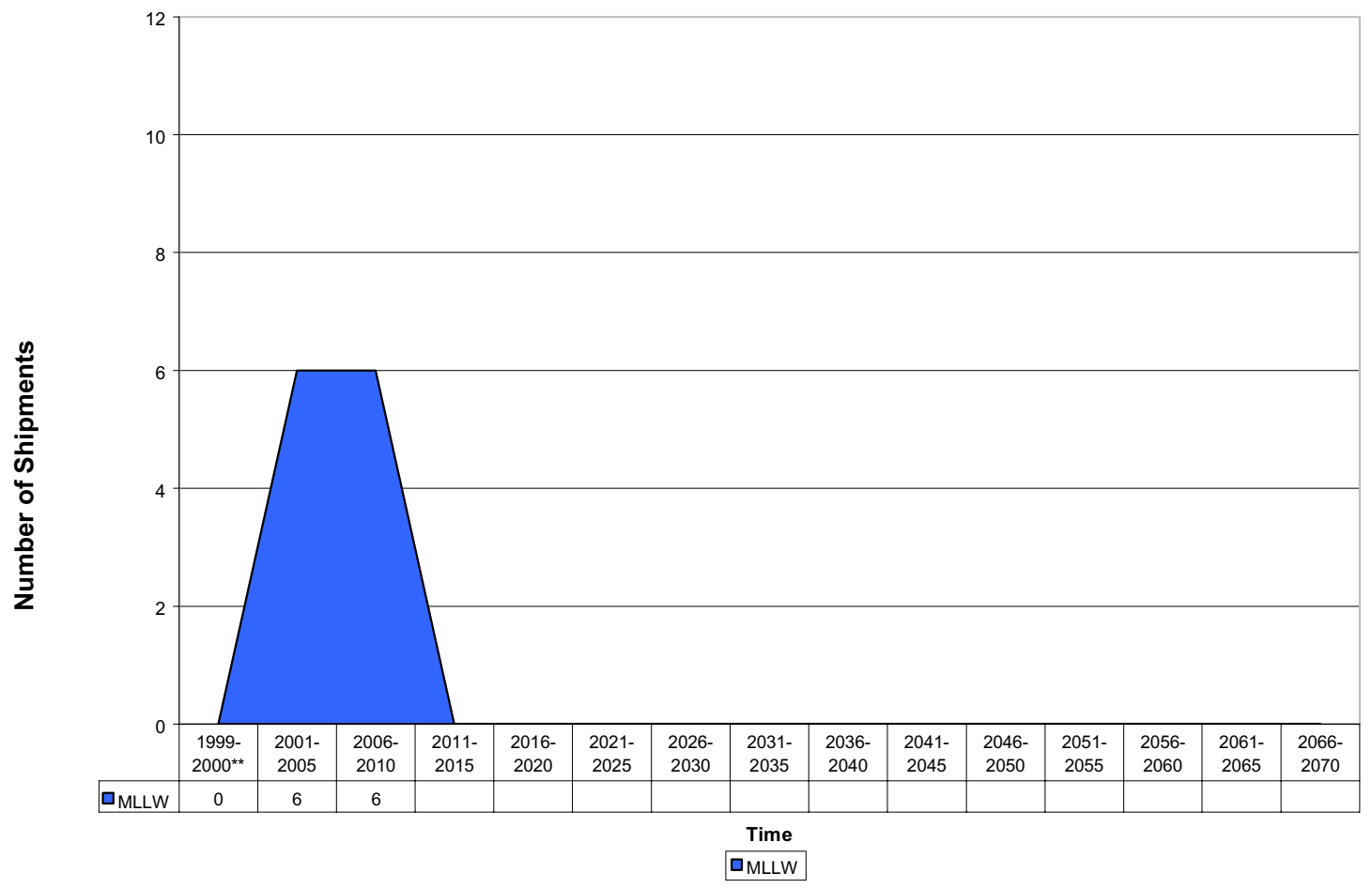

Total life-cycle values per 7/30/99 Stream Disposition Data (SDD) (http://id.inel.gov/avs/).

** Represents data for a two year period. 
INEEL

Waste Stream Disposition

Life-cycle Incoming Volume

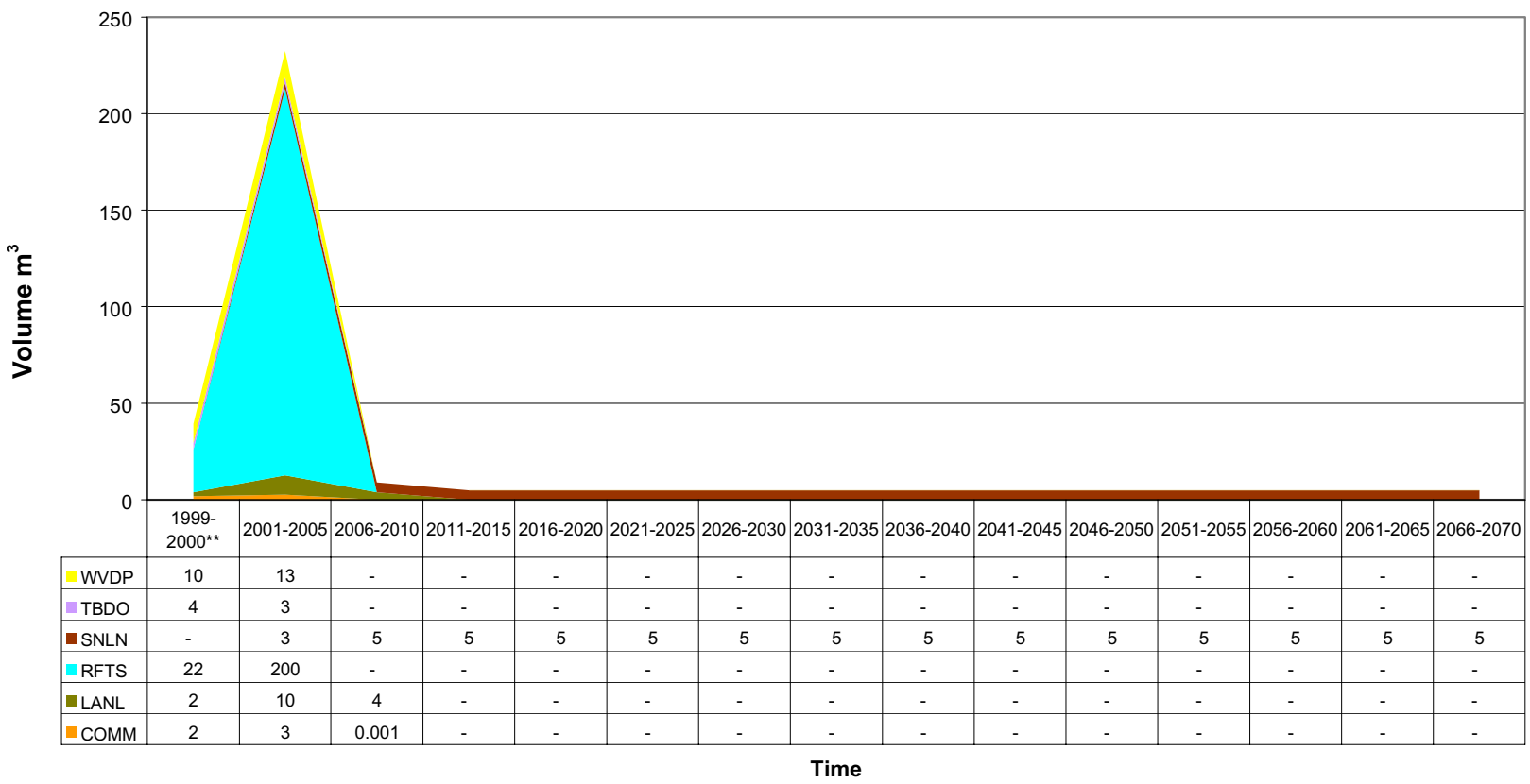

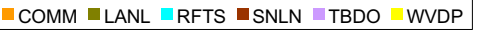

INEEL

Incoming M/LLW Volume from "Commercial"

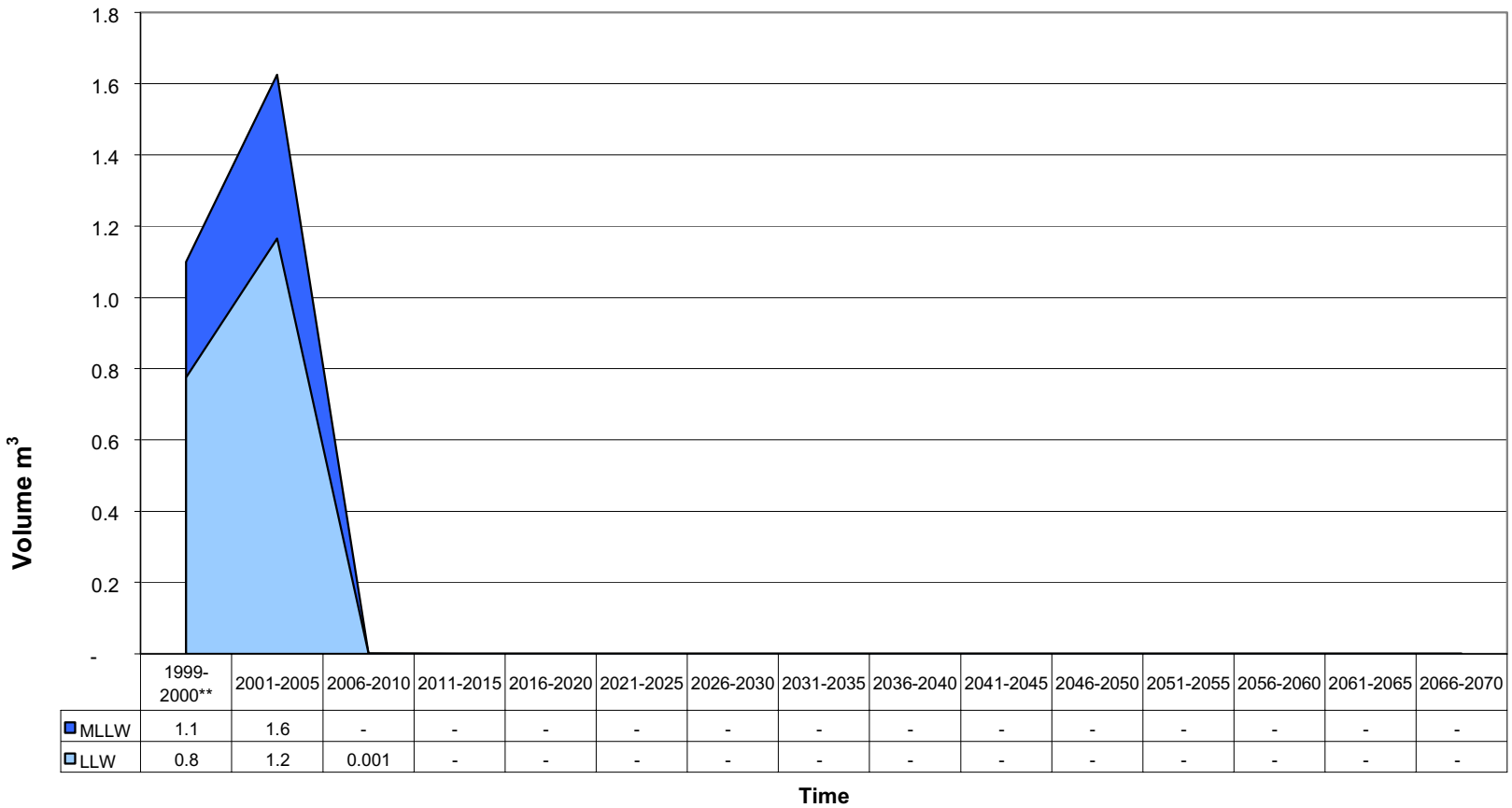

$\square_{\text {MLLW }} \mathbf{L L L W}_{\mathrm{LLW}}$

Total life-cycle values per 7/30/99 Stream Disposition Data (SDD) (http://id.inel.gov/avs/).

** Represents data for a two year period. 
INEEL

Incoming MLLW Volume from Los Alamos National Laboratory

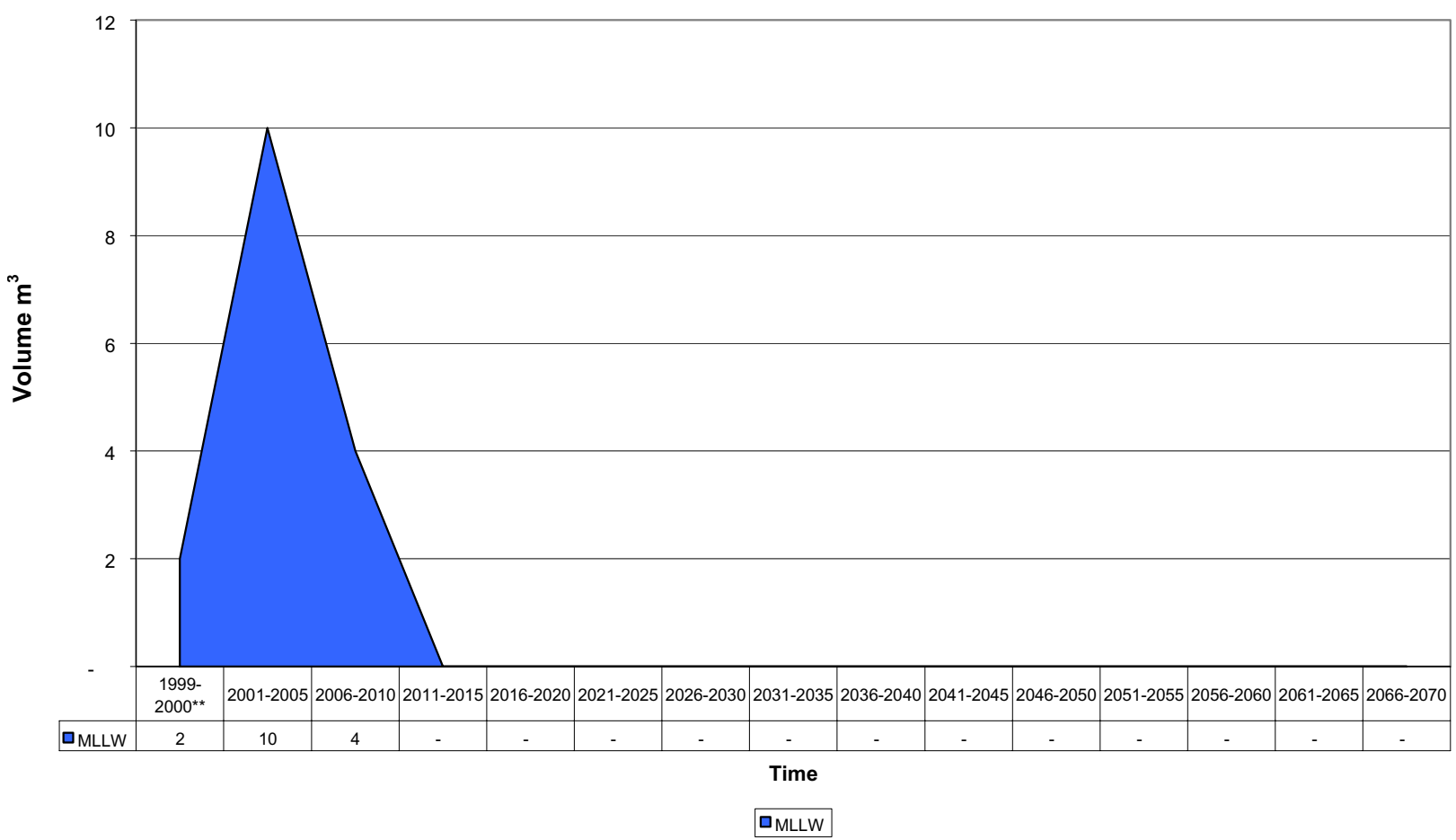

INEEL

Incoming MLLW \& TRU Volume from Rocky Flats

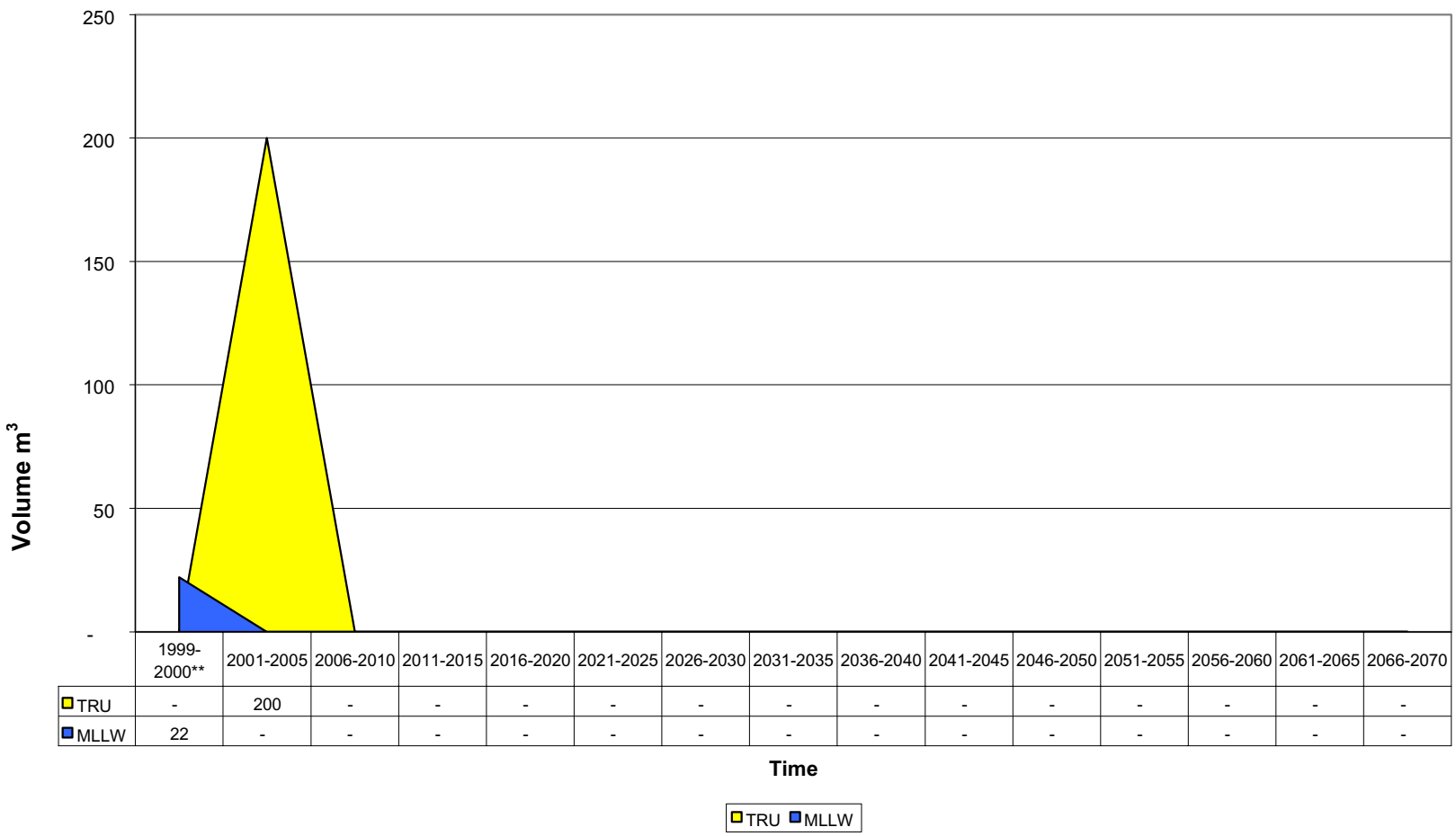

Total life-cycle values per 7/30/99 Stream Disposition Data (SDD) (http://id.inel.gov/avs/).

** Represents data for a two year period. 
INEEL

Incoming MLLW Volume from Sandia Naitonal Labatory

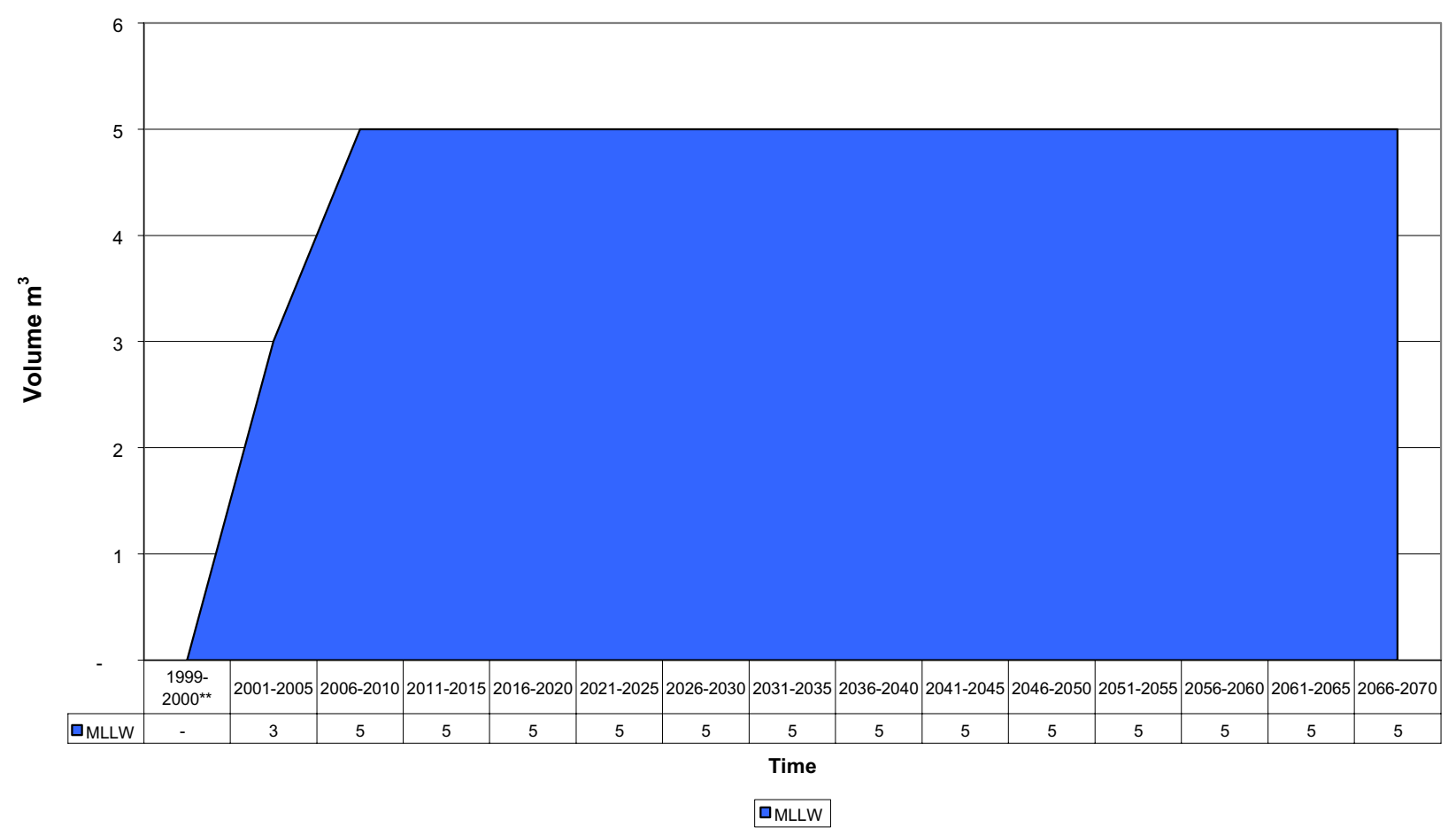

INEEL

Incoming MLLW Volume from "TBDO"

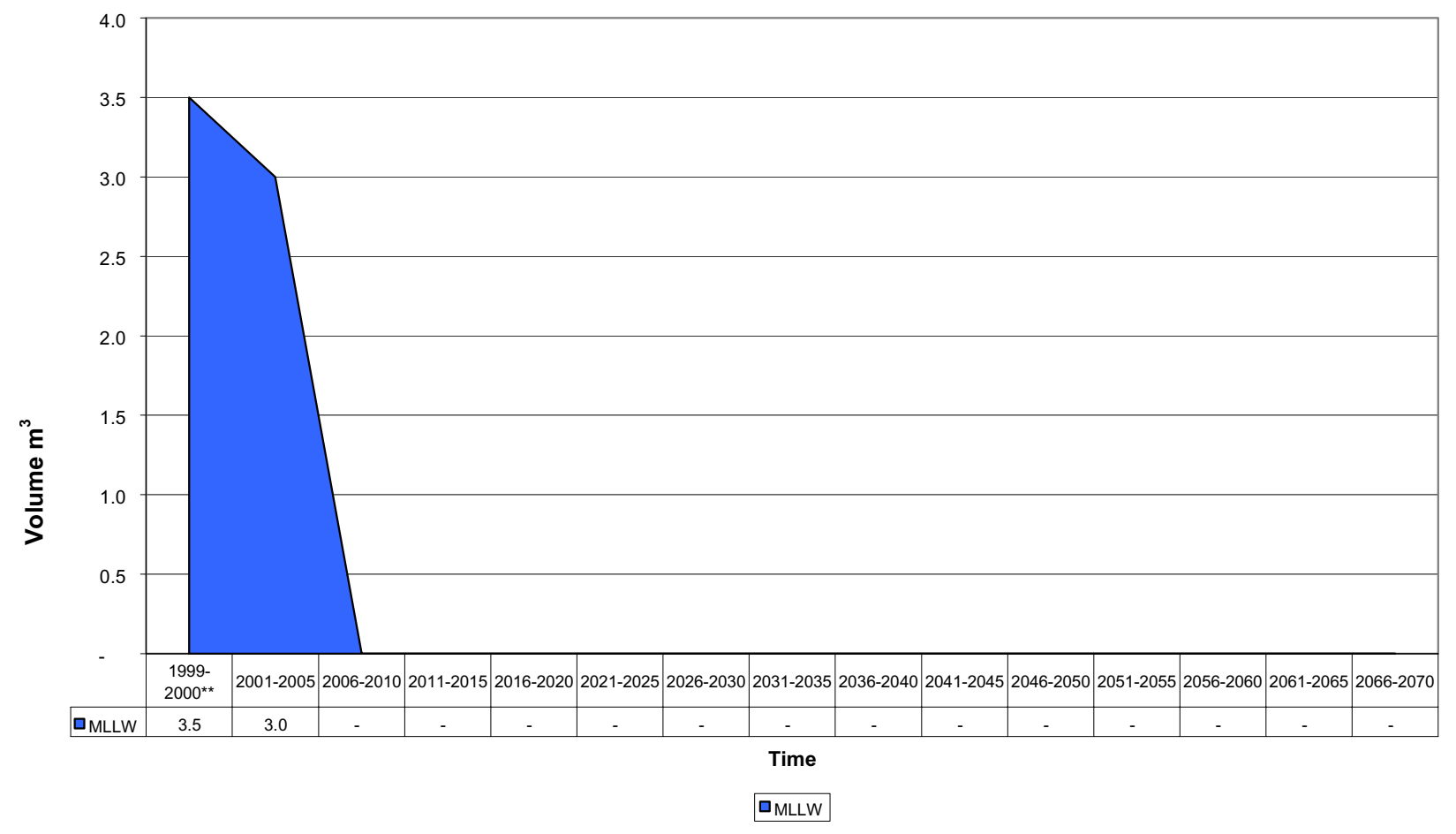

Total life-cycle values per 7/30/99 Stream Disposition Data (SDD) (http://id.inel.gov/avs/). 
INEEL

Incoming MLLW Volume from West Valley

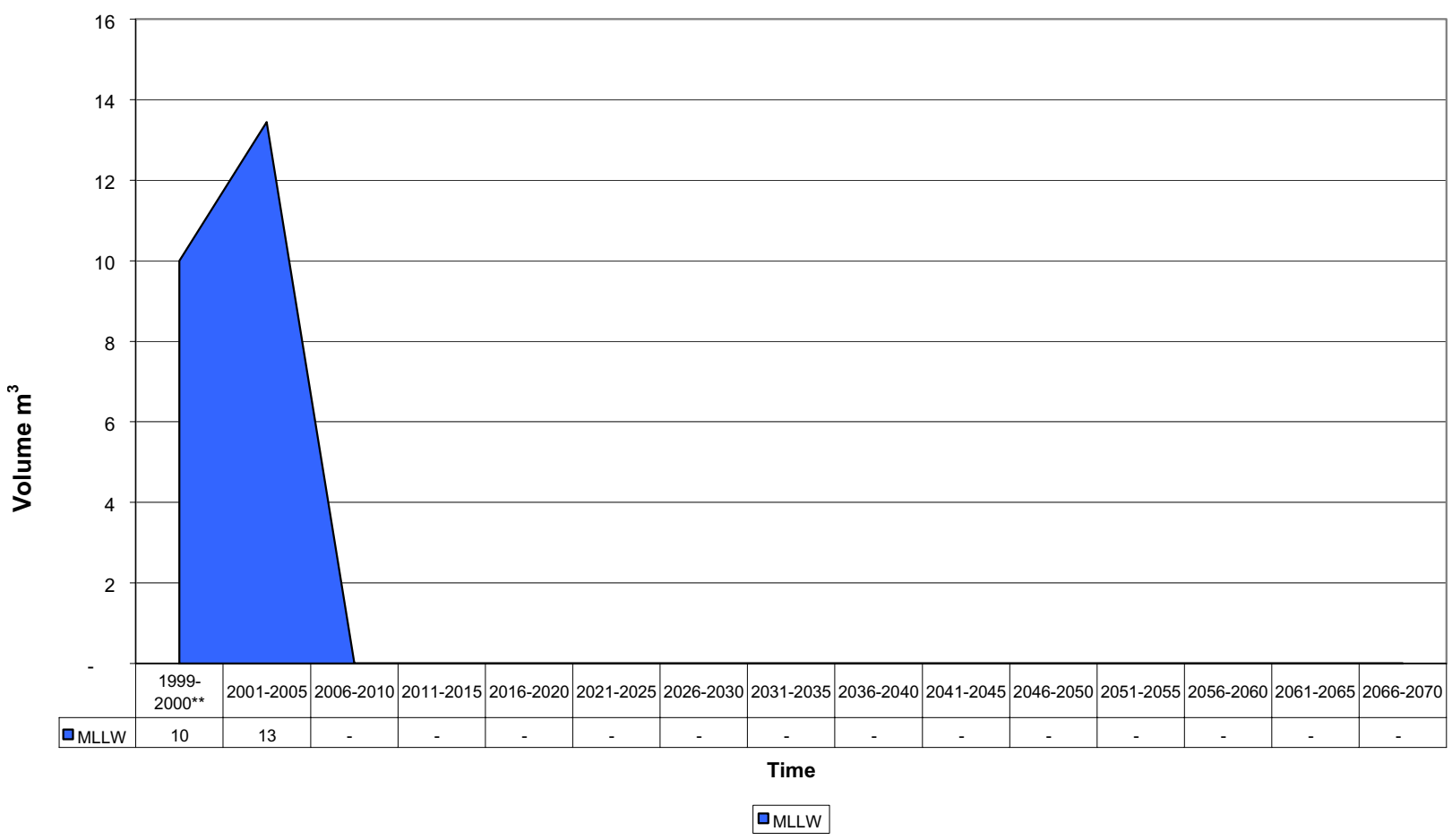

INEEL

Waste Stream Disposition

Life-cycle Incoming SNF

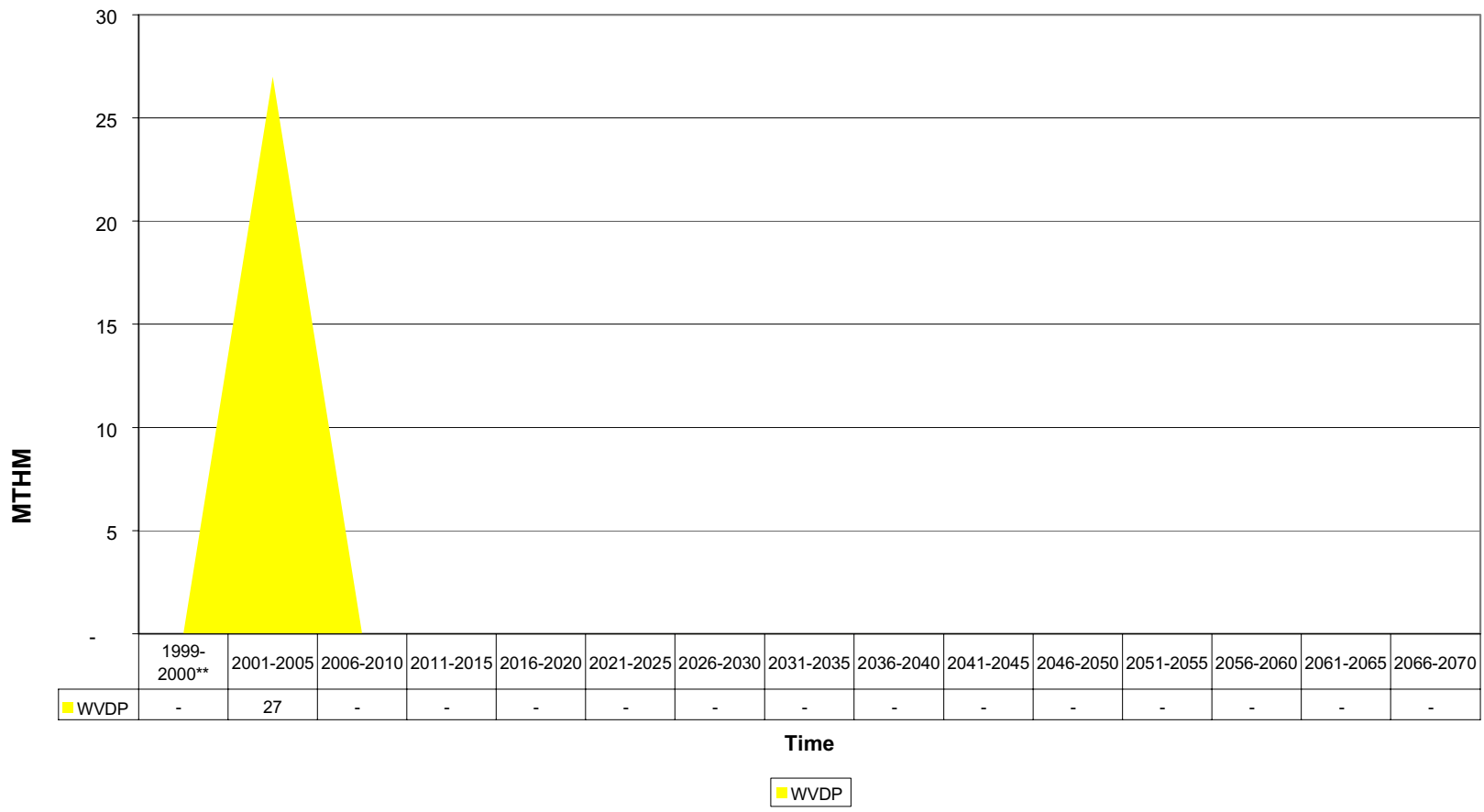

Total life-cycle values per 7/30/99 Stream Disposition Data (SDD) (http://id.inel.gov/avs/).

** Represents data for a two year period. 
INEEL

Incoming SNF from West Valley

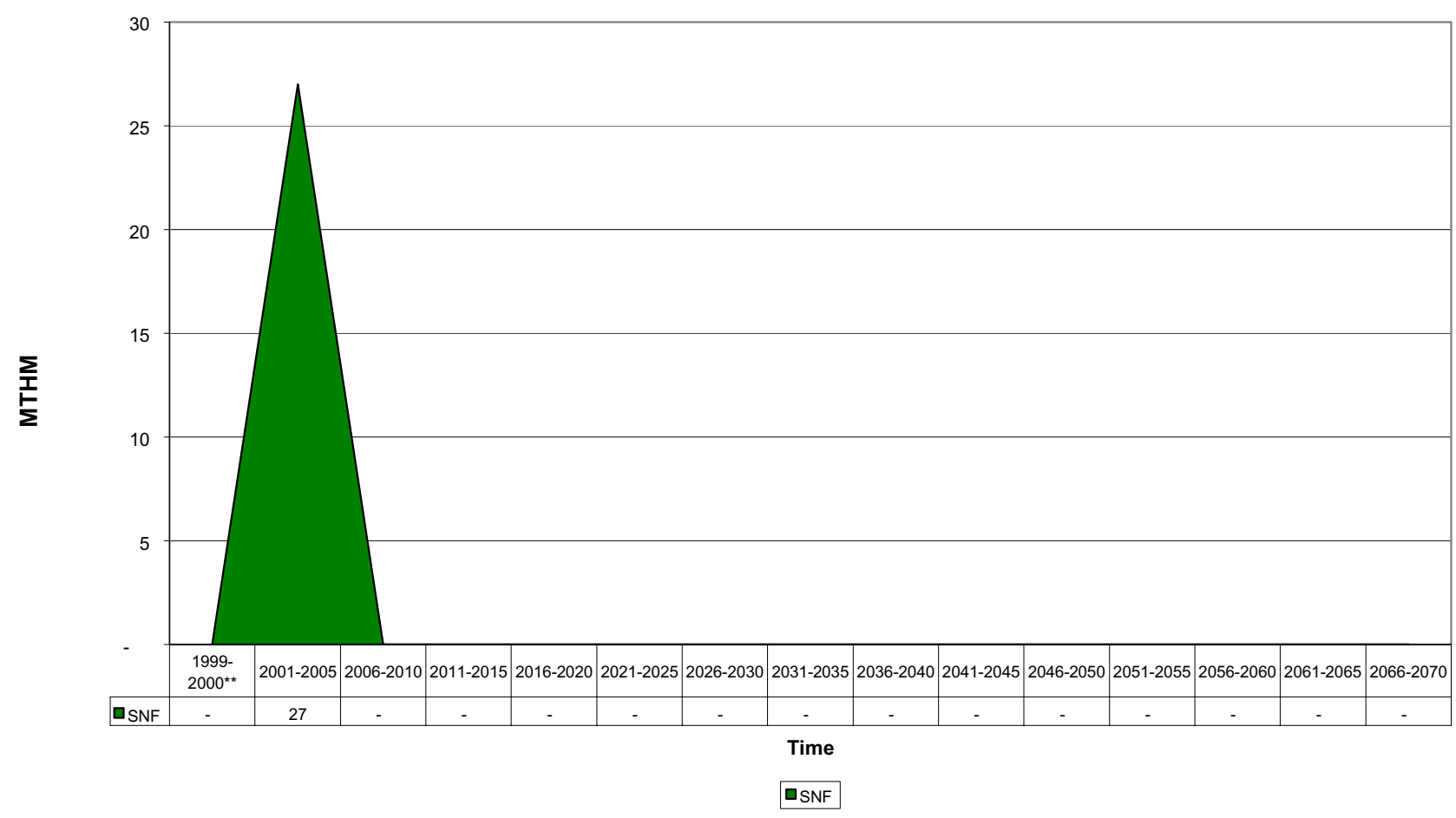

Total life-cycle values per 7/30/99 Stream Disposition Data (SDD) (http://id.inel.gov/avs/).

** Represents data for a two year period. 


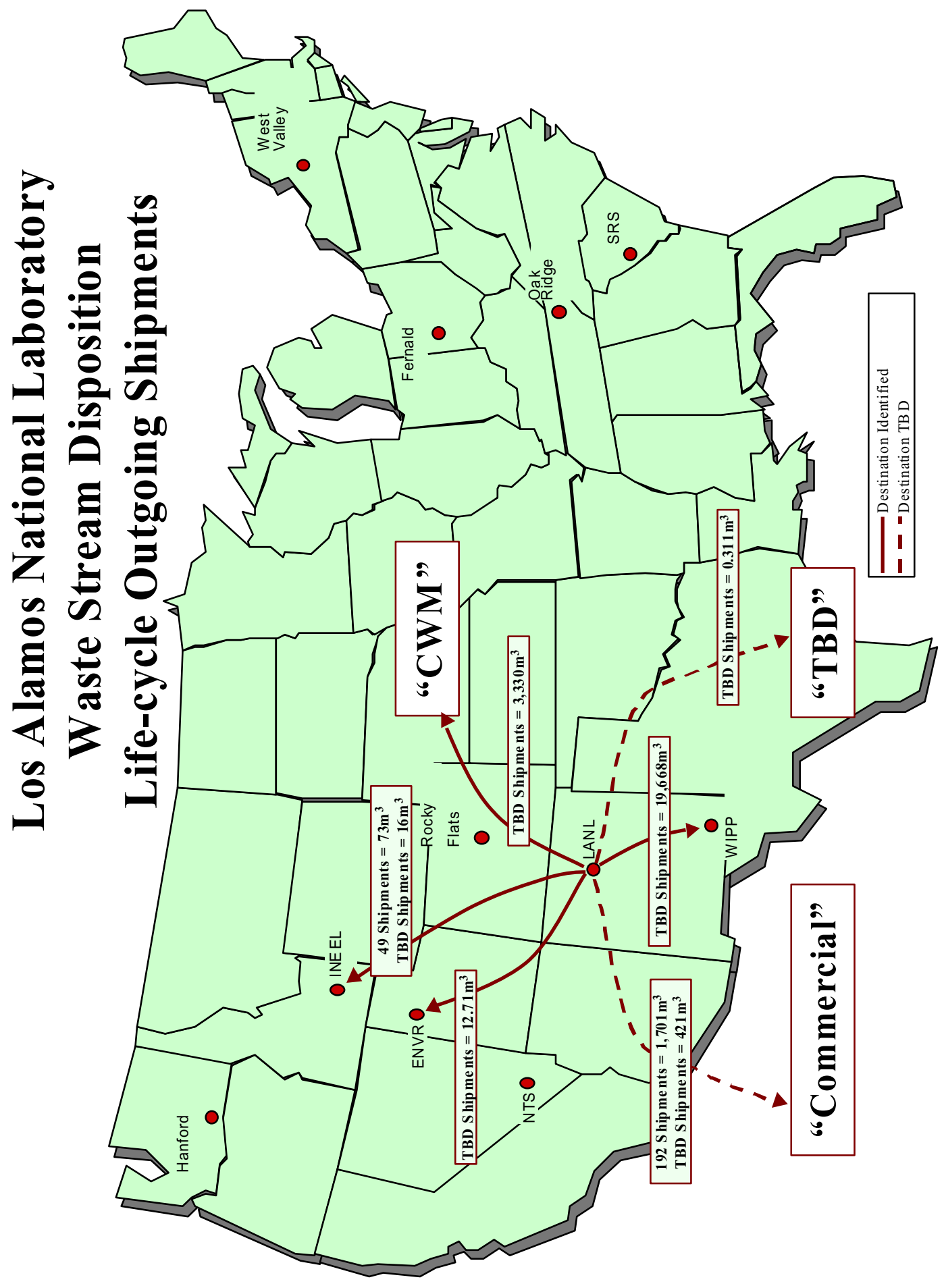




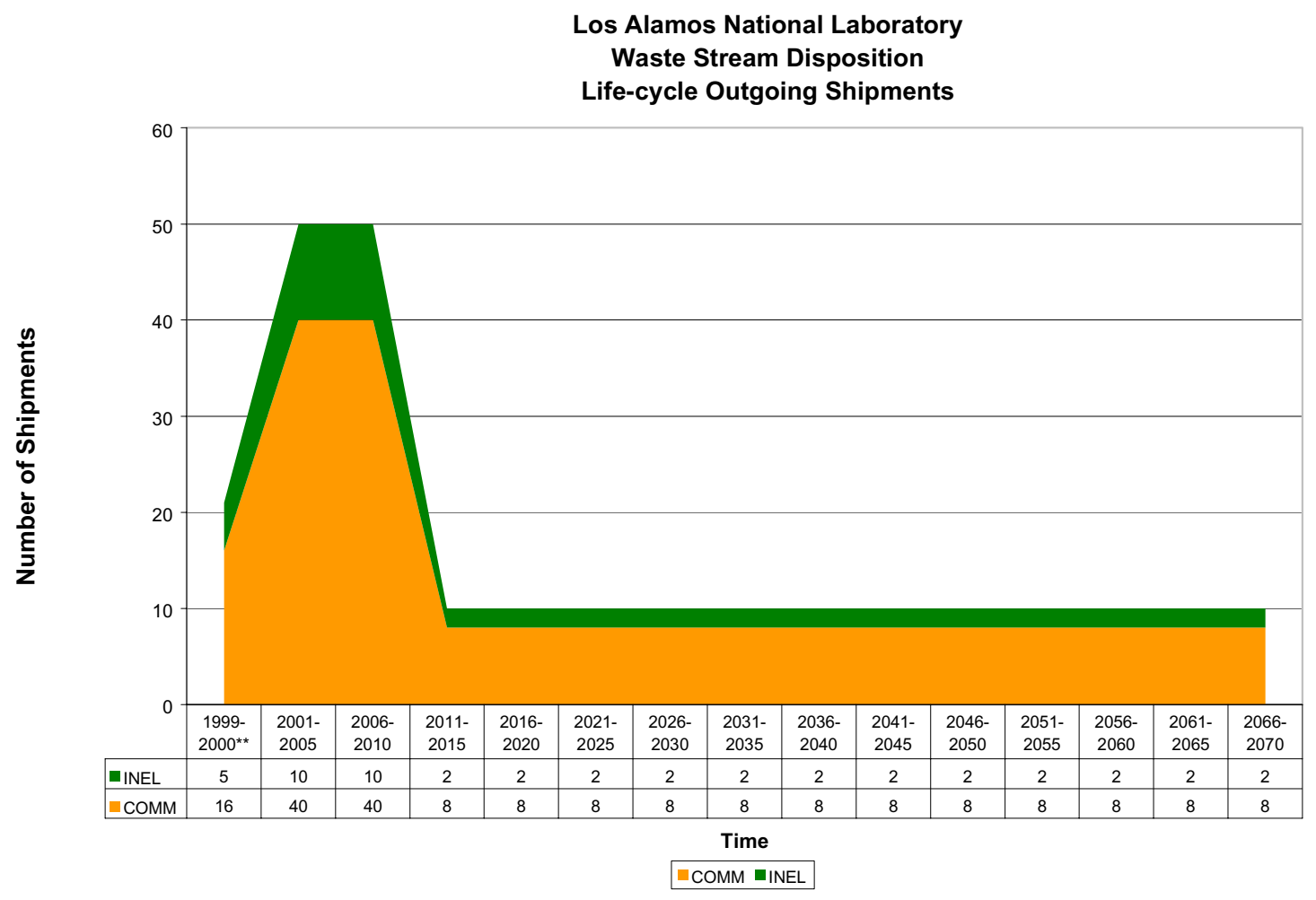

Los Alamos National Laboratory

Outgoing MLLW Shipments to INEEL

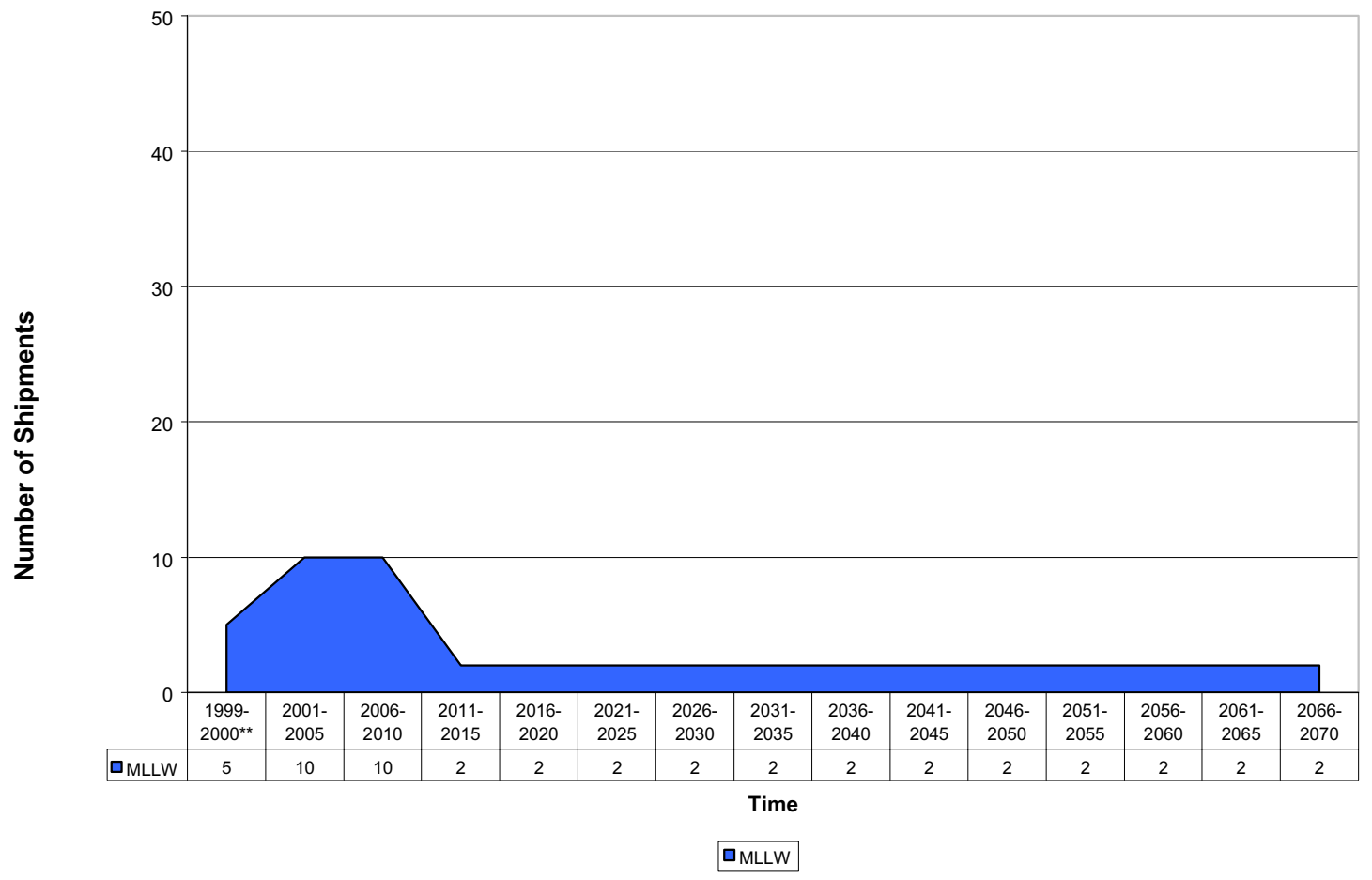

Total life-cycle values per 7/30/99 Stream Disposition Data (SDD) (http://id.inel.gov/avs/).

** Represents data for a two year period. 
Los Alamos National Laboratory

Outgoing MLLW Shipments to "Commercial"

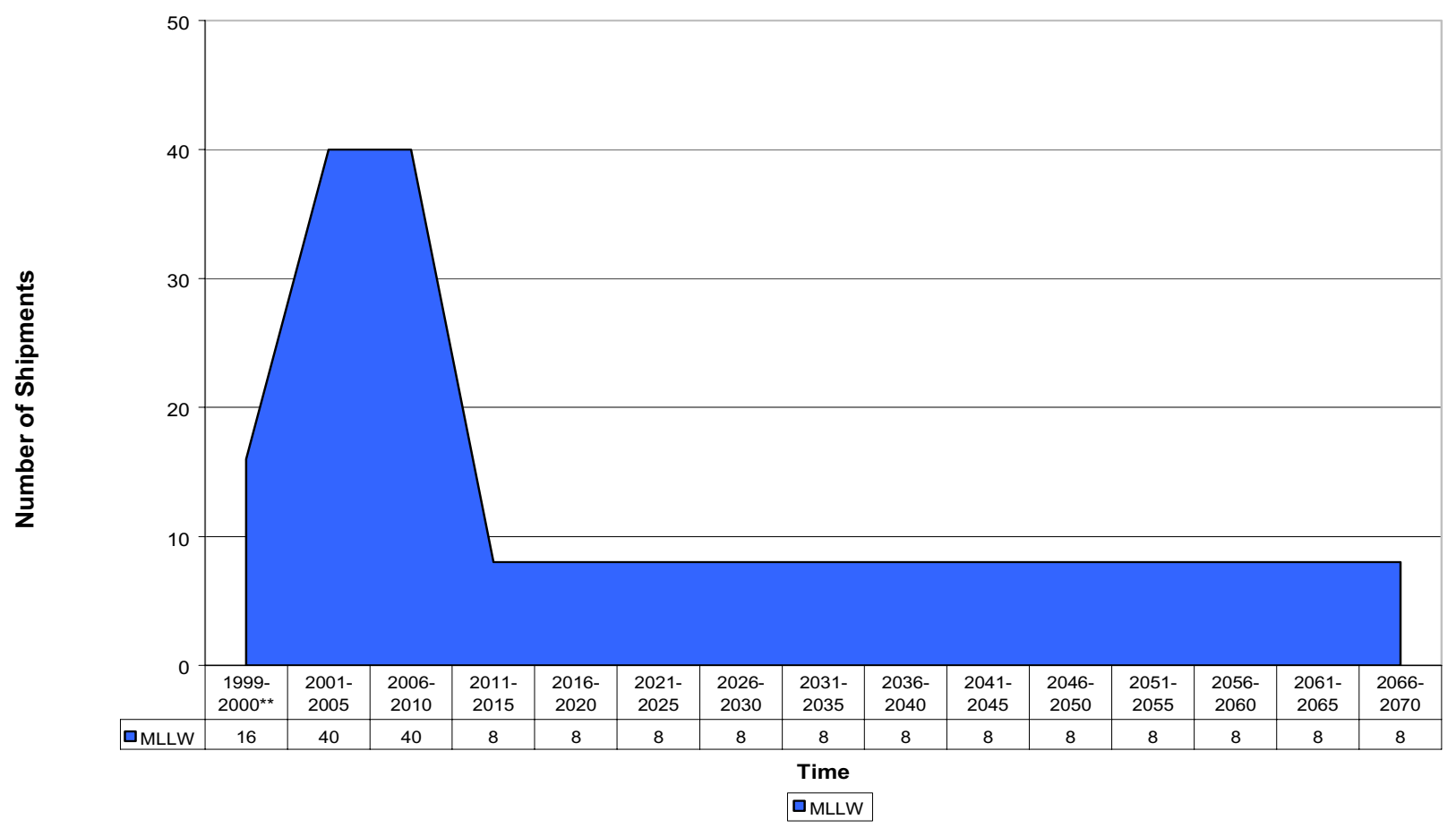

Los Alamos National Laboratory

Waste Stream Disposition

Life-cycle Outgoing Volume

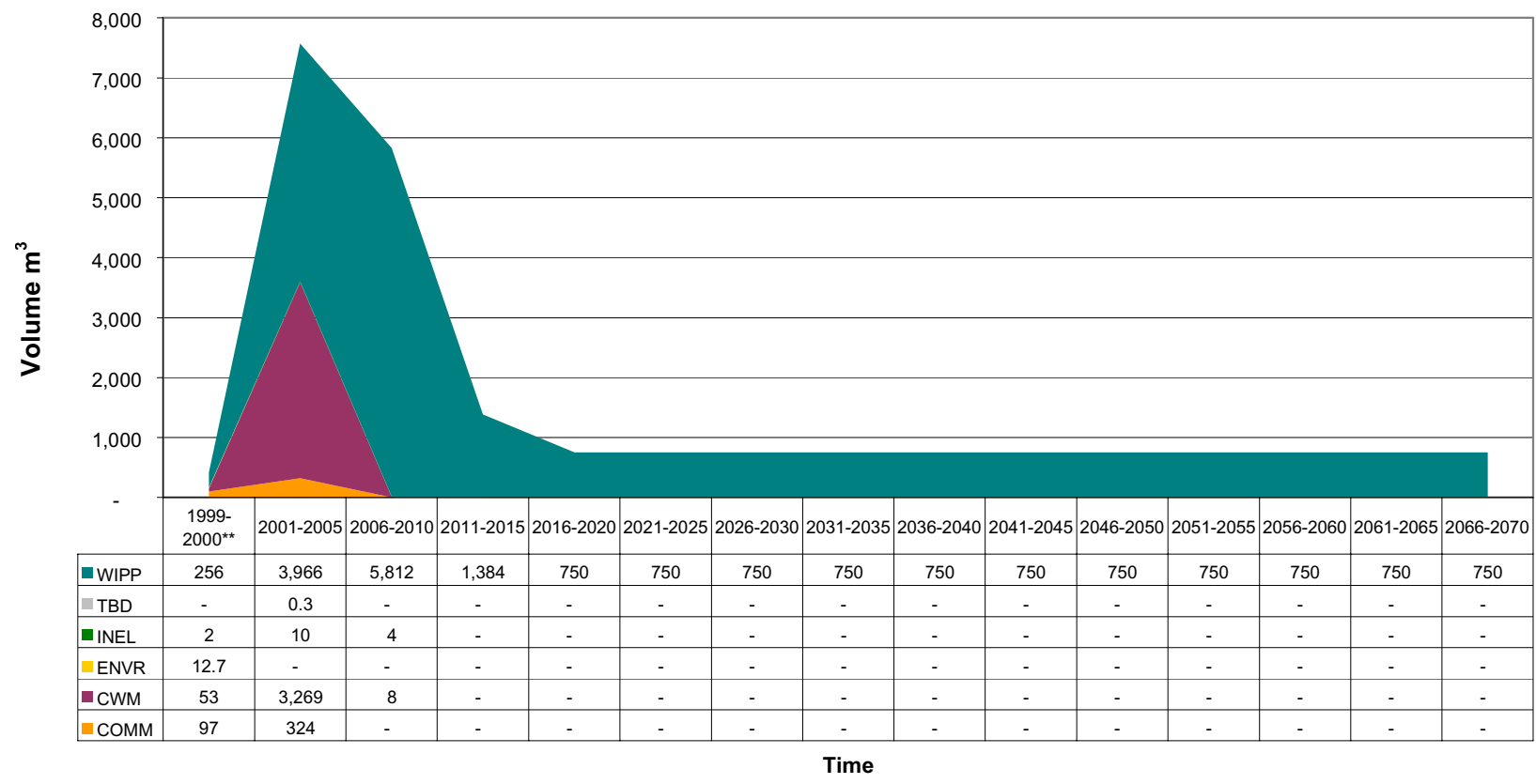

$\square$ COMM $\square$ CWM $\square$ ENVR $\square$ INEL $\square$ TBD $\square$ WIPP

Total life-cycle values per 7/30/99 Stream Disposition Data (SDD) (http://id.inel.gov/avs/).

** Represents data for a two year period. 
Los Alamos National Laboratory

Outgoing MLLW Volume to "Commercial"

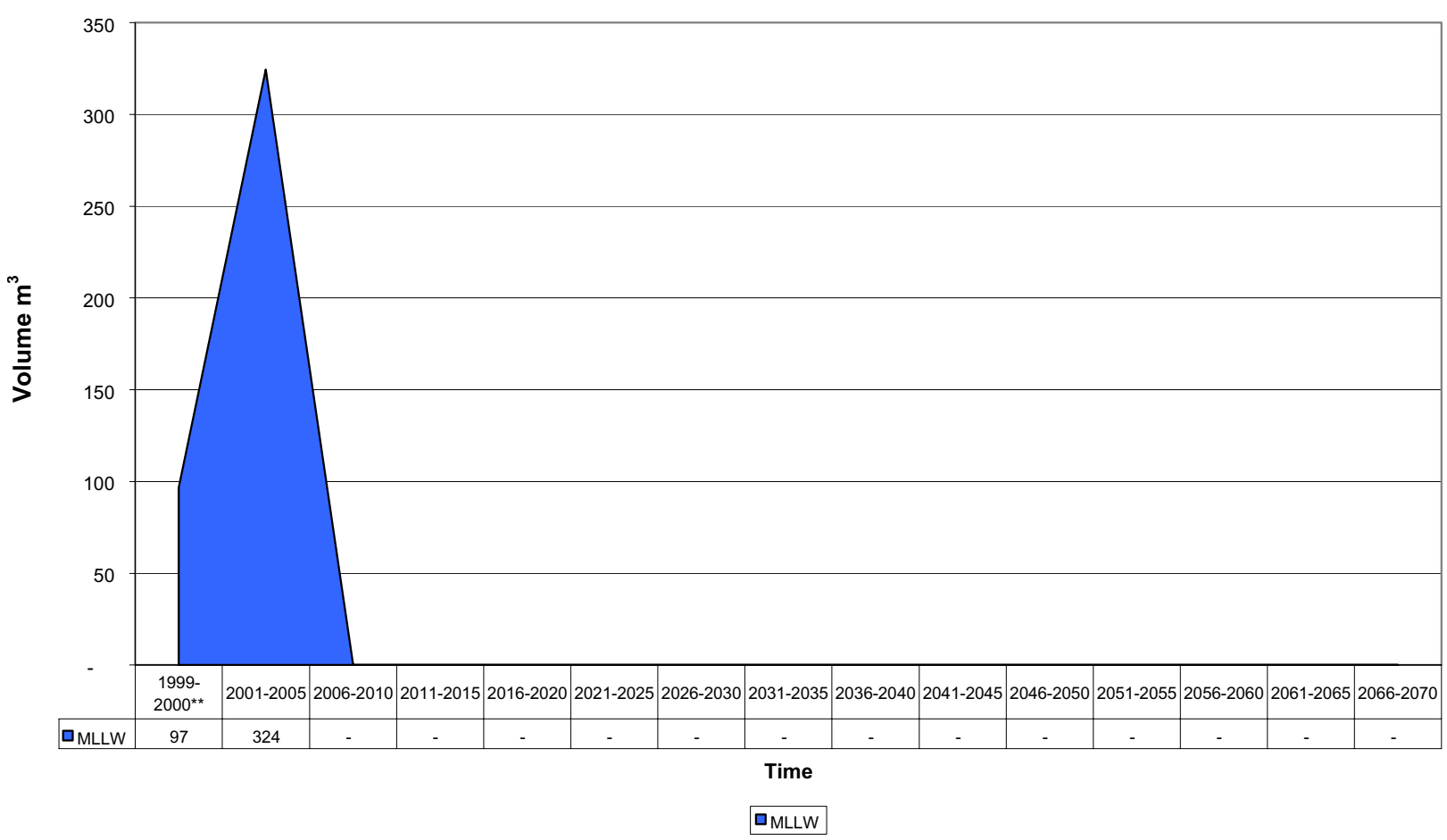

Los Alamos National Laboratory

Outgoing MLLW Volume to Chemical Waste Management

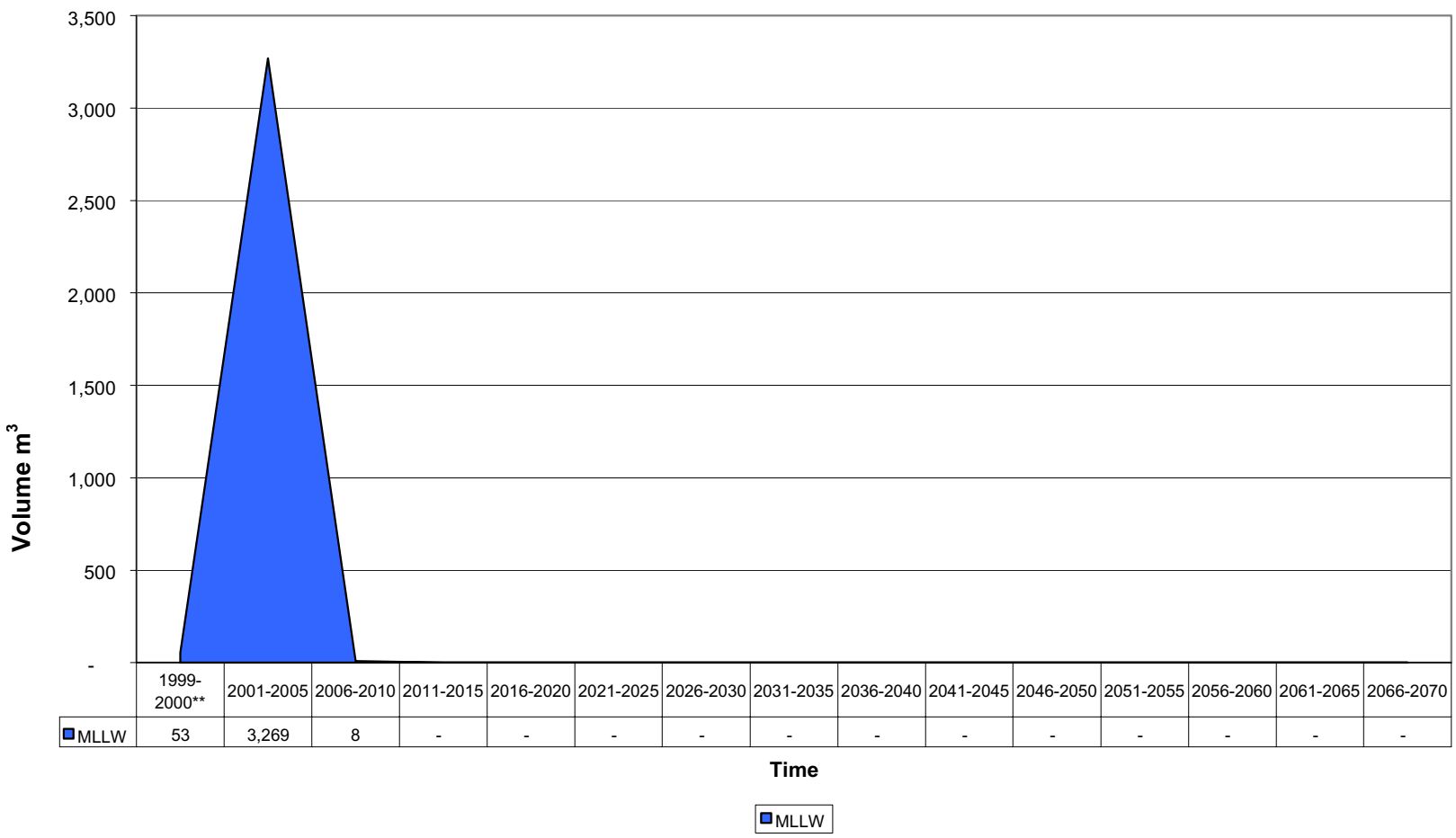

Total life-cycle values per 7/30/99 Stream Disposition Data (SDD) (http://id.inel.gov/avs/).

** Represents data for a two year period. 


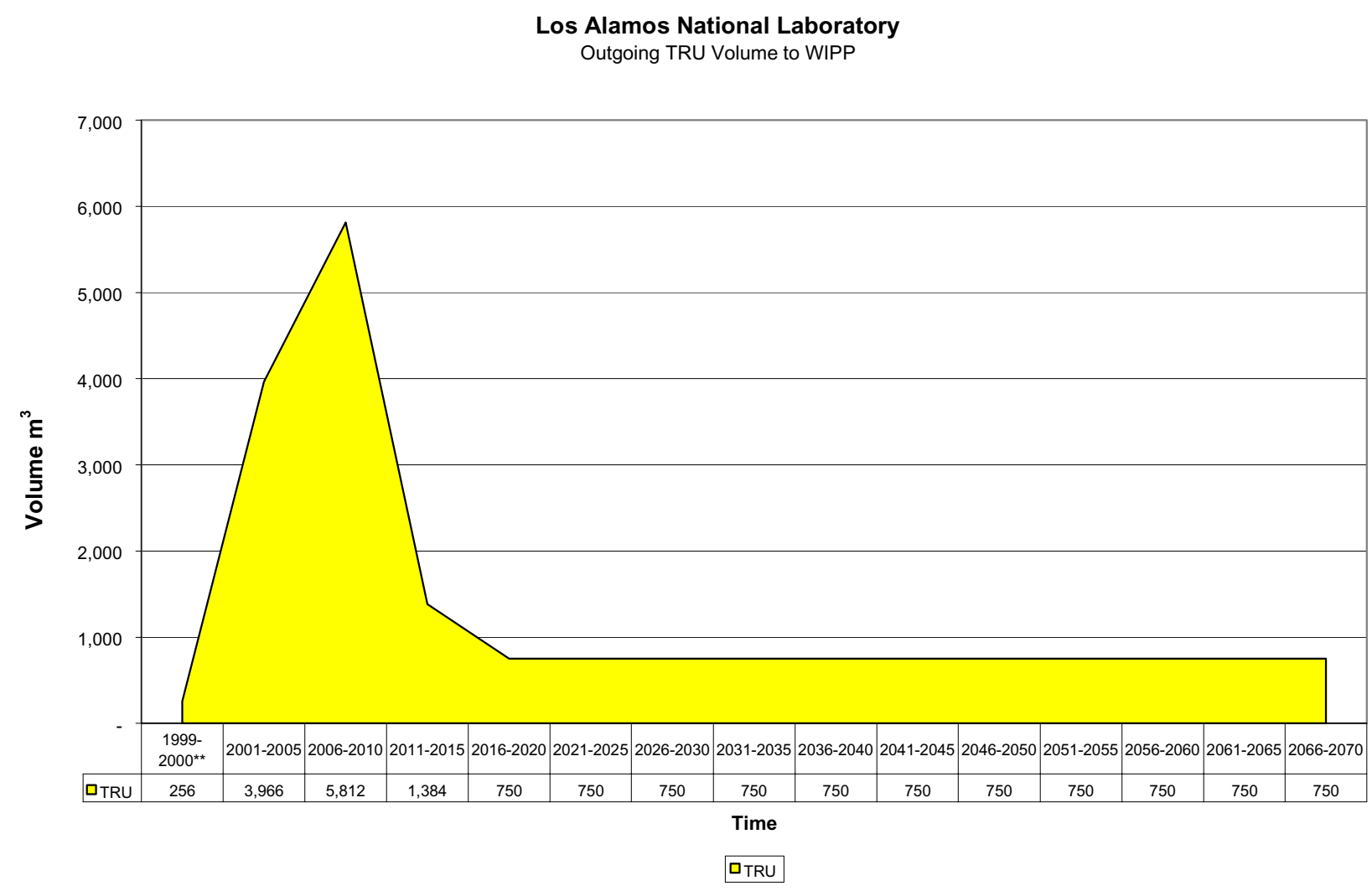

Los Alamos National Laboratory

Outgoing MLLW Volume to Envirocare

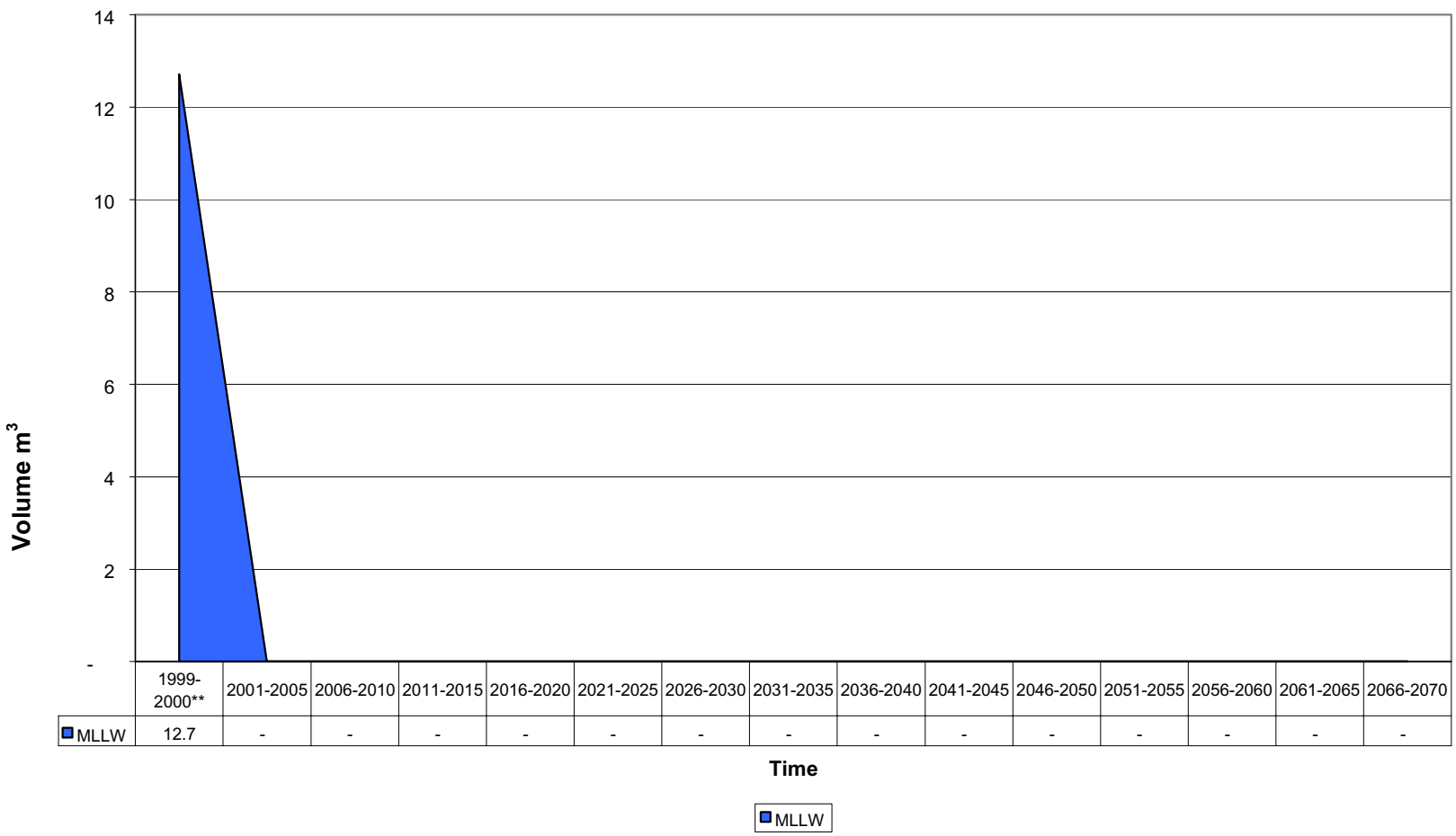

Total life-cycle values per 7/30/99 Stream Disposition Data (SDD) (http://id.inel.gov/avs/).

** Represents data for a two year period. 
Los Alamos National Laboratory

Outgoing MLLW Volume to INEEL

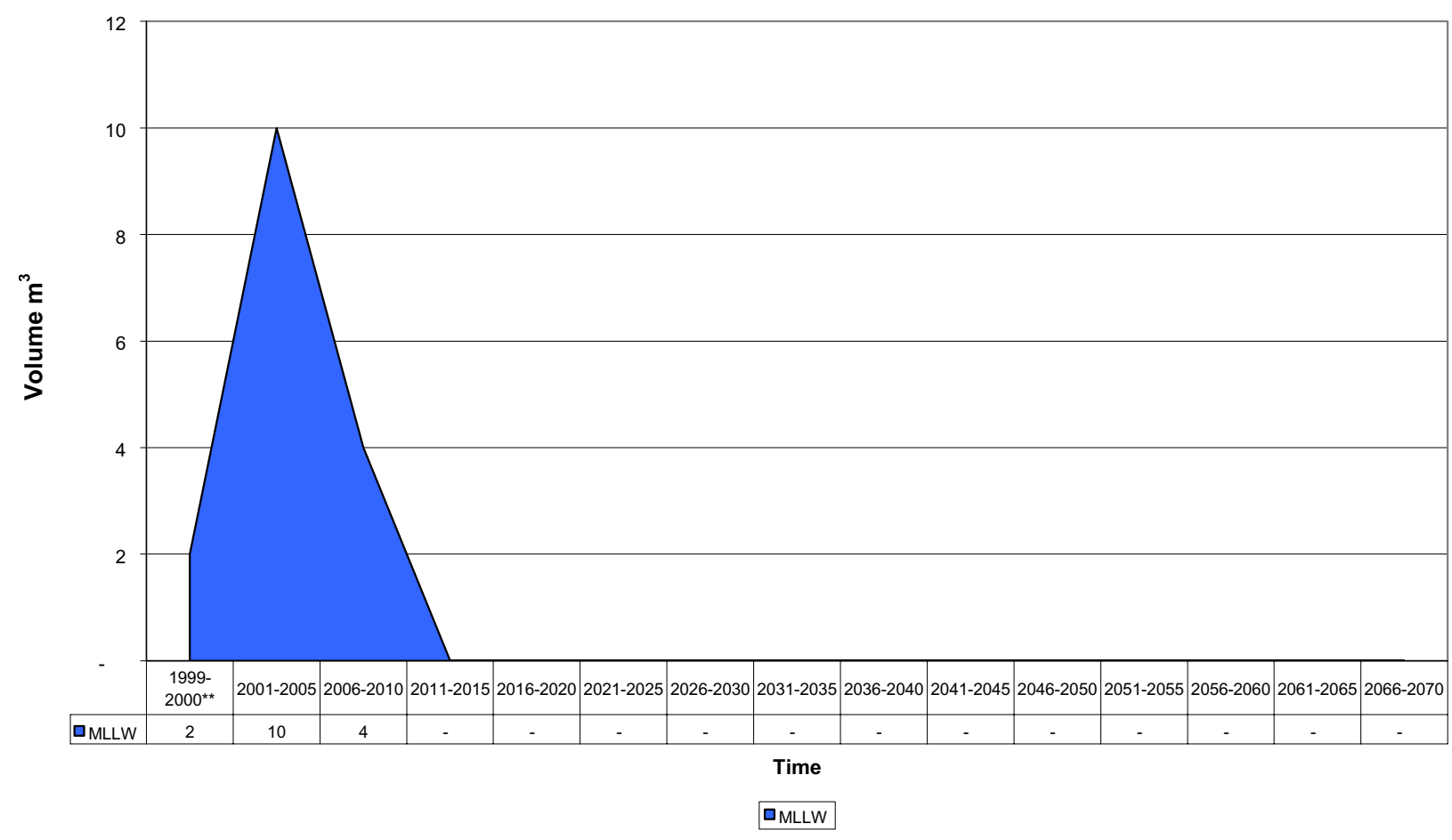

Los Alamos National Laboratory

Outgoing MLLW Volume to "TBD"

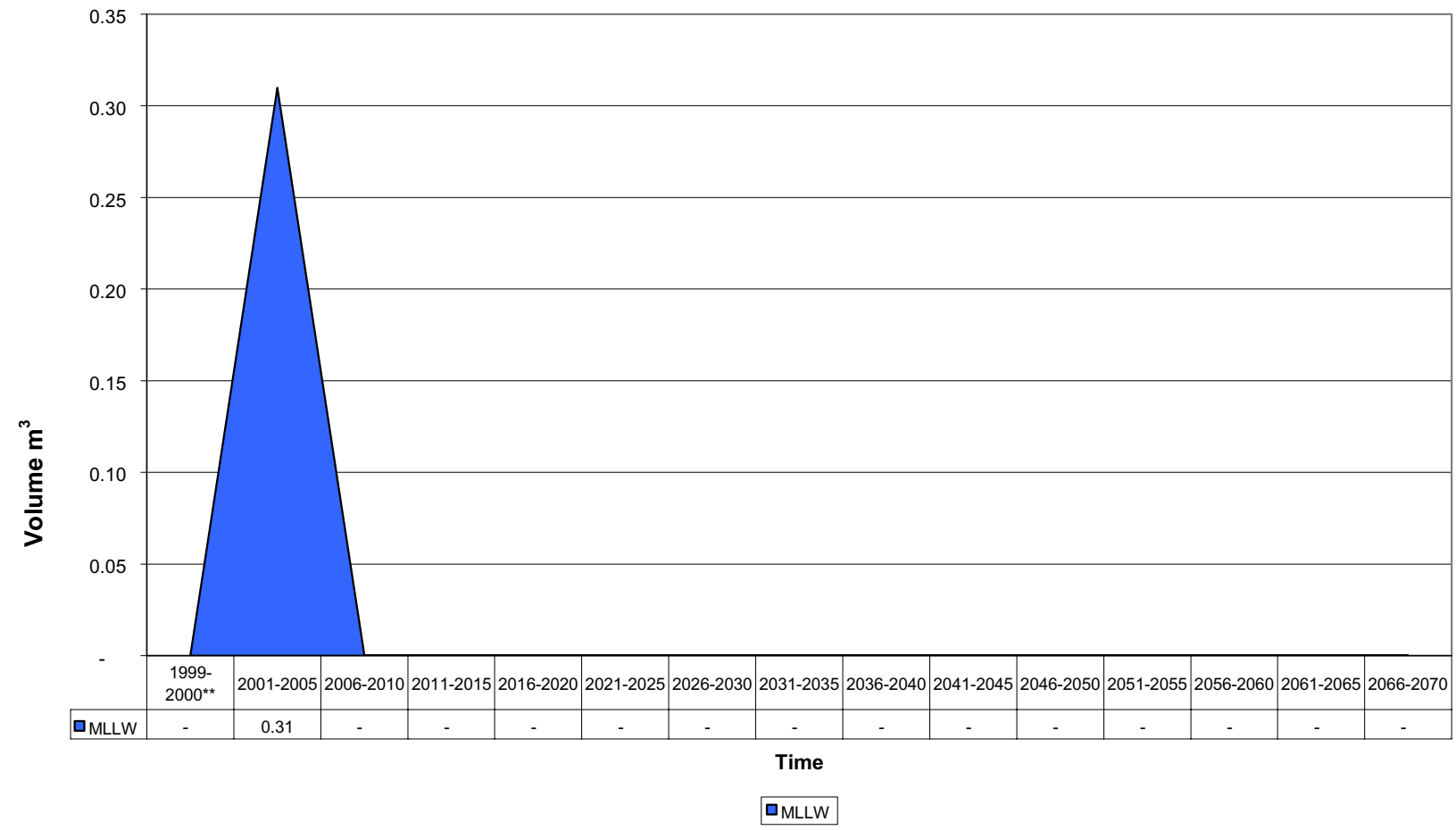

Total life-cycle values per 7/30/99 Stream Disposition Data (SDD) (http://id.inel.gov/avs/).

** Represents data for a two year period. 


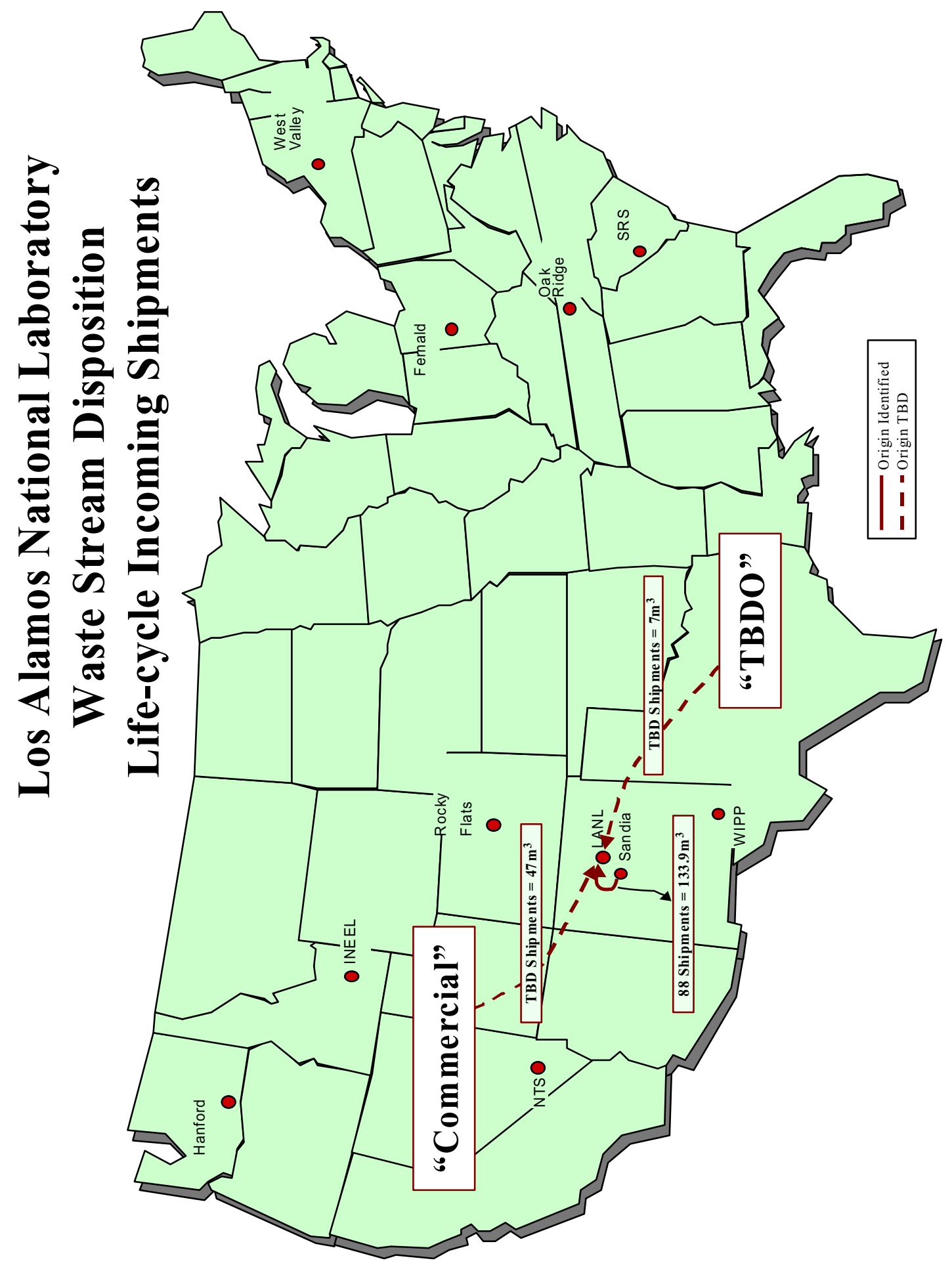



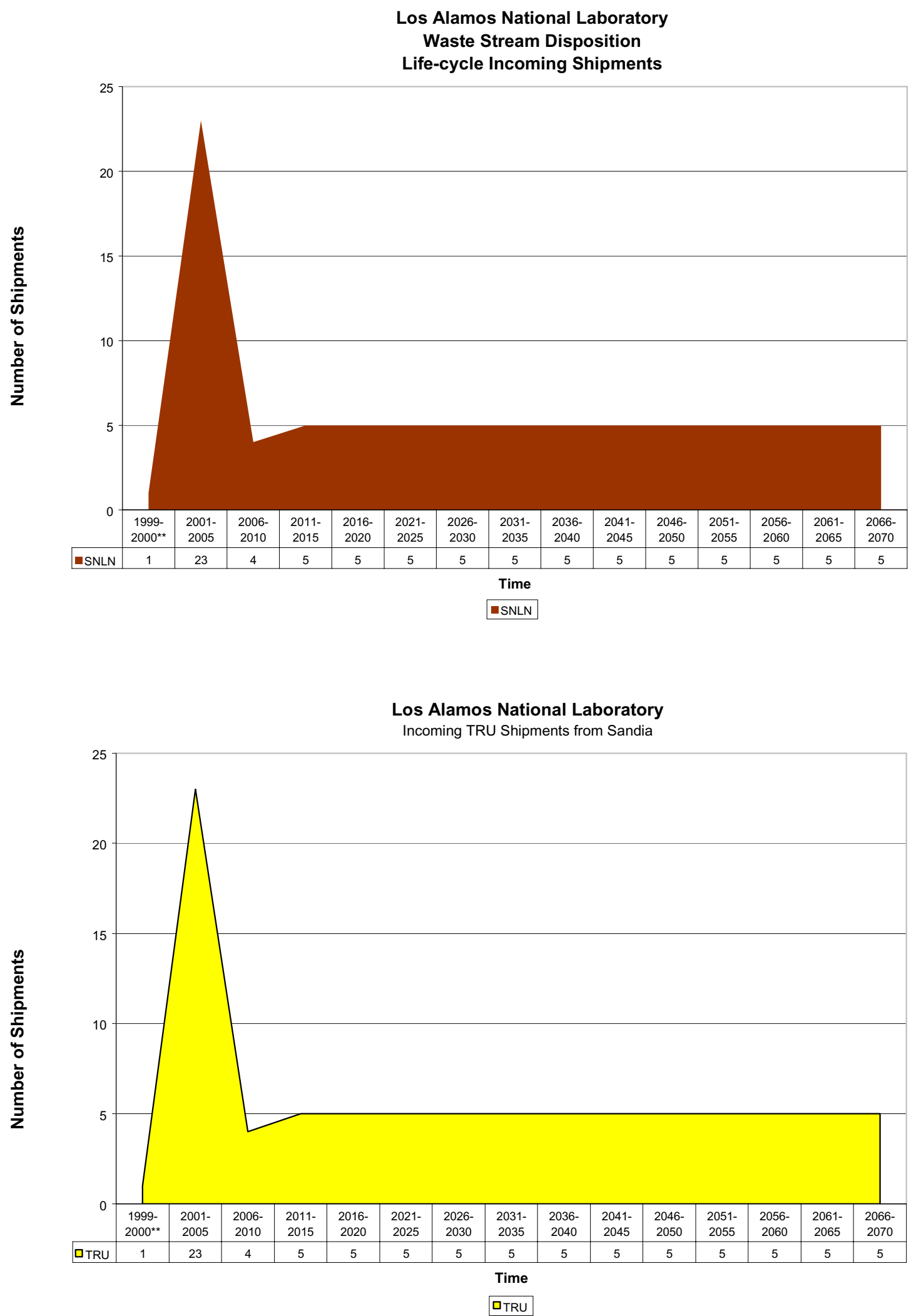

Total life-cycle values per 7/30/99 Stream Disposition Data (SDD) (http://id.inel.gov/avs/).

** Represents data for a two year period. 


\section{Los Alamos National Laboratory \\ Waste Stream Disposition \\ Life-cycle Incoming Volume}

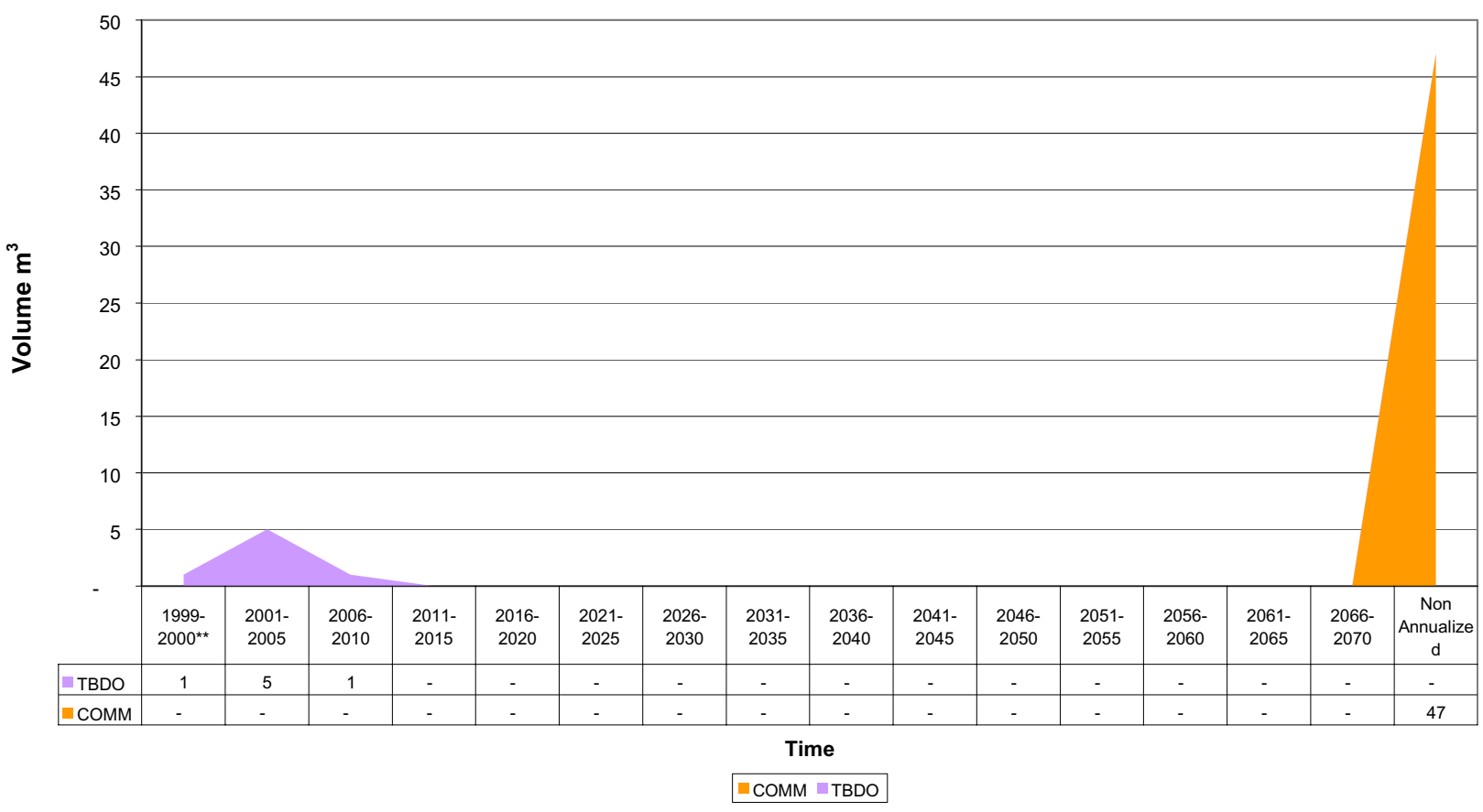

Los Alamos National Laboratory

Incoming LLW Volume from "Commercial"

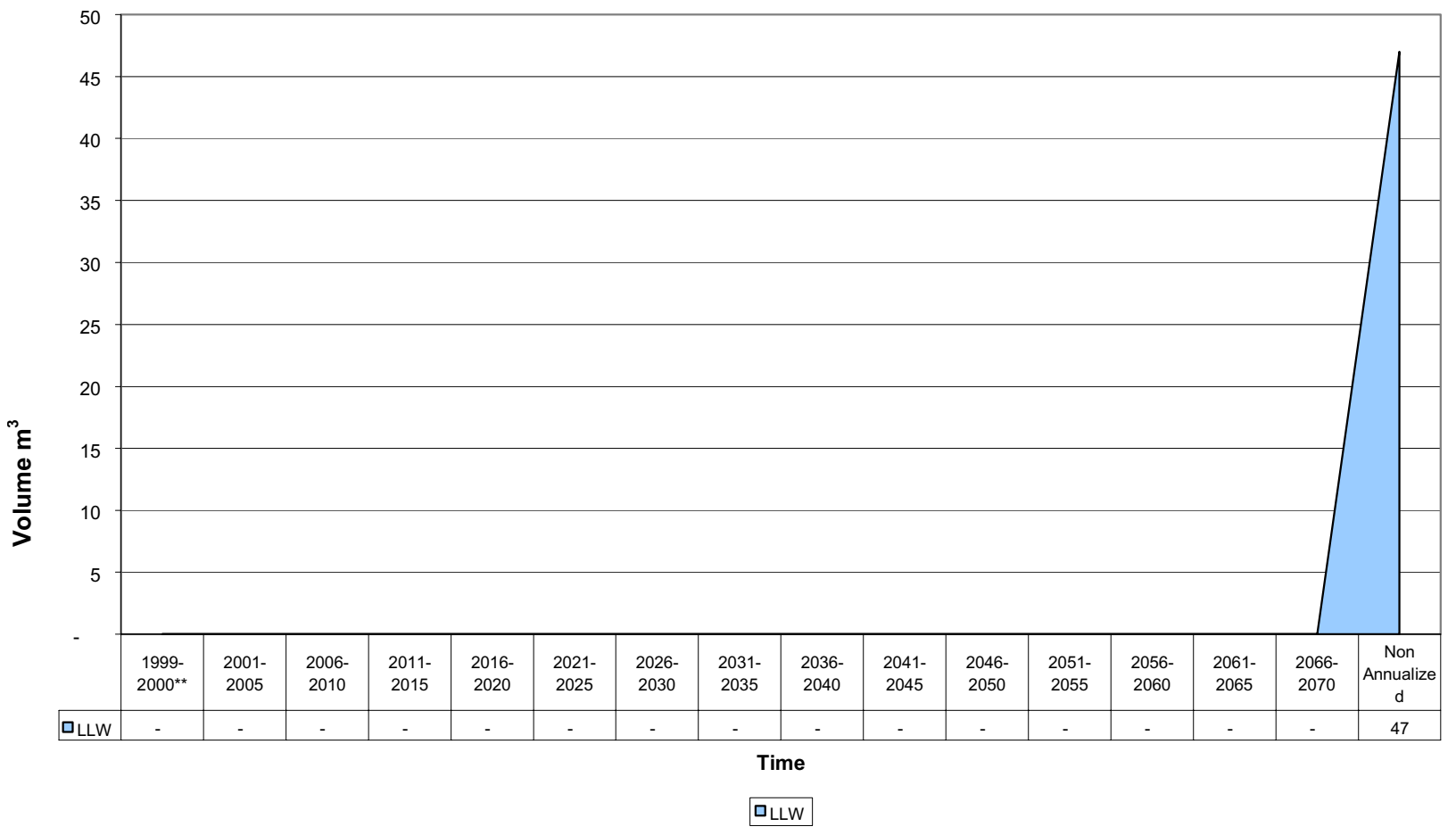

Total life-cycle values per 7/30/99 Stream Disposition Data (SDD) (http://id.inel.gov/avs/).

** Represents data for a two year period. 


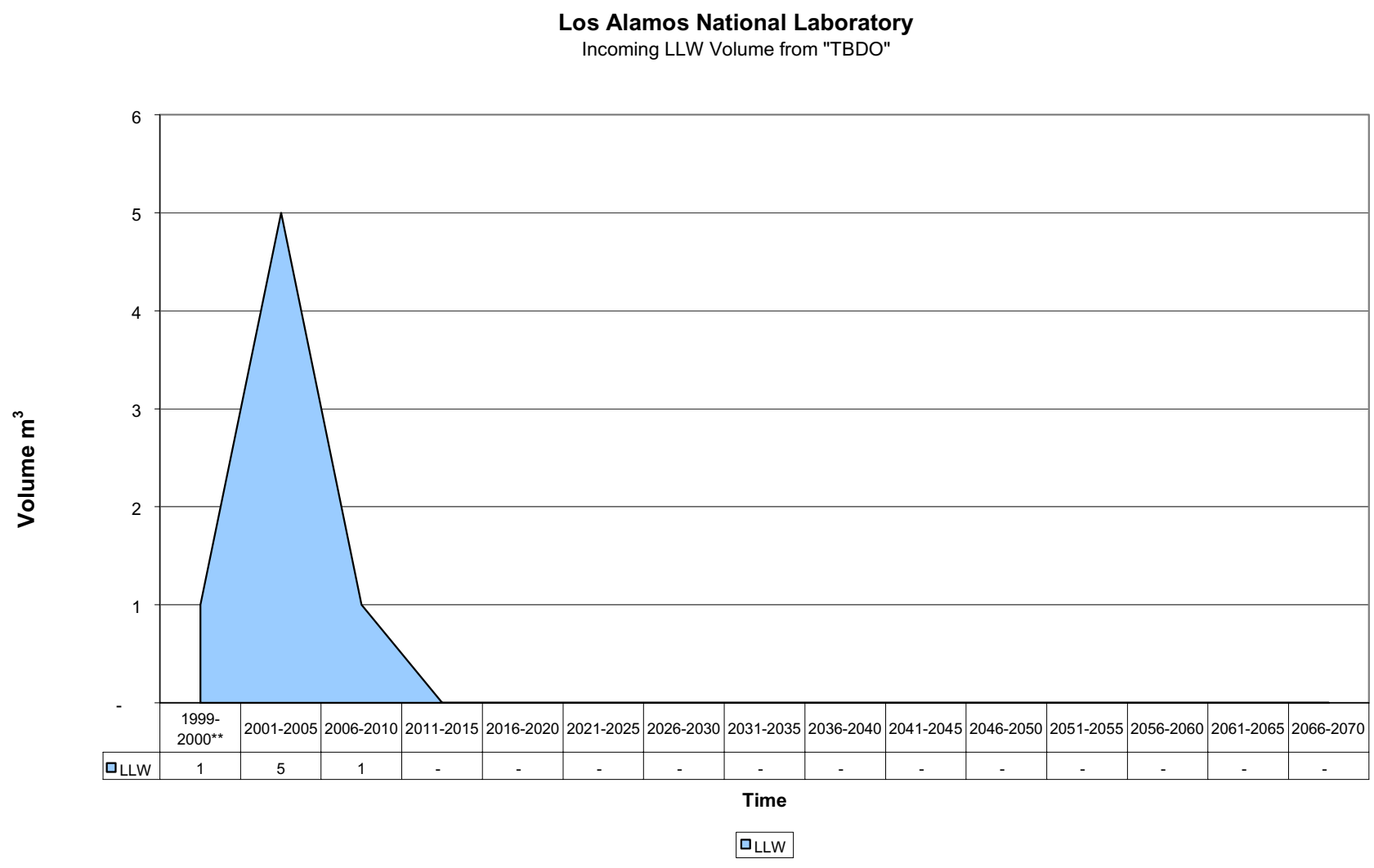

Total life-cycle values per 7/30/99 Stream Disposition Data (SDD) (http://id.inel.gov/avs/).

** Represents data for a two year period. 


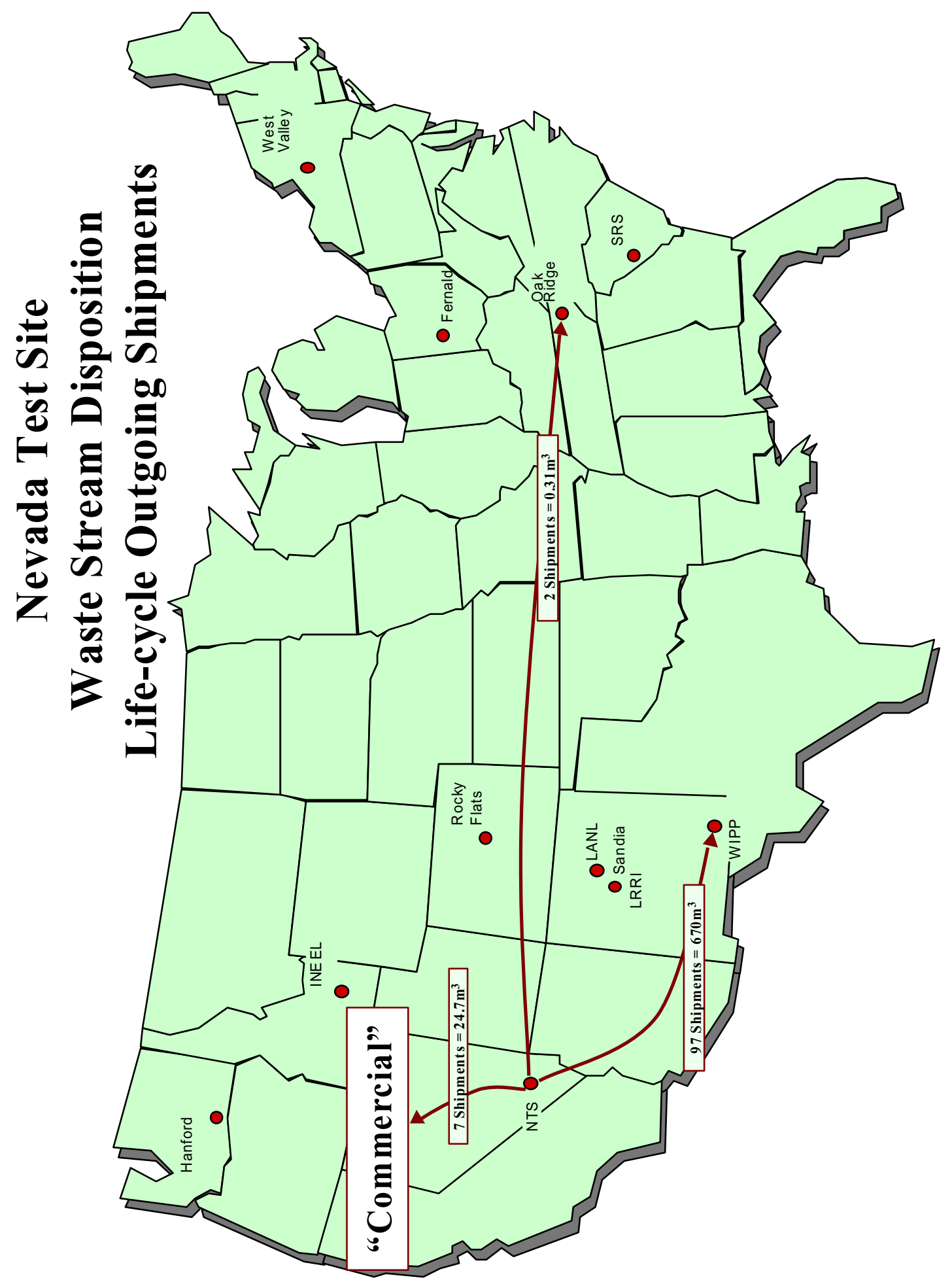


Nevada Test Site

Waste Stream Disposition

Life-cycle Outgoing Shipments

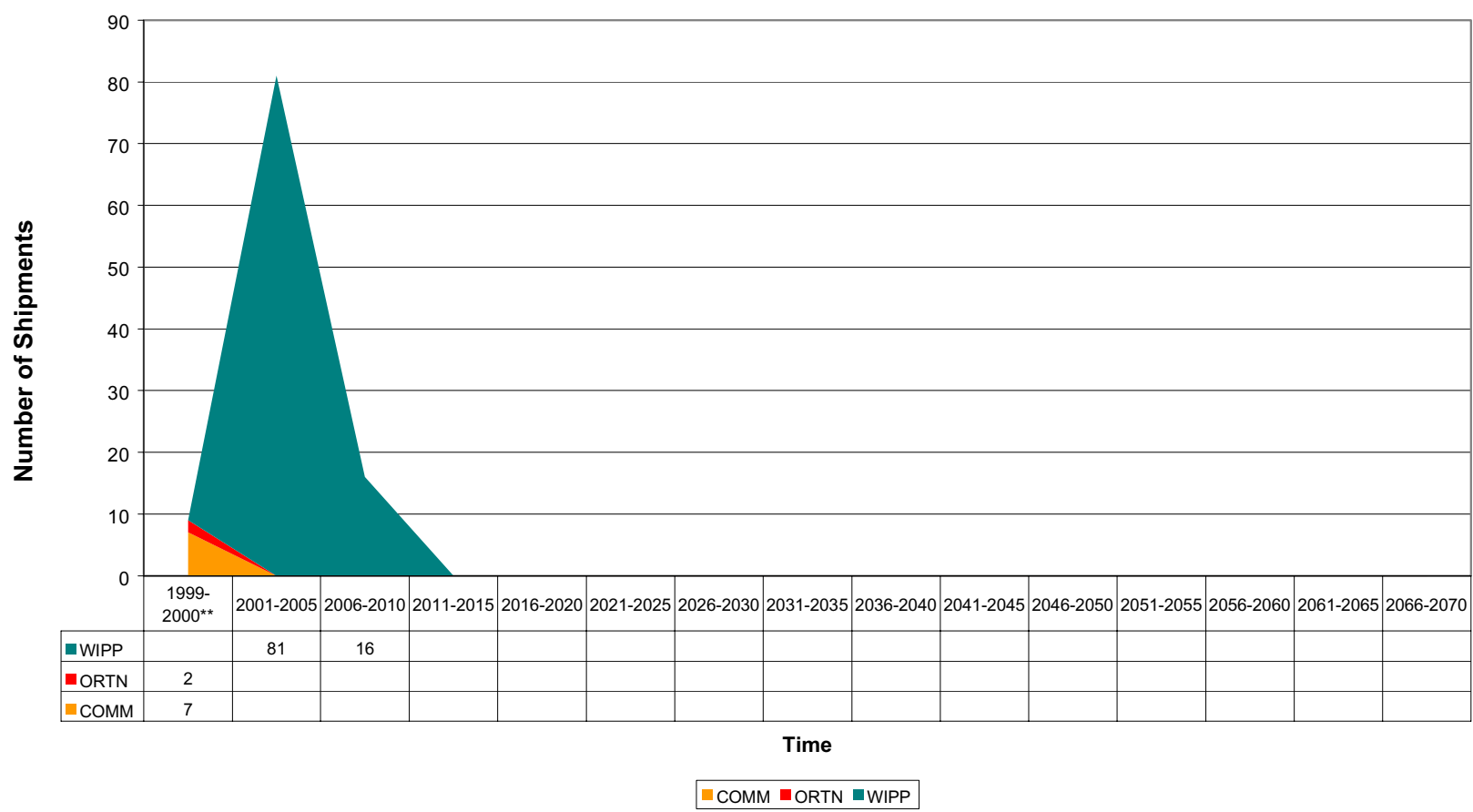

Nevada Test Site

Outgoing MLLW Shipments to "Commercial"

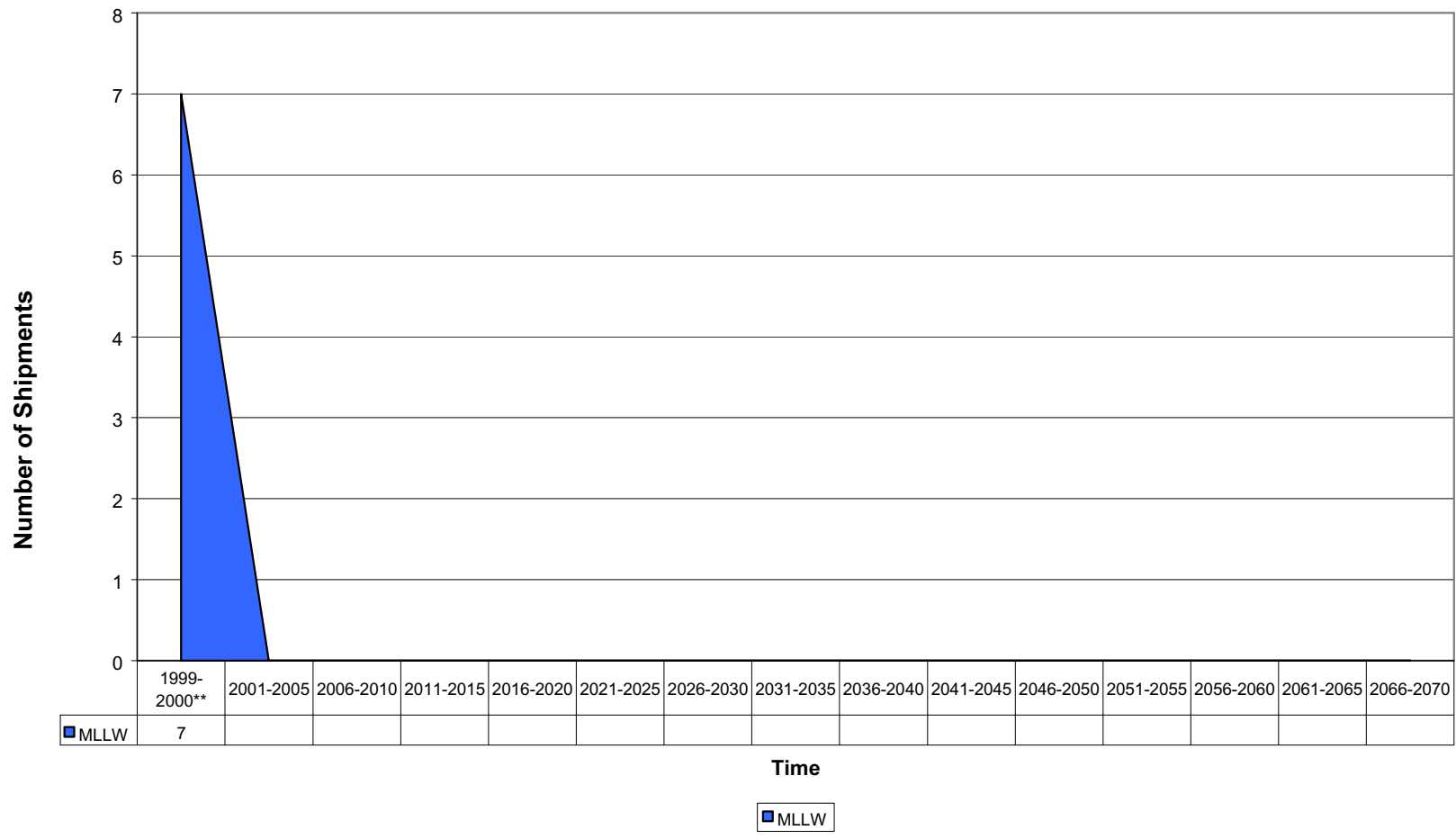

Total life-cycle values per 7/30/99 Stream Disposition Data (SDD) (http://id.inel.gov/avs/).

** Represents data for a two year period. 
Nevada Test Site

Outgoing MLLW Shipments to Oak Ridge

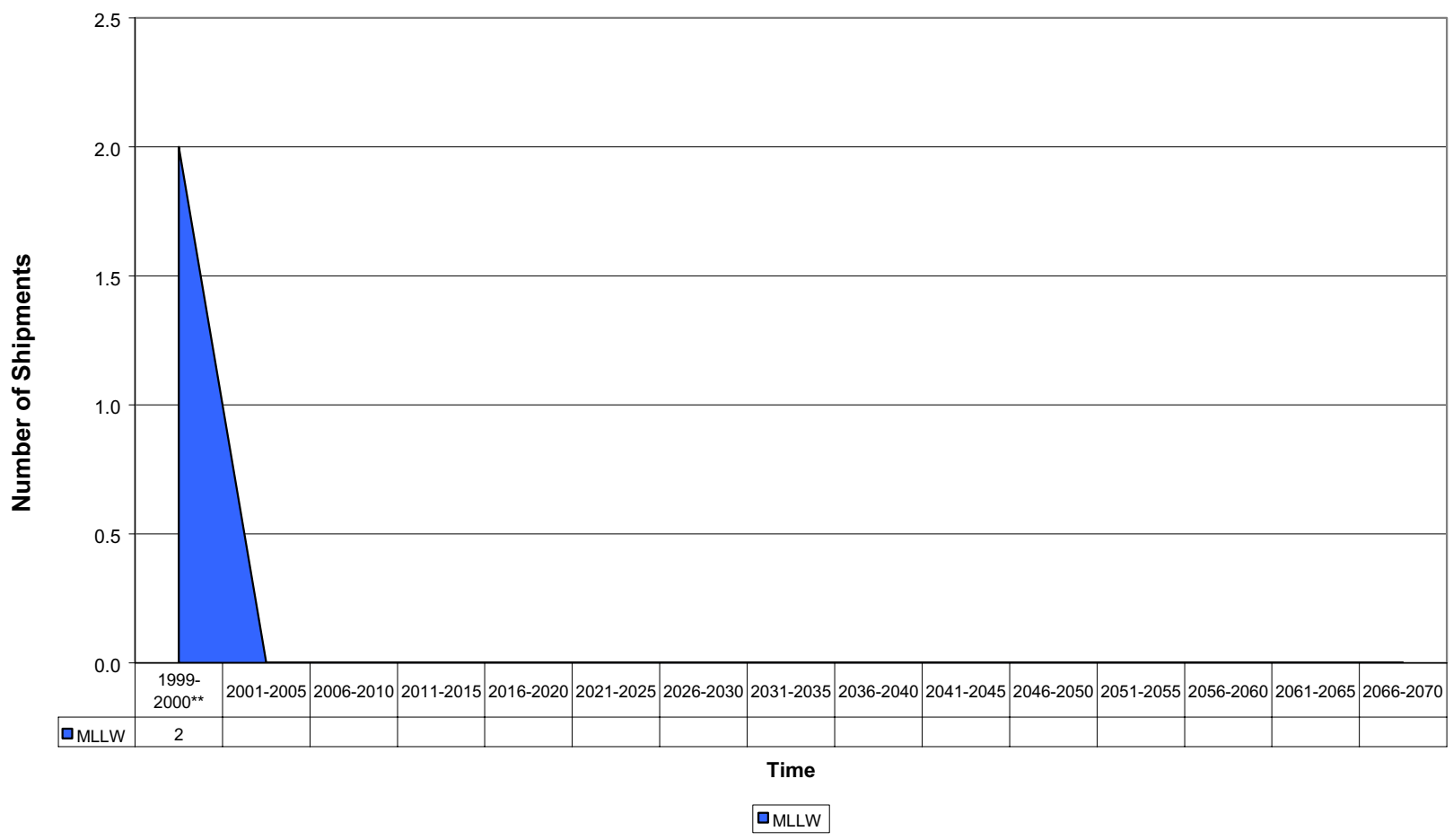

Nevada Test Site

Outgoing TRU Shipments to WIPP

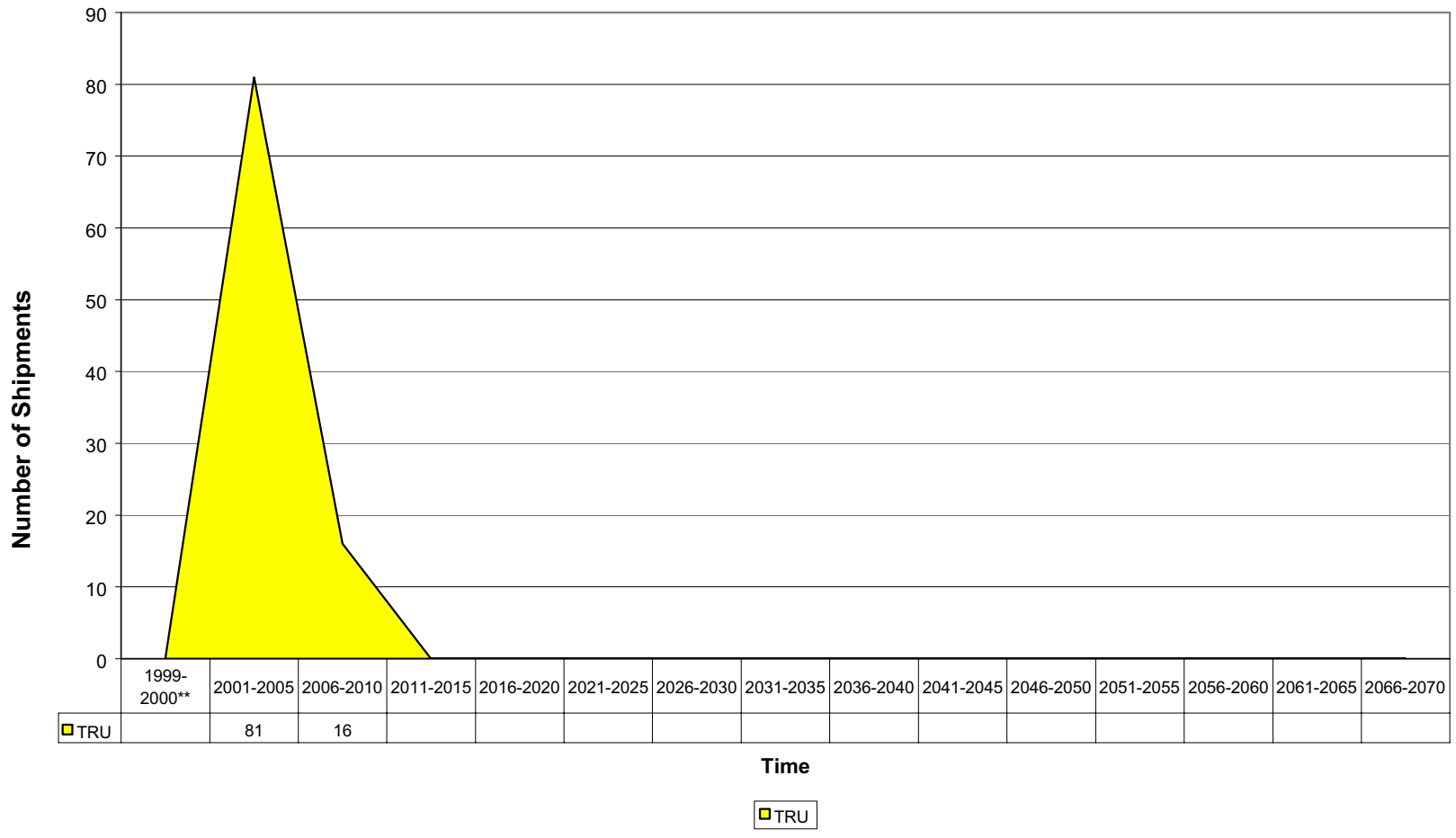

Total life-cycle values per 7/30/99 Stream Disposition Data (SDD) (http://id.inel.gov/avs/).

** Represents data for a two year period. 



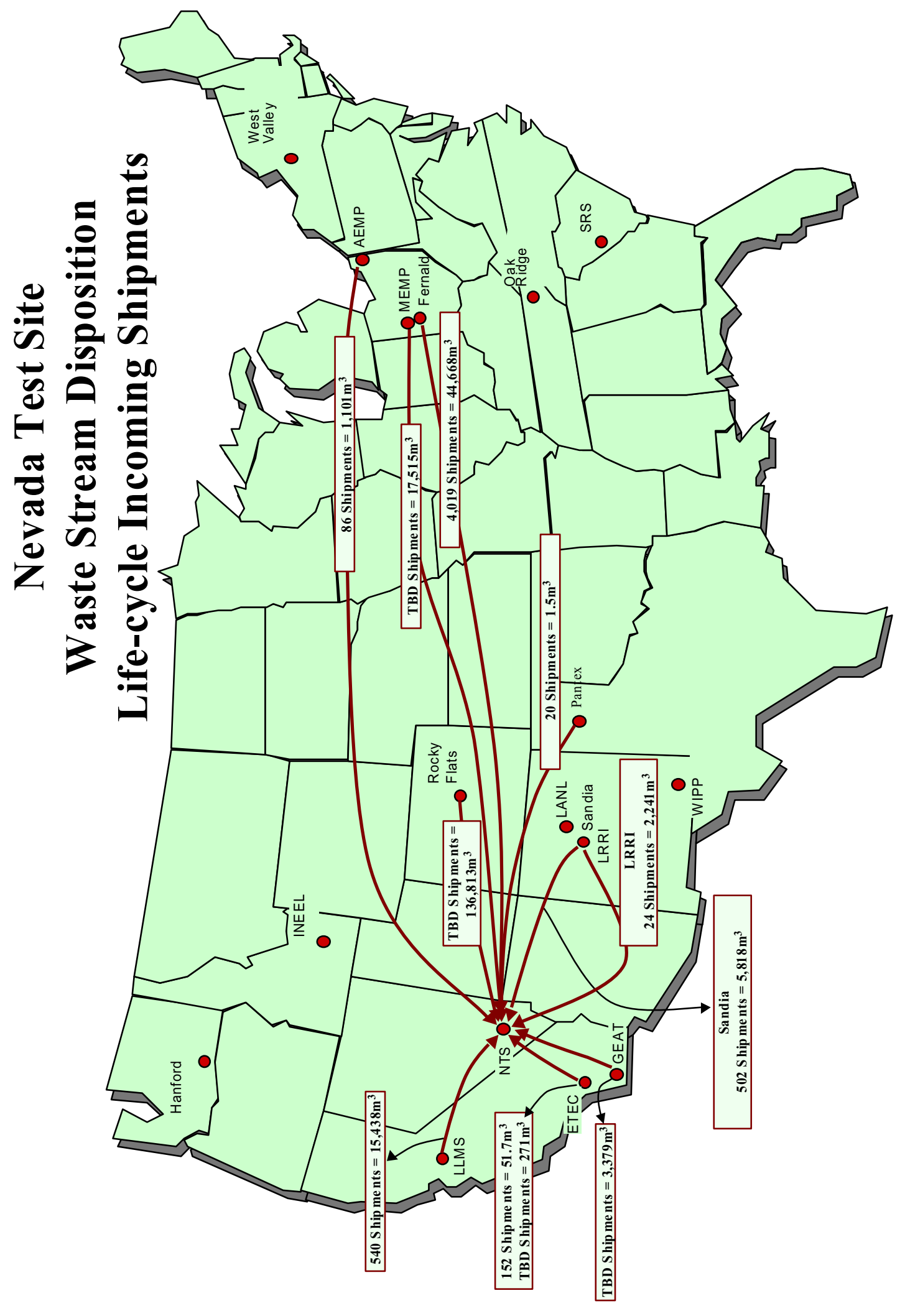



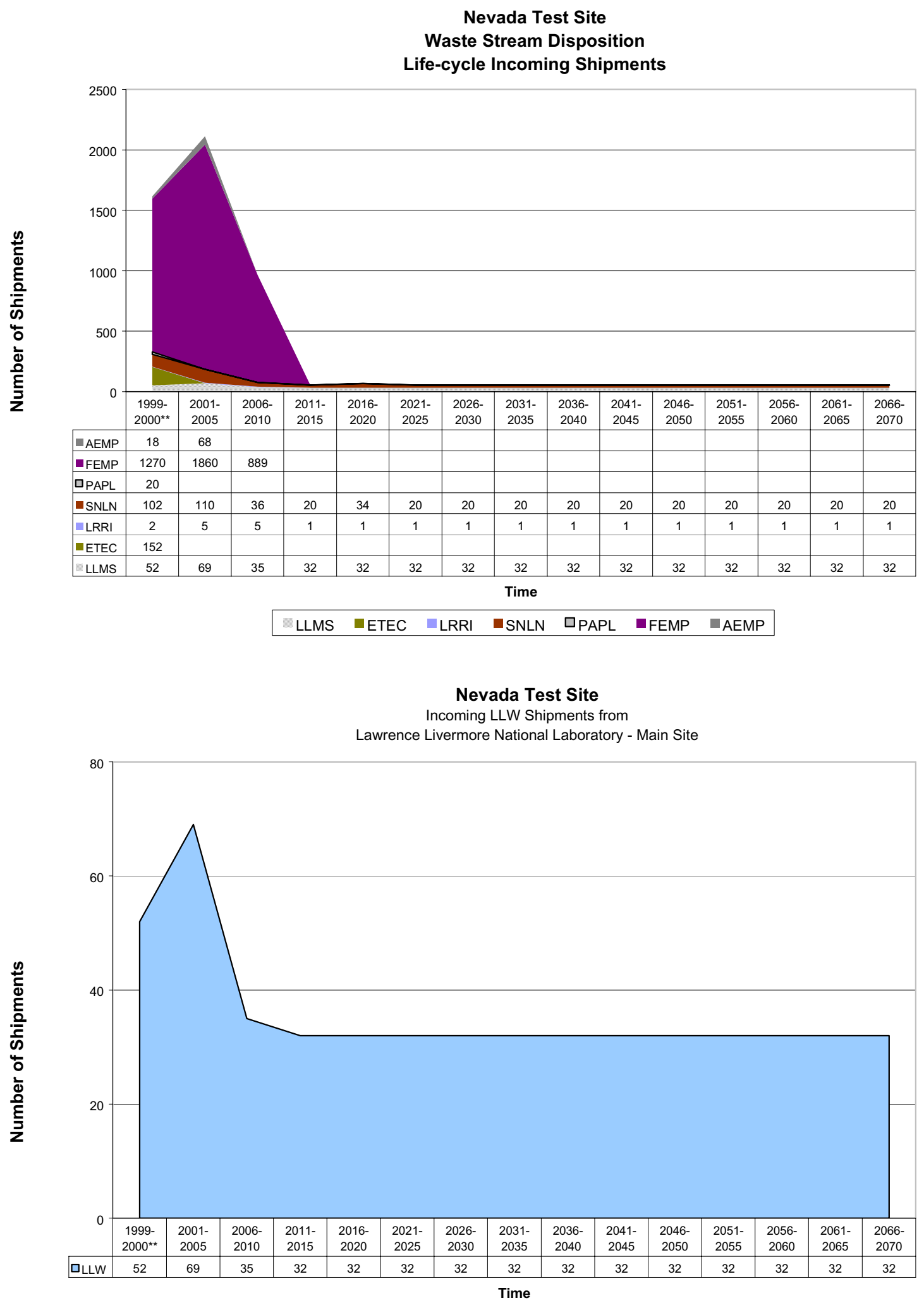

QLLW

Total life-cycle values per 7/30/99 Stream Disposition Data (SDD) (http://id.inel.gov/avs/).

** Represents data for a two year period. 

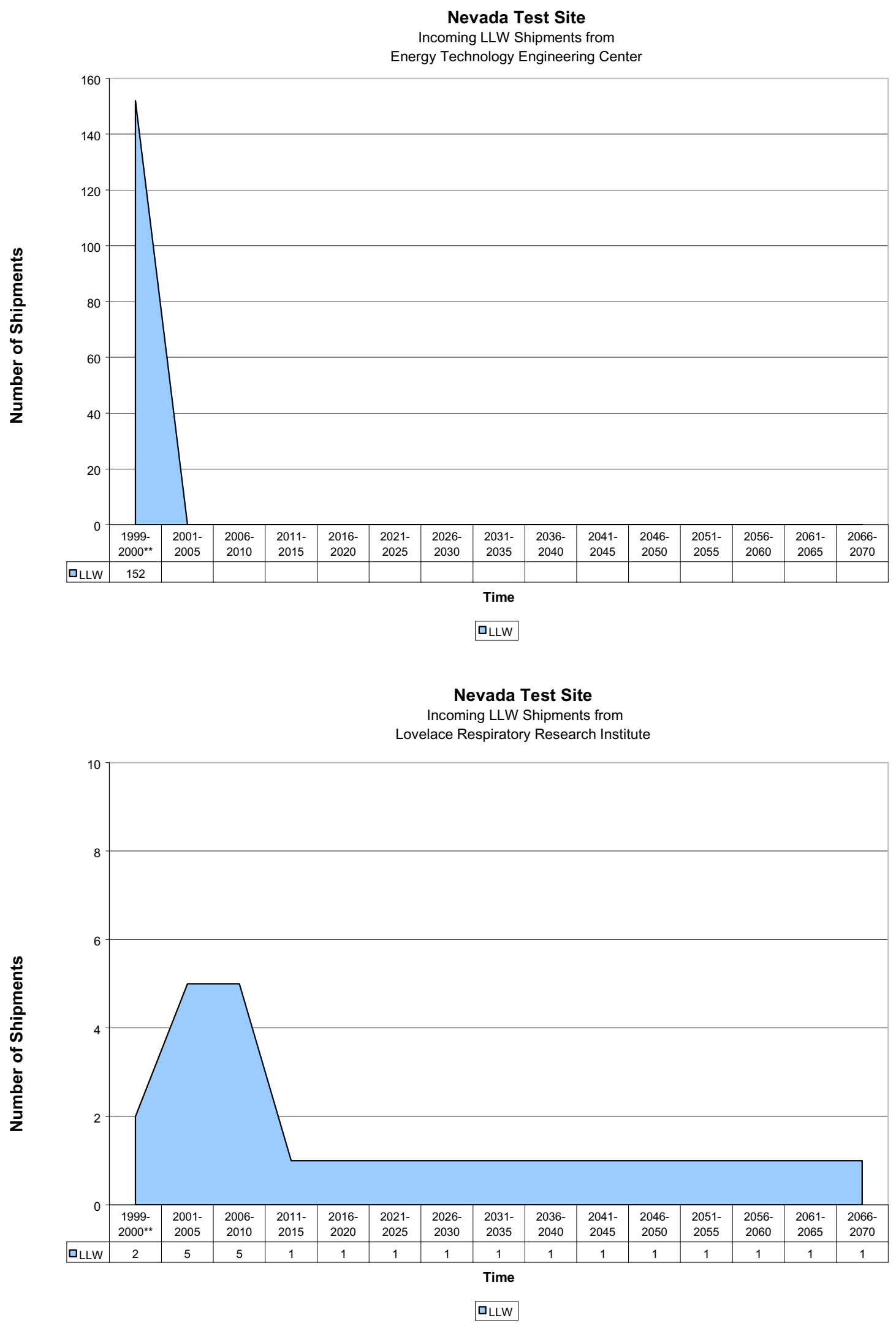

Total life-cycle values per 7/30/99 Stream Disposition Data (SDD) (http://id.inel.gov/avs/).

** Represents data for a two year period. 


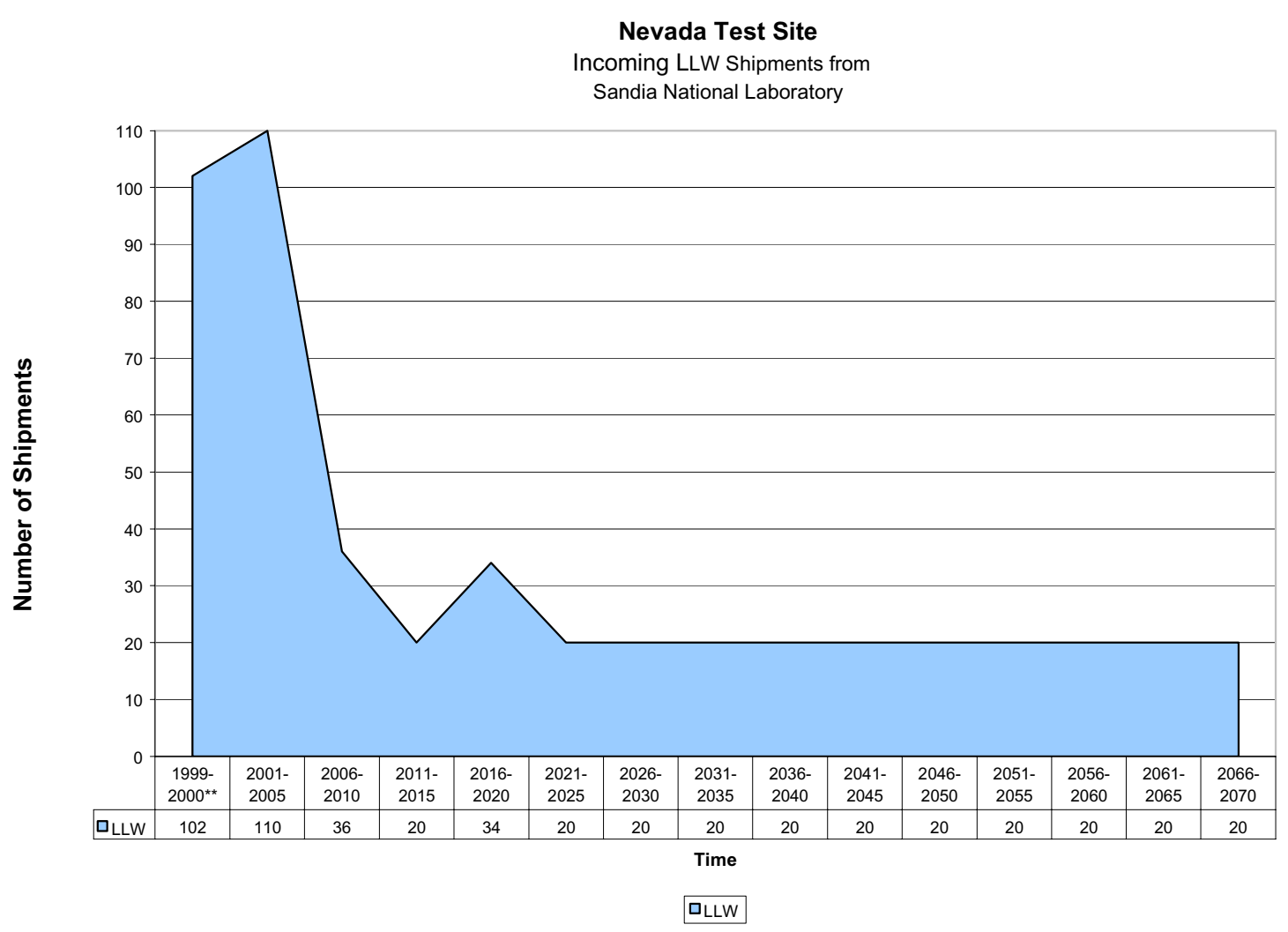

Nevada Test Site

Incoming LLW Shipments from Pantex Plant

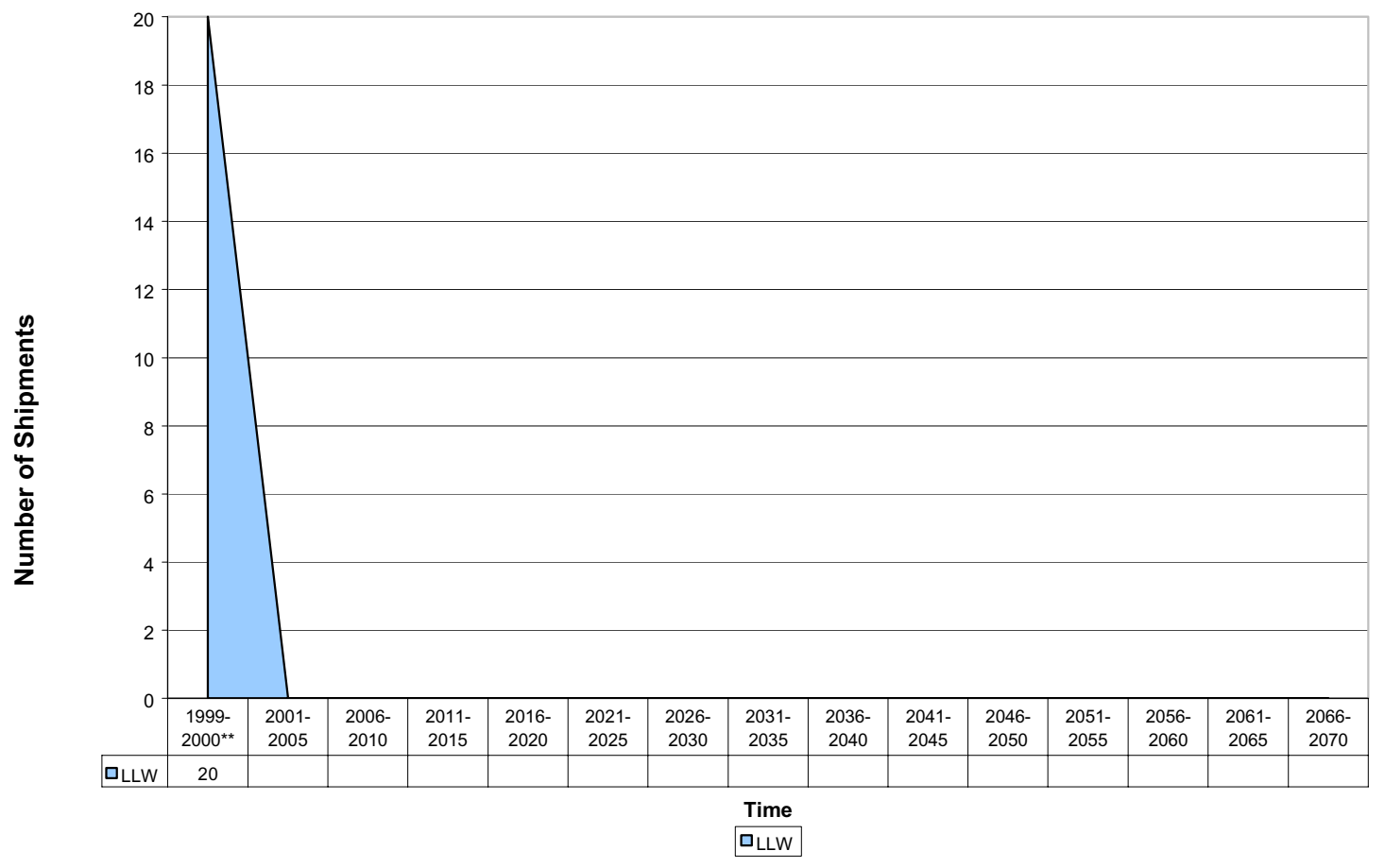

Total life-cycle values per 7/30/99 Stream Disposition Data (SDD) (http://id.inel.gov/avs/).

** Represents data for a two year period. 

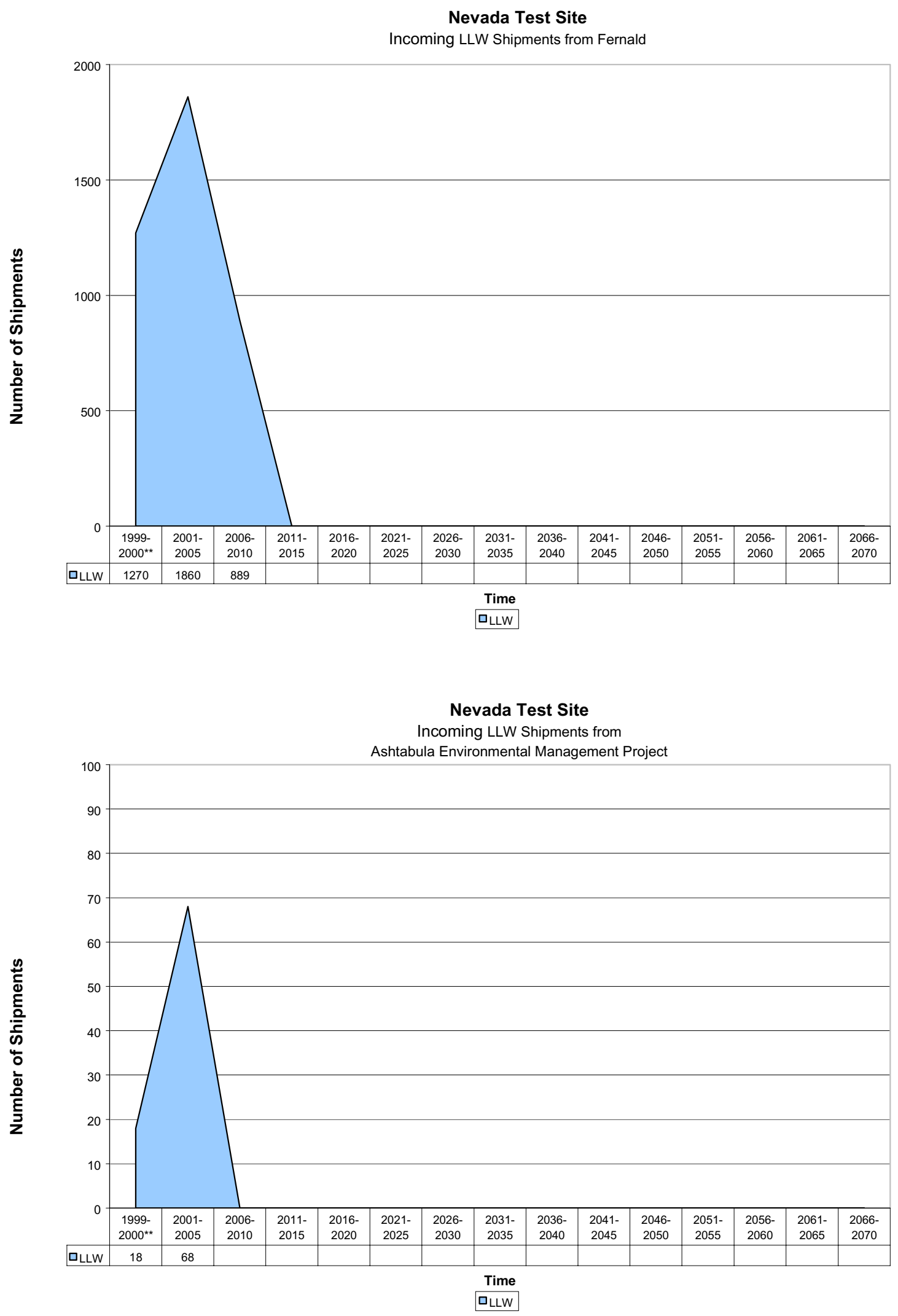

Total life-cycle values per 7/30/99 Stream Disposition Data (SDD) (http://id.inel.gov/avs/).

** Represents data for a two year period. 
Nevada Test Site

Waste Stream Disposition

Life-cycle Incoming Volume

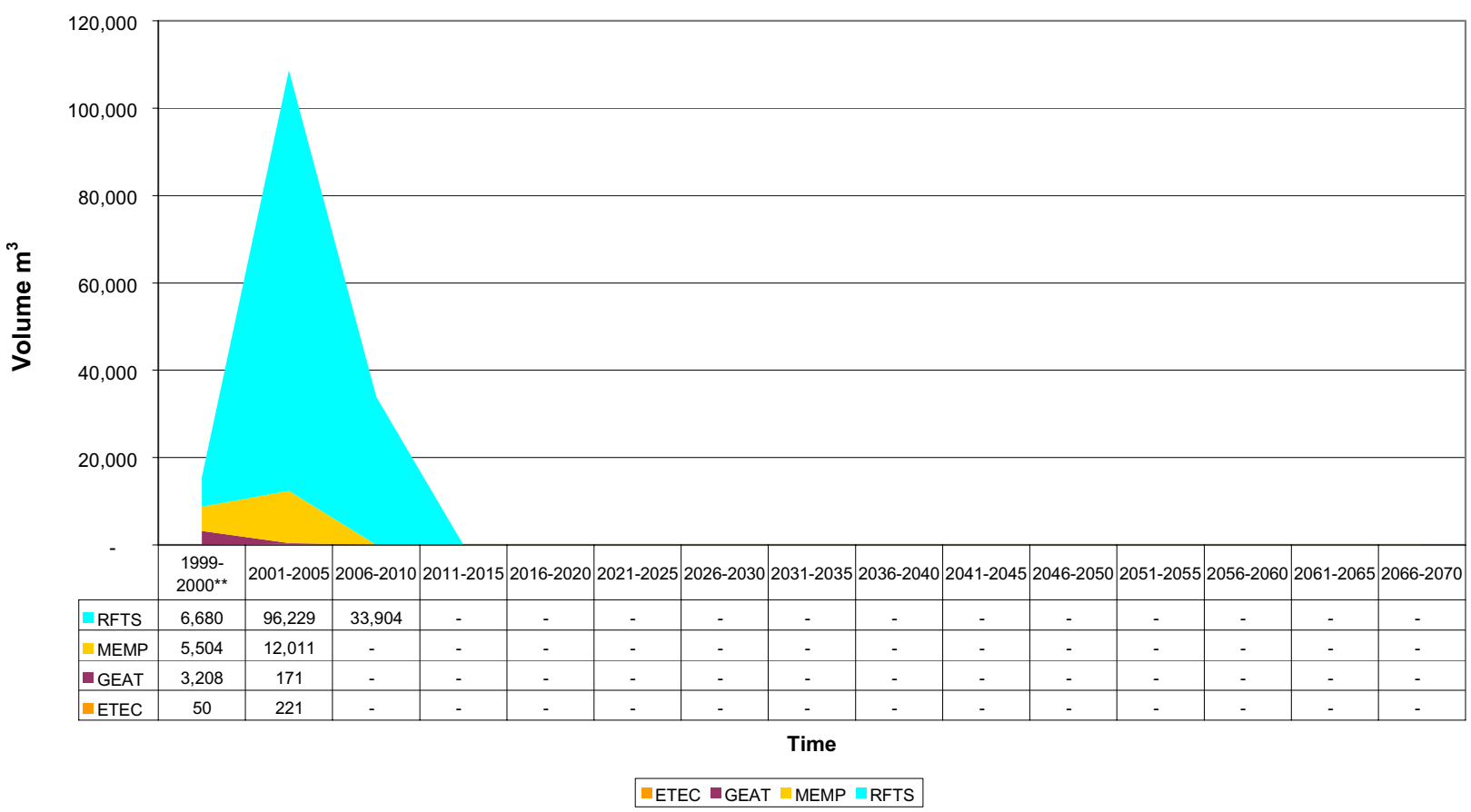

Nevada Test Site

Incoming LLW Volume from Energy Technology Engineering Center

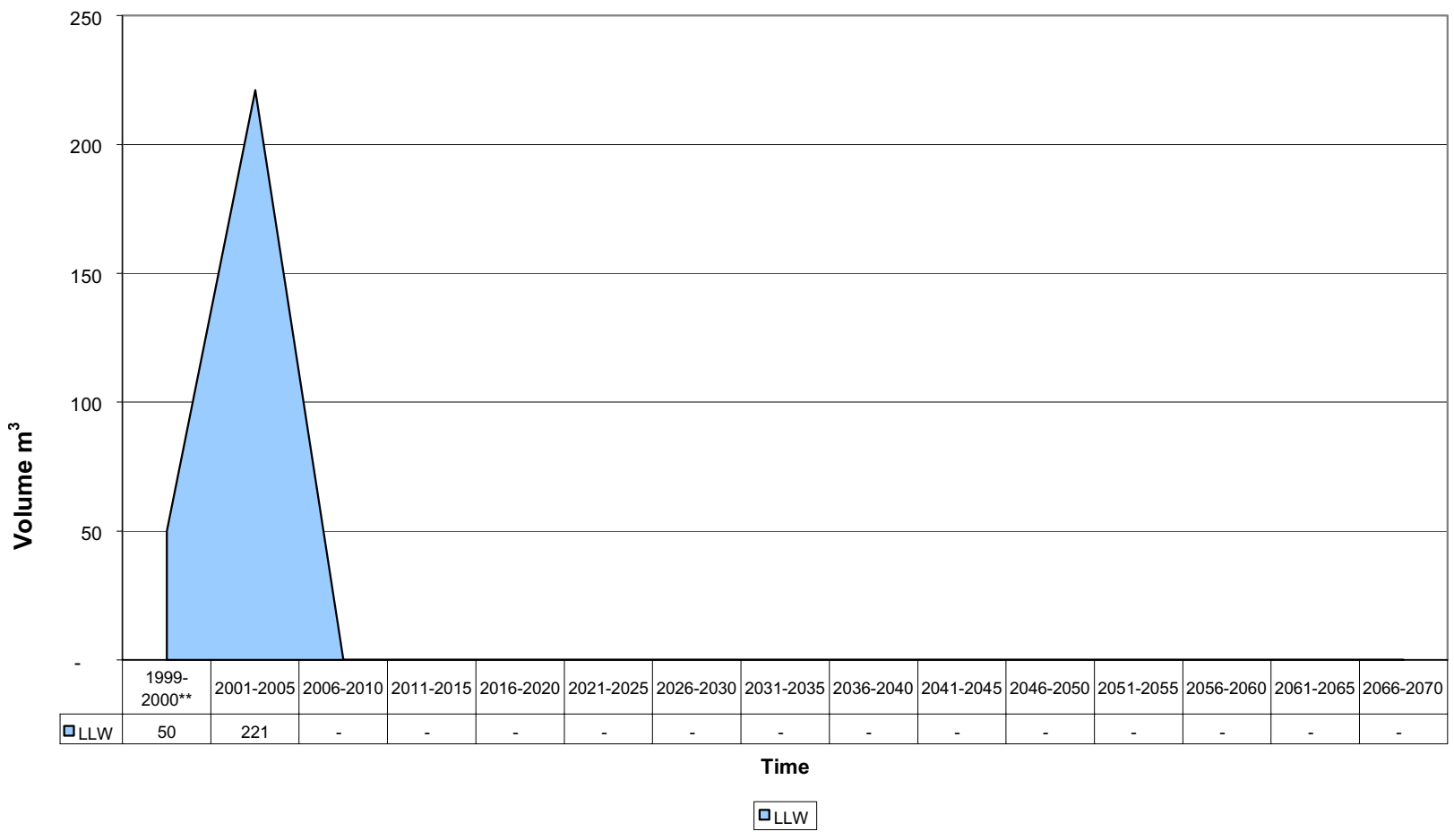

Total life-cycle values per 7/30/99 Stream Disposition Data (SDD) (http://id.inel.gov/avs/).

** Represents data for a two year period. 
Nevada Test Site

Incoming LLW Volume from General Atomics

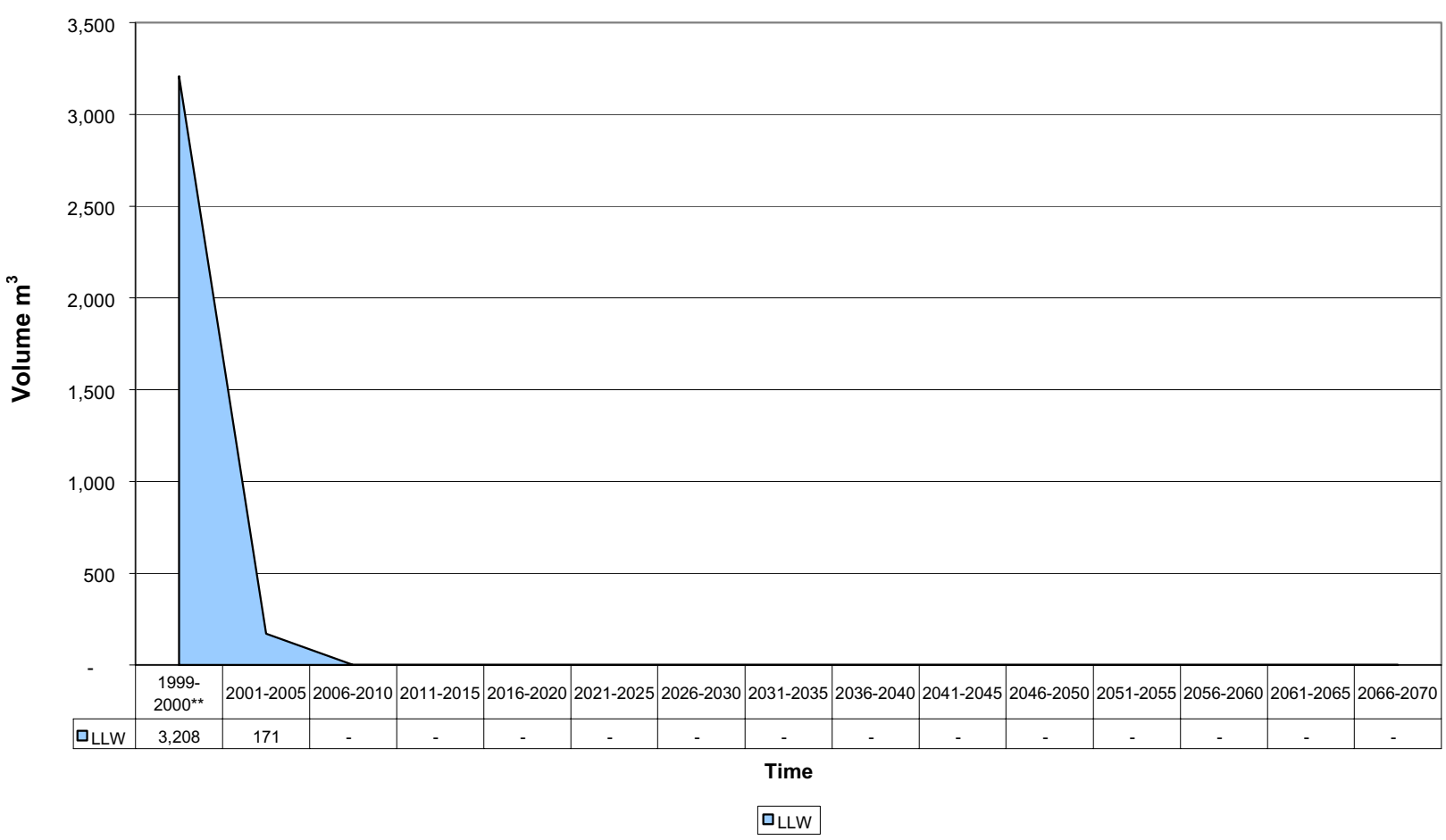

Nevada Test Site

Incoming LLW Volume from Miamisburg Environmental Management Project

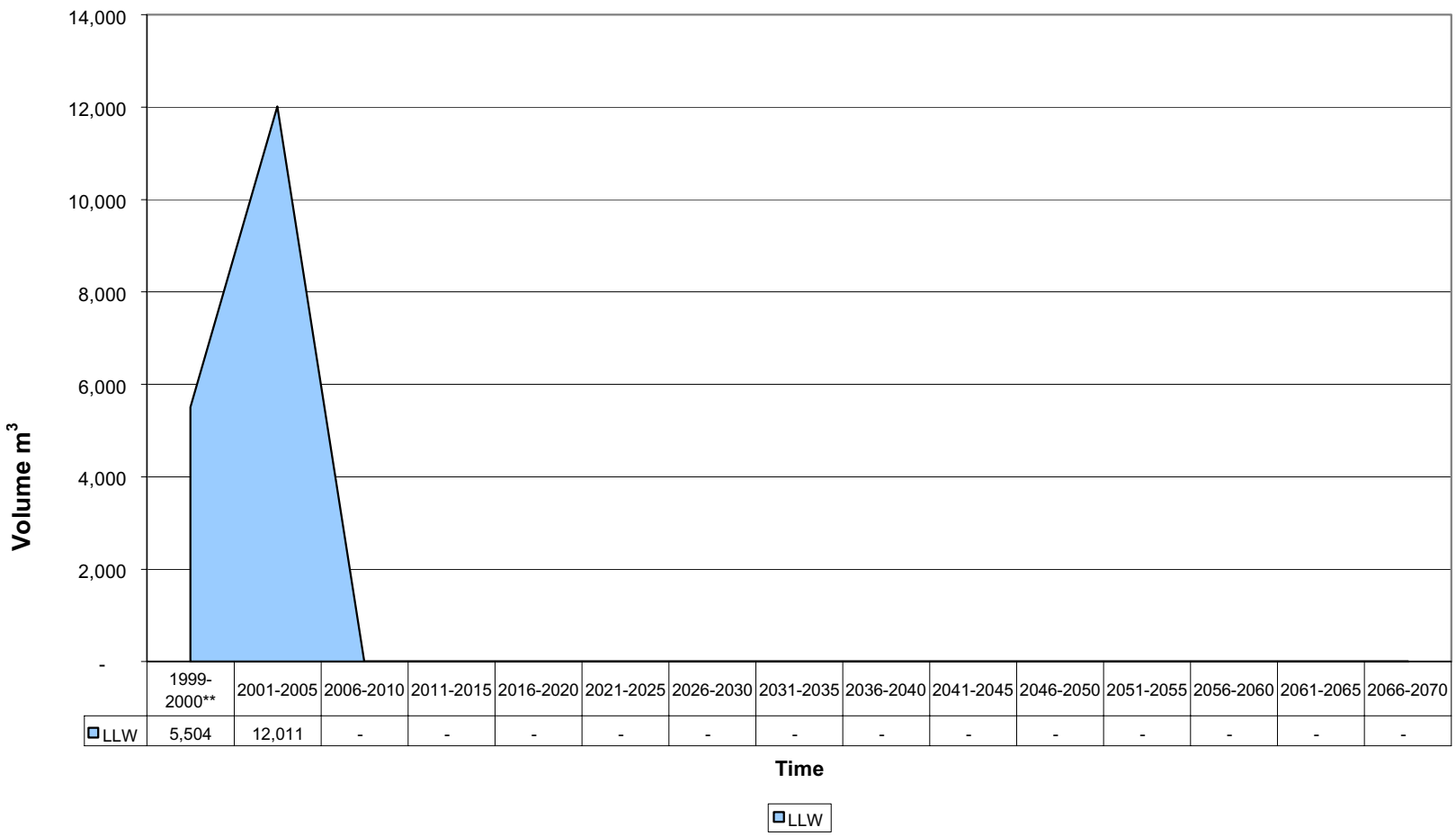

Total life-cycle values per 7/30/99 Stream Disposition Data (SDD) (http://id.inel.gov/avs/).

** Represents data for a two year period. 
Nevada Test Site

Incoming LLW Volume from Rocky Flats

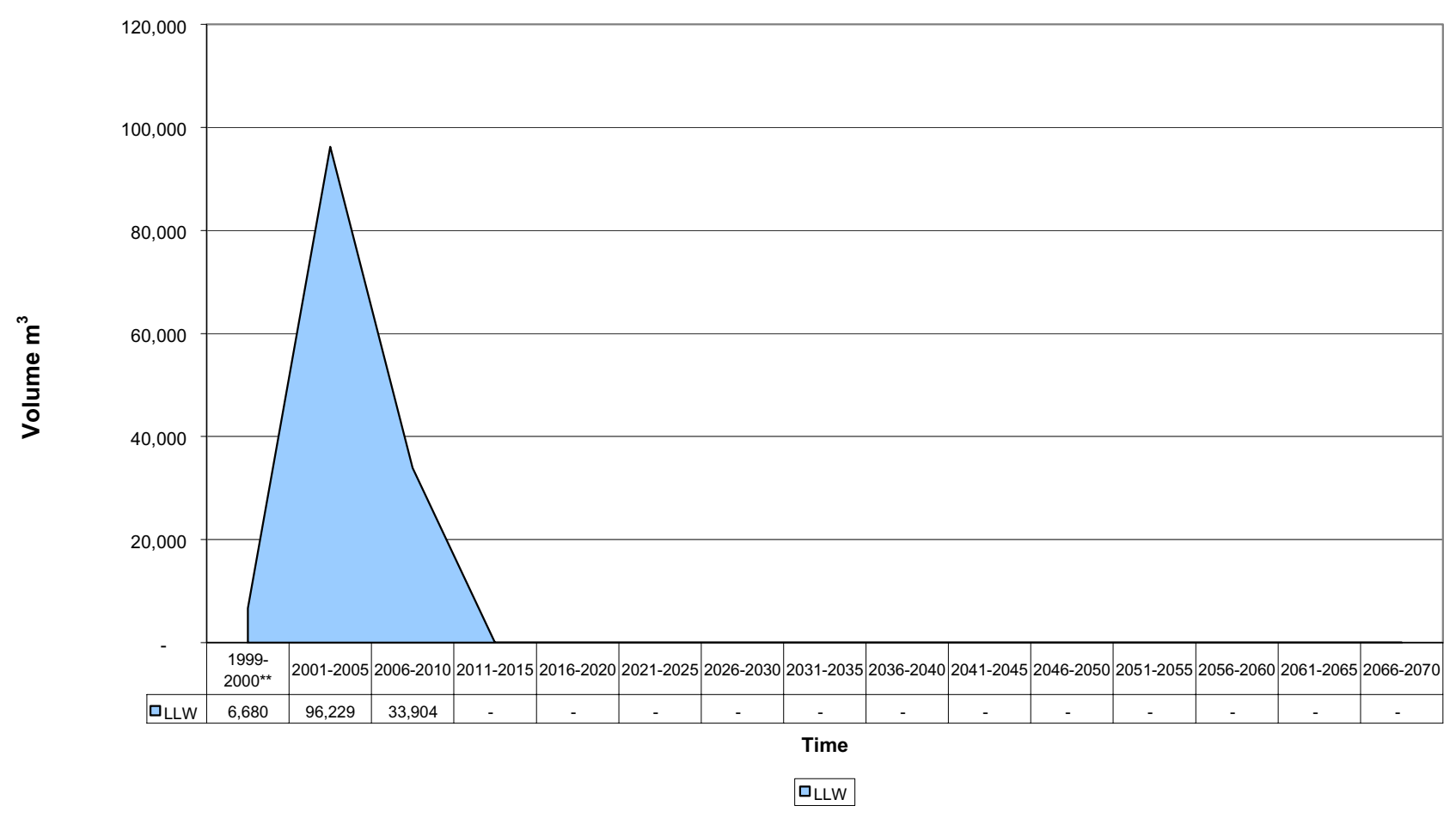

Total life-cycle values per 7/30/99 Stream Disposition Data (SDD) (http://id.inel.gov/avs/).

** Represents data for a two year period. 


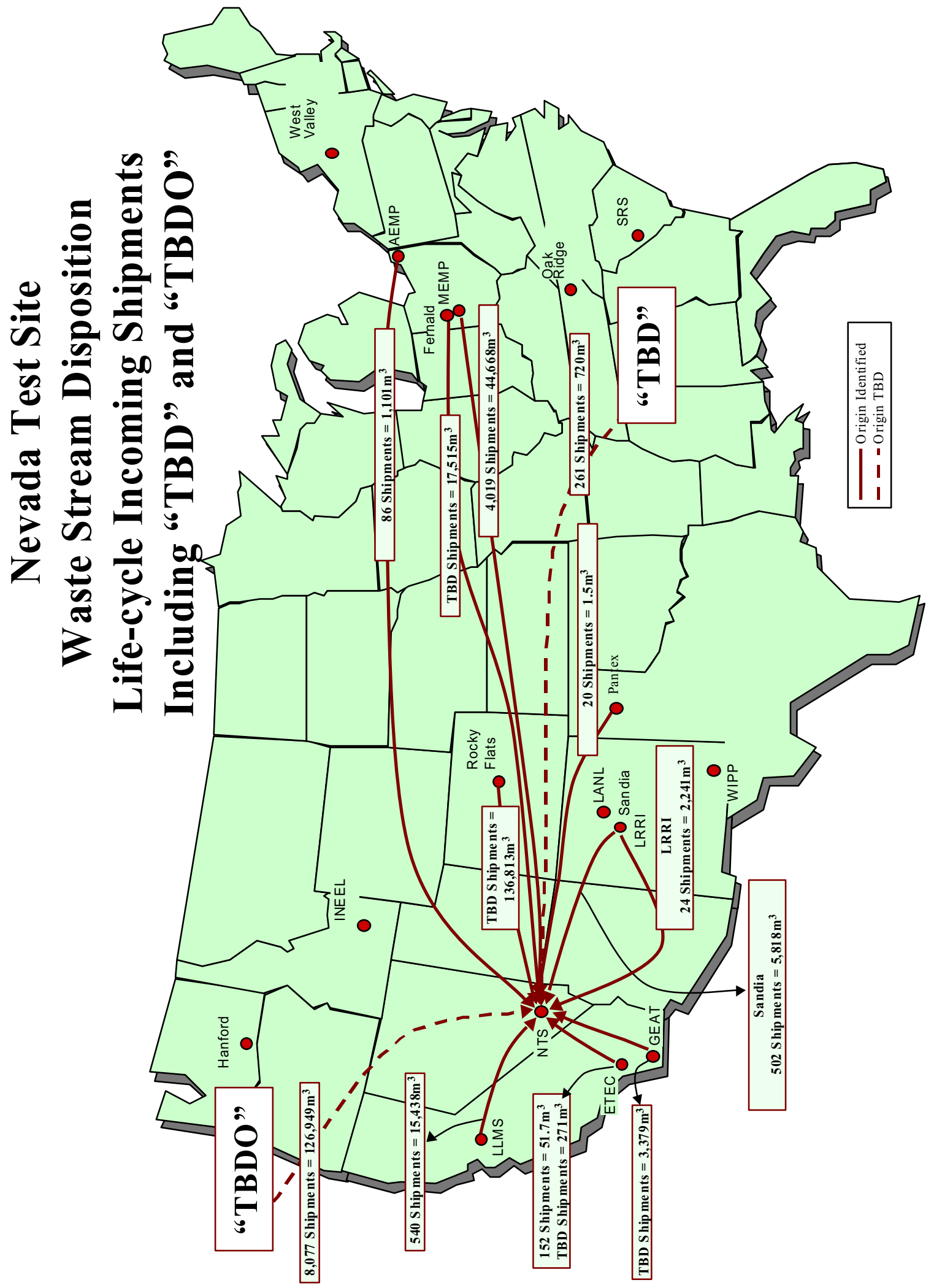




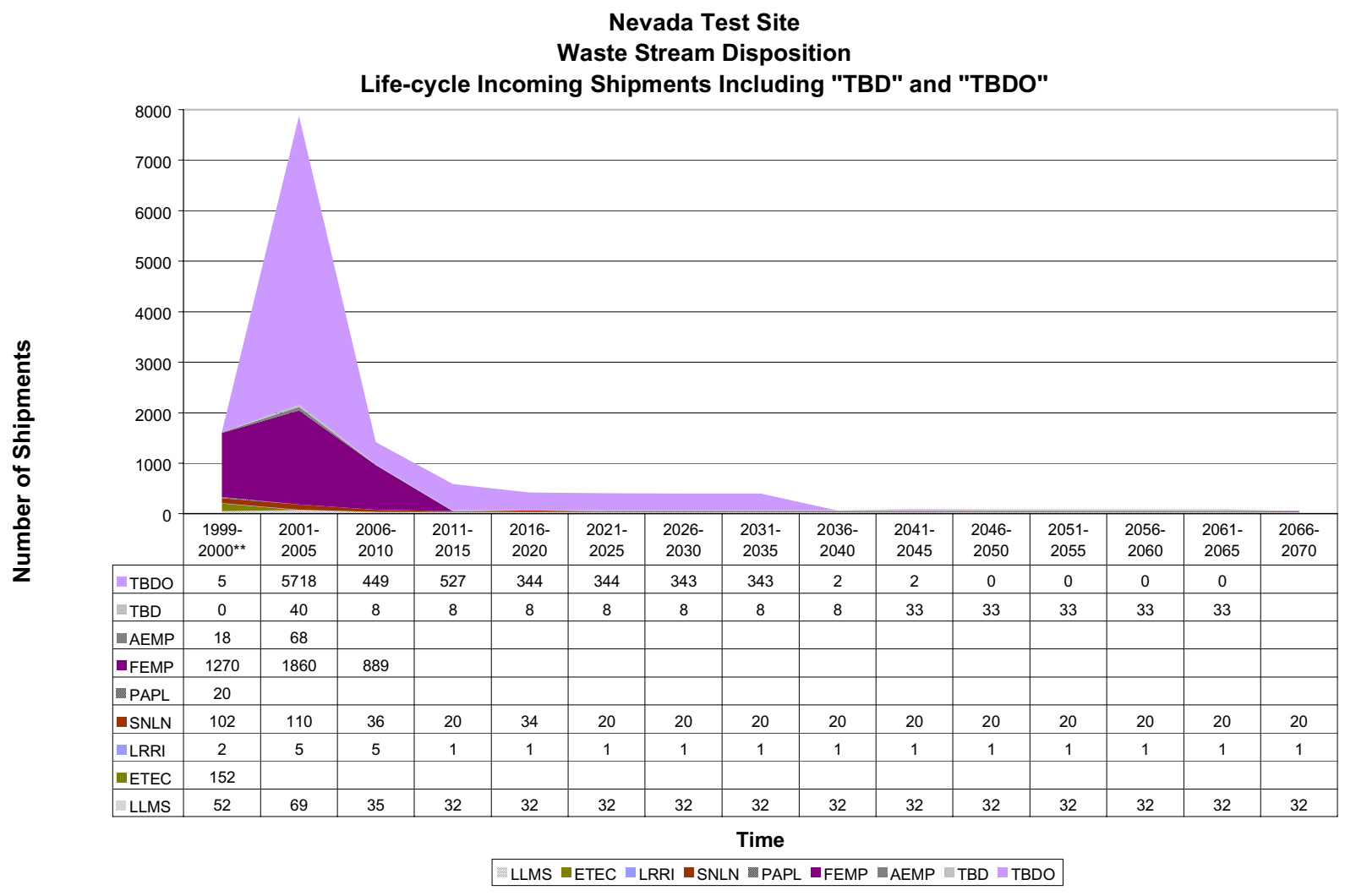

Nevada Test Site

Incoming LLW Shipments from "TBD"

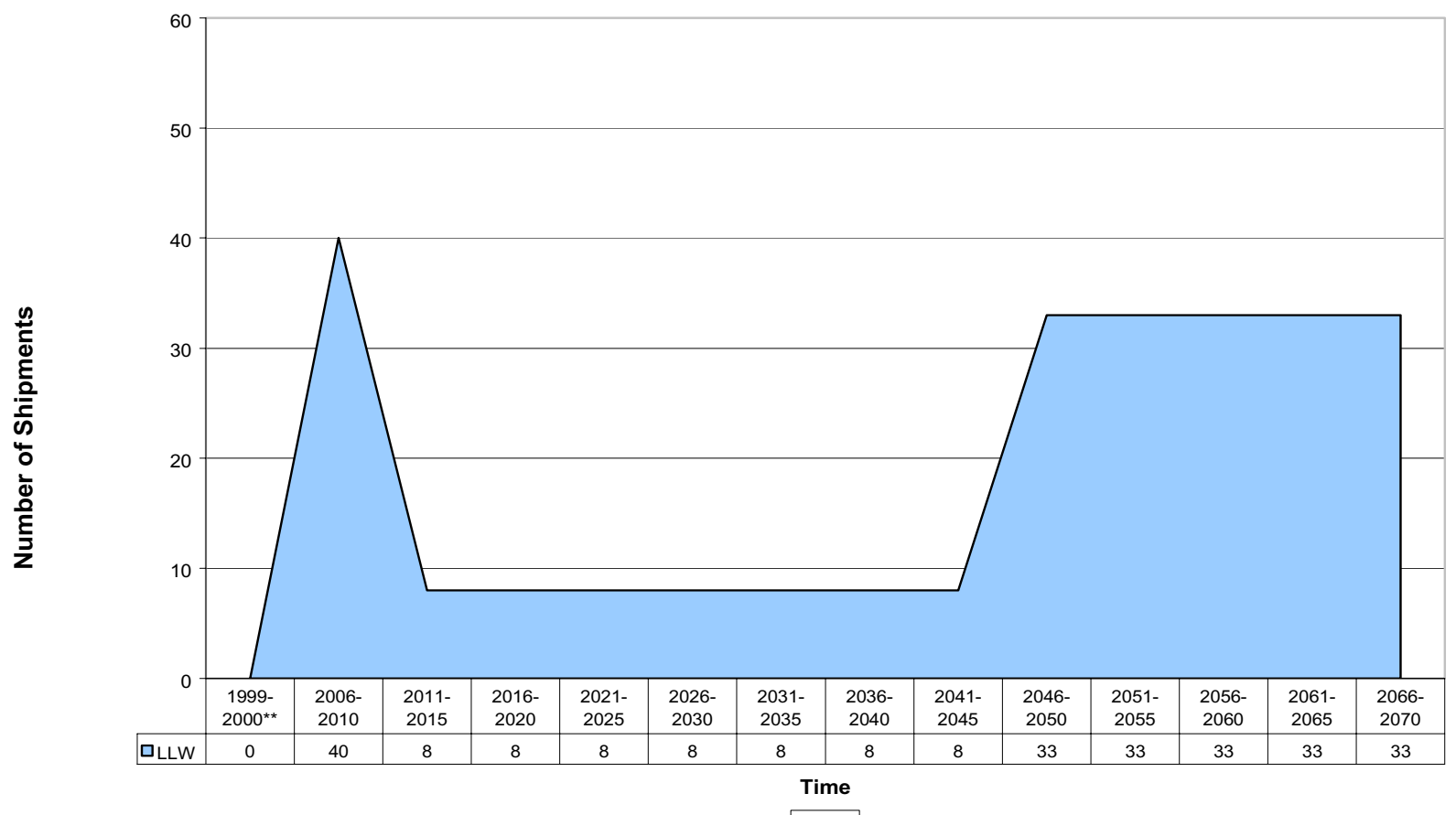

口LLW

Total life-cycle values per 7/30/99 Stream Disposition Data (SDD) (http://id.inel.gov/avs/).

** Represents data for a two year period. 
Nevada Test Site

Incoming LLW Shipments from "TBDO"

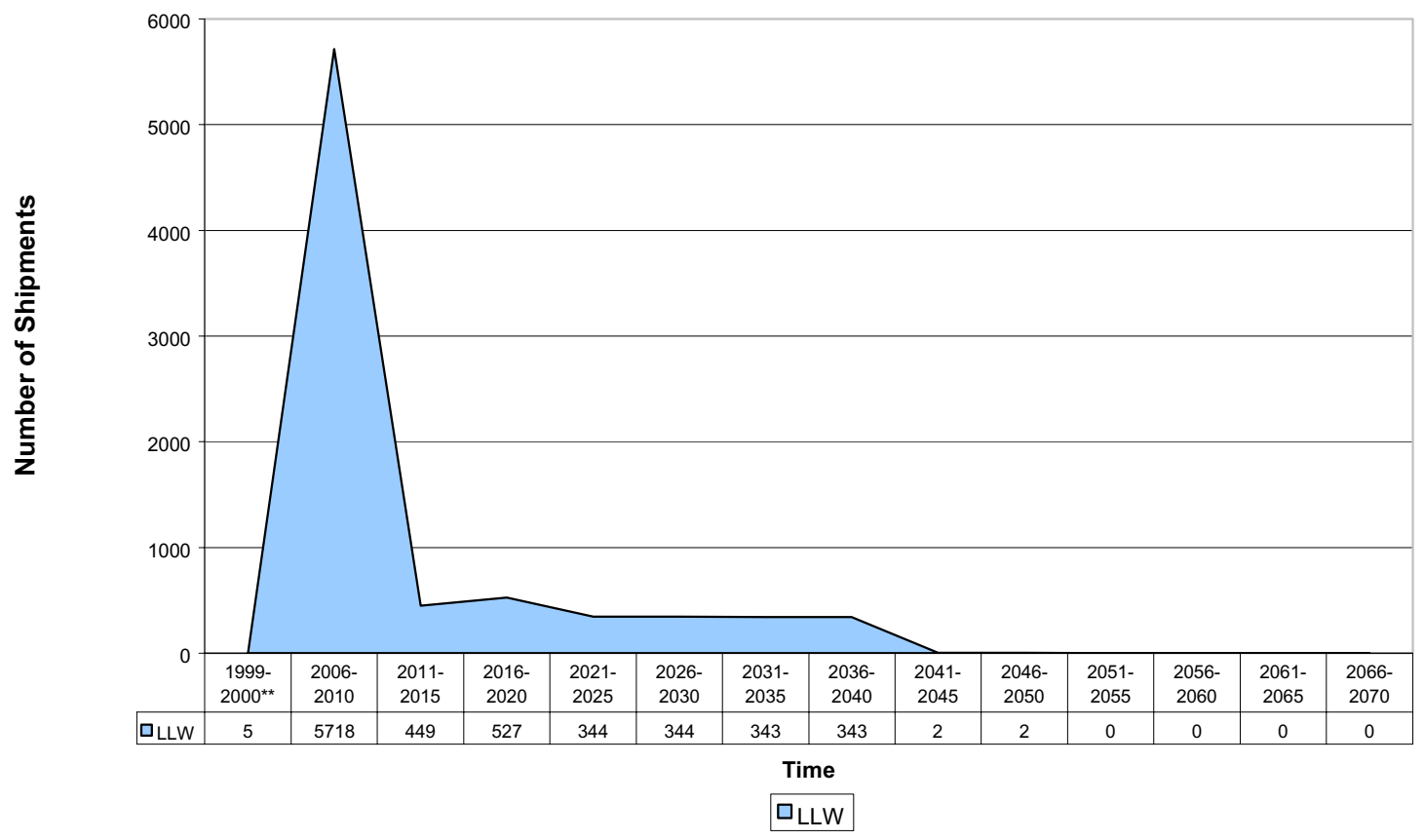

Total life-cycle values per 7/30/99 Stream Disposition Data (SDD) (http://id.inel.gov/avs/).

** Represents data for a two year period. 



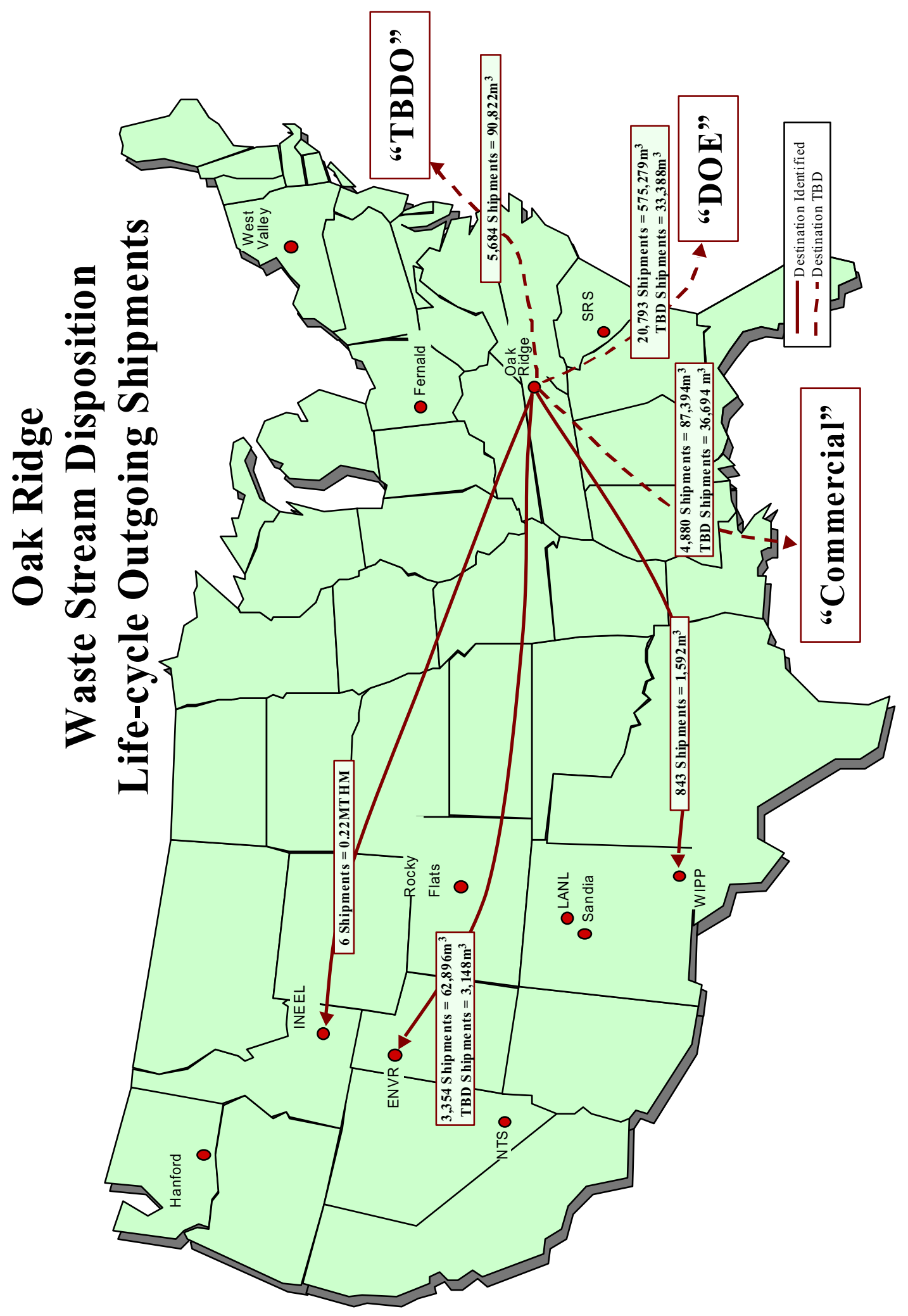

$\hat{6}$
1 
Oak Ridge

Waste Stream Disposition Life-cycle Outgoing Shipments

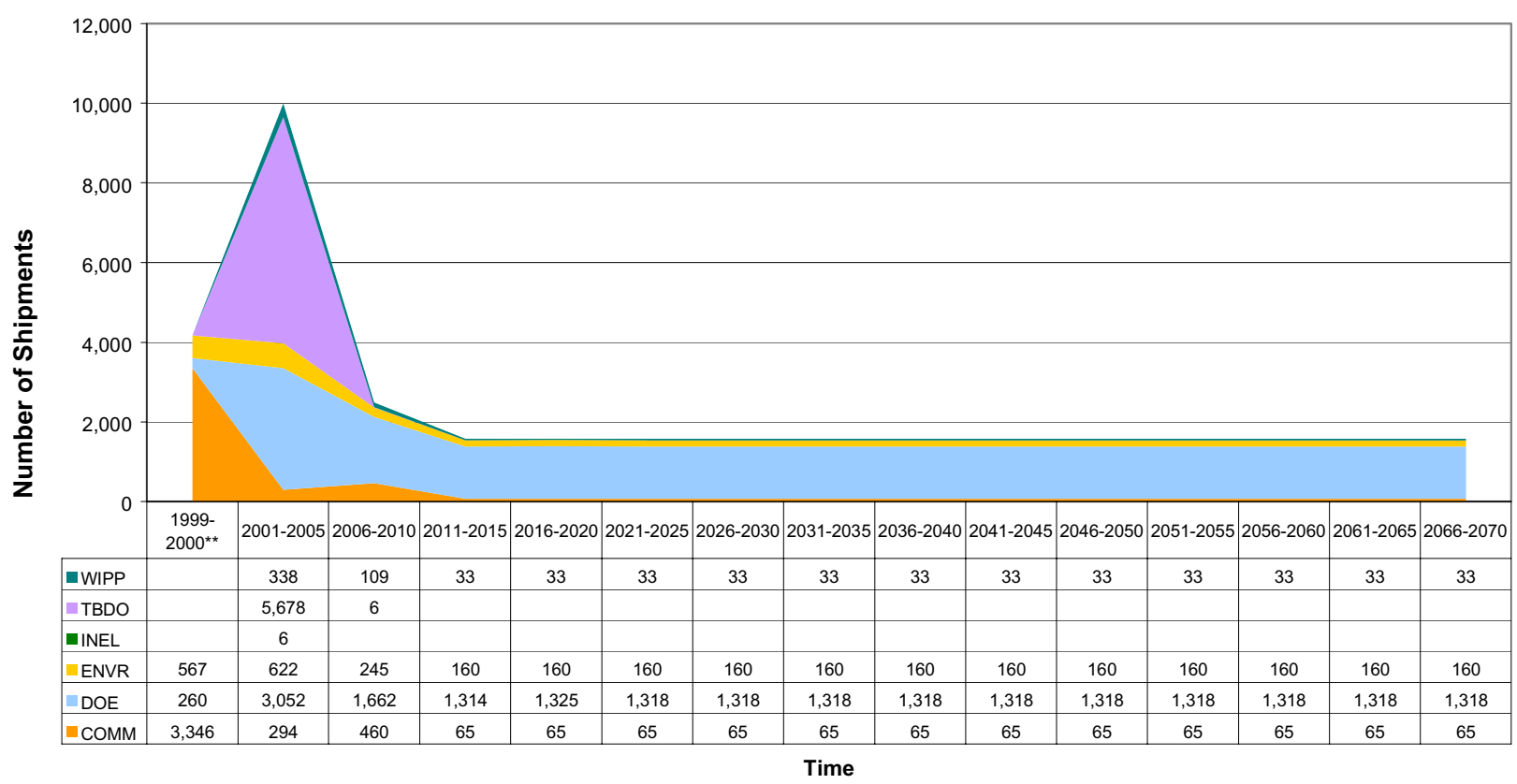

$\square \mathrm{COMM}=\mathrm{DOE} \backsim \mathrm{ENVR} \backsim \mathrm{INEL} \backsim \mathrm{TBDO} \backsim \mathrm{WIPP}$

Oak Ridge

Outgoing SNF Shipments to INEEL

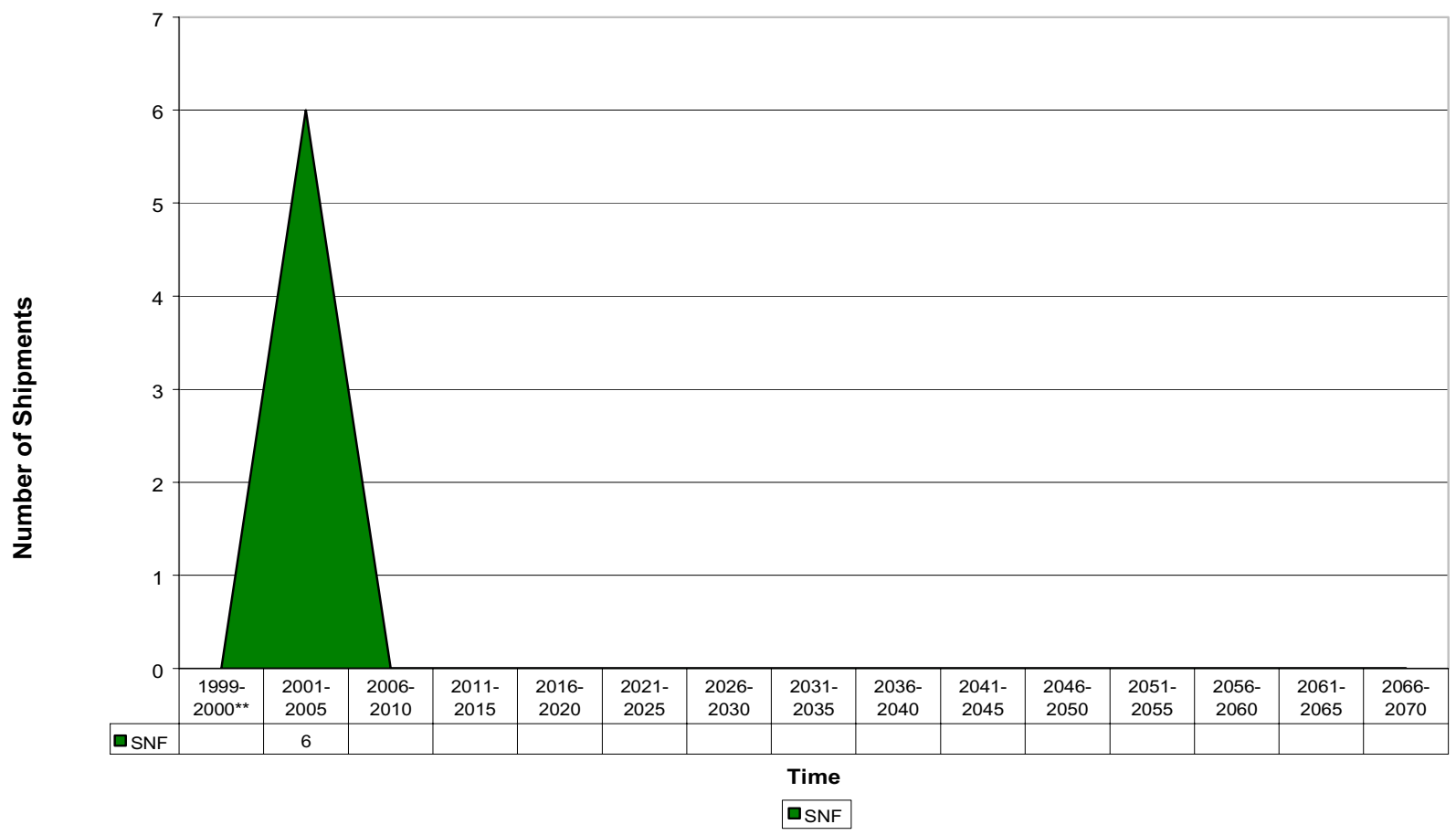

Total life-cycle values per 7/30/99 Stream Disposition Data (SDD) (http://id.inel.gov/avs/).

** Represents data for a two year period. 
Oak Ridge

Outgoing M/LLW Shipments to Envirocare

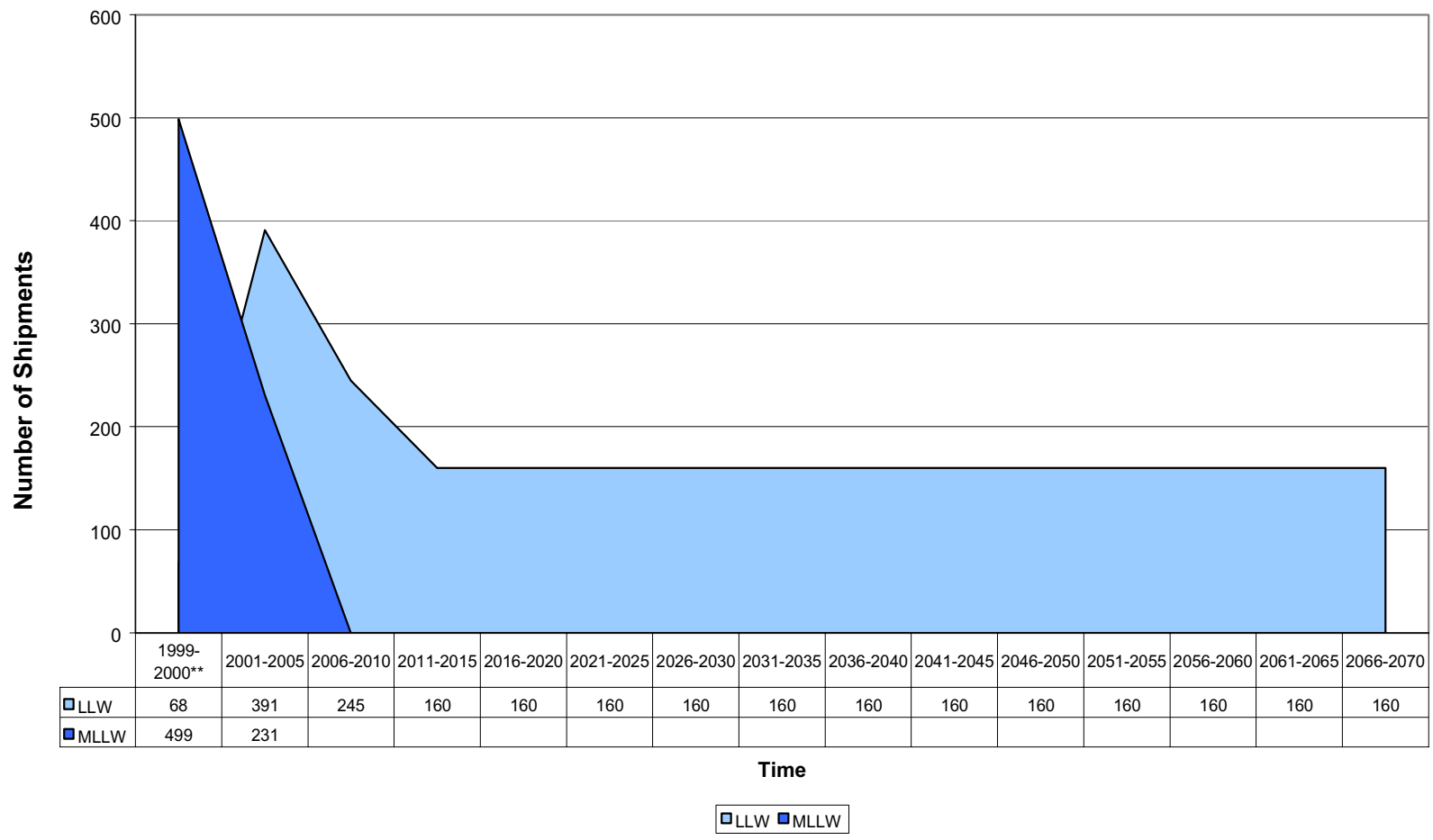

Oak Ridge

Outgoing M/LLW Shipments to WIPP

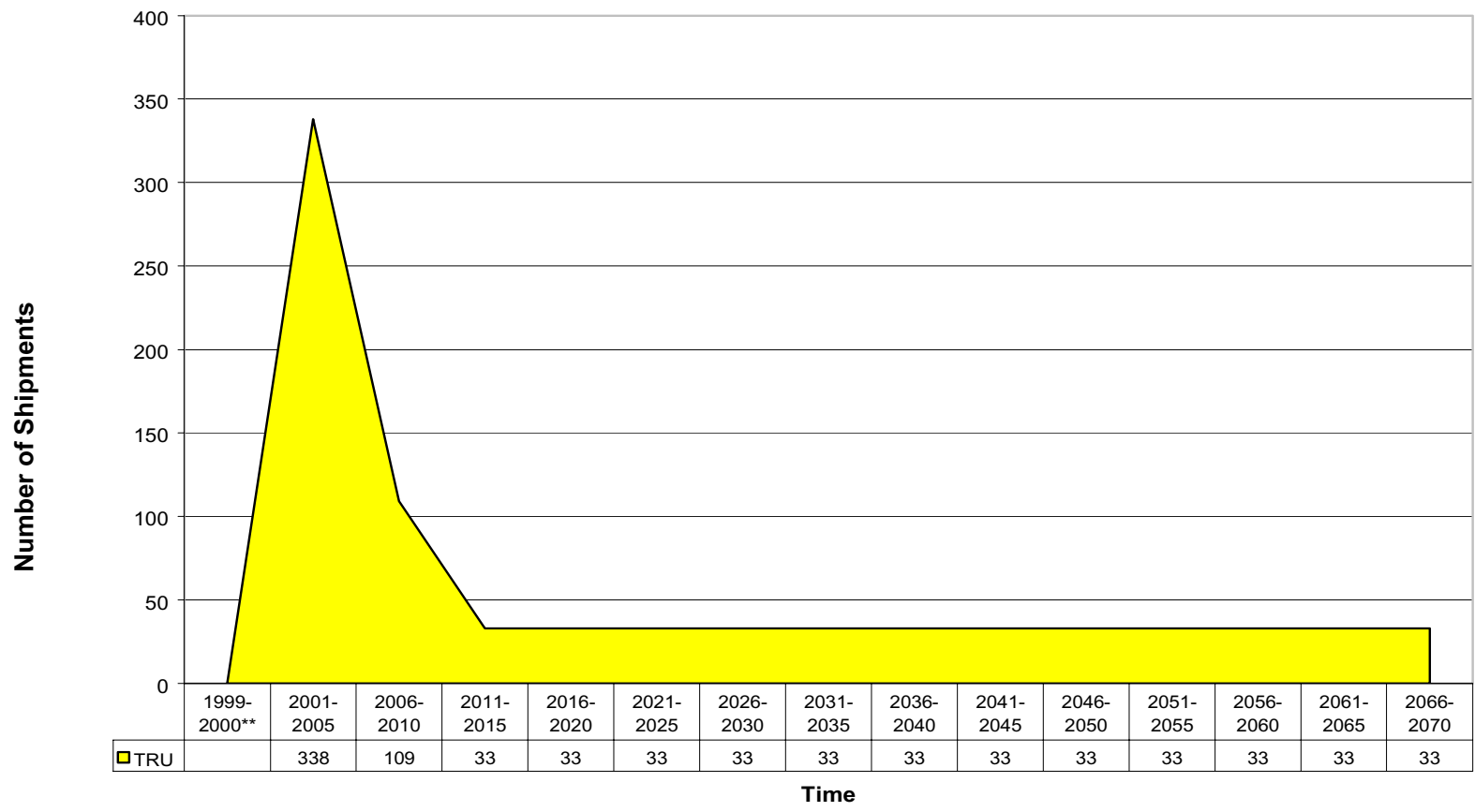

QTRU

Total life-cycle values per 7/30/99 Stream Disposition Data (SDD) (http://id.inel.gov/avs/)

** Represents data for a two year period. 
Oak Ridge

Outgoing M/LLW Shipments to "Commercial"

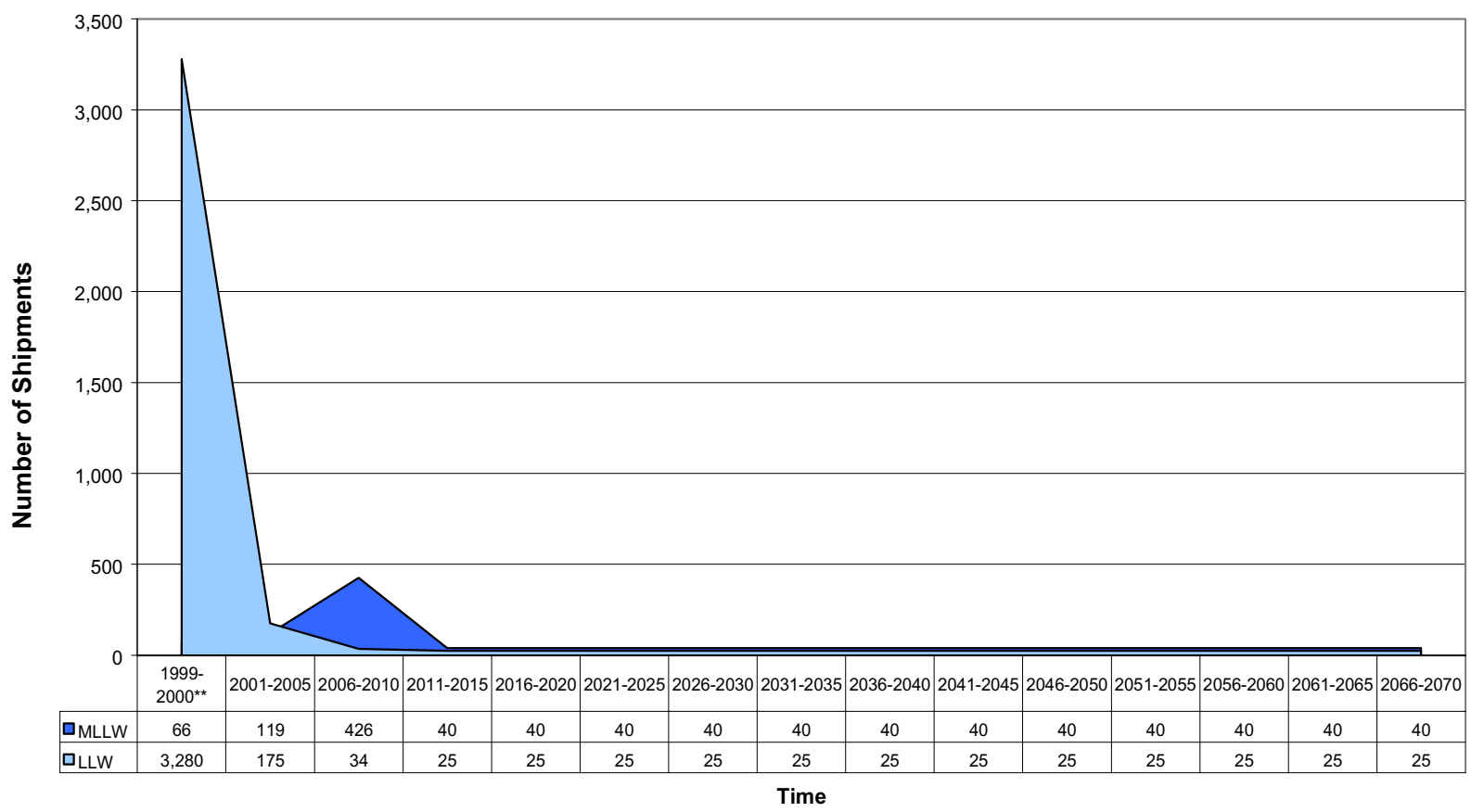

$\square_{\text {MLLW }} \square_{\text {LLW }}$

Oak Ridge

Outgoing LLW Shipments to "DOE"

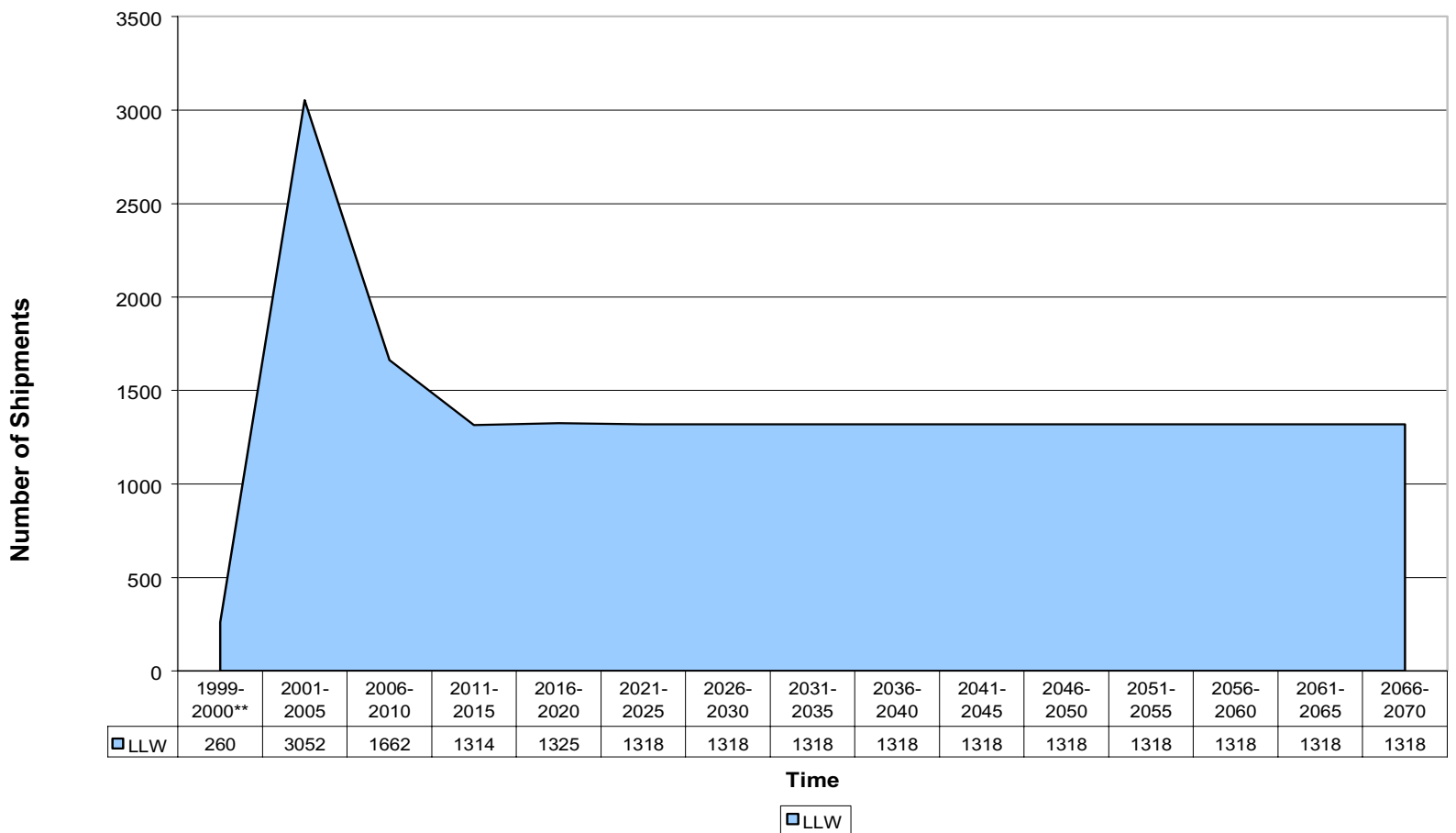

Total life-cycle values per 7/30/99 Stream Disposition Data (SDD) (http://id.inel.gov/avs/).

** Represents data for a two year period. 
Oak Ridge

Outgoing M/LLW Shipments to "TBDO"

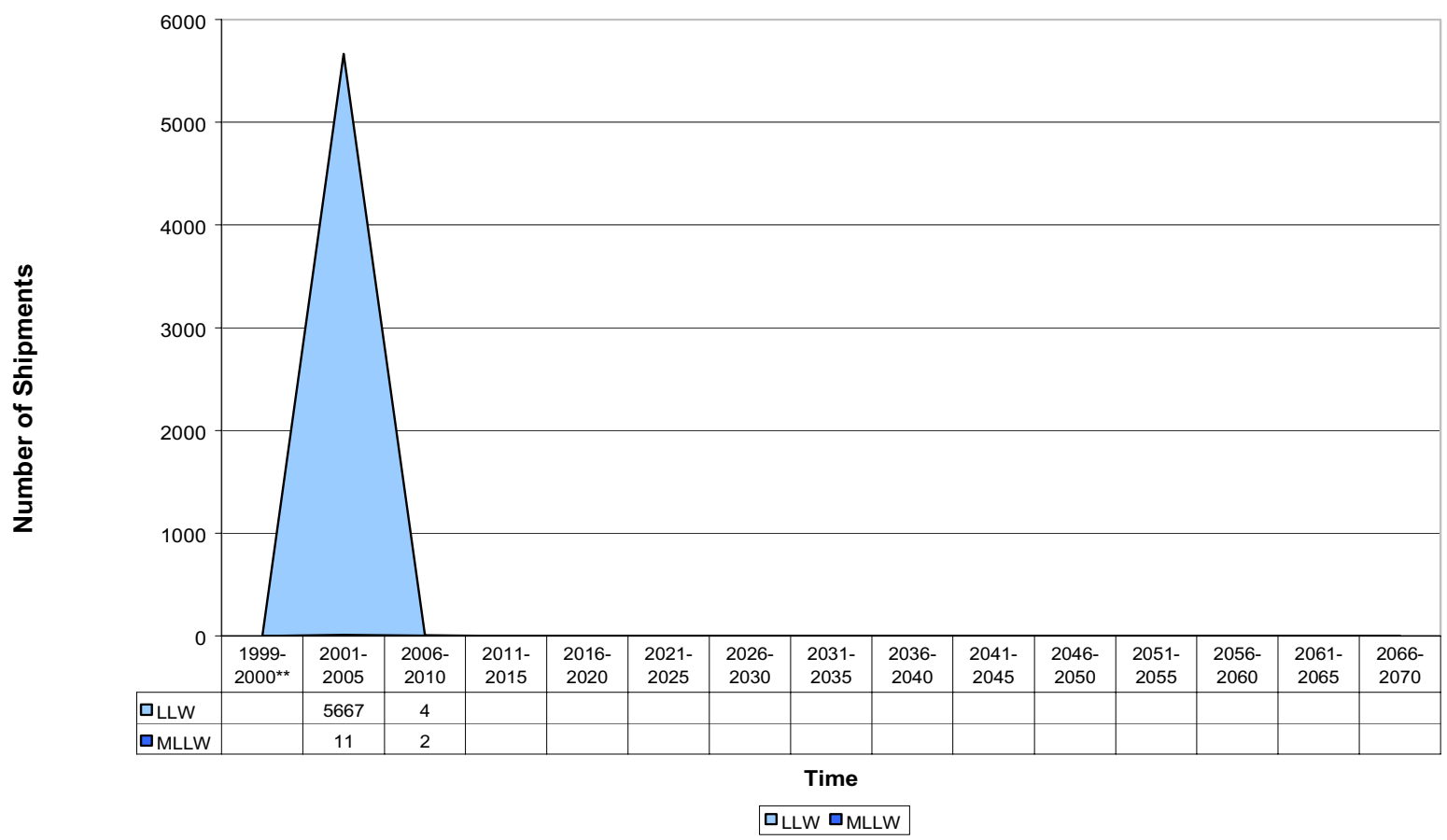

Oak Ridge

Waste Stream Disposition

Life-cycle Outgoing Volume

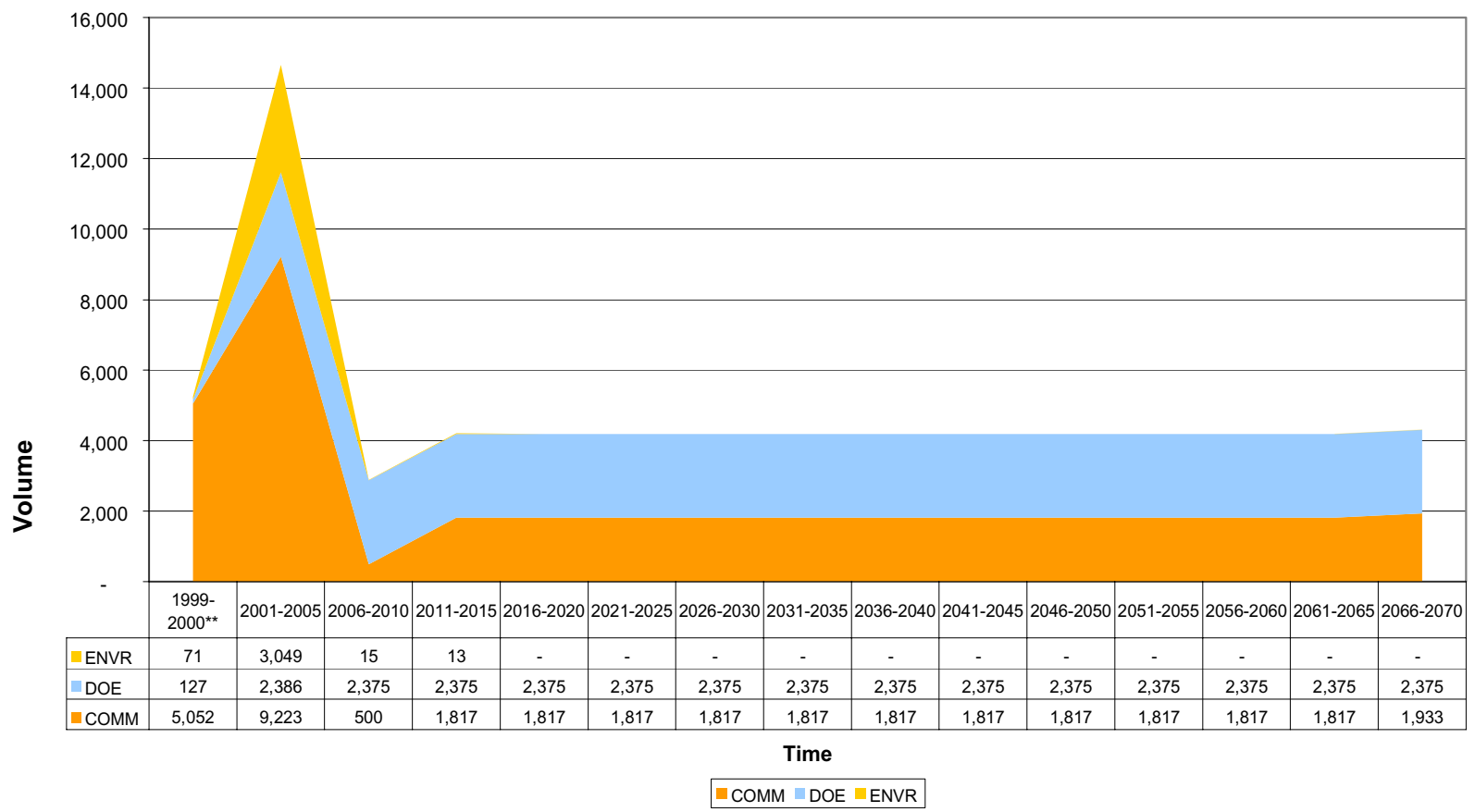

Total life-cycle values per 7/30/99 Stream Disposition Data (SDD) (http://id.inel.gov/avs/).

** Represents data for a two year period. 
Oak Ridge

Outgoing M/LLW Volume to "Commercial"

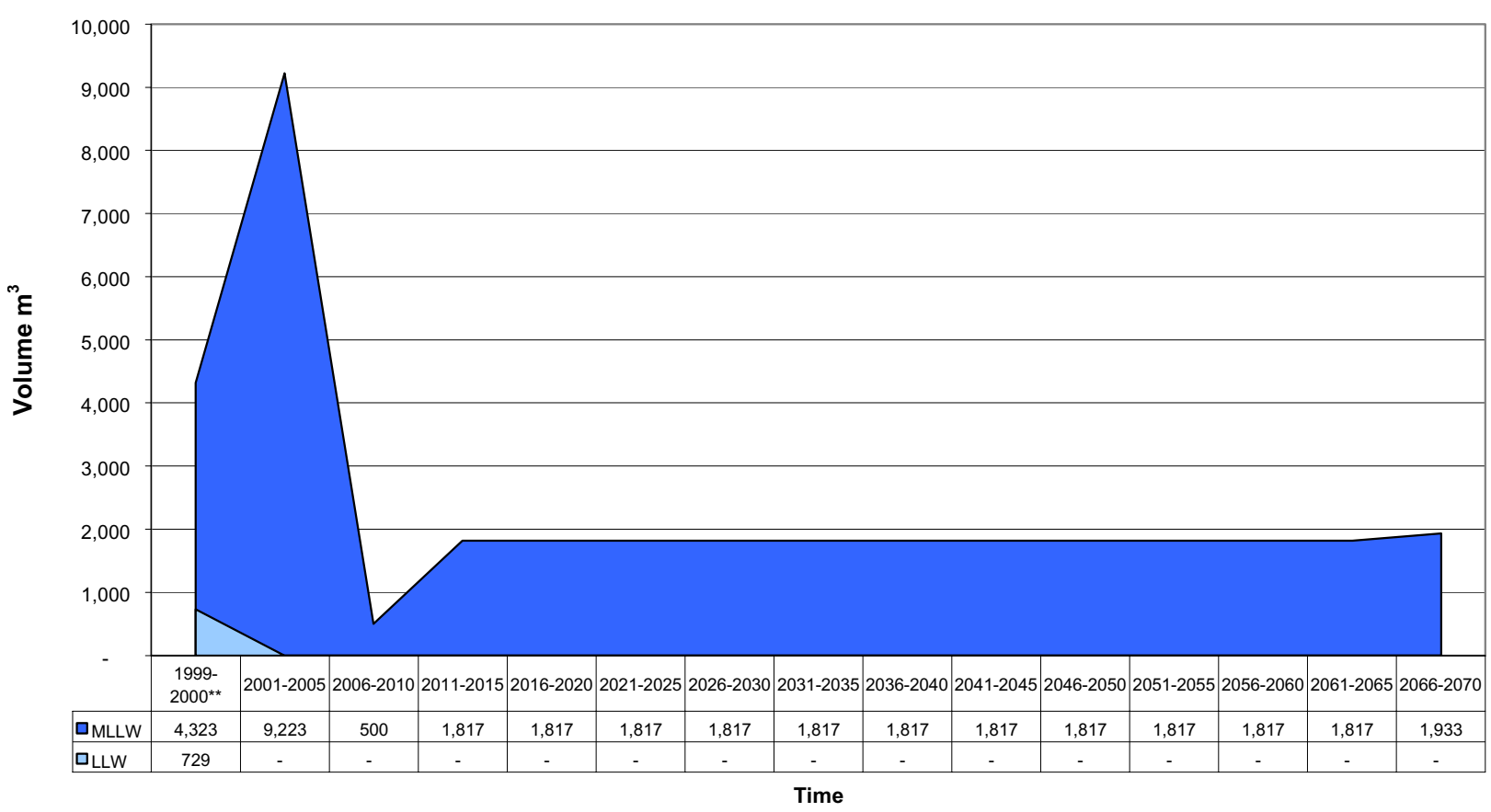

$\square$ MLLW $\square$ LLW

Oak Ridge

Outgoing LLW Volume to DOE

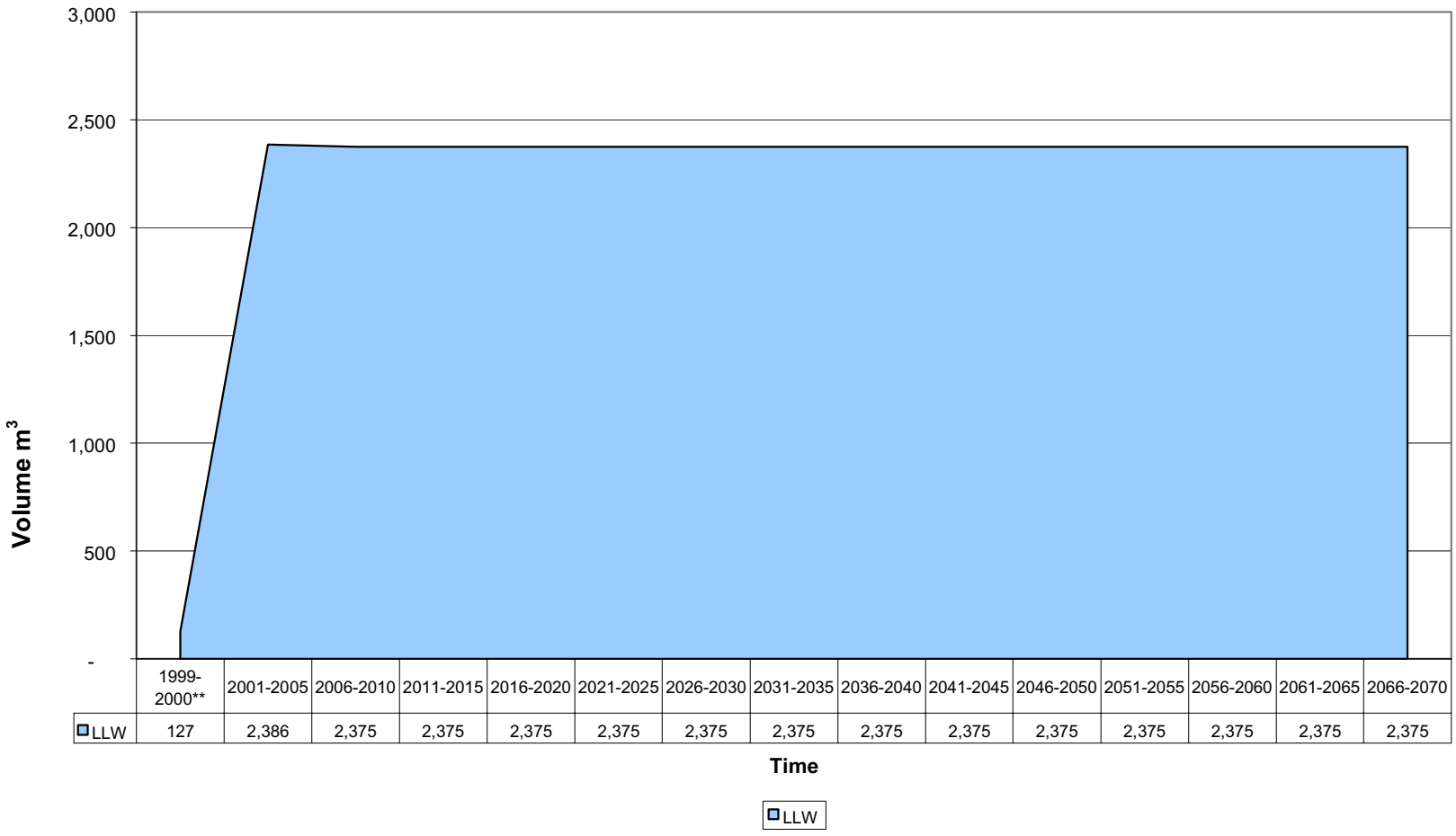

Total life-cycle values per 7/30/99 Stream Disposition Data (SDD) (http://id.inel.gov/avs/).

** Represents data for a two year period. 
Oak Ridge

Outgoing M/LLW Volume to Envirocare

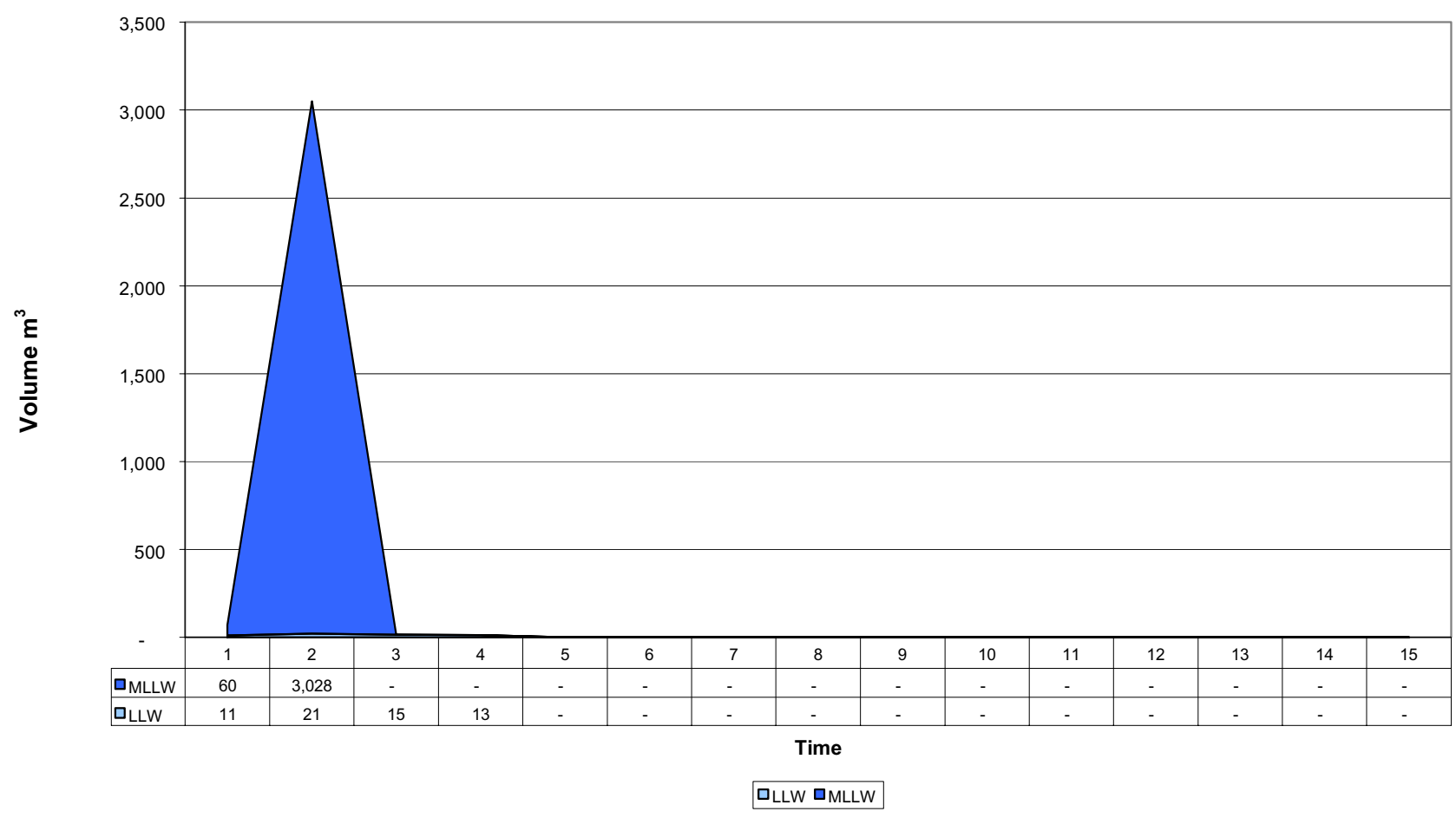

Total life-cycle values per 7/30/99 Stream Disposition Data (SDD) (http://id.inel.gov/avs/)

** Represents data for a two year period. 



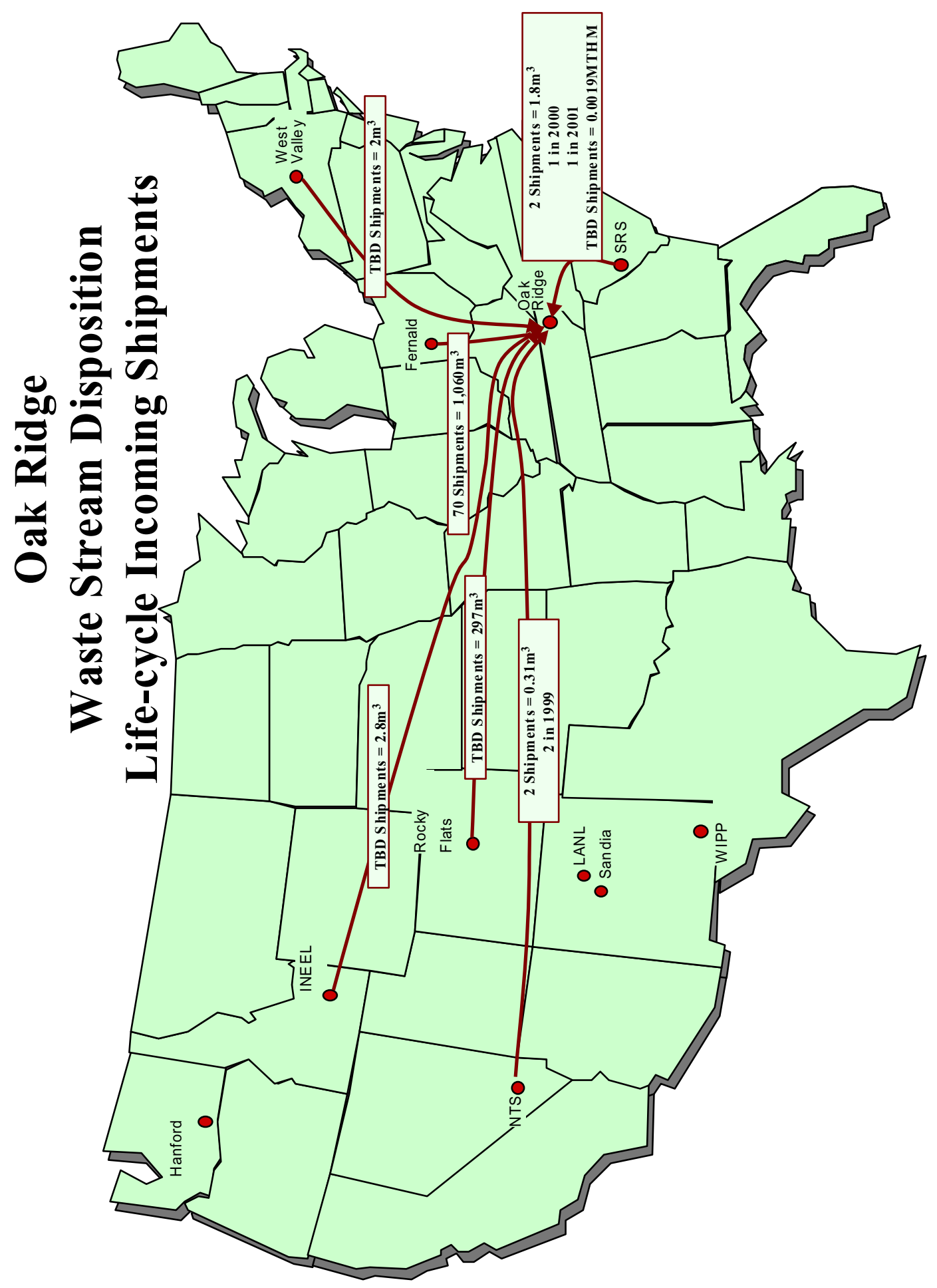


Oak Ridge

Waste Stream Disposition

Life-cycle Incoming Shipments

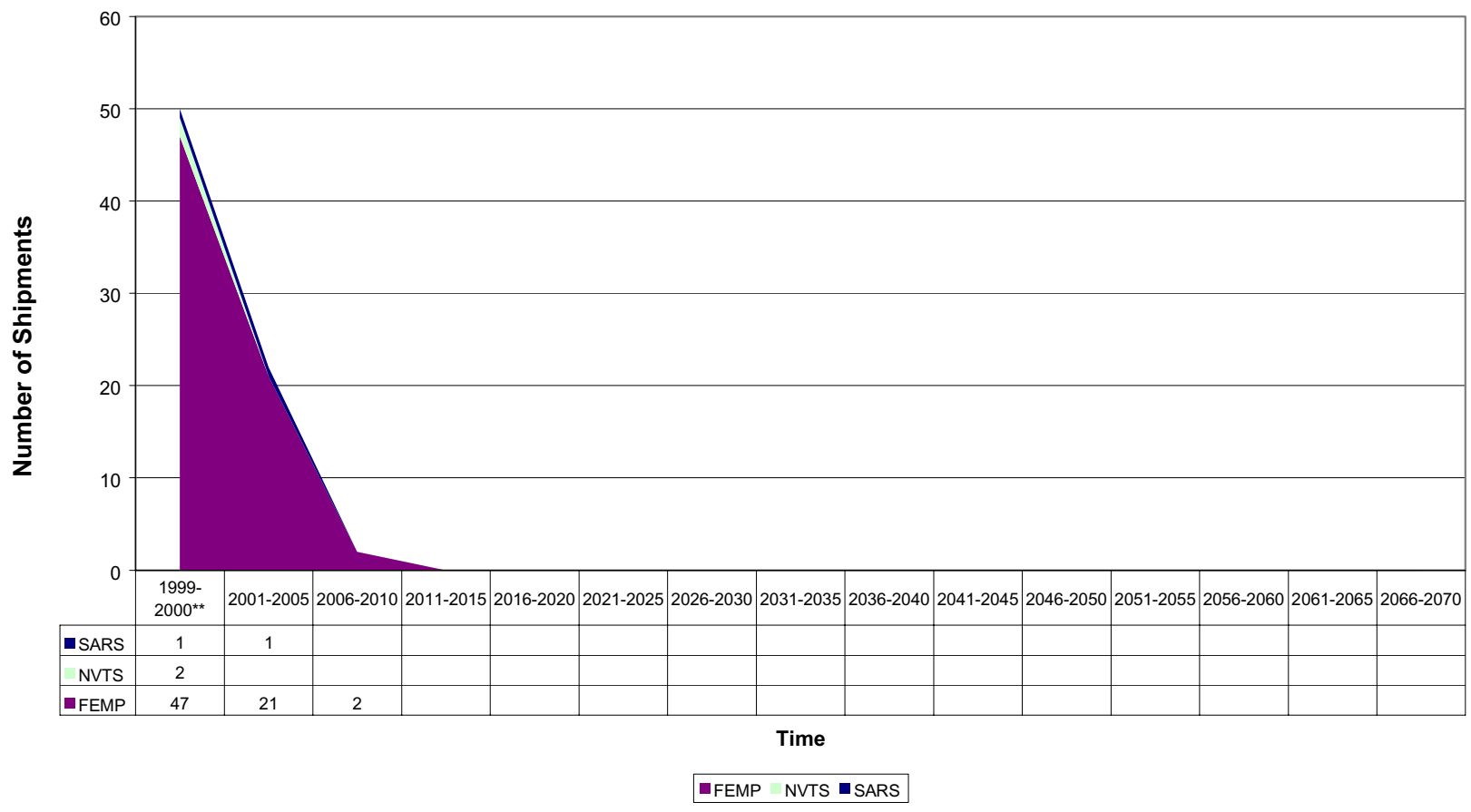

Oak Ridge

Incoming MLLW Shipments from Fernald

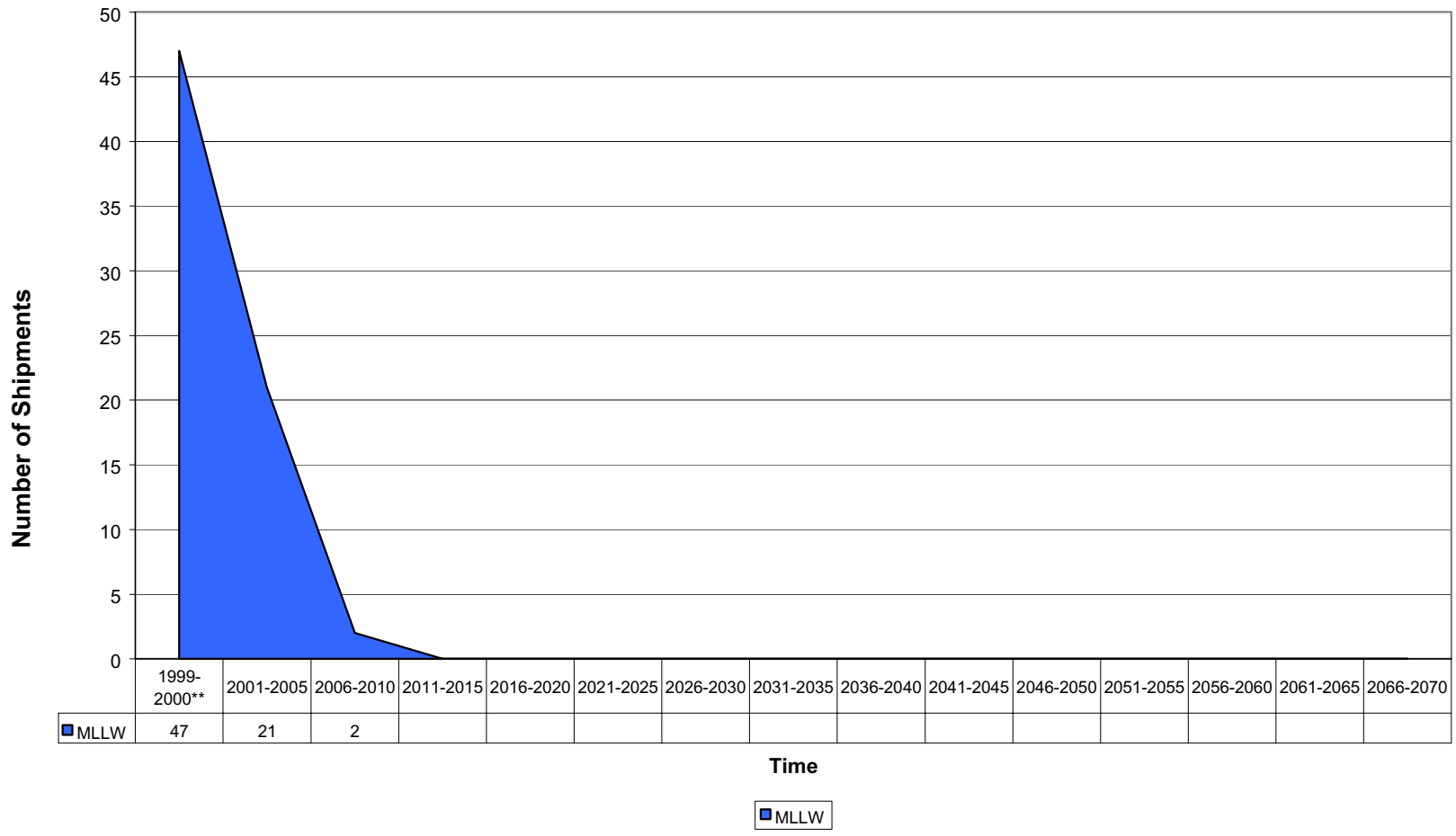

Total life-cycle values per 7/30/99 Stream Disposition Data (SDD) (http://id.inel.gov/avs/).

** Represents data for a two year period. 
Oak Ridge

Waste Stream Disposition Life-cycle Incoming Volume

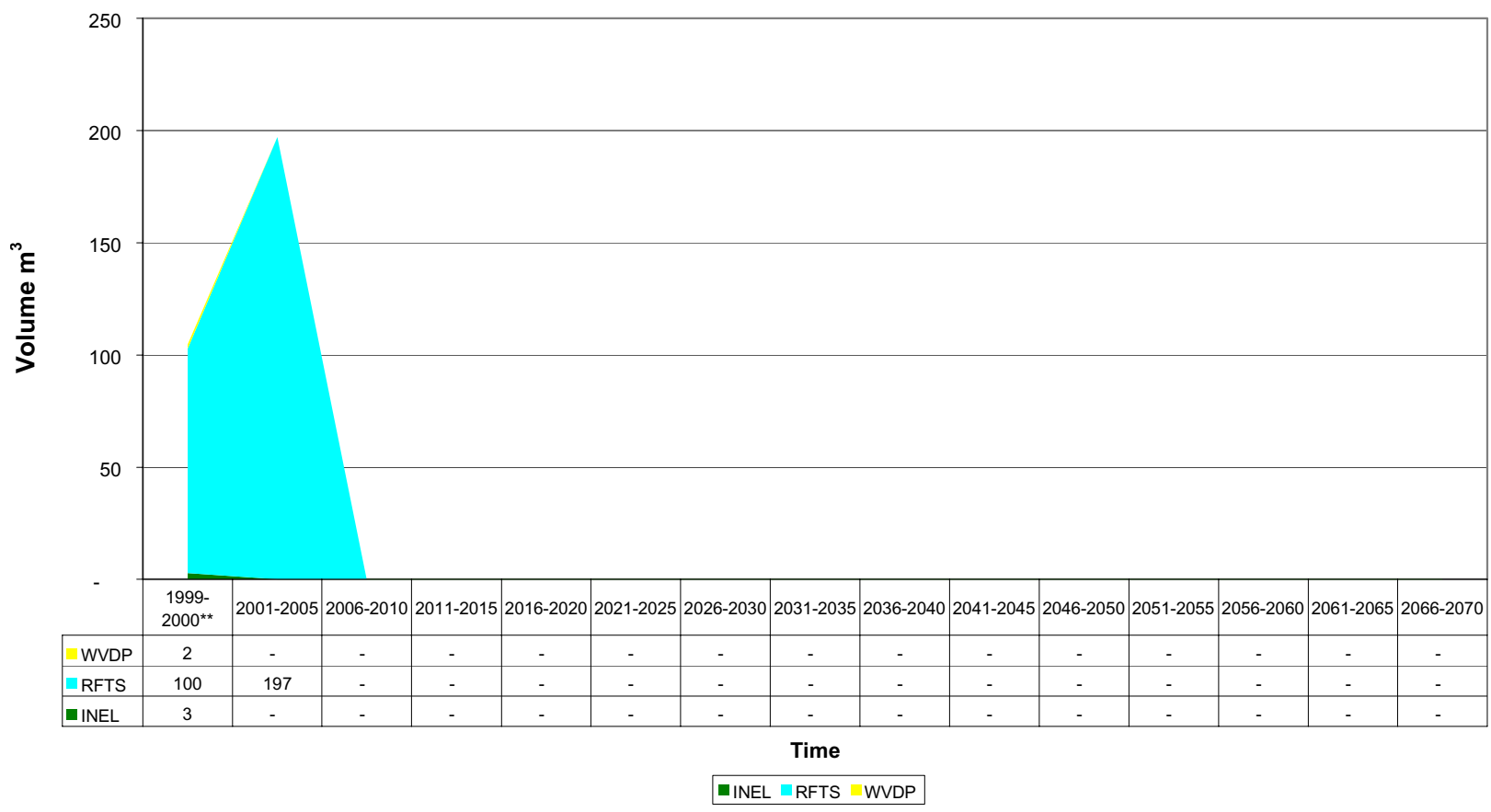

Oak Ridge

Incoming MLLW Volume from INEEL

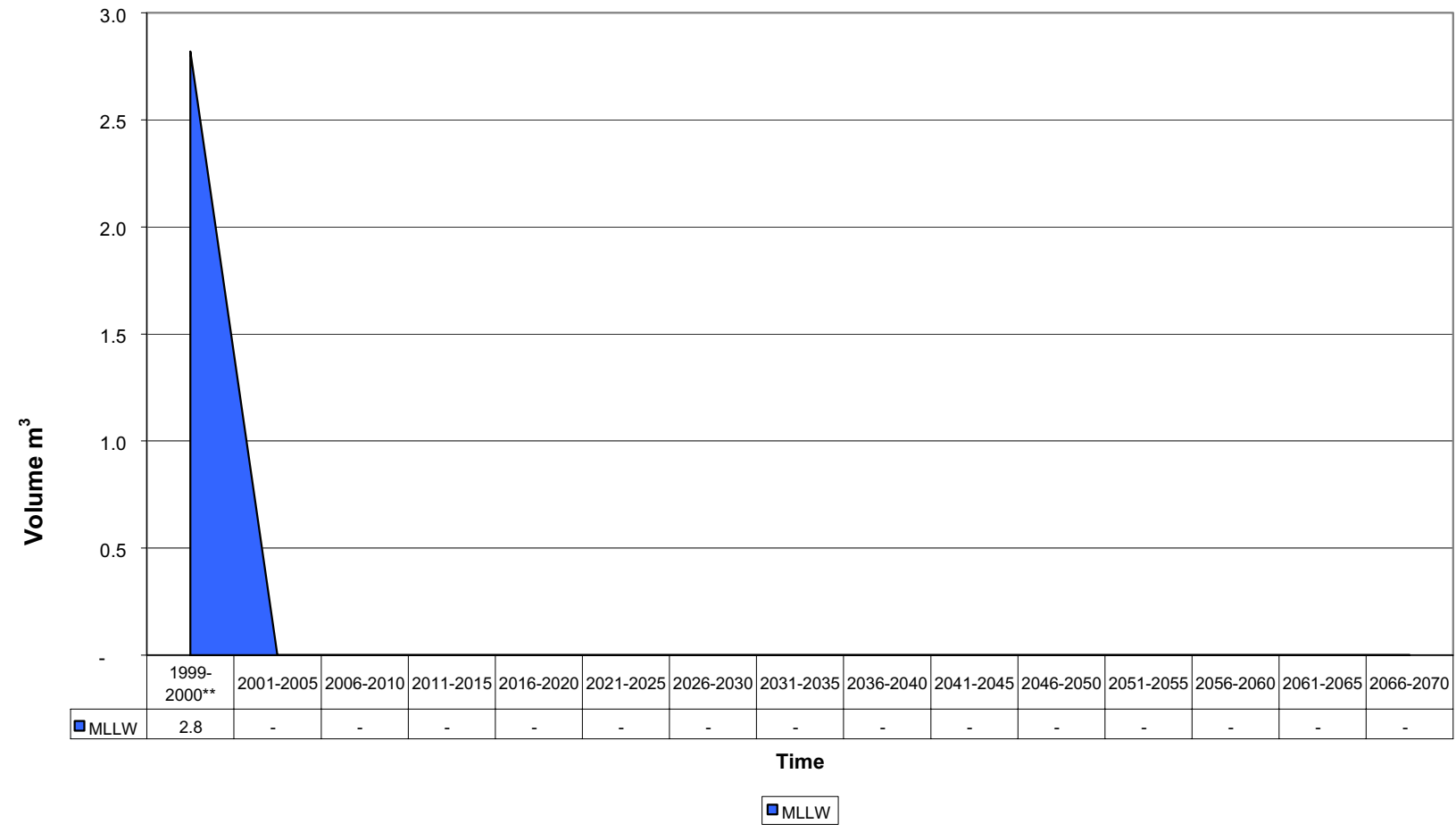

Total life-cycle values per 7/30/99 Stream Disposition Data (SDD) (http://id.inel.gov/avs/).

** Represents data for a two year period. 
Oak Ridge

Incoming M/LLW Volume from Rocky Flats

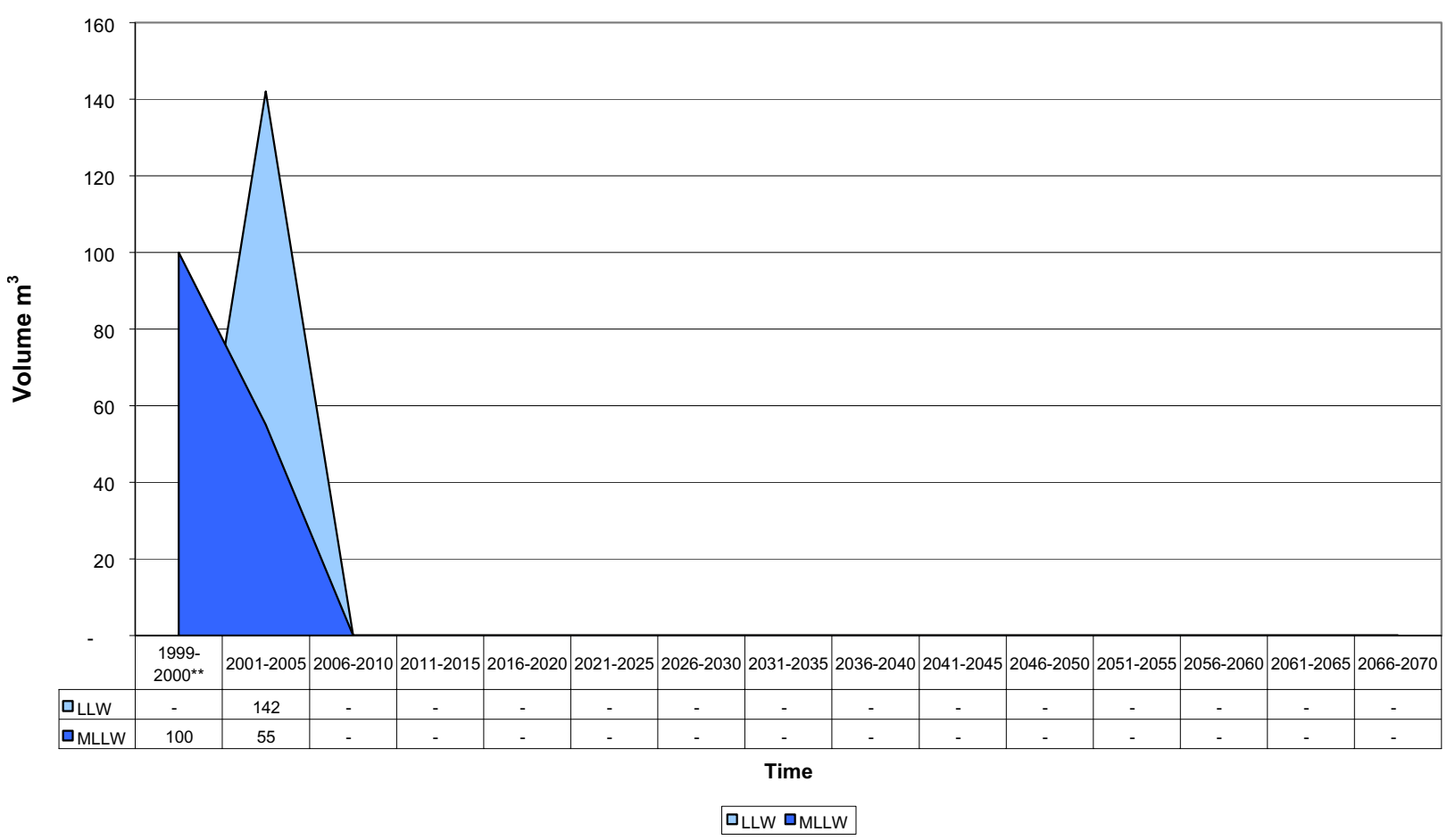

Oak Ridge

Incoming MLLW Volume from West Valley

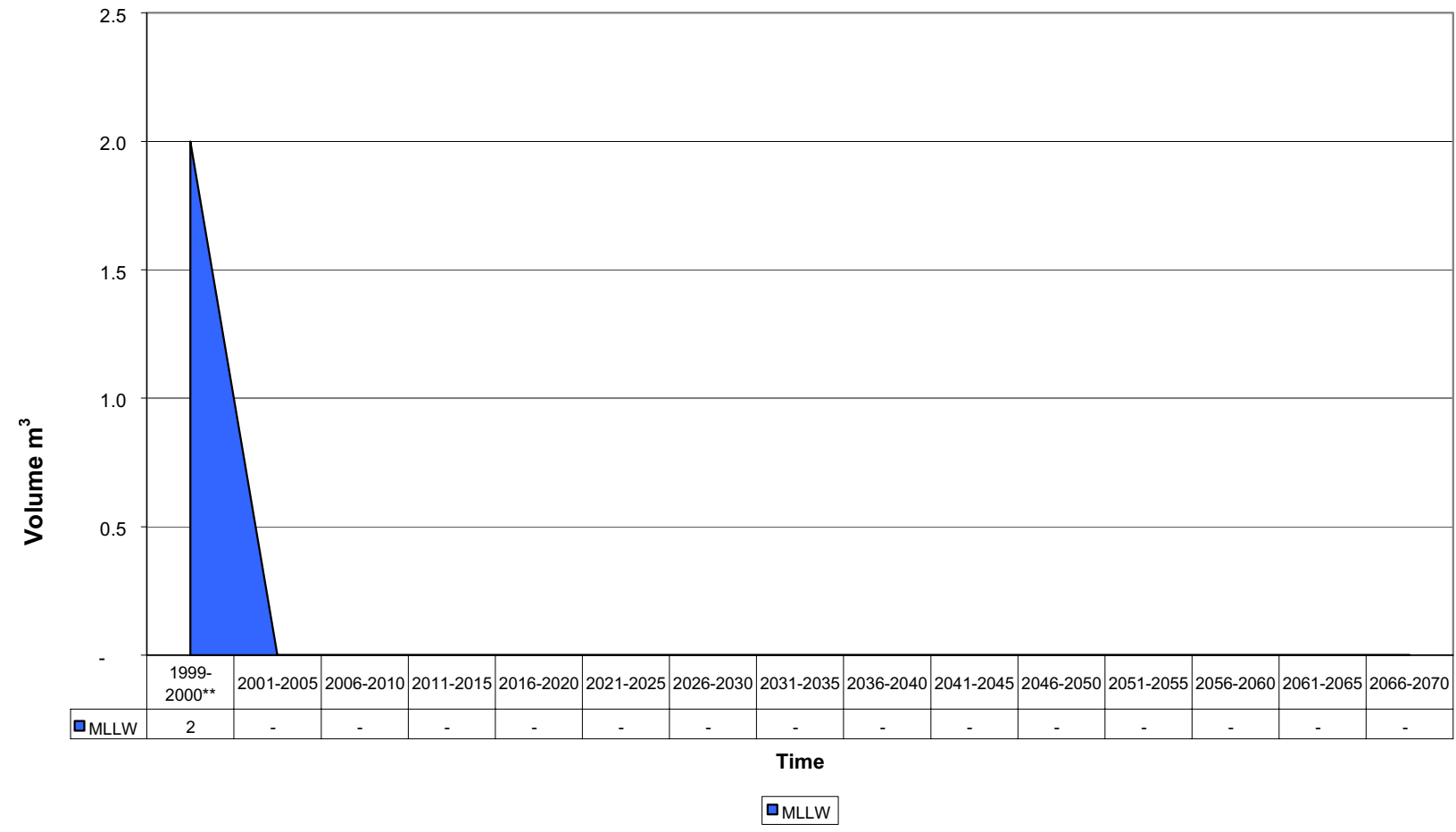

Total life-cycle values per 7/30/99 Stream Disposition Data (SDD) (http://id.inel.gov/avs/).

** Represents data for a two year period. 
Oak Ridge

Waste Stream Disposition

Life-cycle Incoming SNF

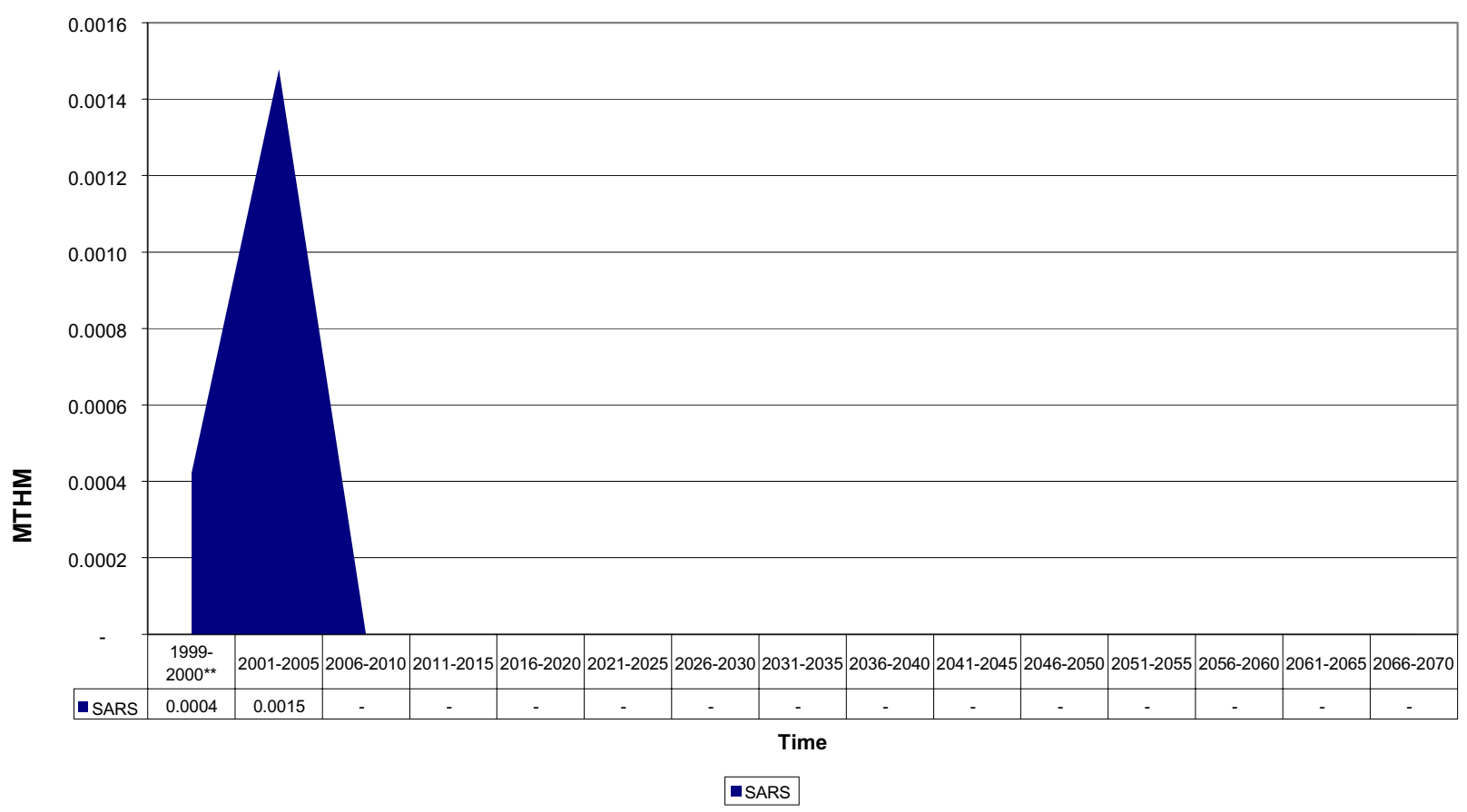

Oak Ridge

Incoming SNF from Savannah River Site

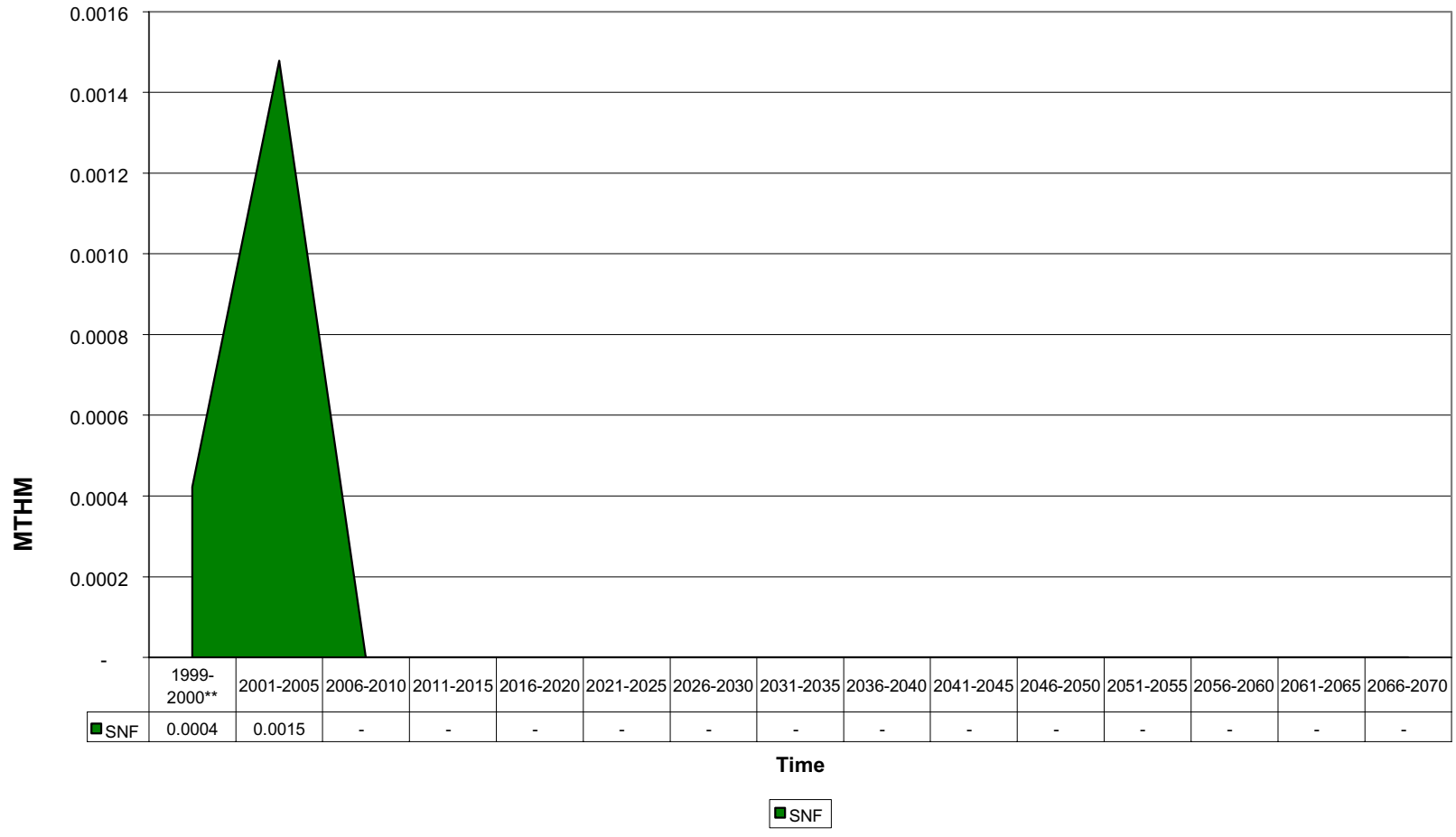

Total life-cycle values per 7/30/99 Stream Disposition Data (SDD) (http://id.inel.gov/avs/).

A-79

Printed: March 30, 2000

** Represents data for a two year period. 



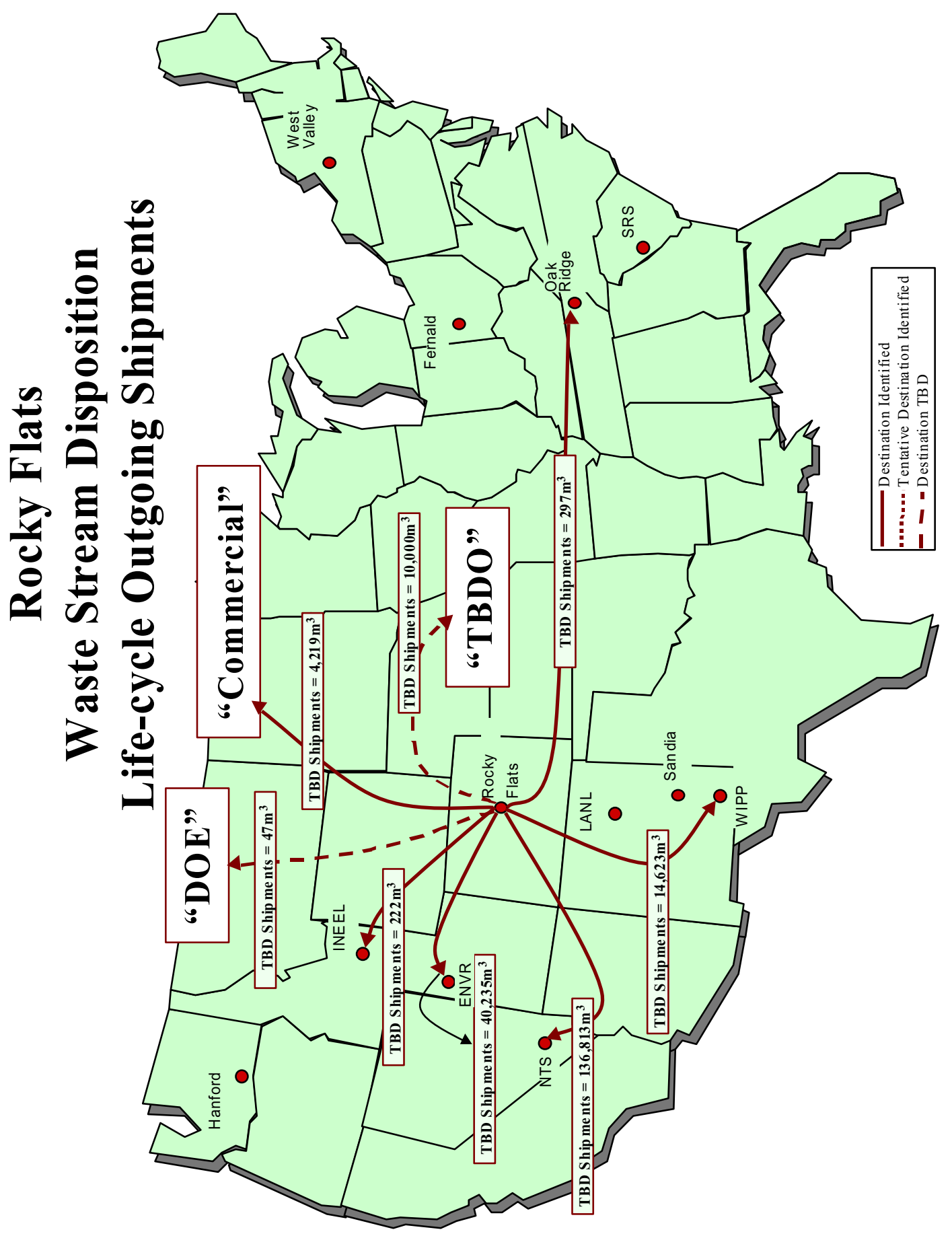


Rocky Flats

Waste Stream Disposition

Life-cycle Outgoing Volume

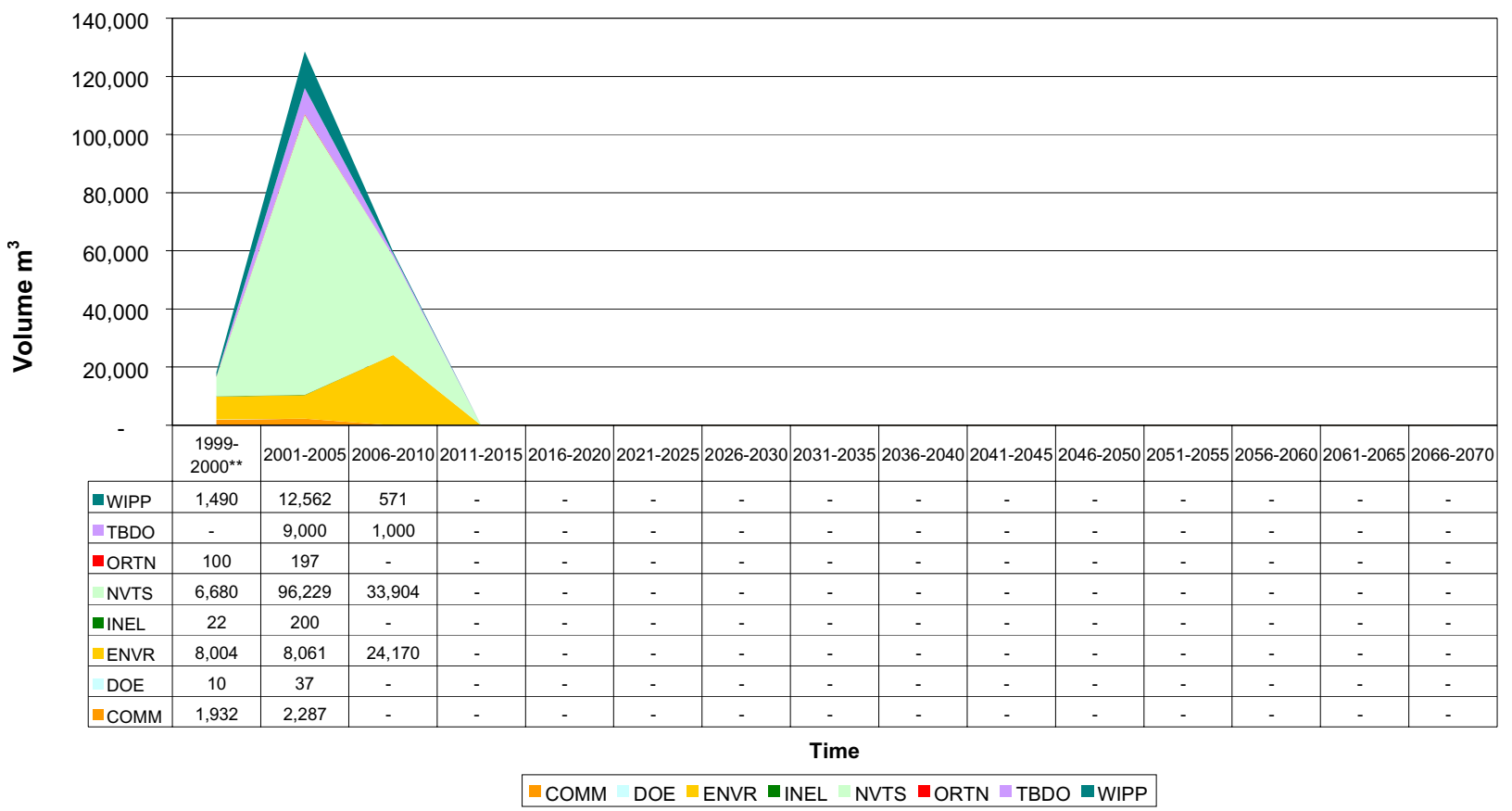

Rocky Flats

Outgoing M/LLW Volume to "Commercial"

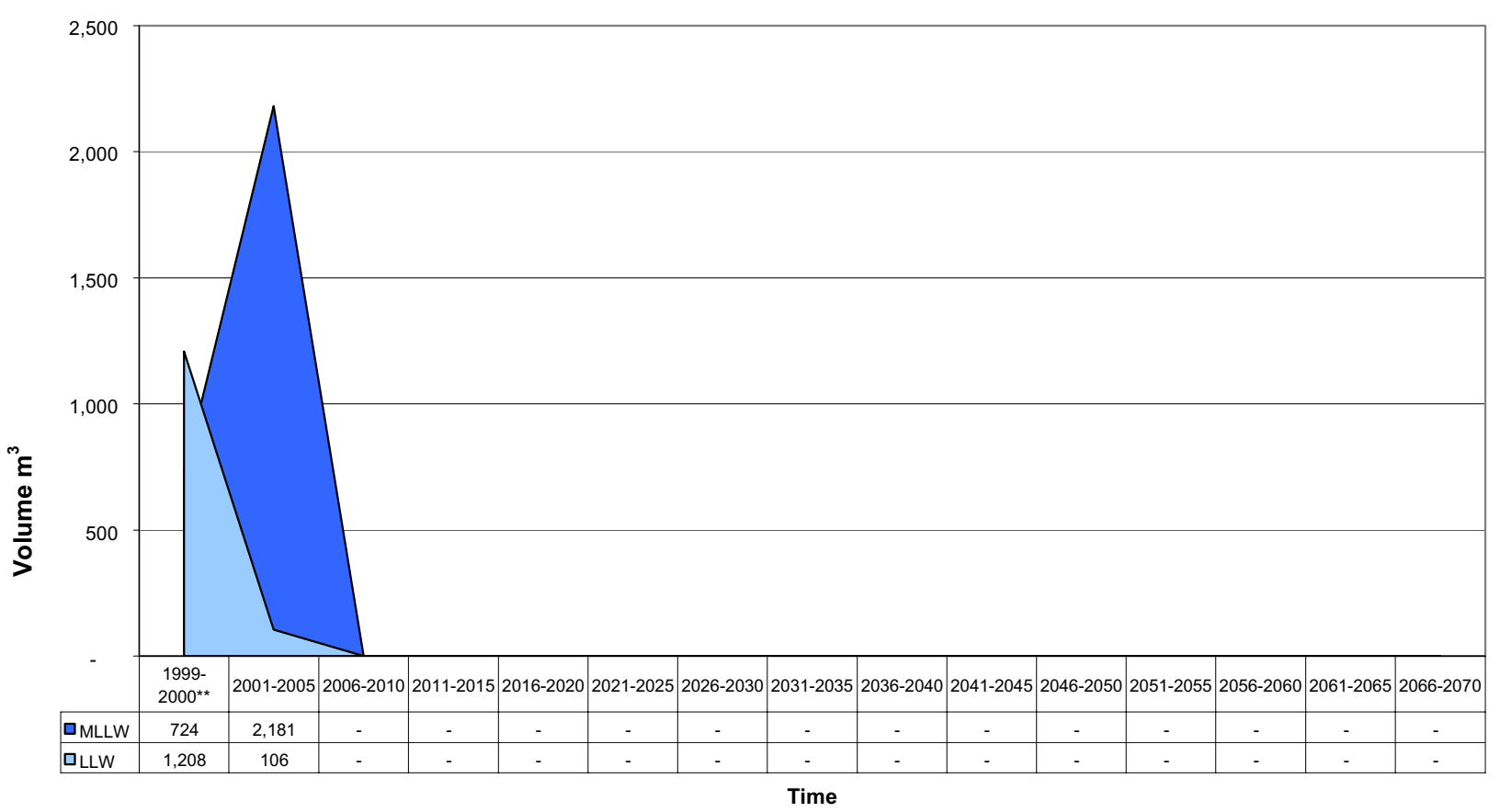

口MLLW DLLW 
Rocky Flats

Outgoing TRU Volume to "DOE"

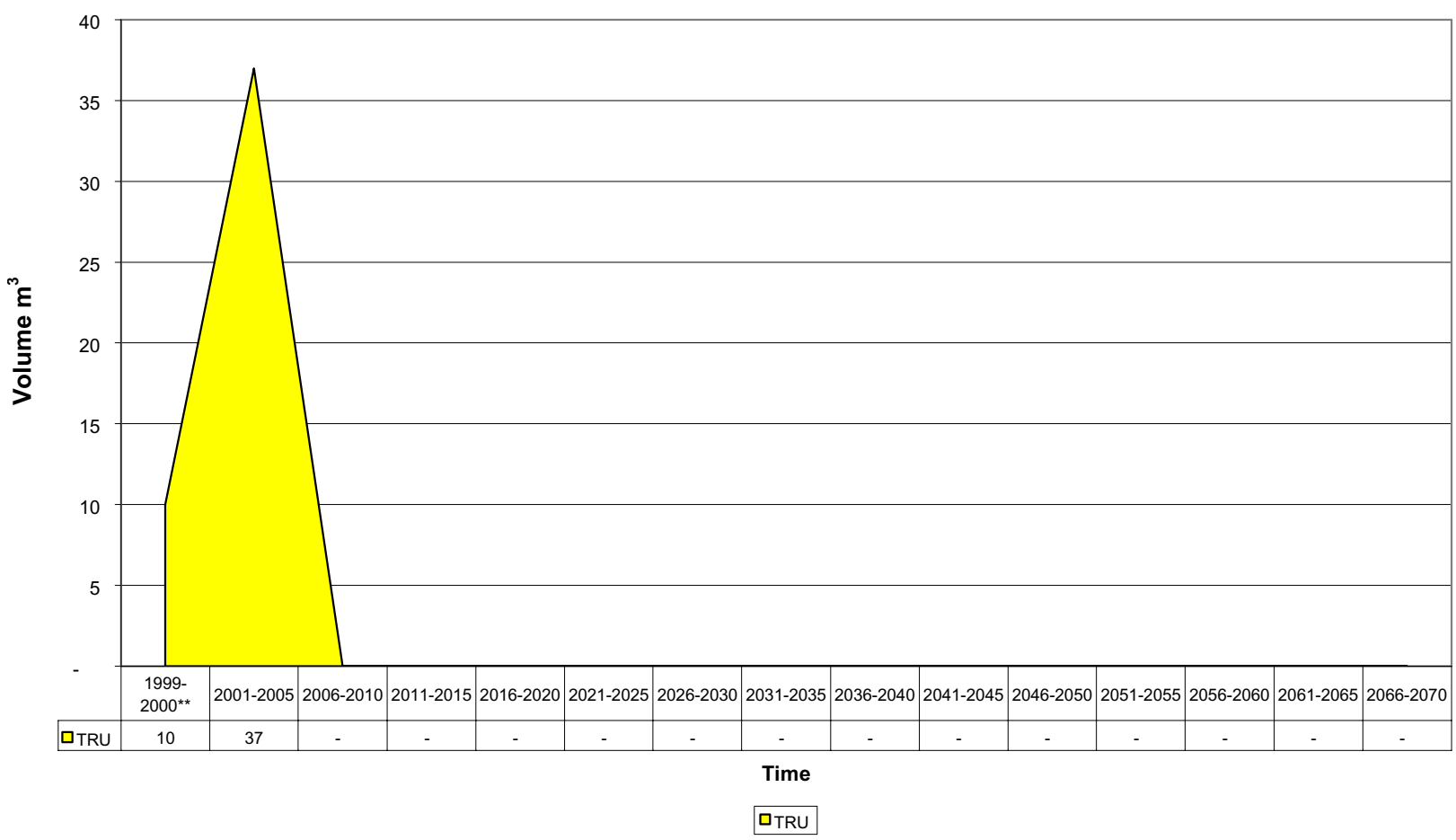

Rocky Flats

Outgoing MLLW Volume to Envirocare

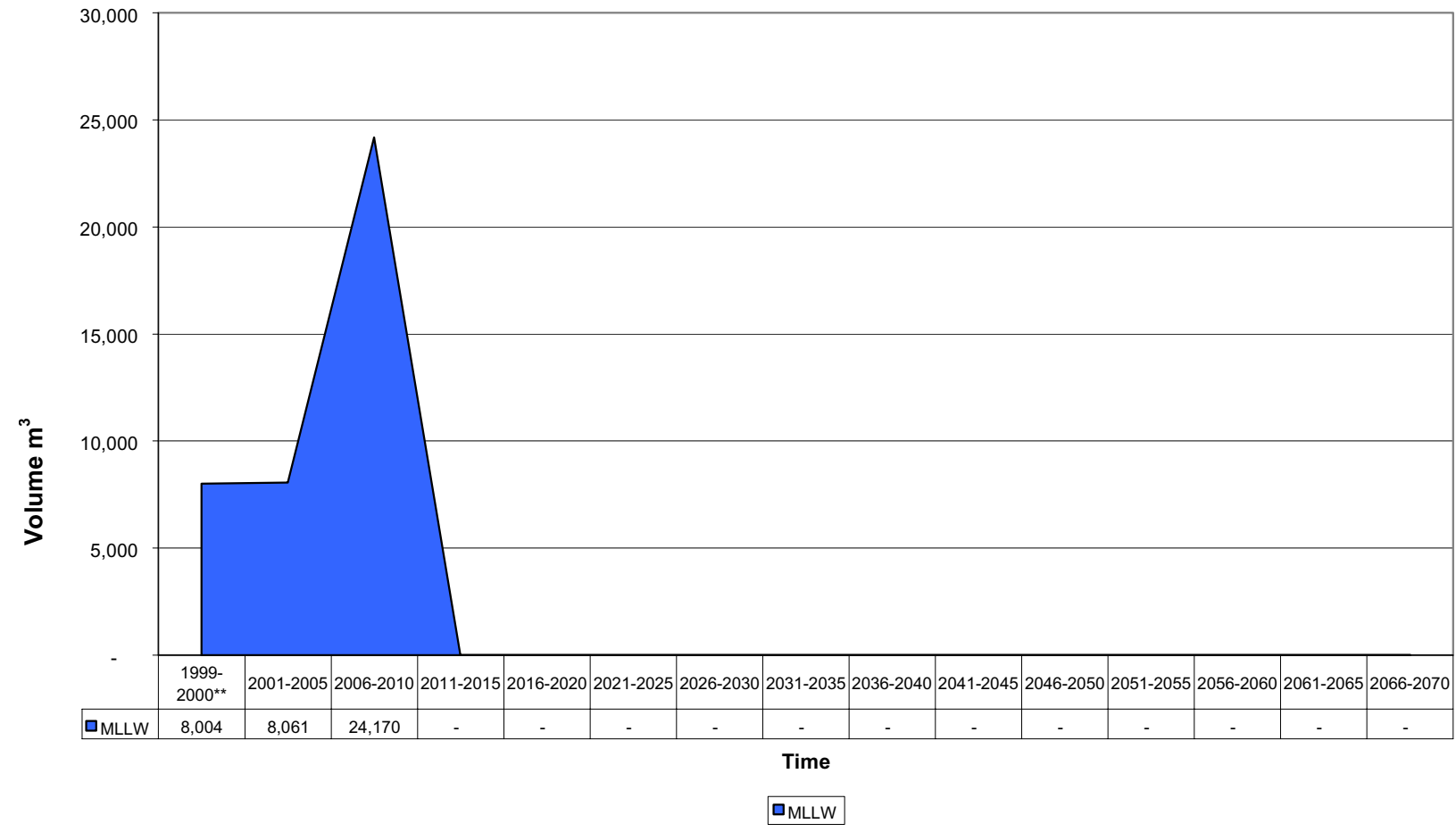


Rocky Flats

Outgoing MLLW \& TRU Volume to INEEL

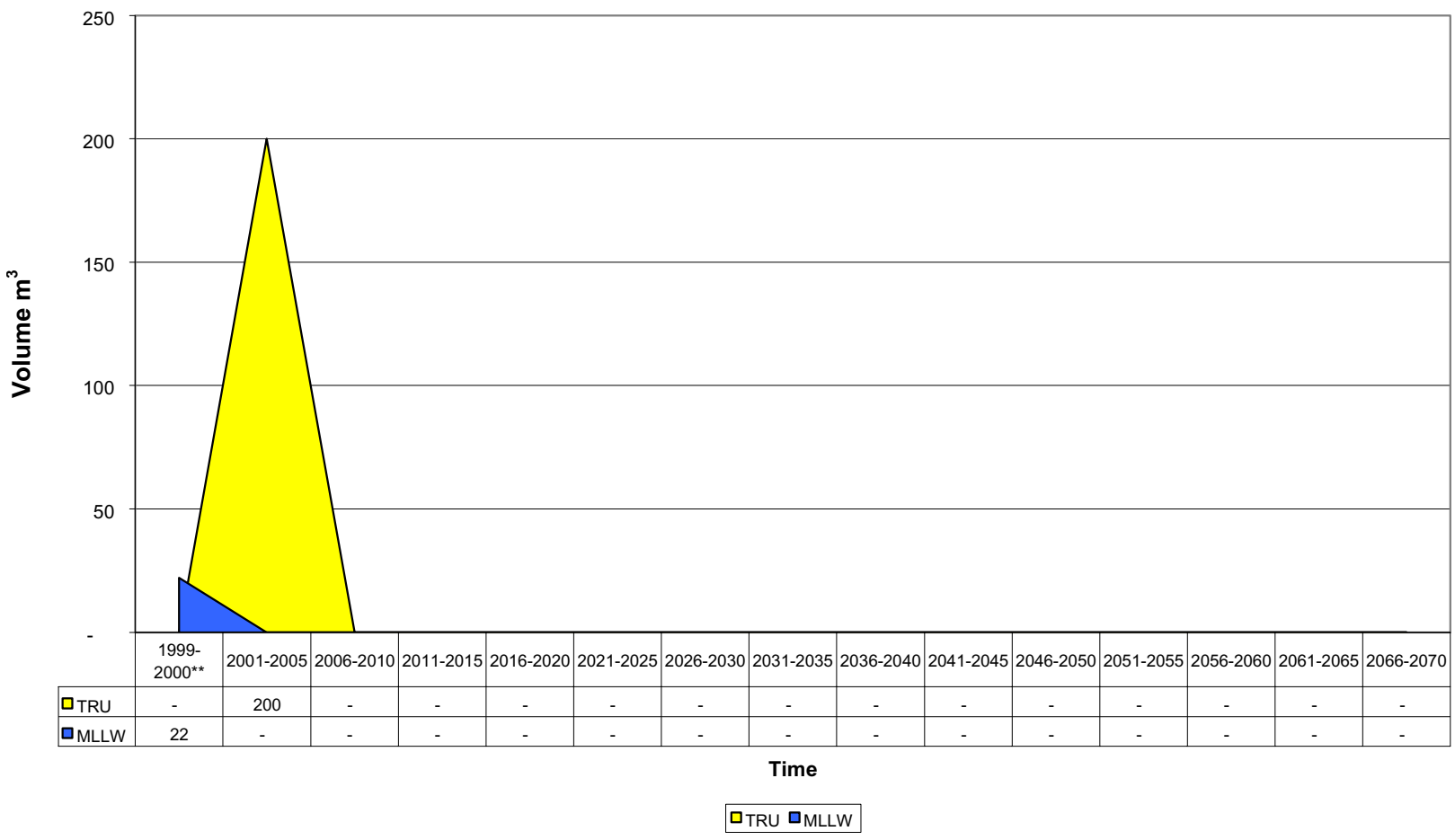

Rocky Flats

Outgoing LLW Volume to Nevada Test Site

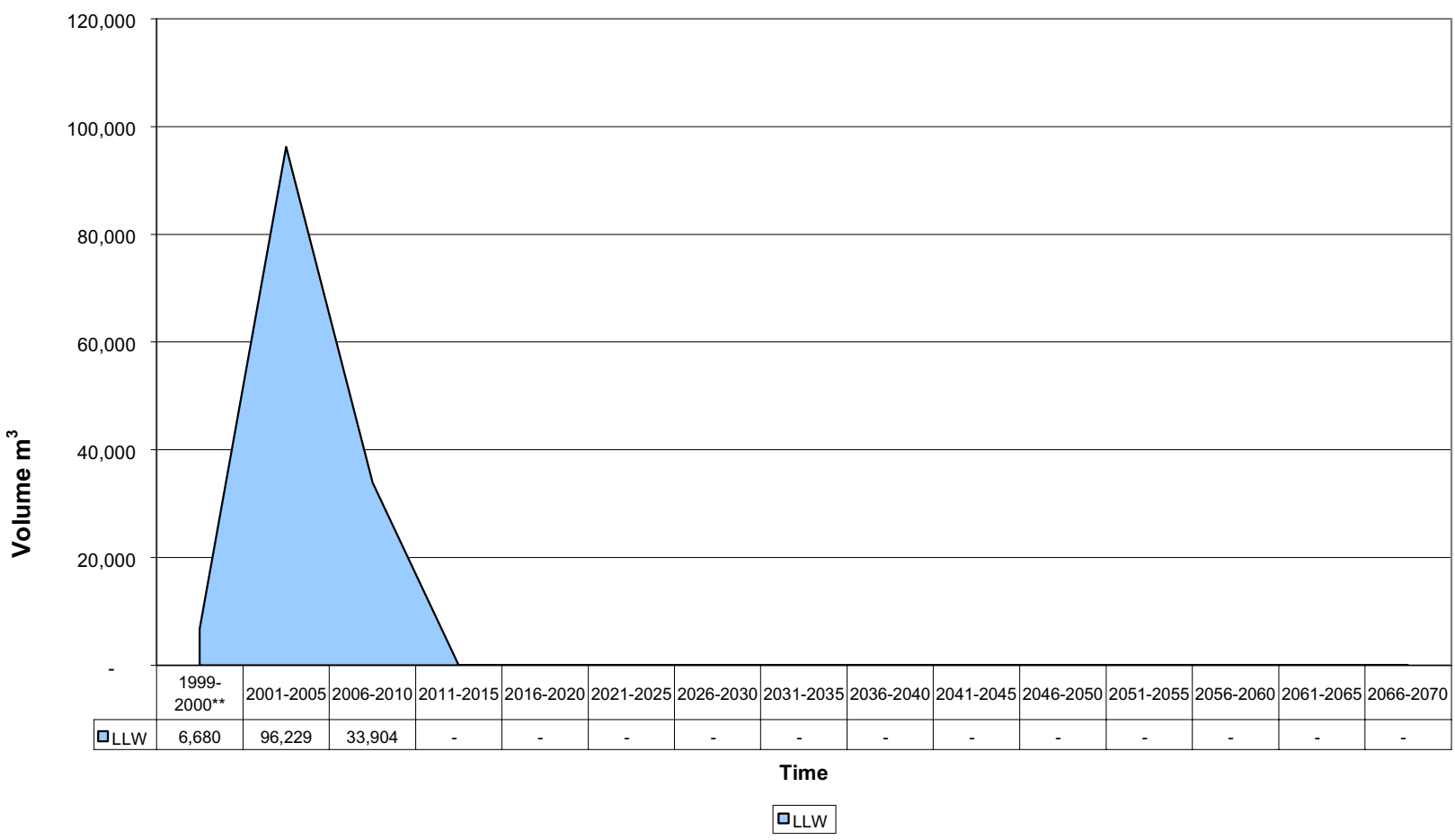


Rocky Flats

Outgoing M/LLW Volume to Oak Ridge

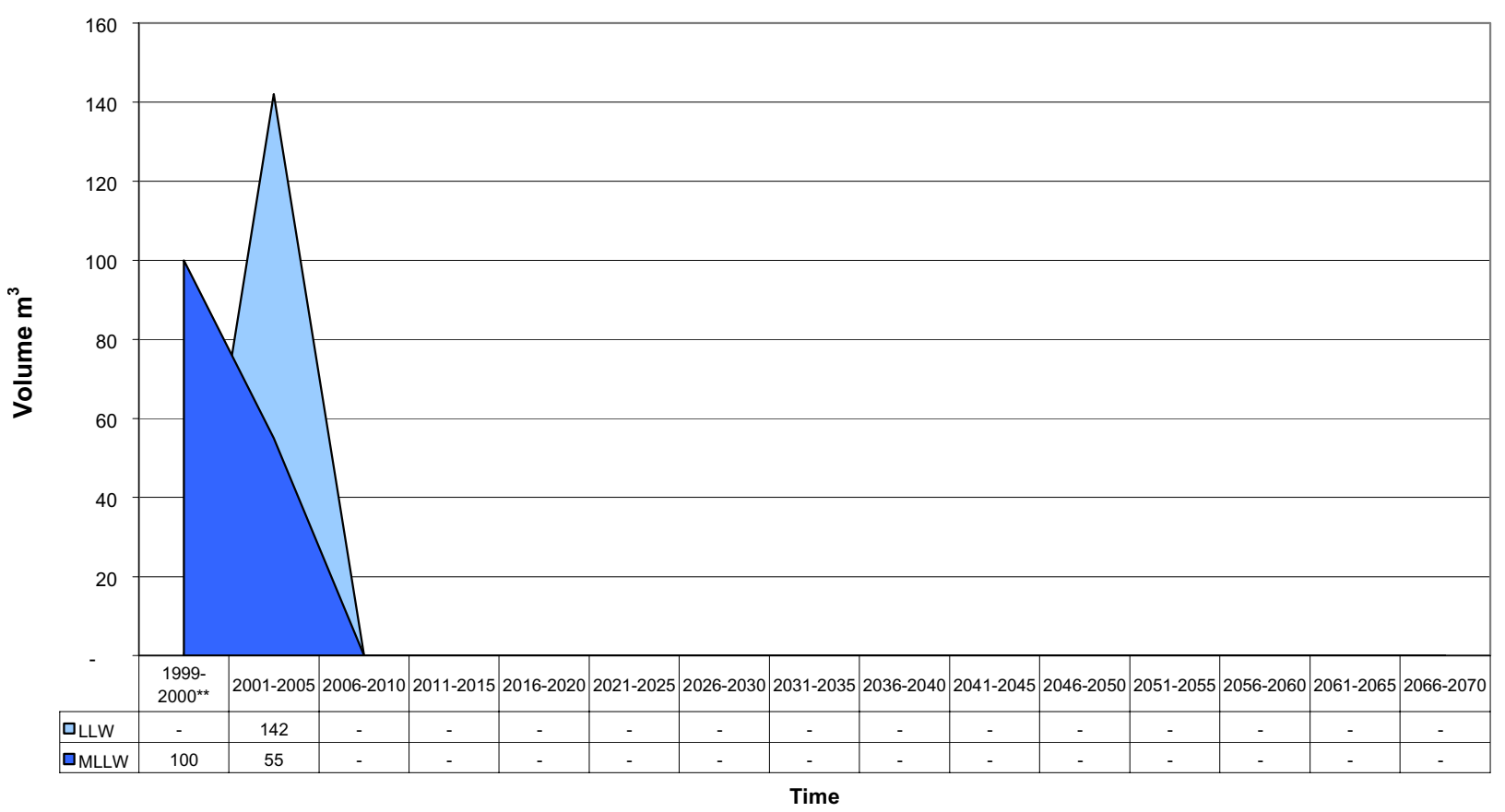

口LLW םMLLW

Rocky Flats

Outgoing MLLW Volume to "TBDO"

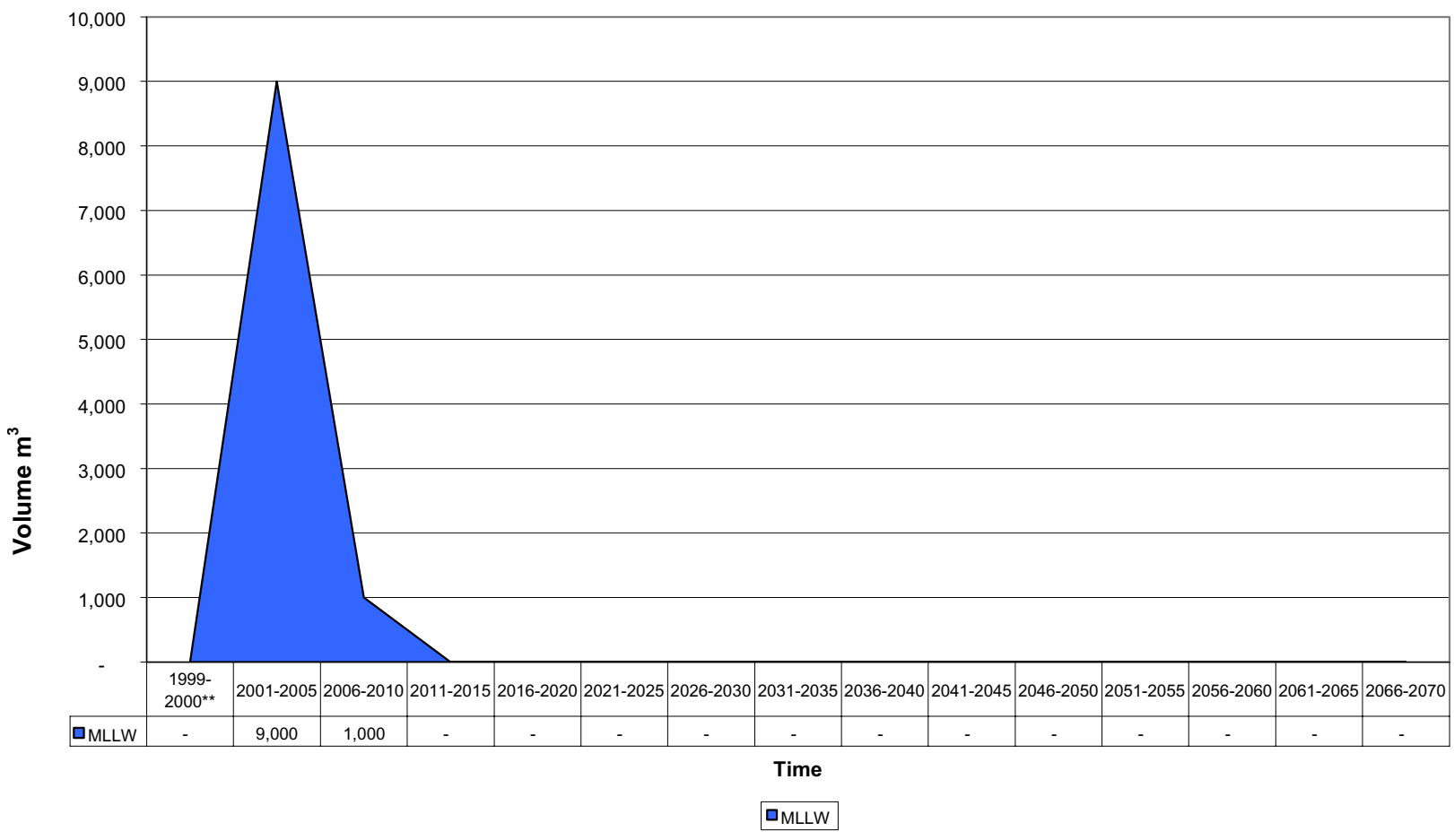


Rocky Flats

Outgoing TRU Volume to WIPP

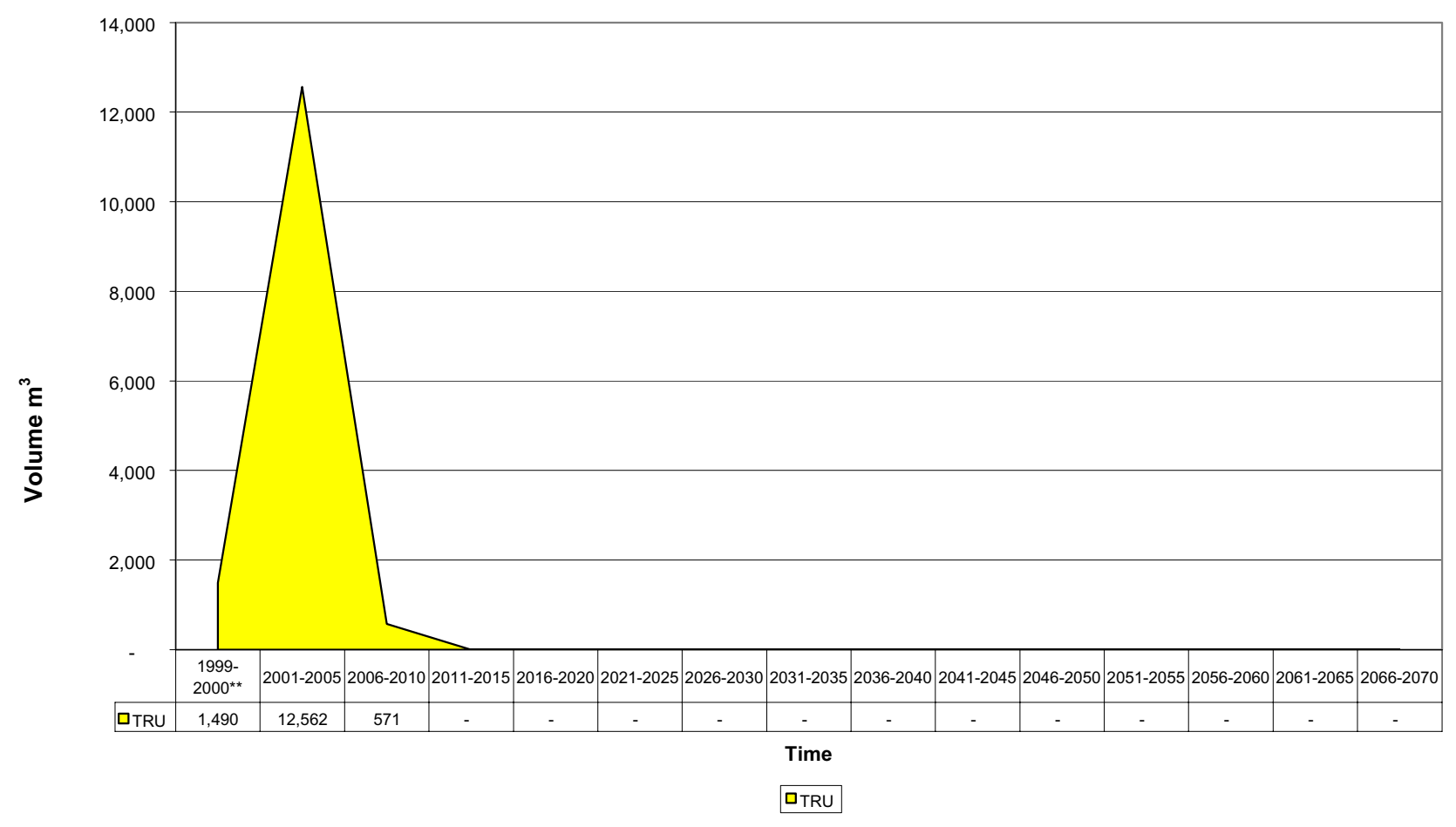




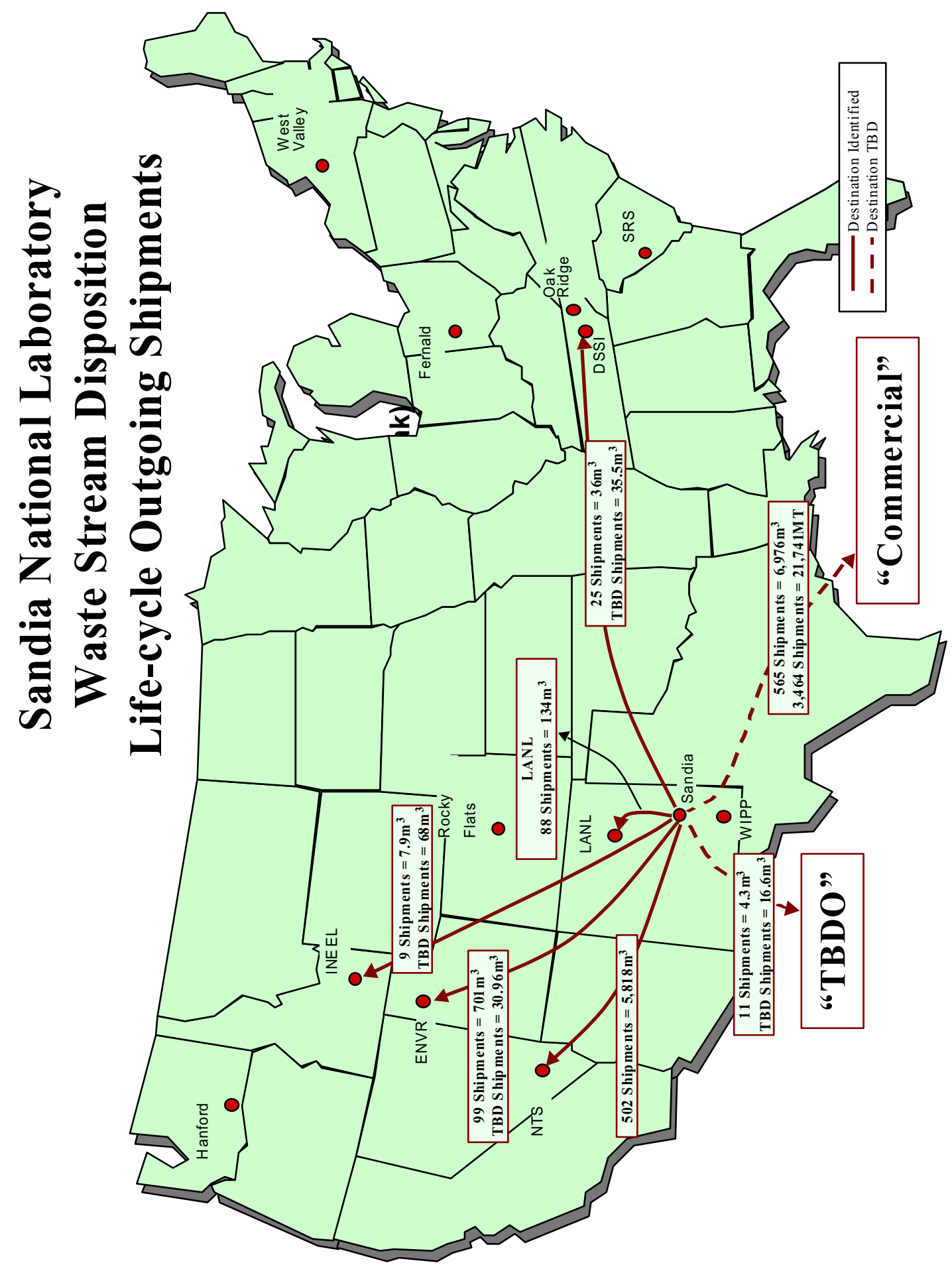


Sanida National Laboratory

Waste Stream Disposition

Life-cycle Outgoing Shipments

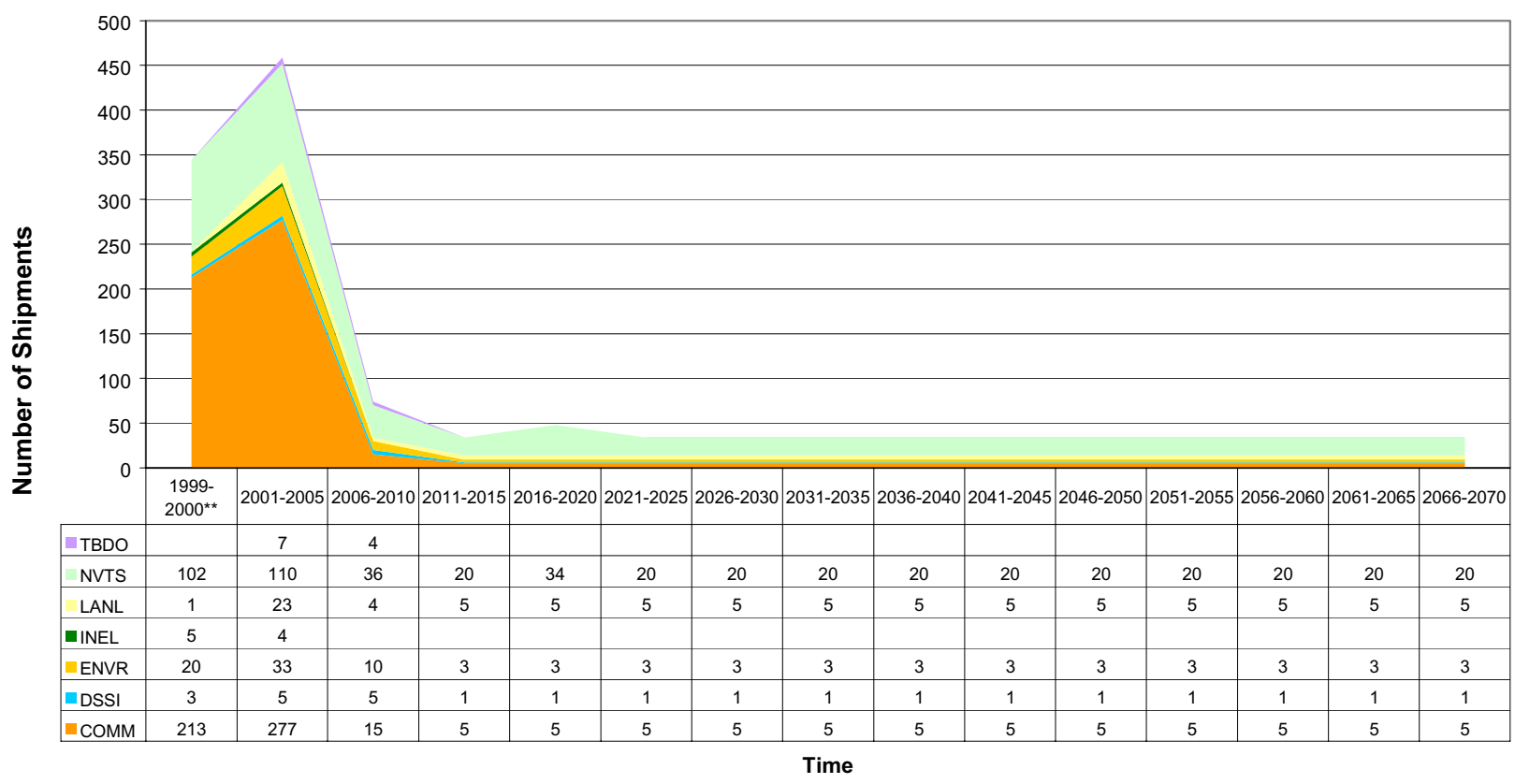

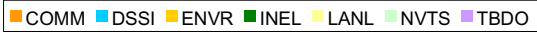

Sandia National Laboratory

Outgoing MLLW Shipments to INEEL

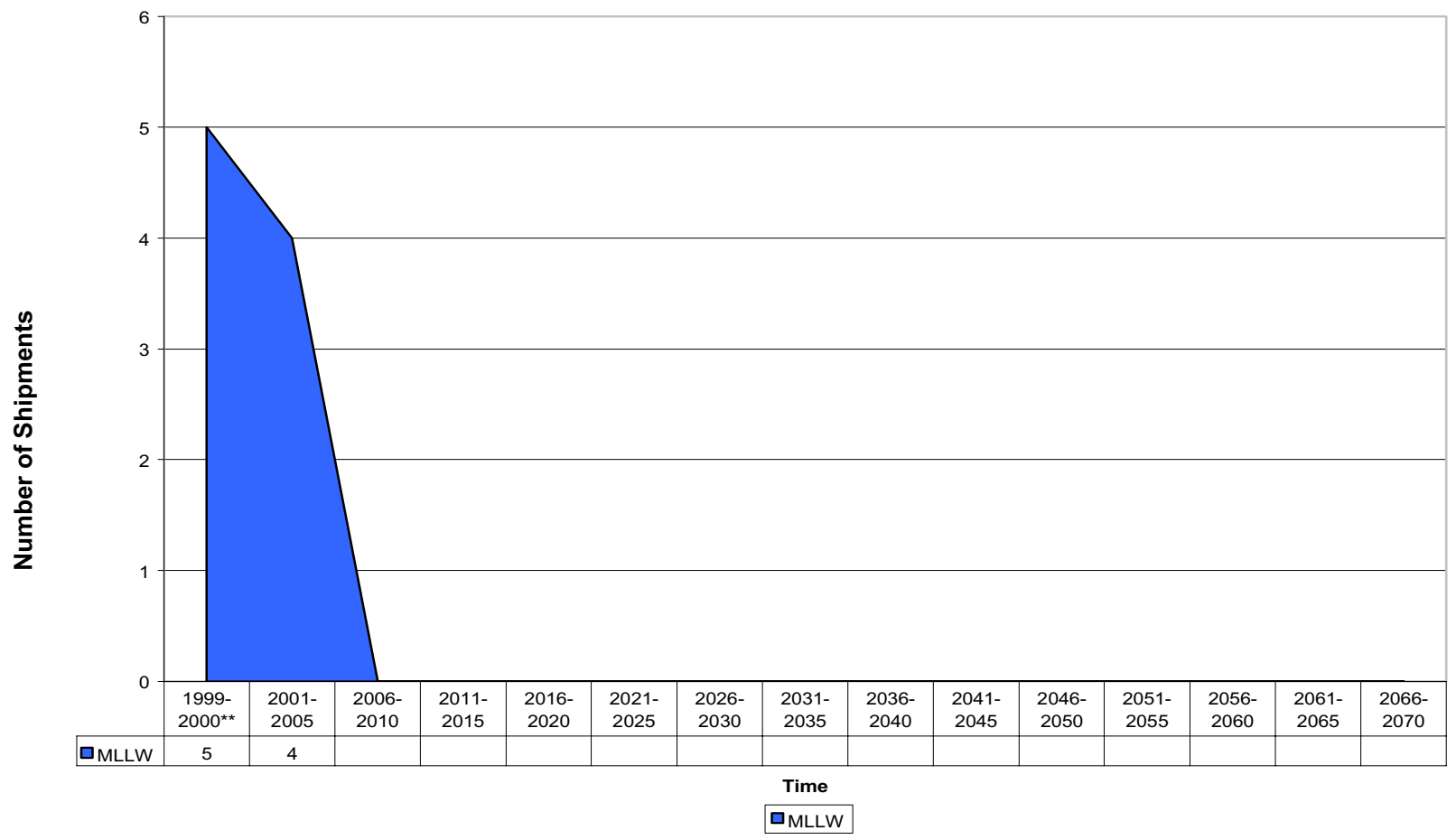

Total life-cycle values per 7/30/99 Stream Disposition Data (SDD) (http://id.inel.gov/avs/).

** Represents data for a two year period. 


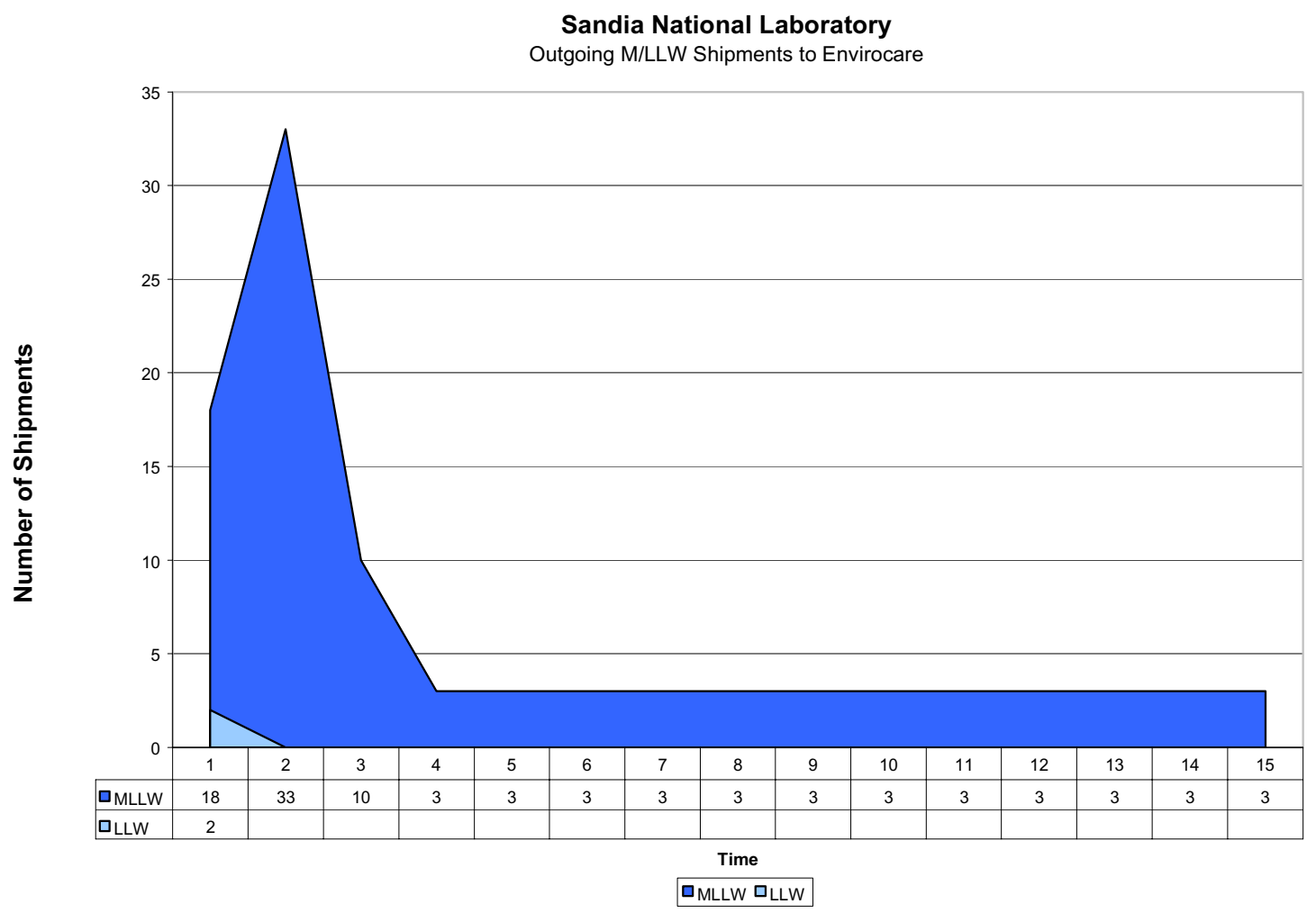

Sandia National Laboratory

Outgoing LLW Shipments to Nevada Test Site

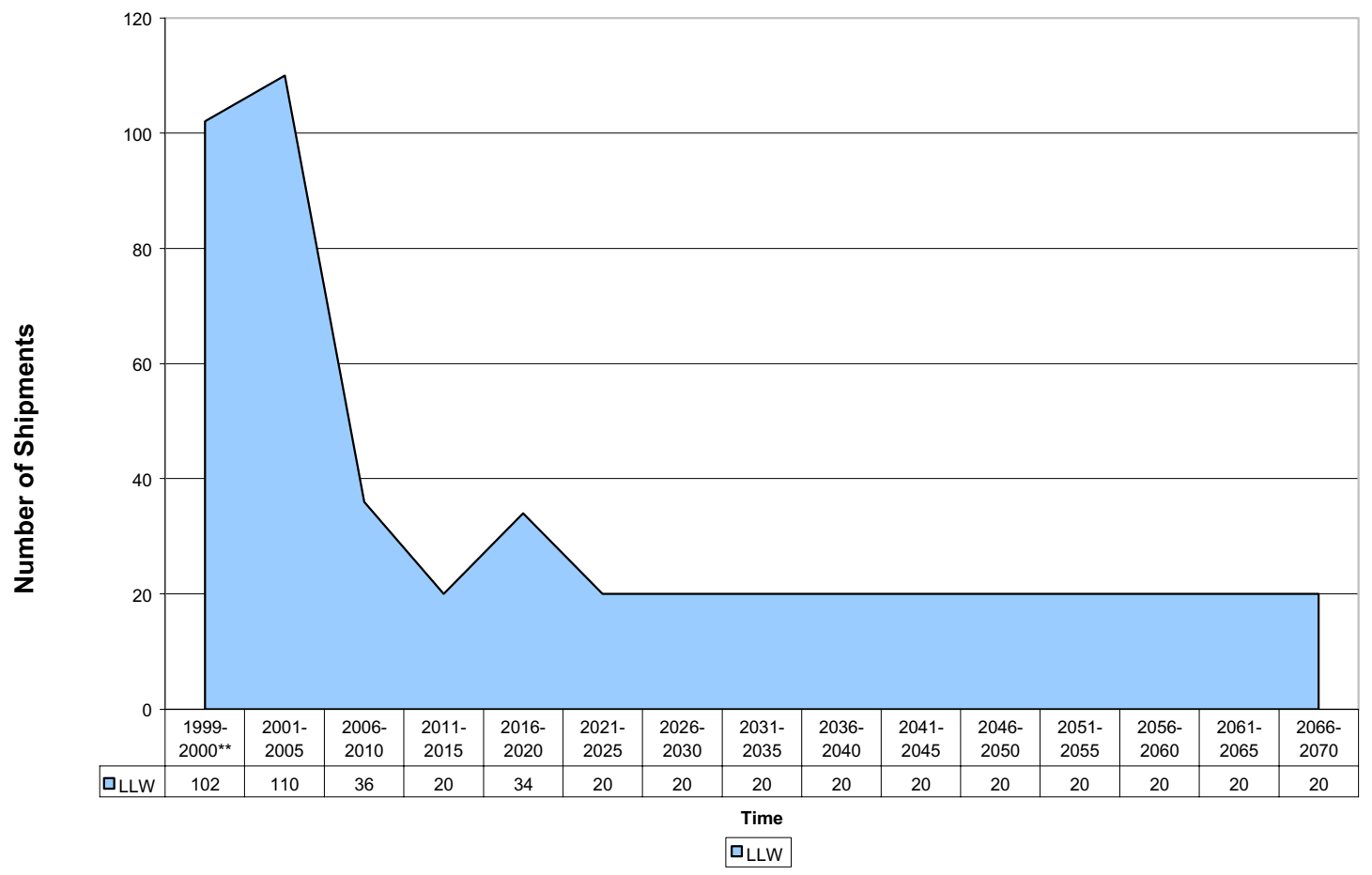

Total life-cycle values per 7/30/99 Stream Disposition Data (SDD) (http://id.inel.gov/avs/).

** Represents data for a two year period. 


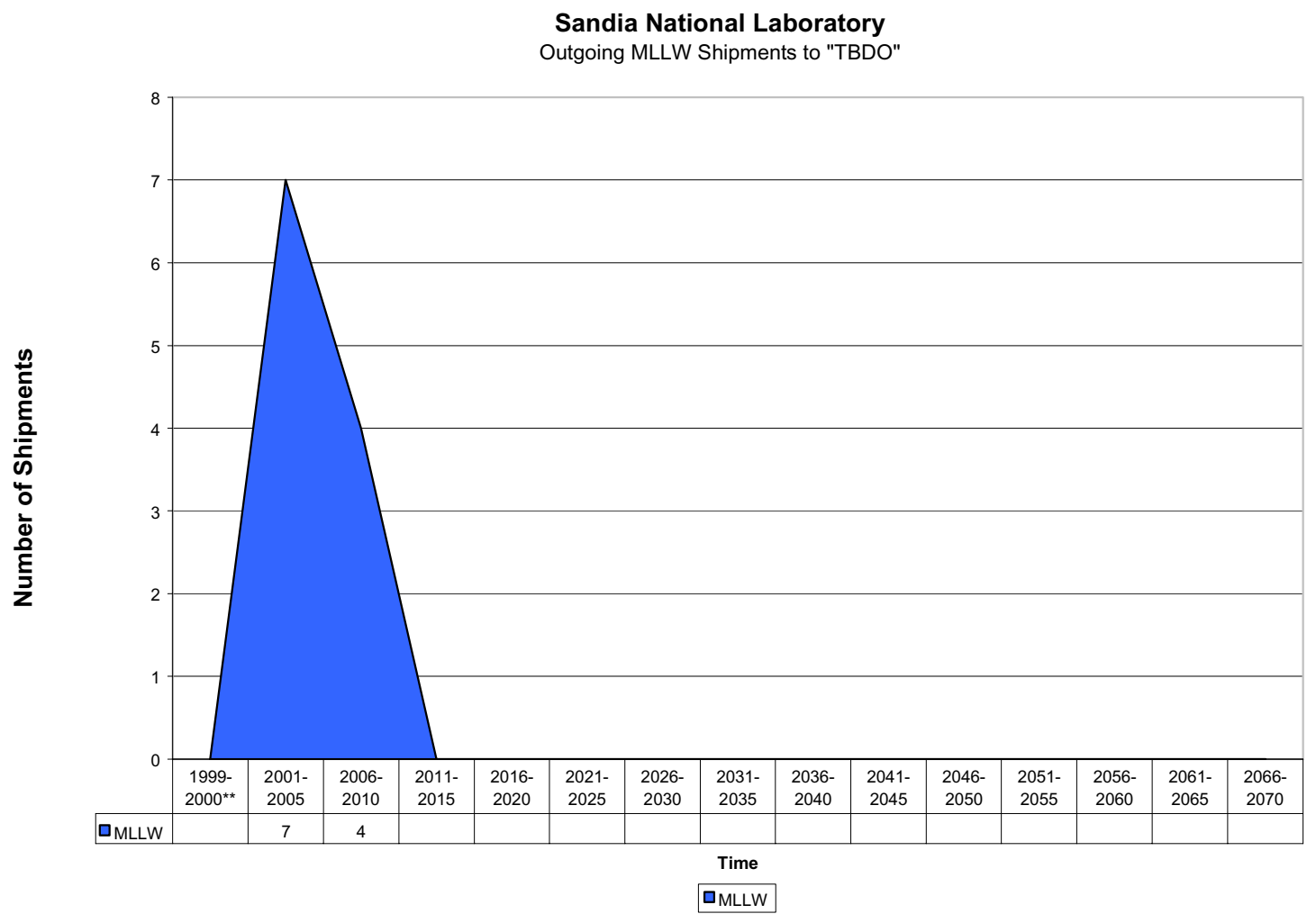

Sandia National Laboratory

Outgoing TRU Shipments to Los Alamos National Laboratory

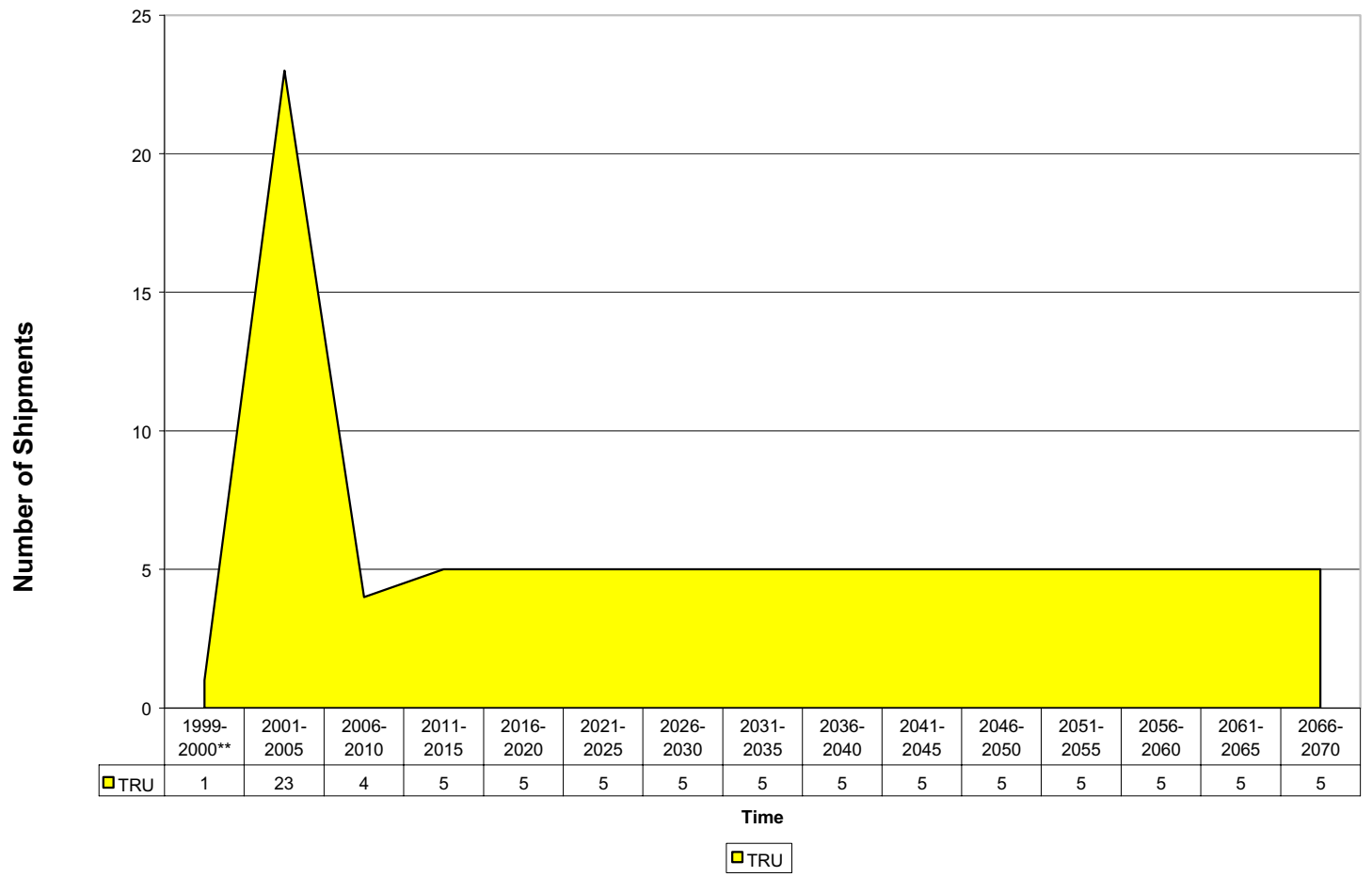

Total life-cycle values per 7/30/99 Stream Disposition Data (SDD) (http://id.inel.gov/avs/).

** Represents data for a two year period. 
Sandia National Laboratory

Outgoing MLLW Shipments to Diversified Scientific Services, Inc.

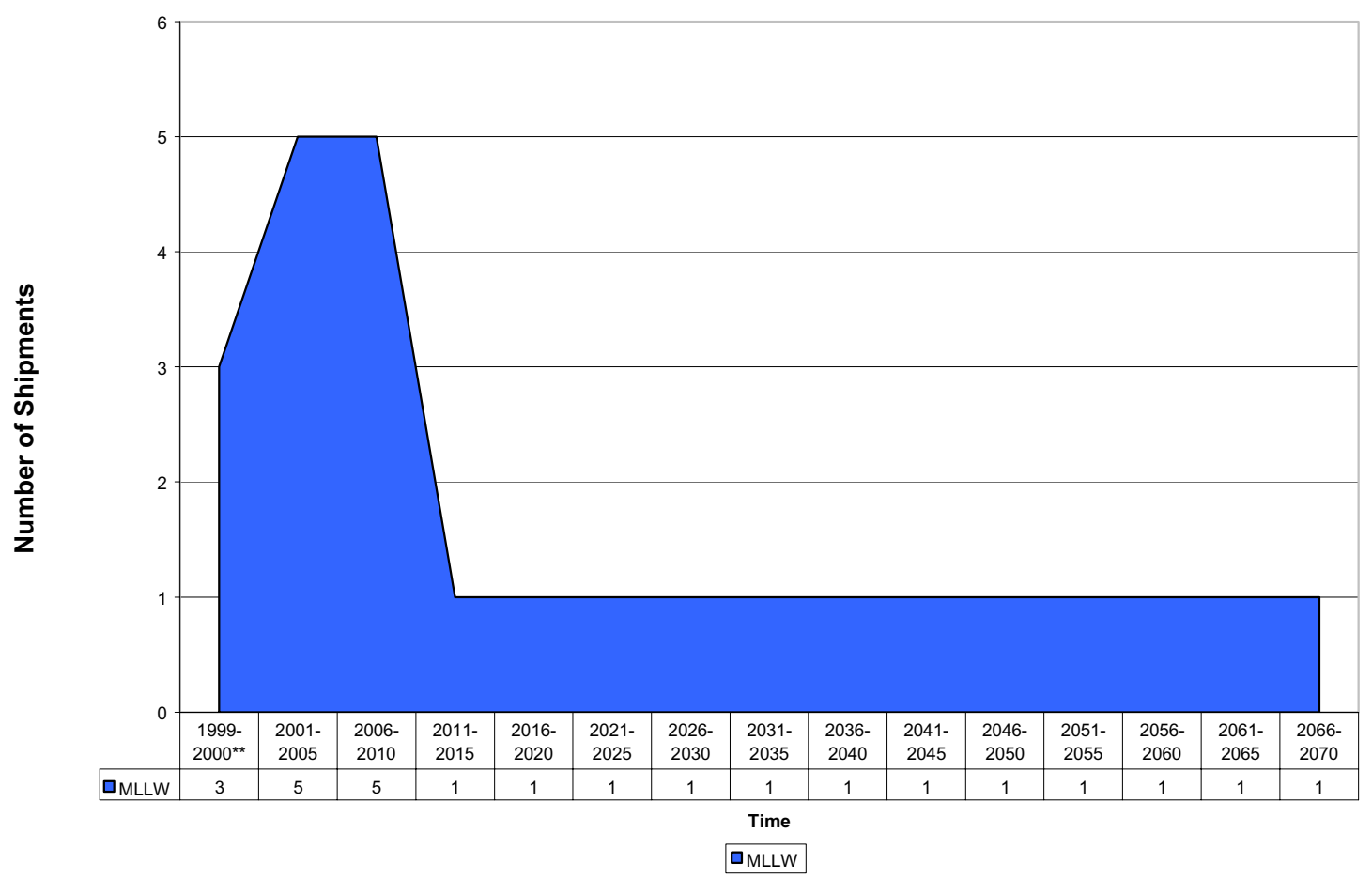

Sandia National Laboratory

Outgoing M/LLW Shipments to "Commercial"

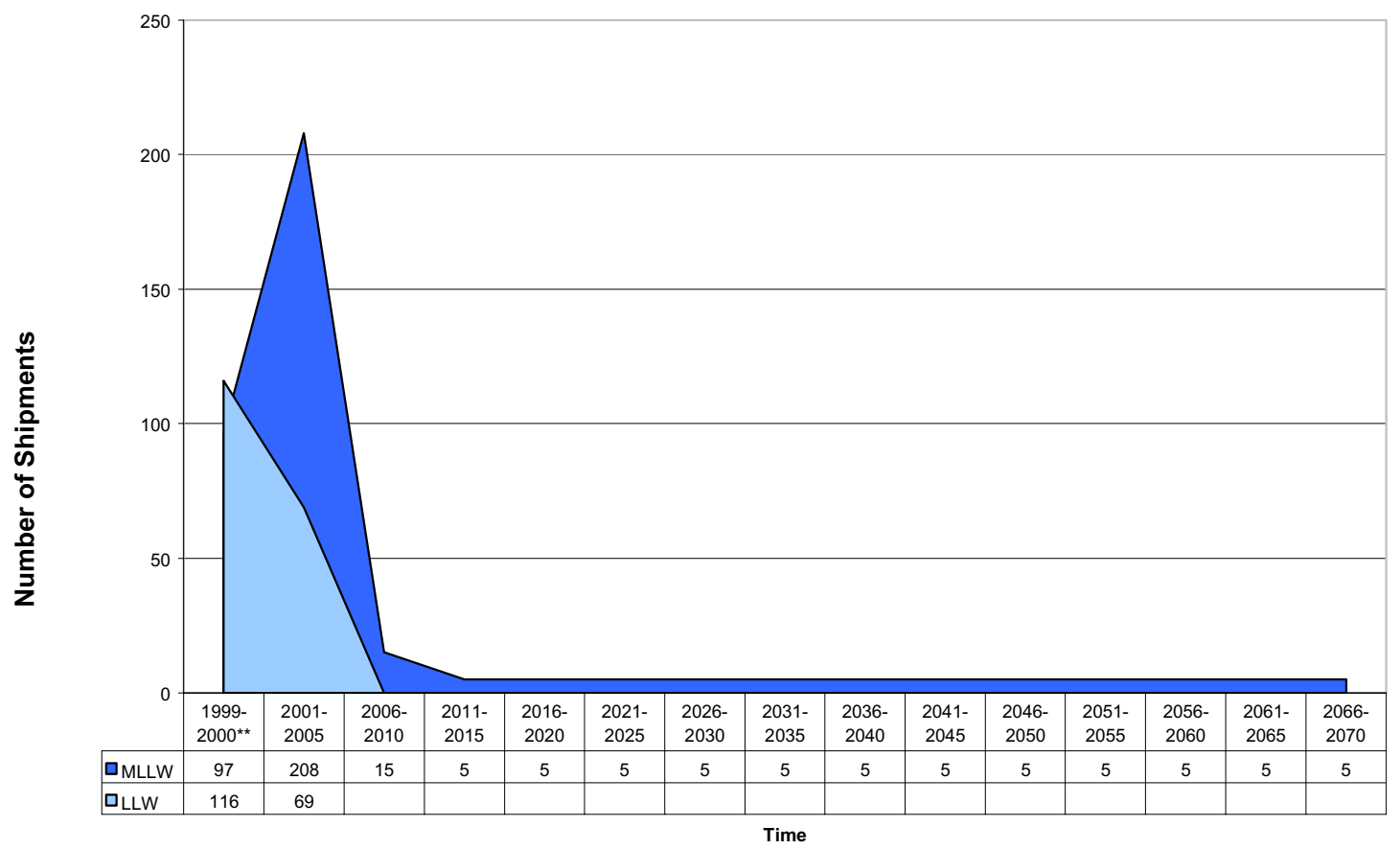

口MLLW DLLW

Total life-cycle values per 7/30/99 Stream Disposition Data (SDD) (http://id.inel.gov/avs/).

** Represents data for a two year period. 


\section{Sandia National Laboratory \\ Waste Stream Disposition \\ Life-cycle Outgoing Volume}

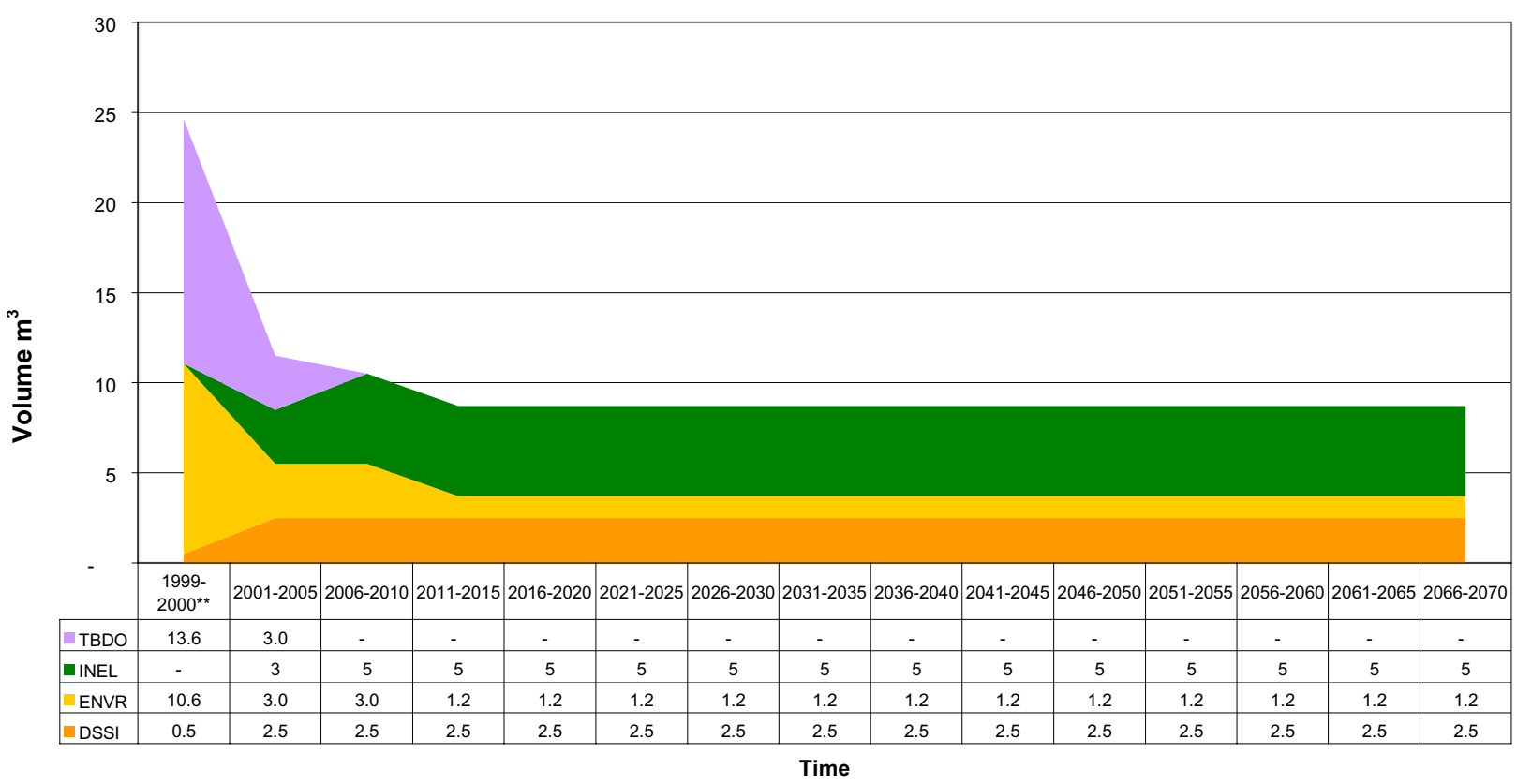

$\square$ DSSI $₫$ ENVR $\square$ INEL $\amalg$ TBDO

Sandia National Laboratory

Outgoing MLLW Volume to Diversified Scientific Services, Inc.

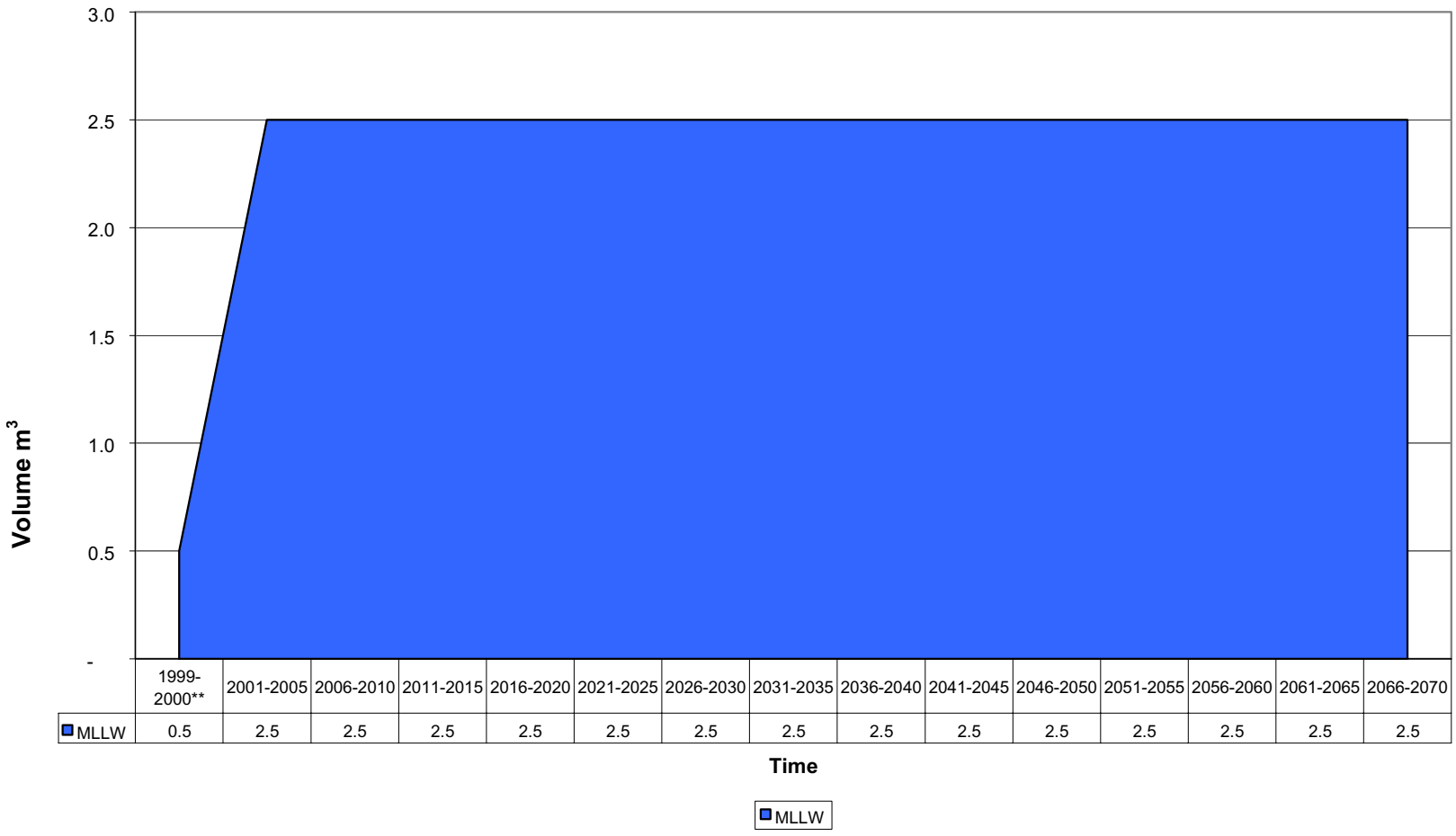

Total life-cycle values per 7/30/99 Stream Disposition Data (SDD) (http://id.inel.gov/avs/).

** Represents data for a two year period. 
Sandia National Laboratory

Outgoing MLLW Volume to Envirocare

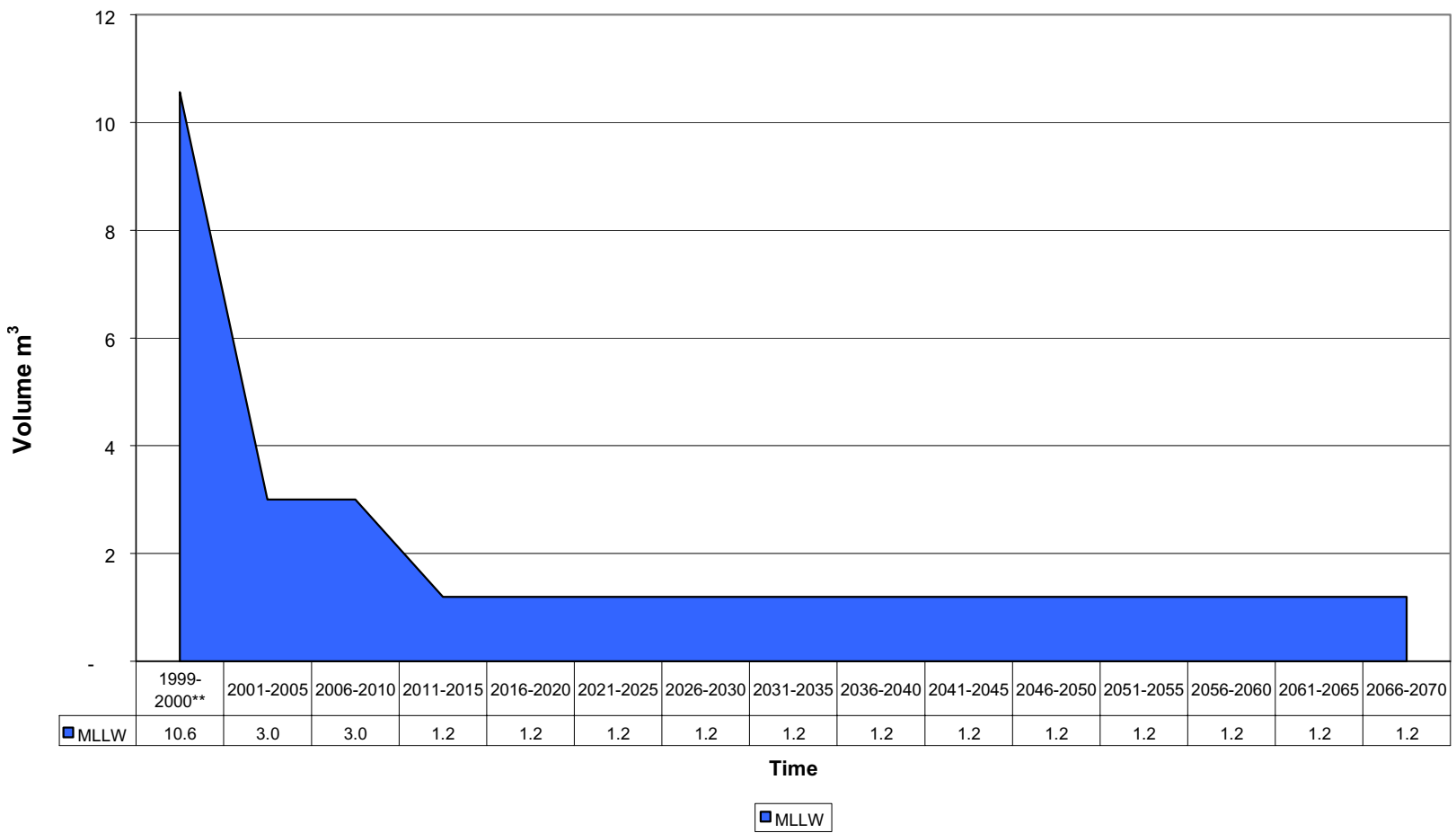

Sandia National Laboratory

Outgoing MLLW Volume to INEEL

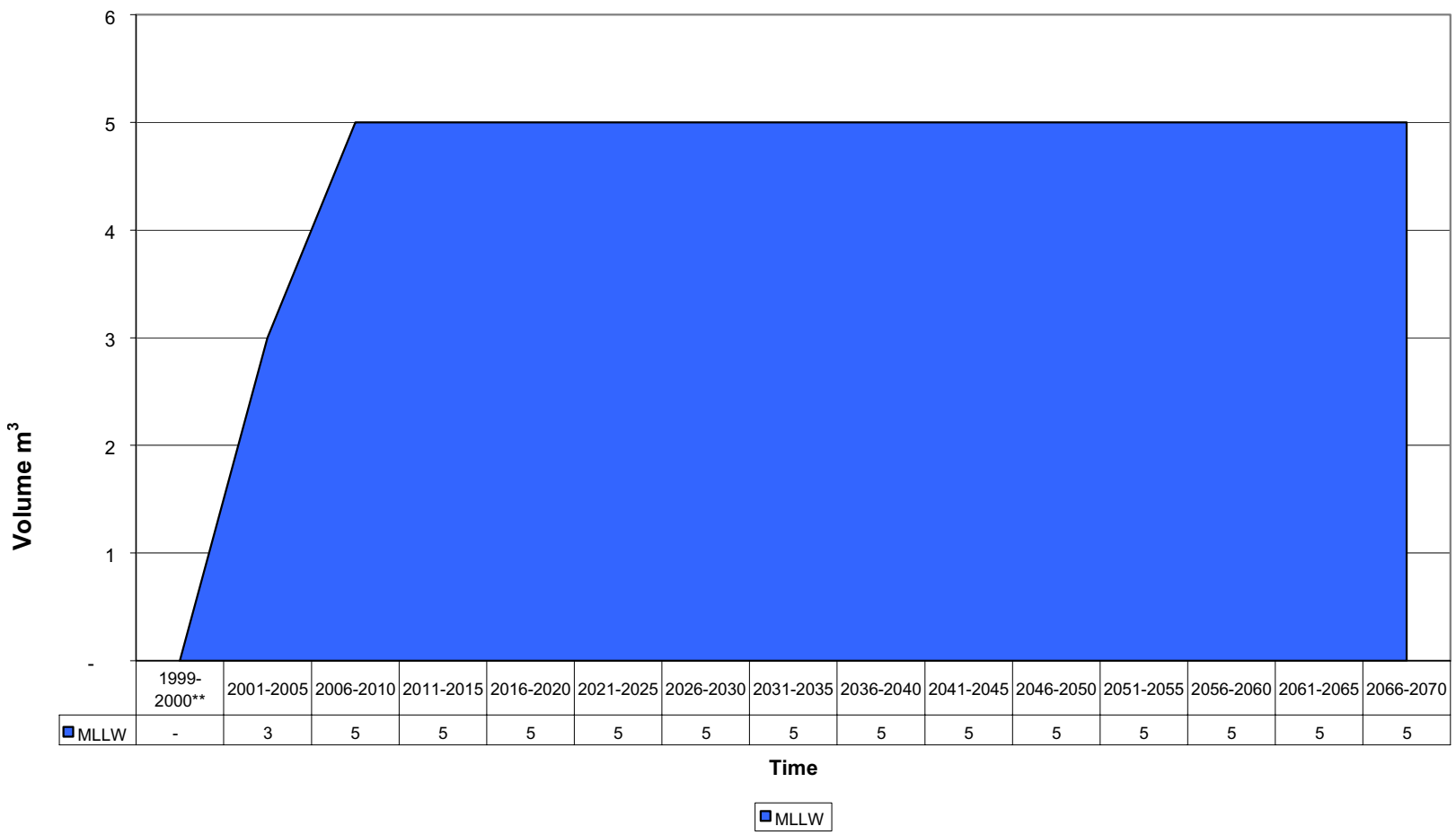

Total life-cycle values per 7/30/99 Stream Disposition Data (SDD) (http://id.inel.gov/avs/).

** Represents data for a two year period. 


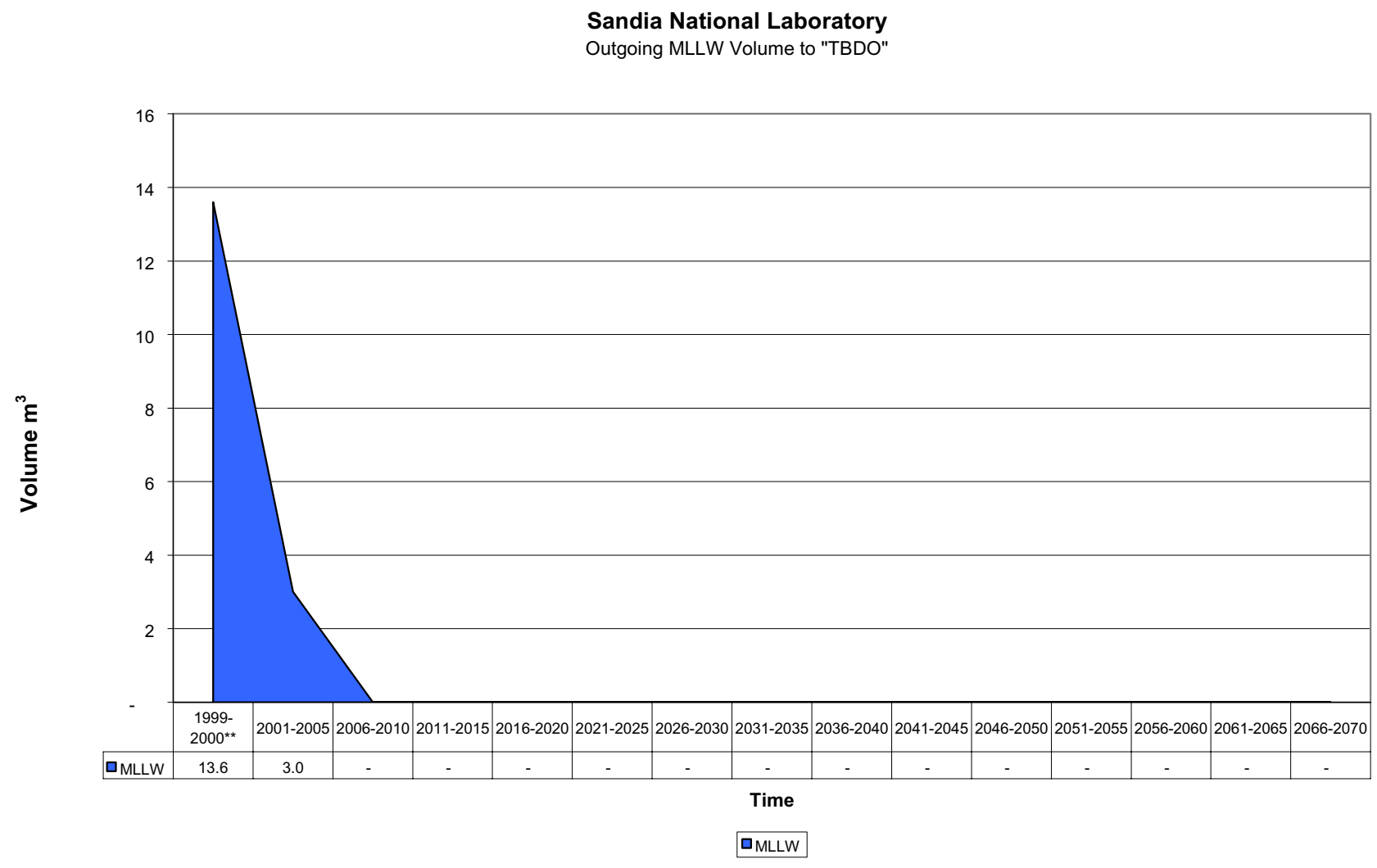

Total life-cycle values per 7/30/99 Stream Disposition Data (SDD) (http://id.inel.gov/avs/).

** Represents data for a two year period. 


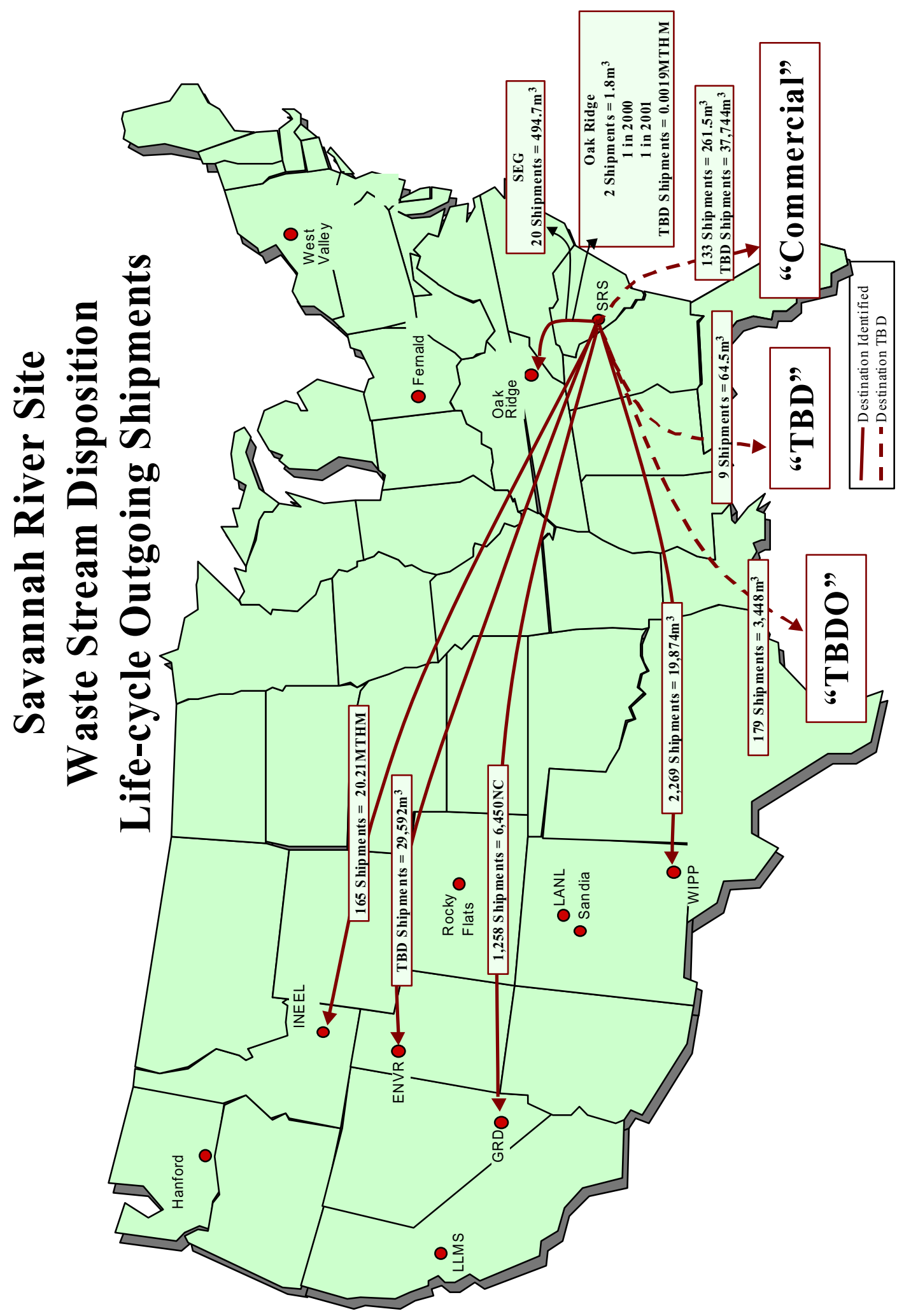


Savannah River Site

Waste Stream Disposition

Life-cycle Outgoing Shipments

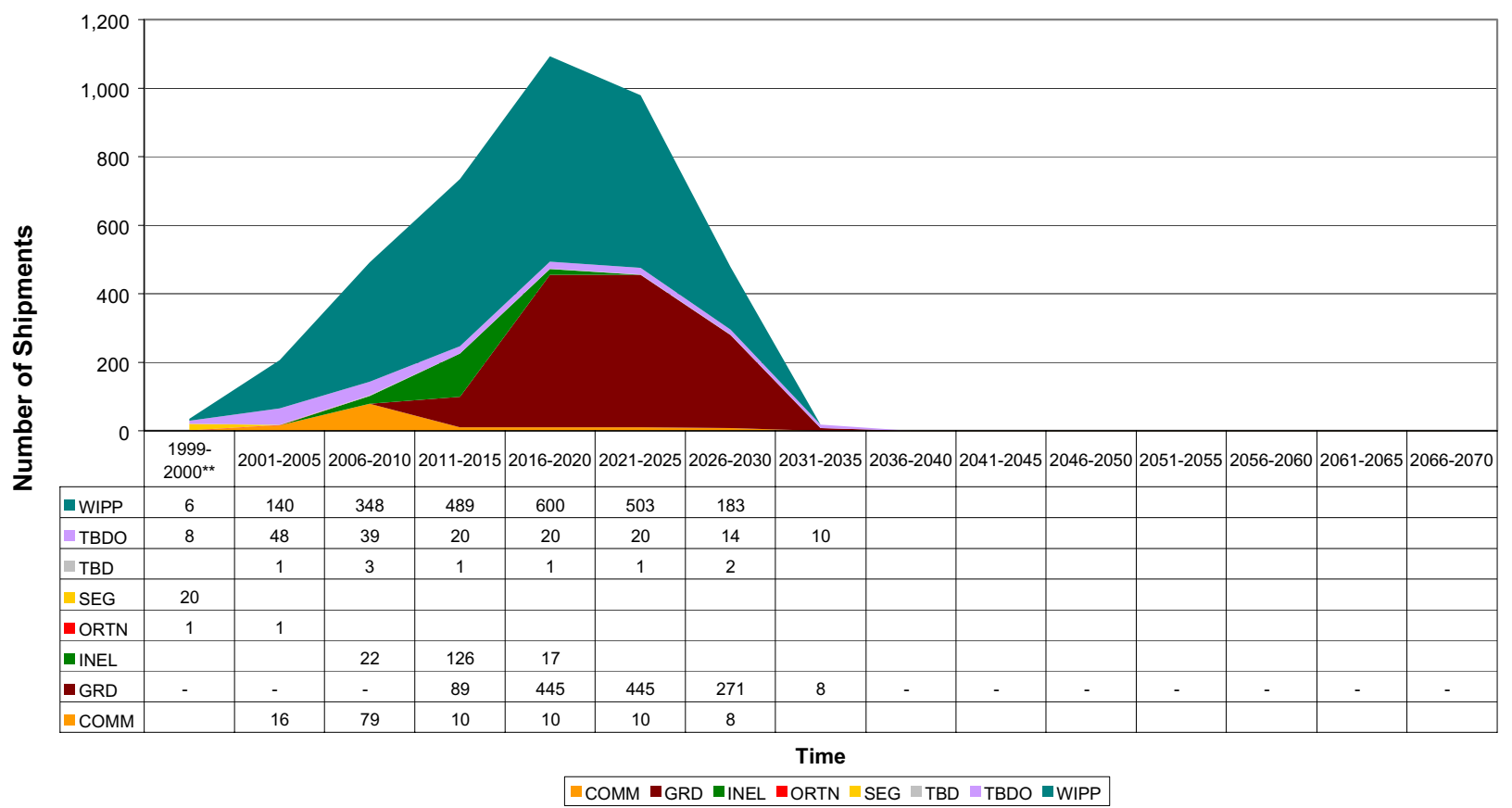

Savannah River Site

Outgoing HLW \& SNF Shipments to Geologic Repository Disposal

(Tentatively Yucca Mtn.)

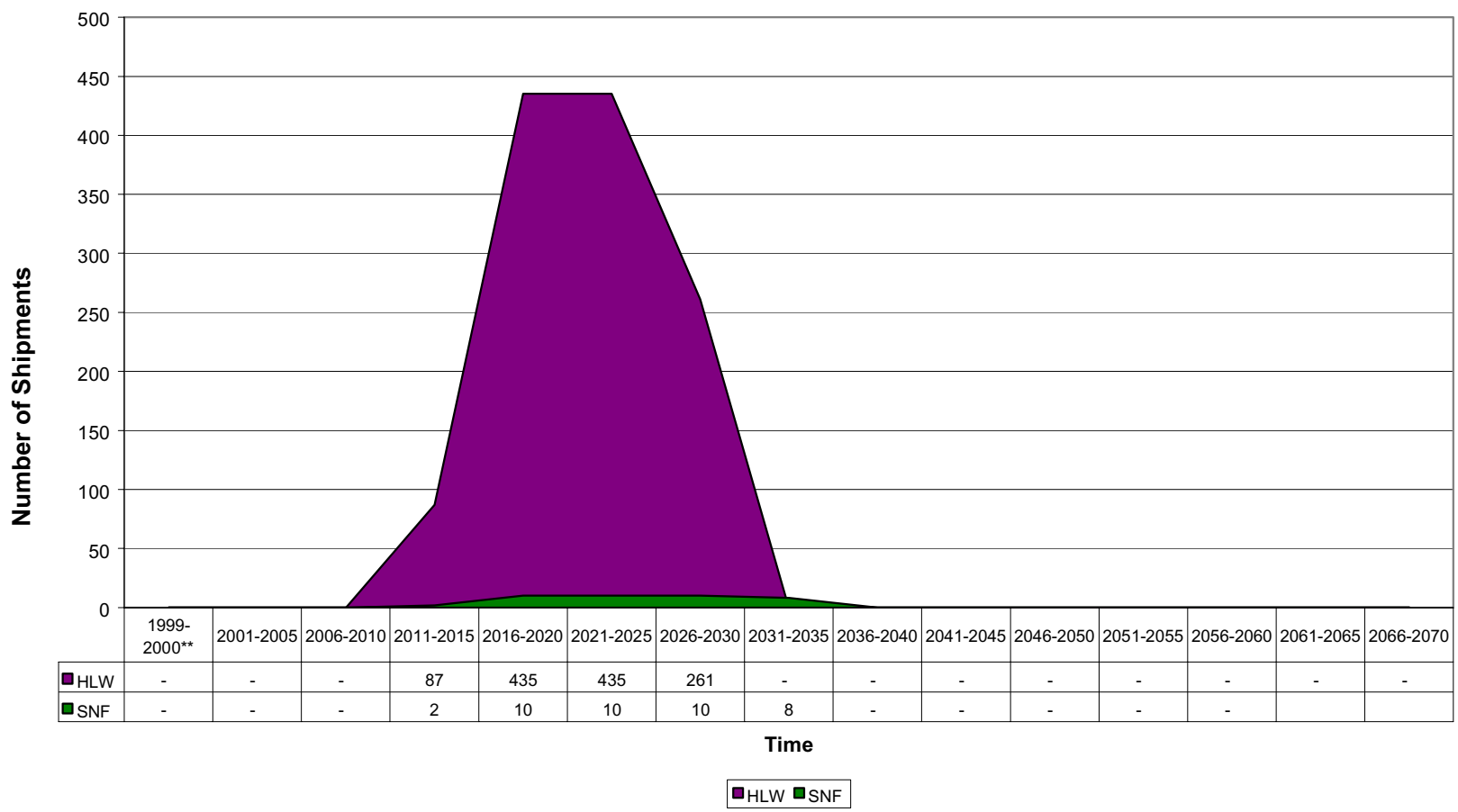

Total life-cycle values per 7/30/99 Stream Disposition Data (SDD) (http://id.inel.gov/avs/) 
Savannah River Site

Outgoing SNF Shipments to INEEL

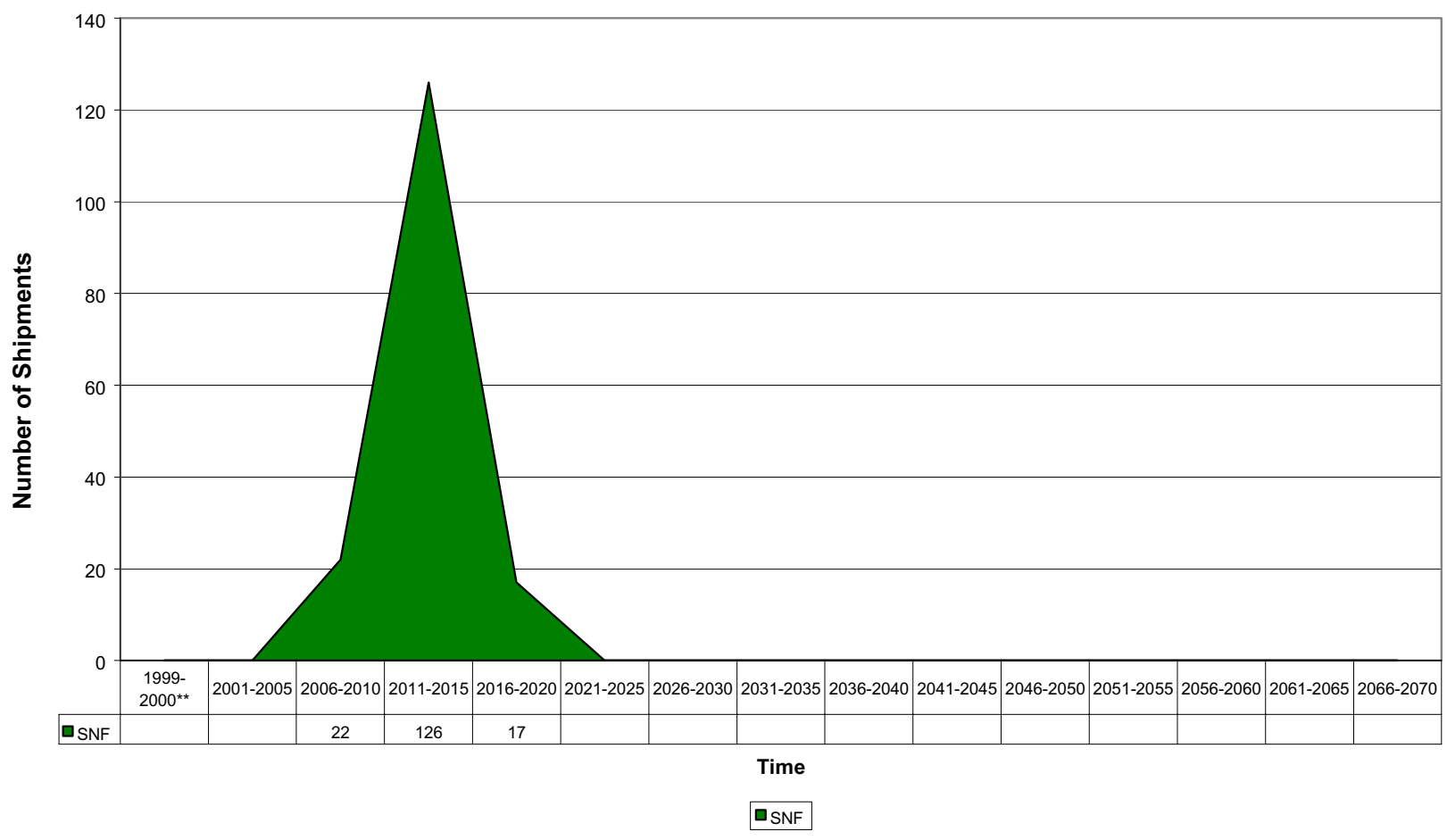

Savannah River Site

Outgoing MLLW Shipments to "Commercial"

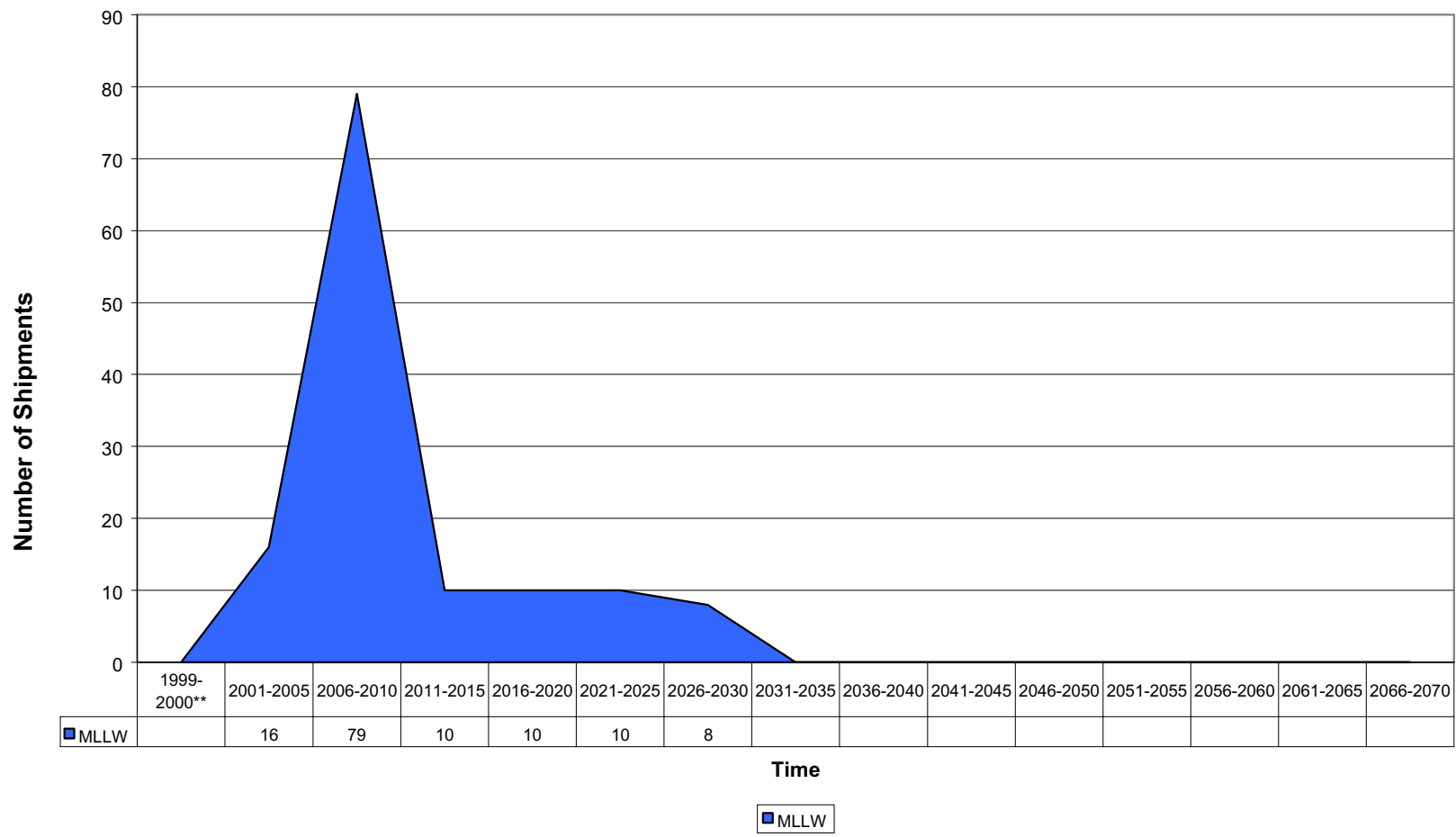

Total life-cycle values per 7/30/99 Stream Disposition Data (SDD) (http://id.inel.gov/avs/) 
Savannah River Site

Outgoing MLLW Shipments to "TBDO"

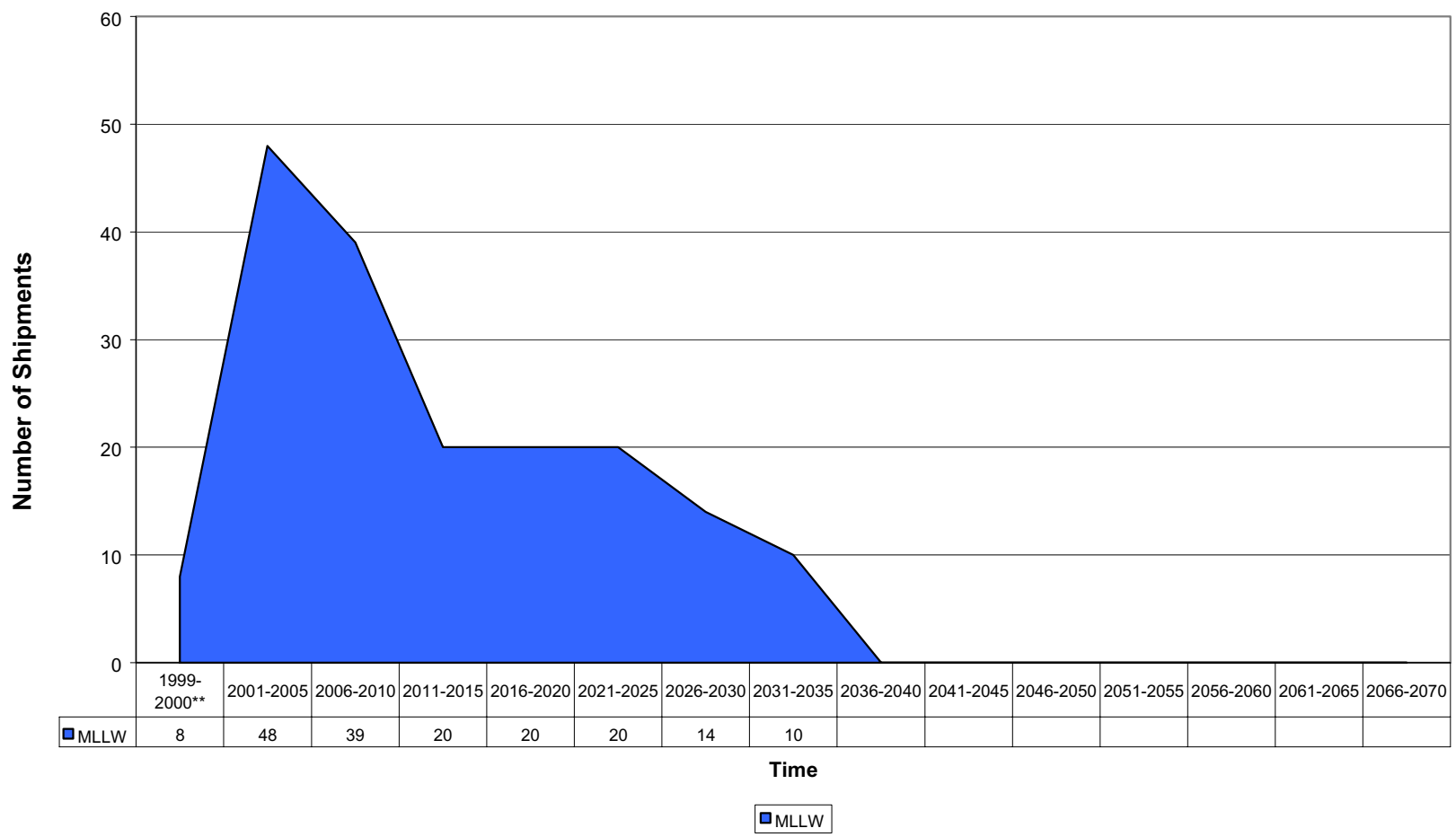

Savannah River Site

Outgoing TRU Shipments to WIPP

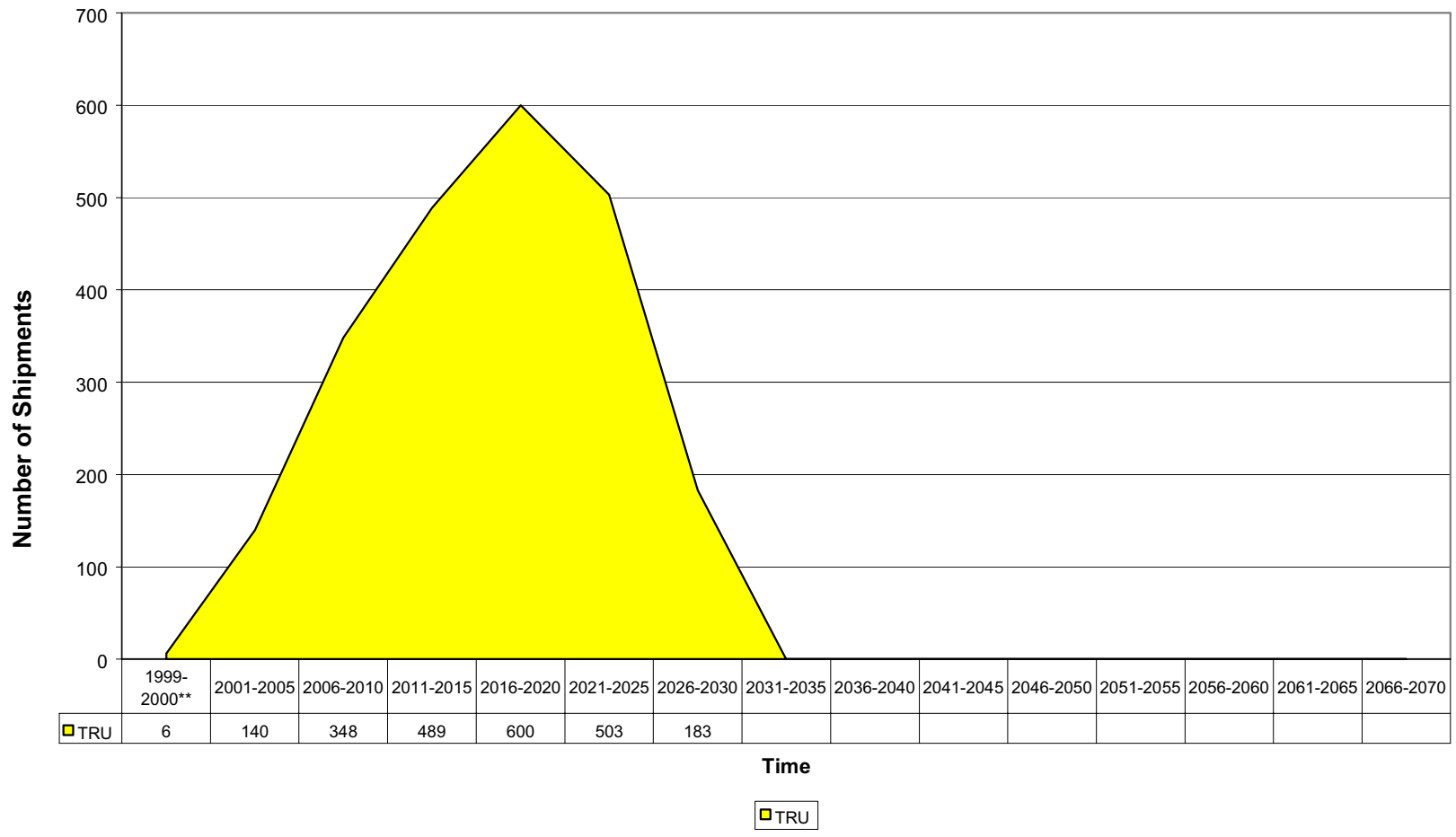

Total life-cycle values per 7/30/99 Stream Disposition Data (SDD) (http://id.inel.gov/avs/) 
Savannah River Site

Outgoing MLLW Shipments to "TBD"

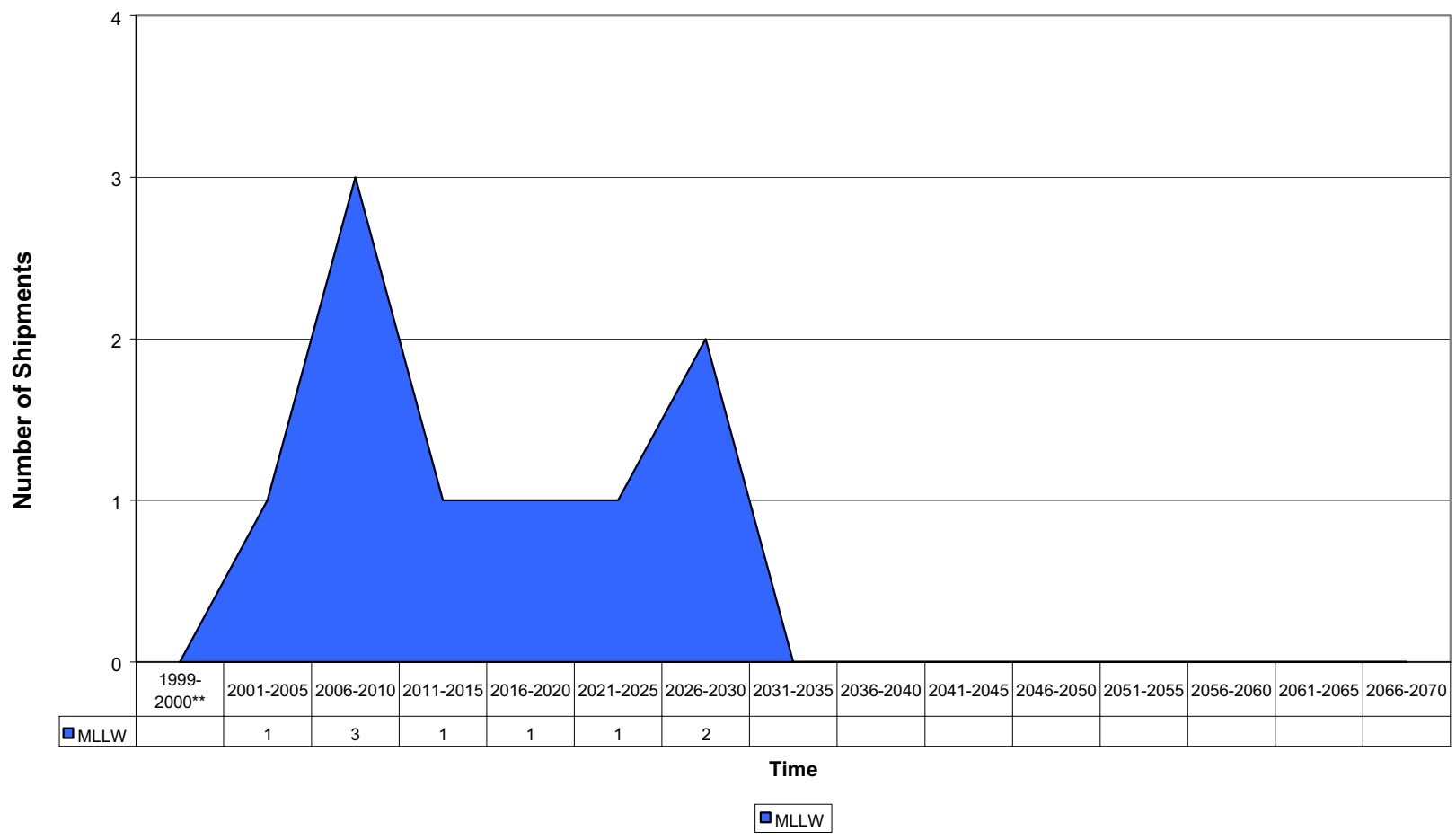

Savannah River Site

Outgoing LLW Shipments to GTS Duratek (SEG)

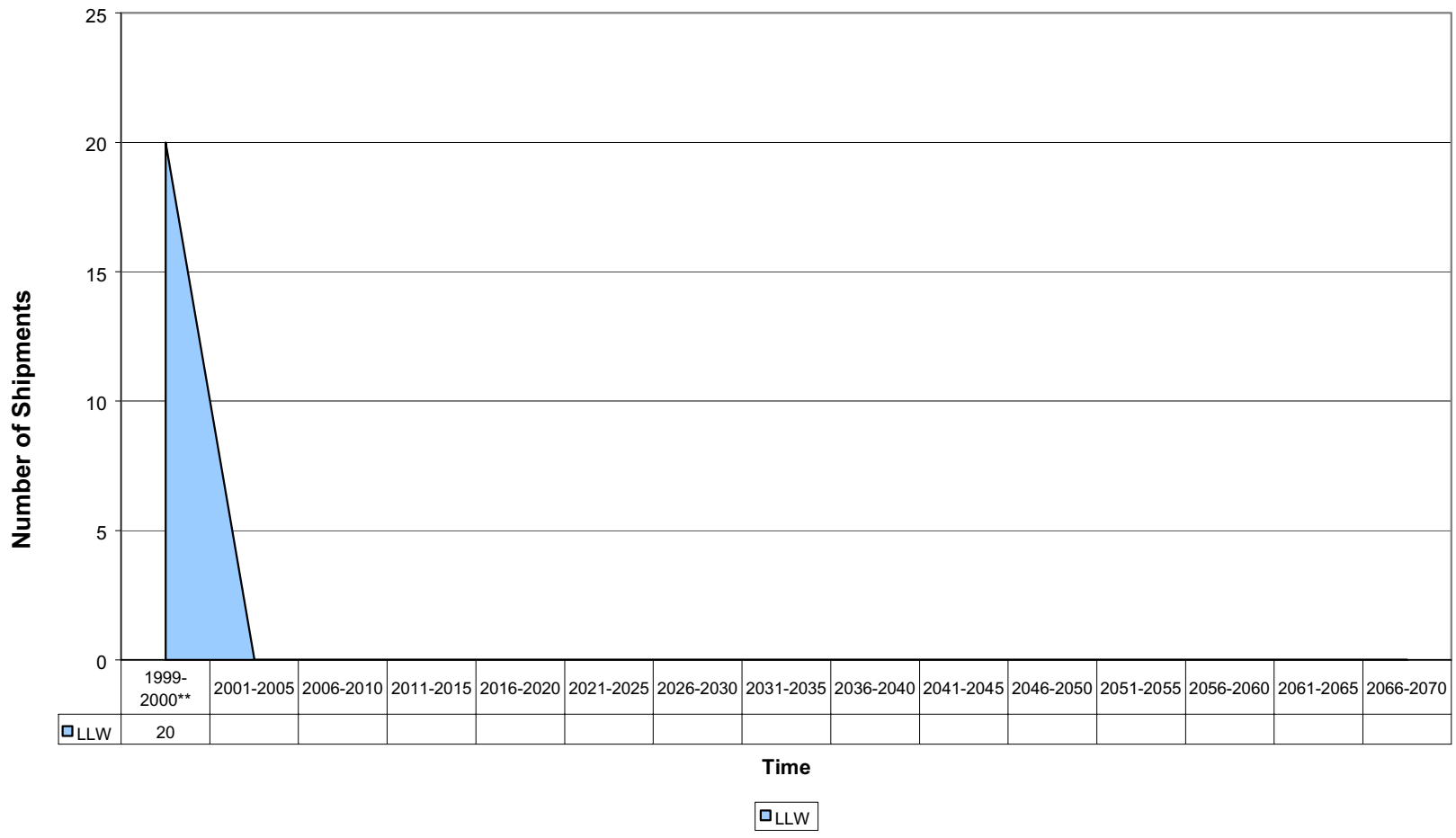

Total life-cycle values per 7/30/99 Stream Disposition Data (SDD) (http://id.inel.gov/avs/) 
Savannah River Site

Waste Stream Disposition

Life-cycle Outgoing Volume

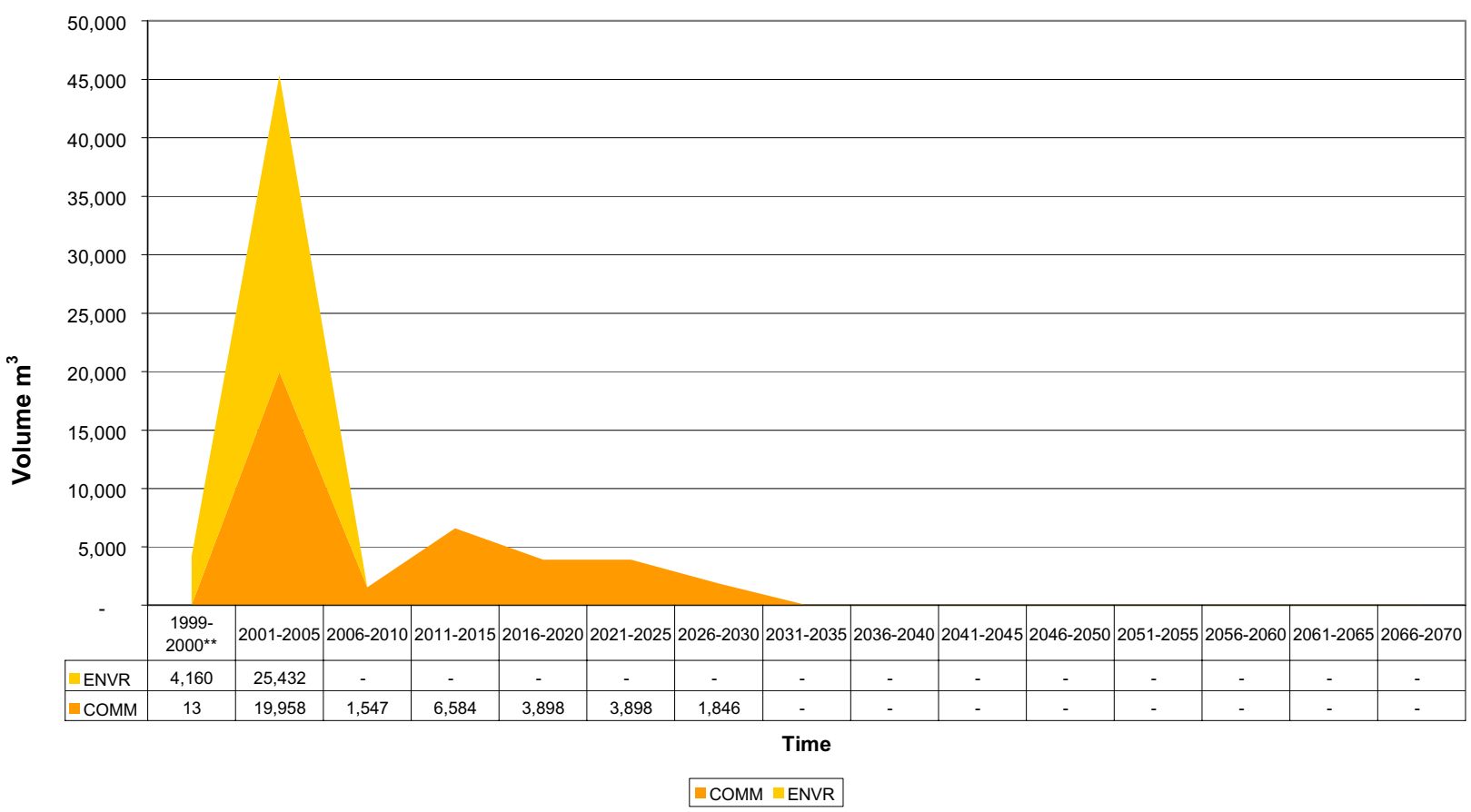

Savannah River Site

Outgoing LLW Volume to "Commercial"

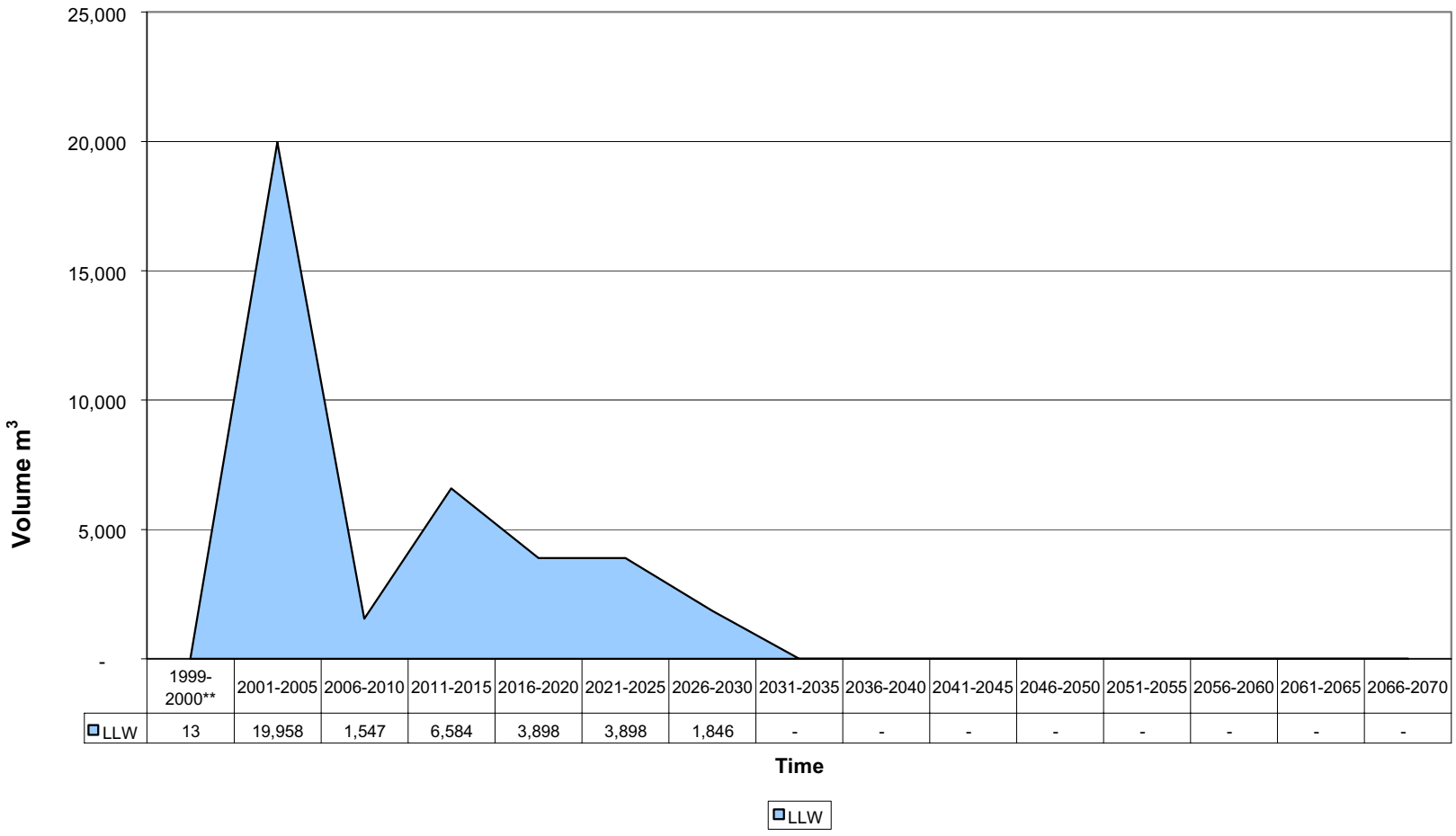

Total life-cycle values per 7/30/99 Stream Disposition Data (SDD) (http://id.inel.gov/avs/) 
Savannah River Site

Outgoing LLW Volume to Envirocare

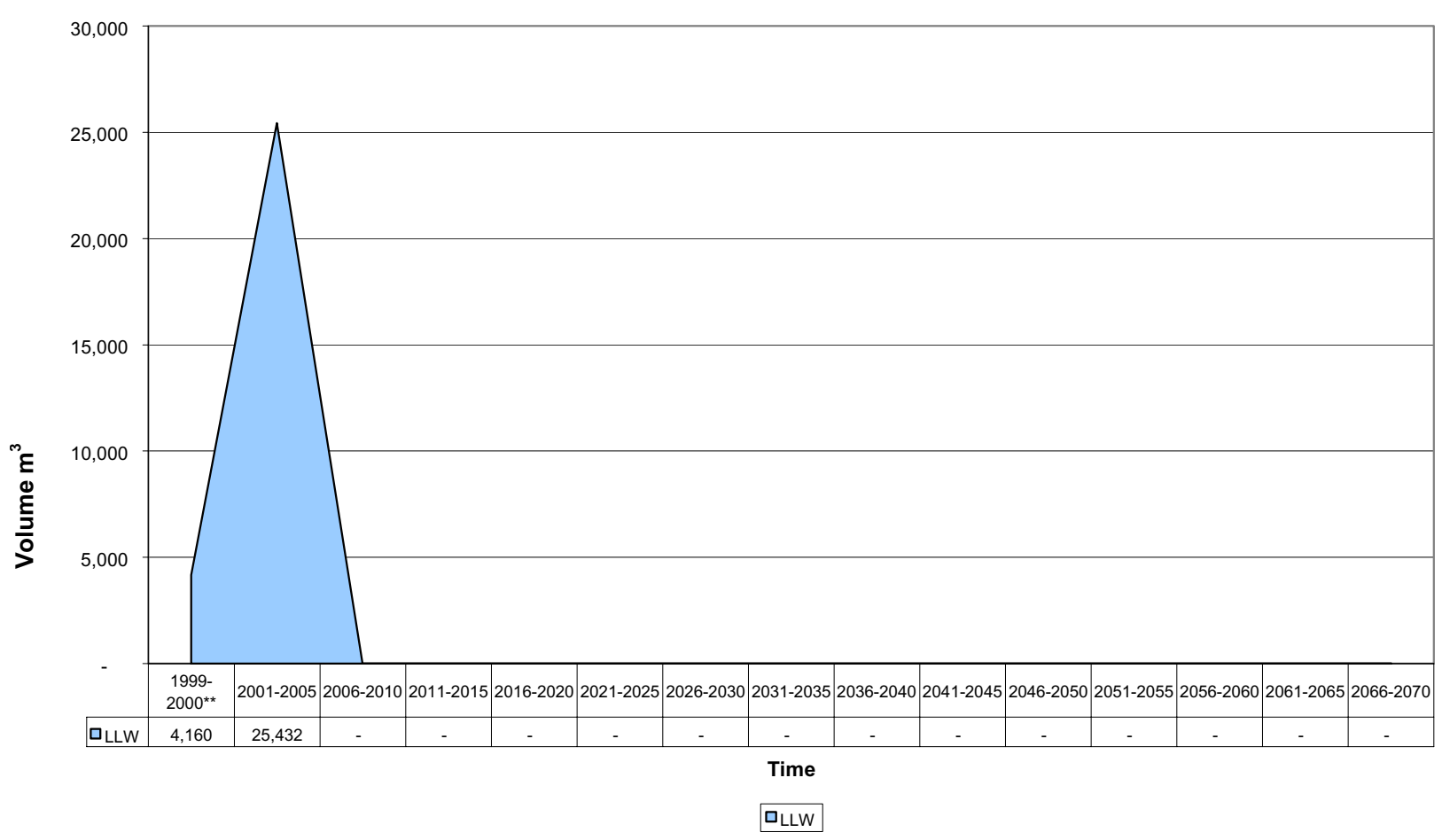

Savannah River Site

Waste Stream Disposition

Life-cycle Outgoing SNF

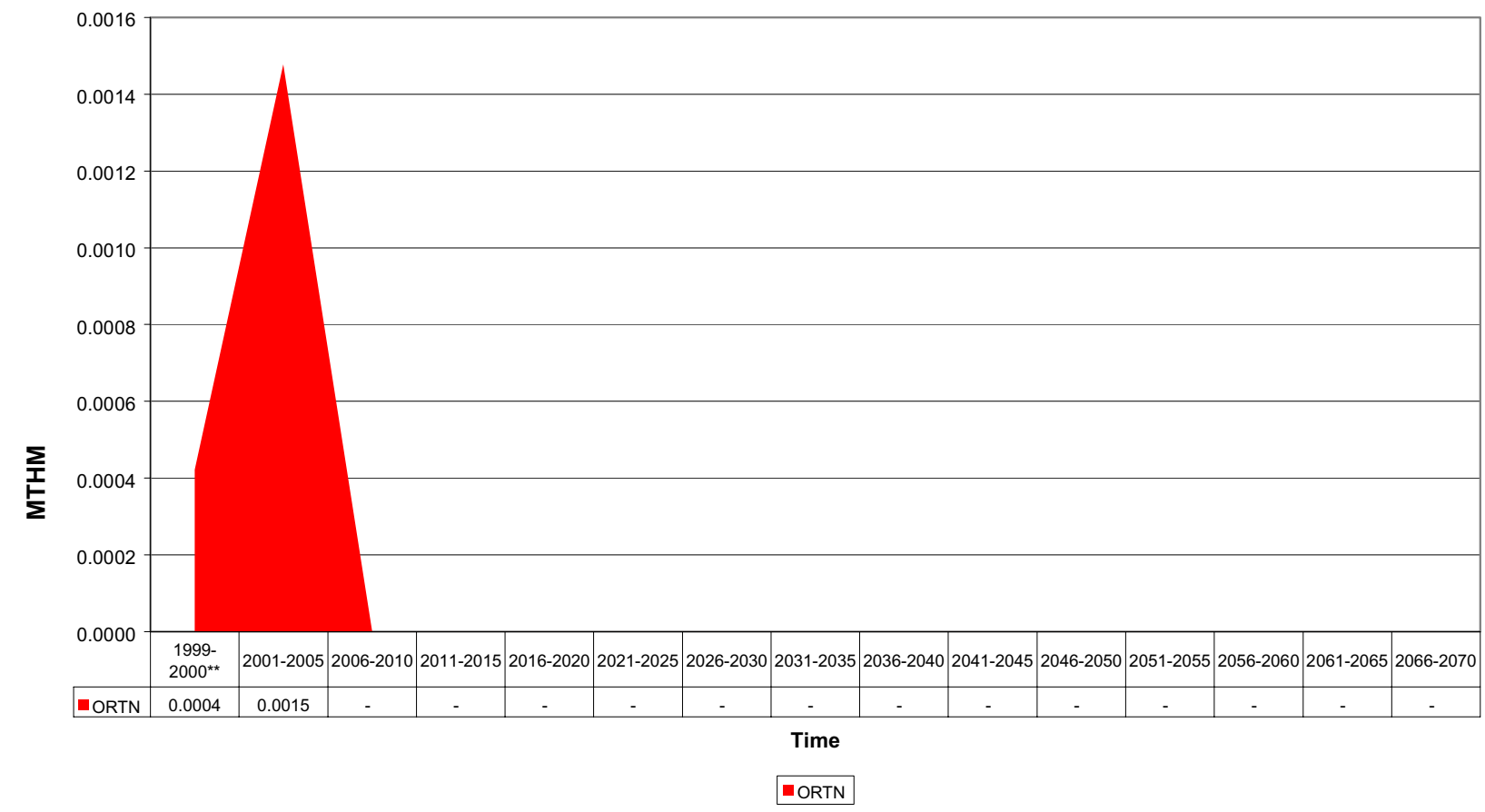

Total life-cycle values per 7/30/99 Stream Disposition Data (SDD) (http://id.inel.gov/avs/)

A-101

(and/or 1999 Integrated Repository Receipts Workshop for GRD data). Printed: March 30, 2000

** Represents data for a two year period. 
Savannah River Site

Outgoing SNF Shipments to Oak Ridge

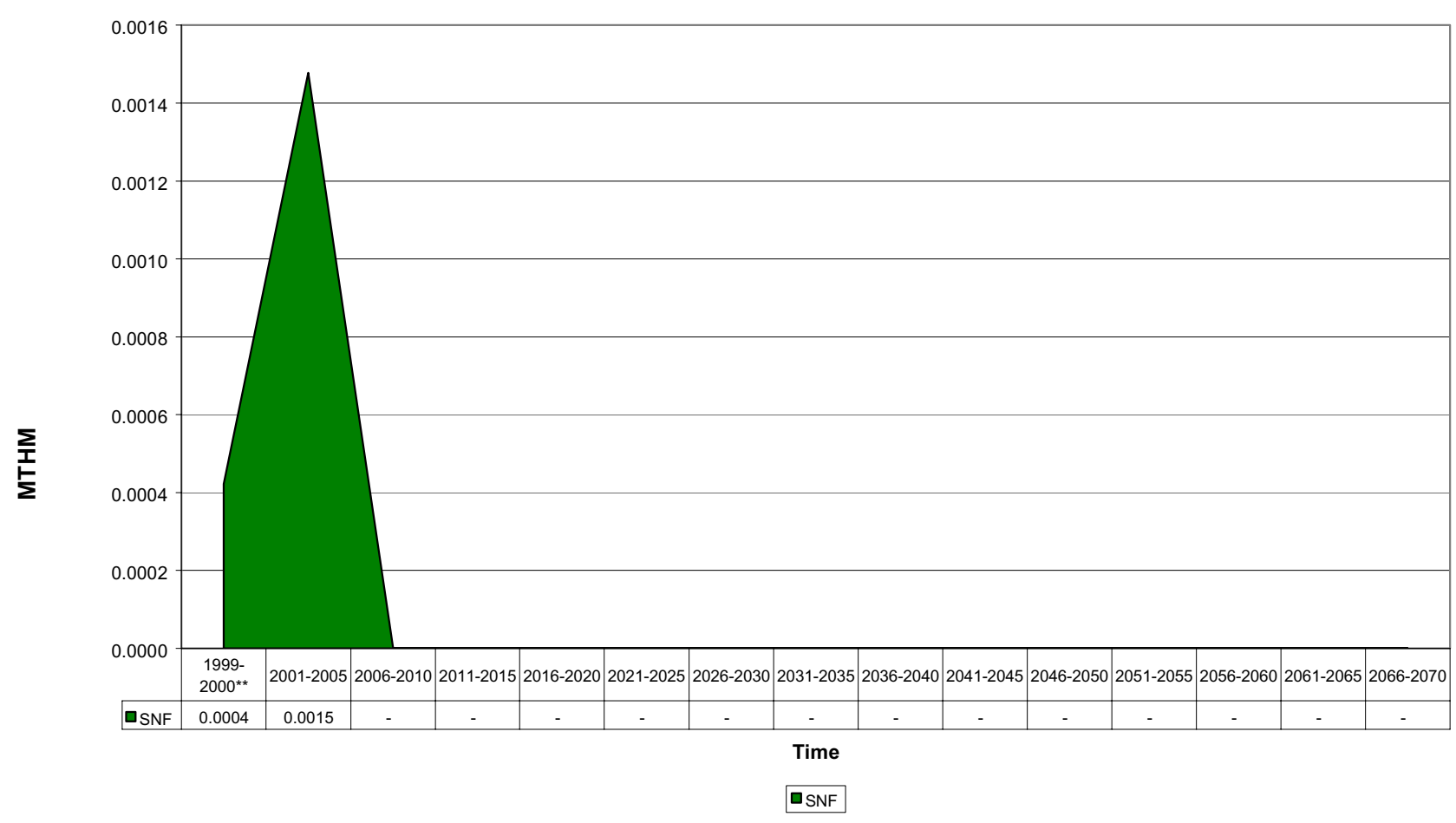

Total life-cycle values per 7/30/99 Stream Disposition Data (SDD) (http://id.inel.gov/avs/) 


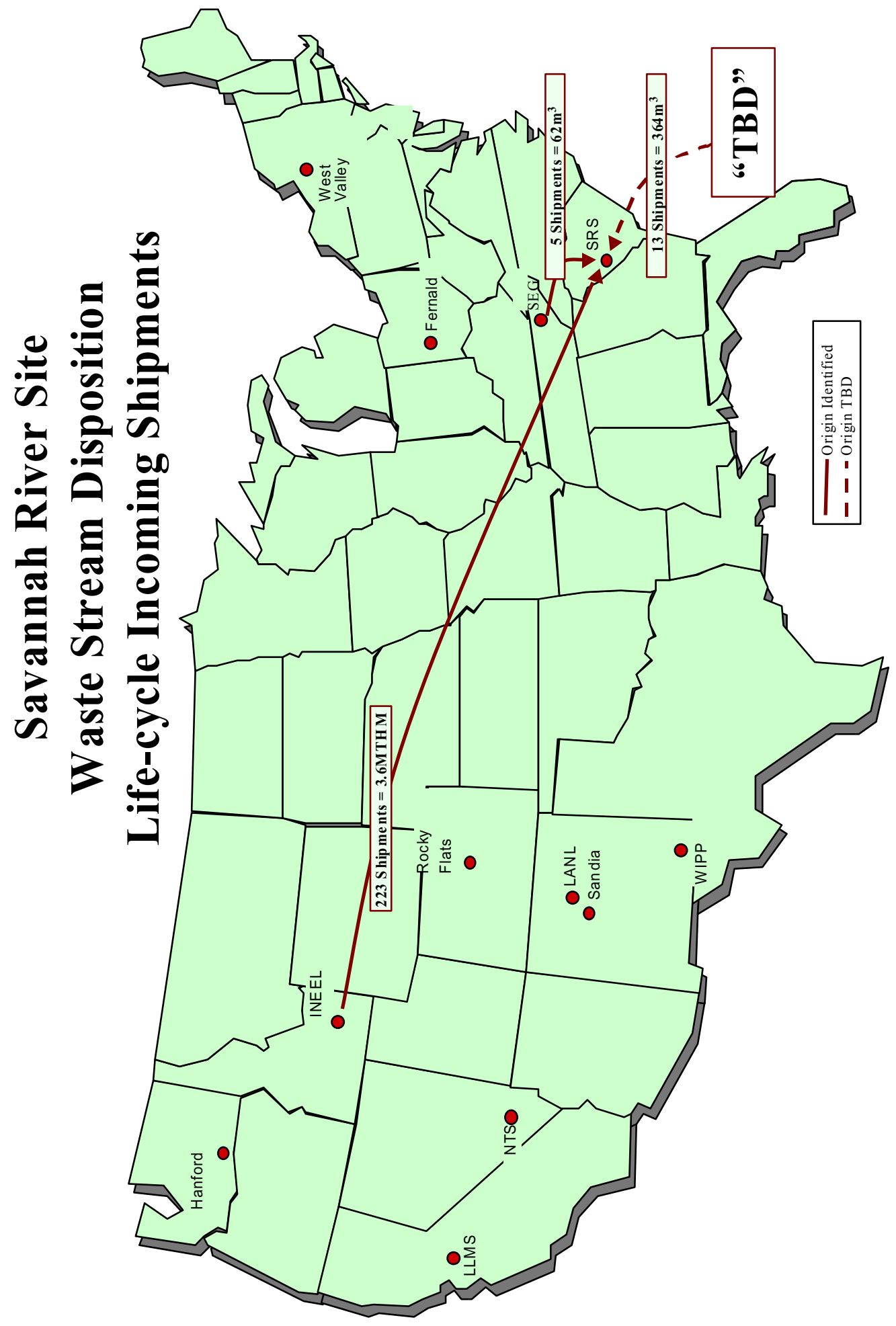


Savannah River Site

Waste Stream Disposition

Life-cycle Incoming Shipments

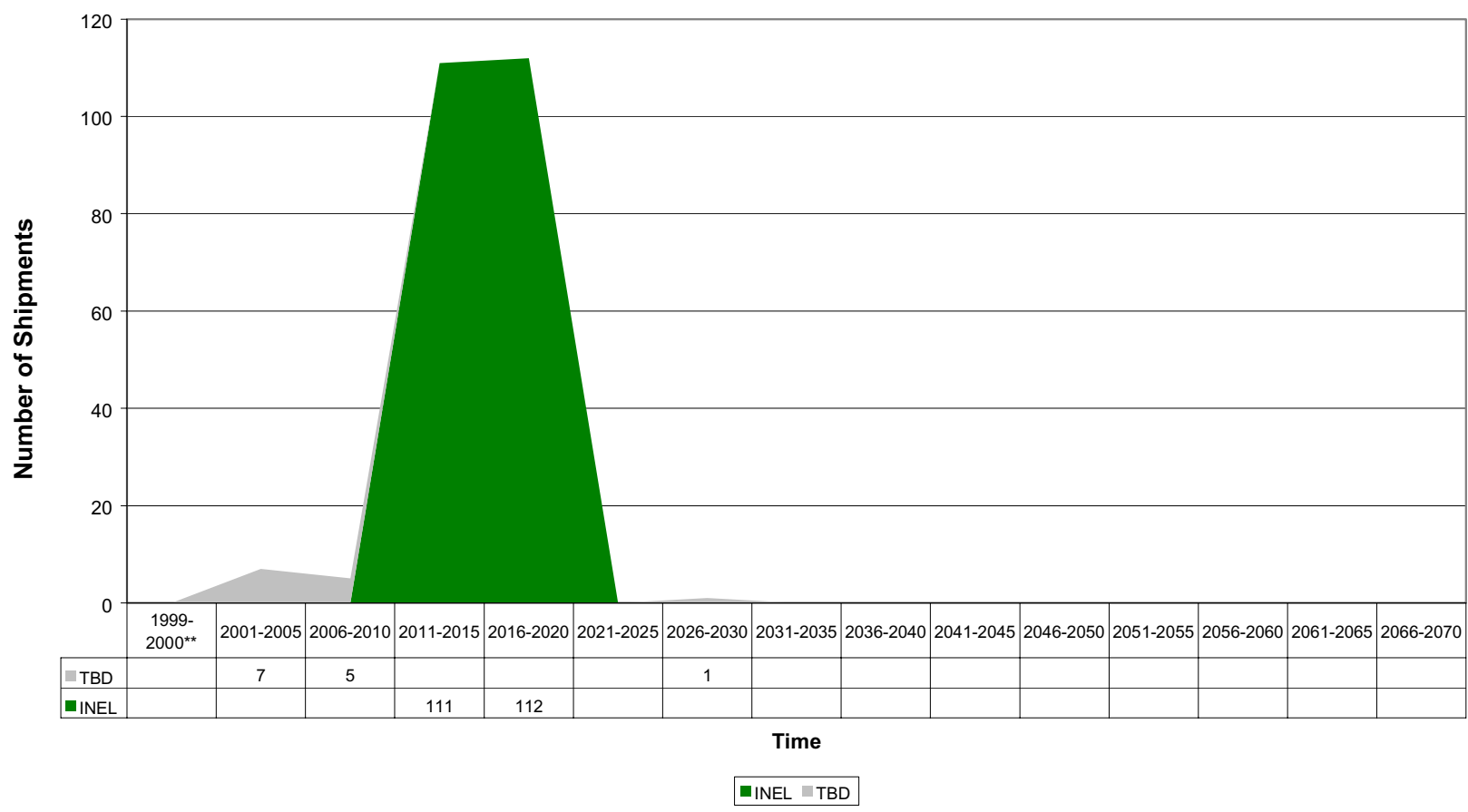

Savannah River Site

Incoming SNF Shipments from INEEL

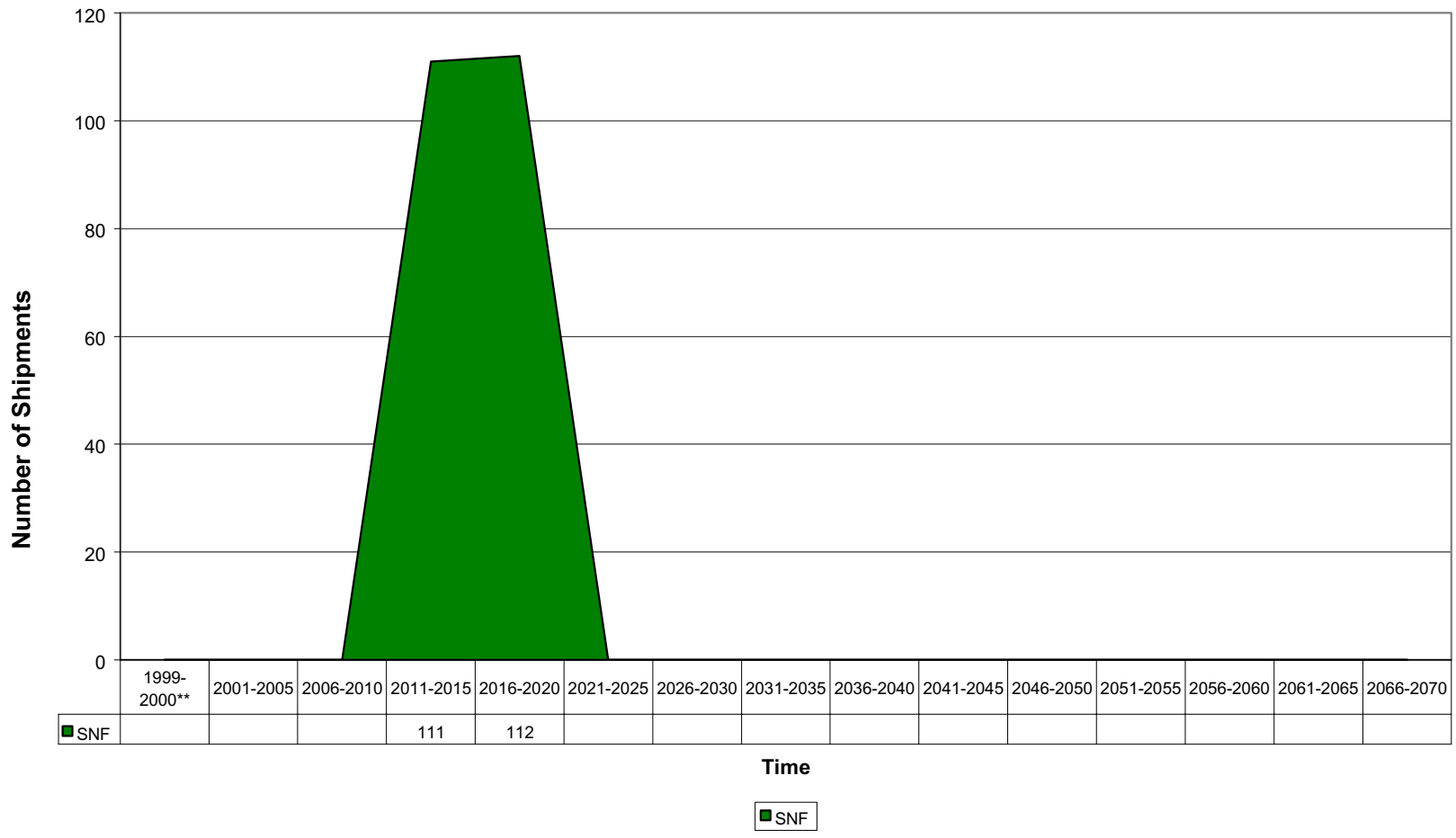

Total life-cycle values per 7/30/99 Stream Disposition Data (SDD) (http://id.inel.gov/avs/). Printed: March 30, 2000

** Represents data for a two year period. 
Savannah River Site

Incoming M/LLW Shipments from "TBD"

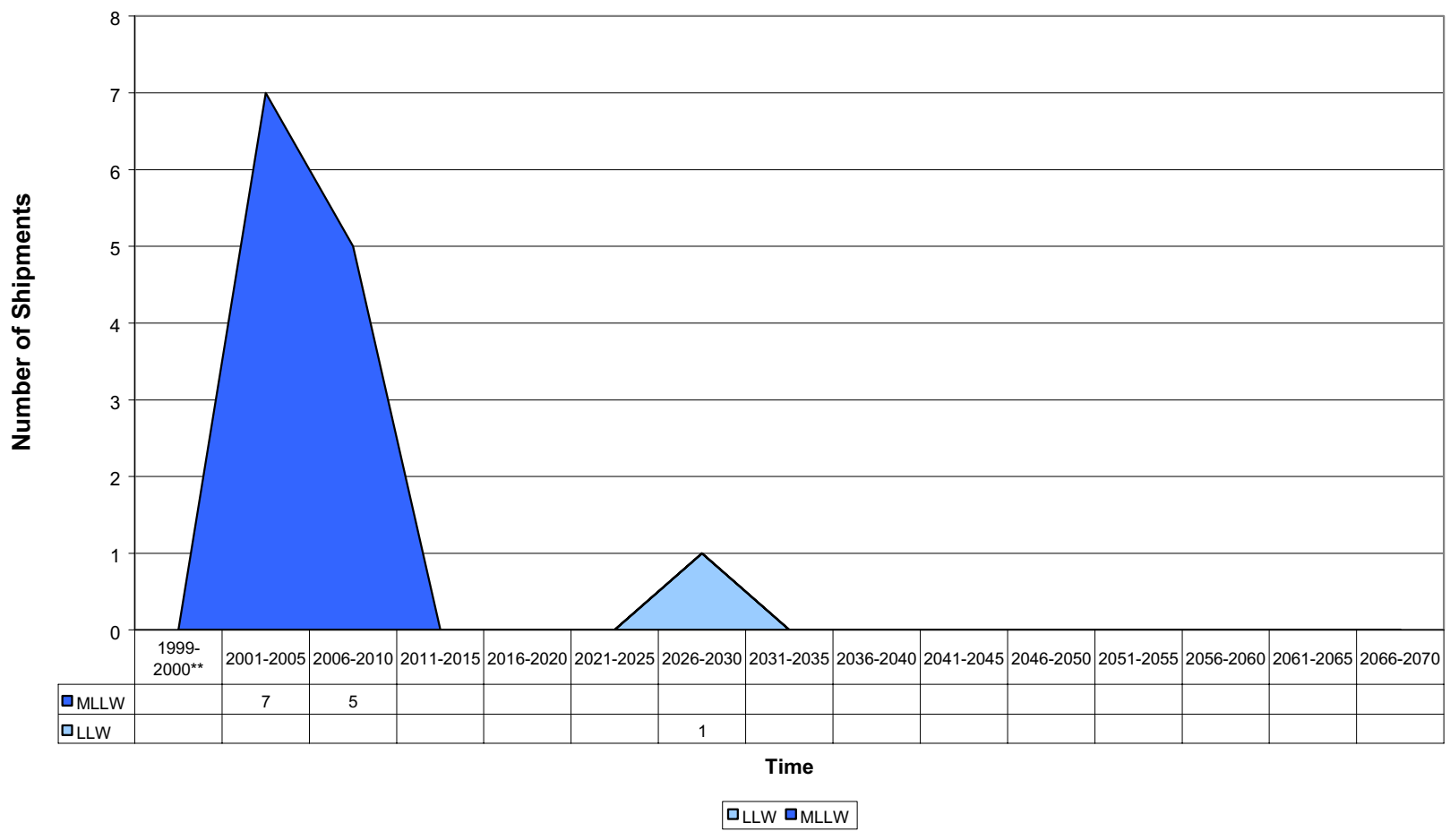




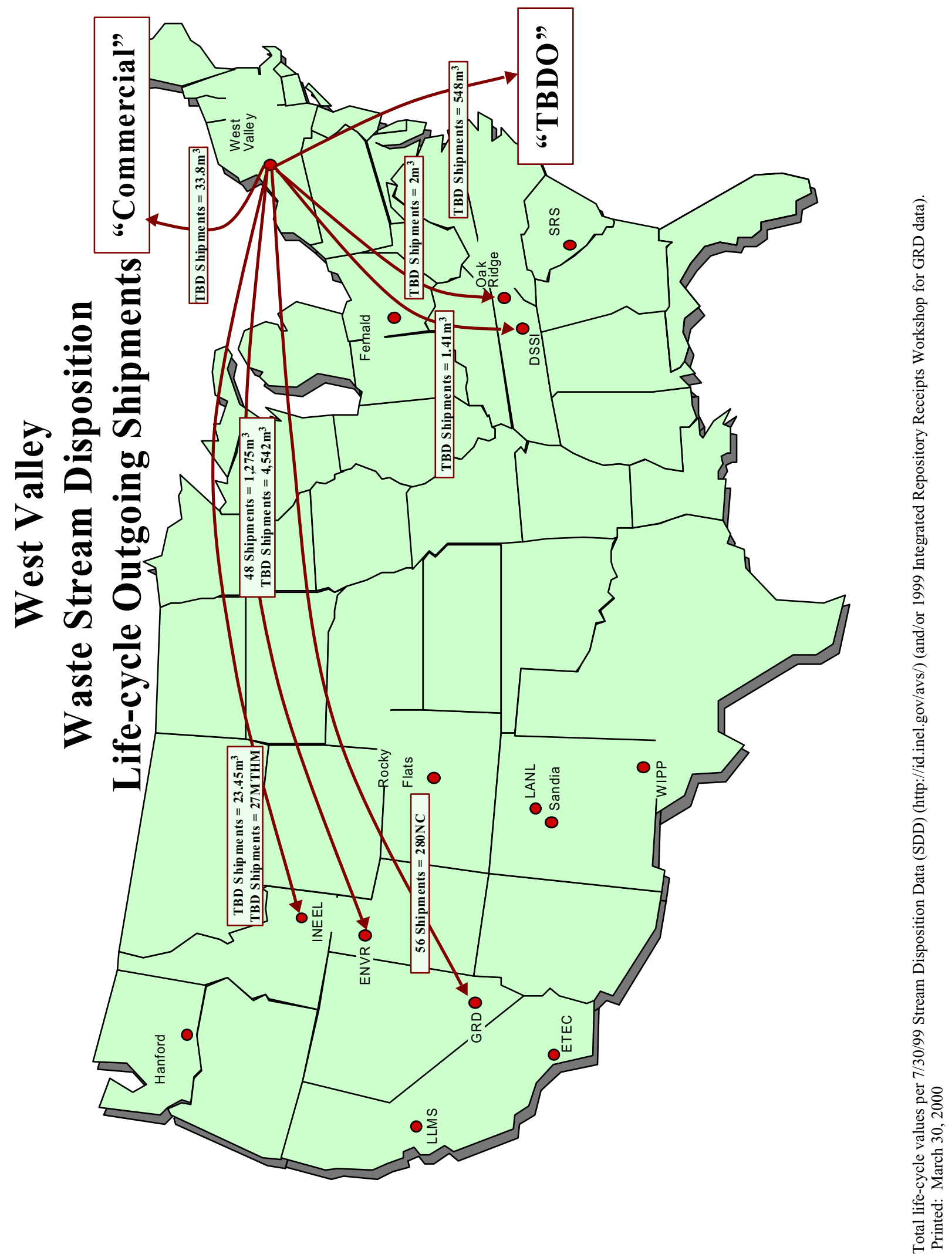
West Valley

Waste Stream Disposition

Life-cycle Outgoing Shipments

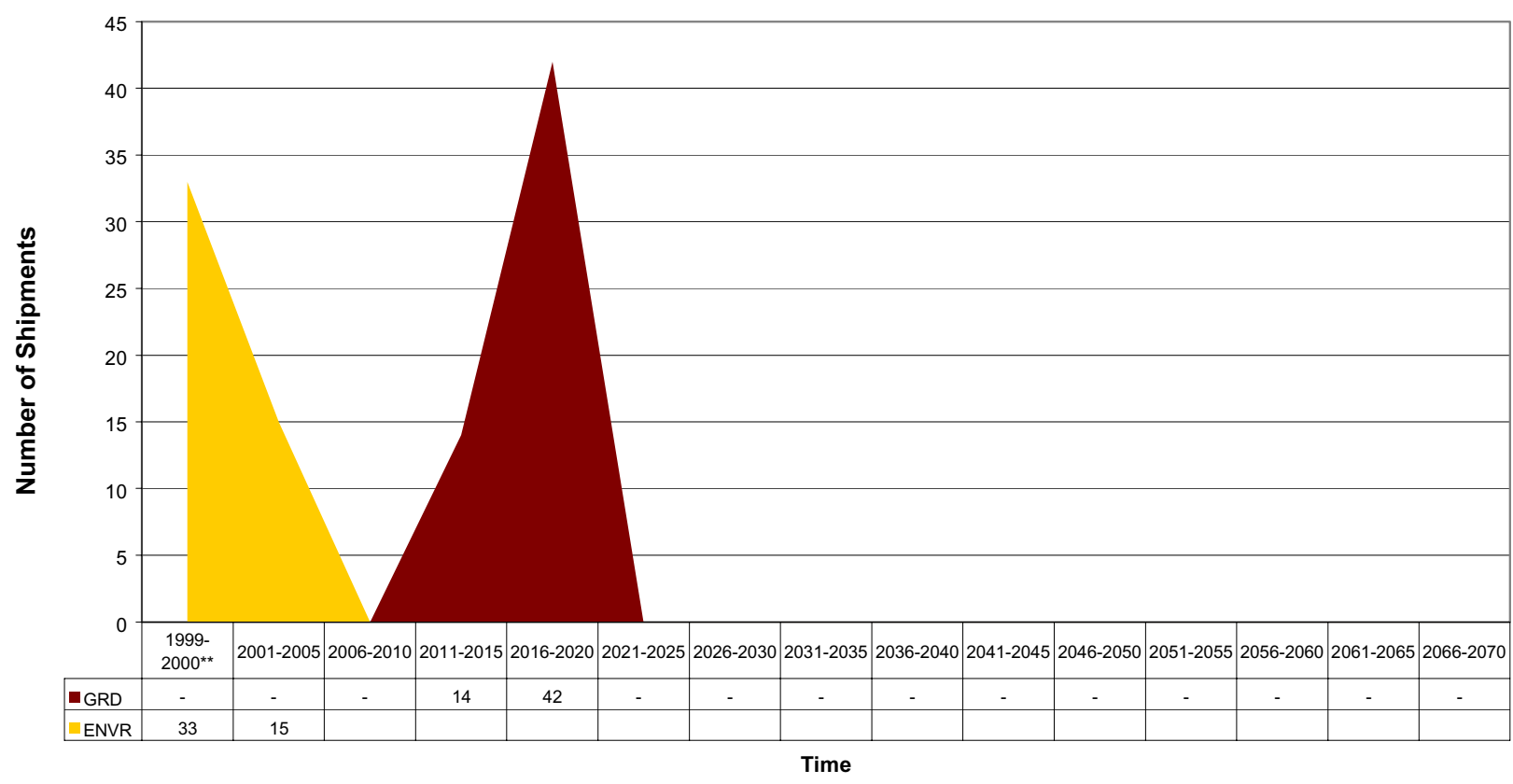

ENVR —GRD

West Valley

Outgoing LLW Shipments to Envirocare

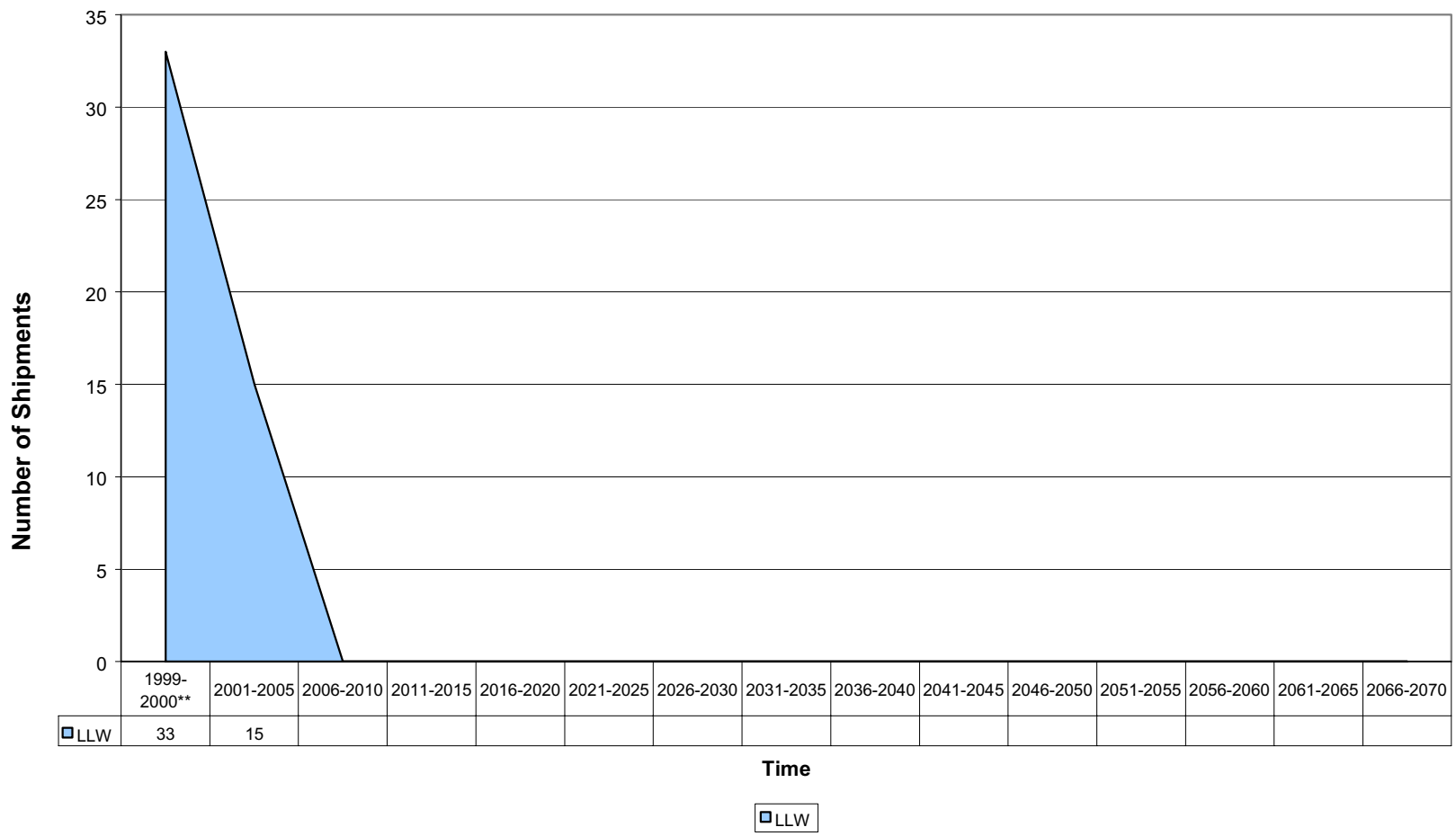

Total life-cycle values per 7/30/99 Stream Disposition Data (SDD) (http://id.inel.gov/avs/)

A-108 (and/or 1999 Integrated Repository Receipts Workshop for GRD data). Printed: March 30, 2000 ** Represents data for a two year period. 
West Valley

Outgoing HLW Shipments to Geologic Repository Disposal

(Tentatively Yucca Mtn.)

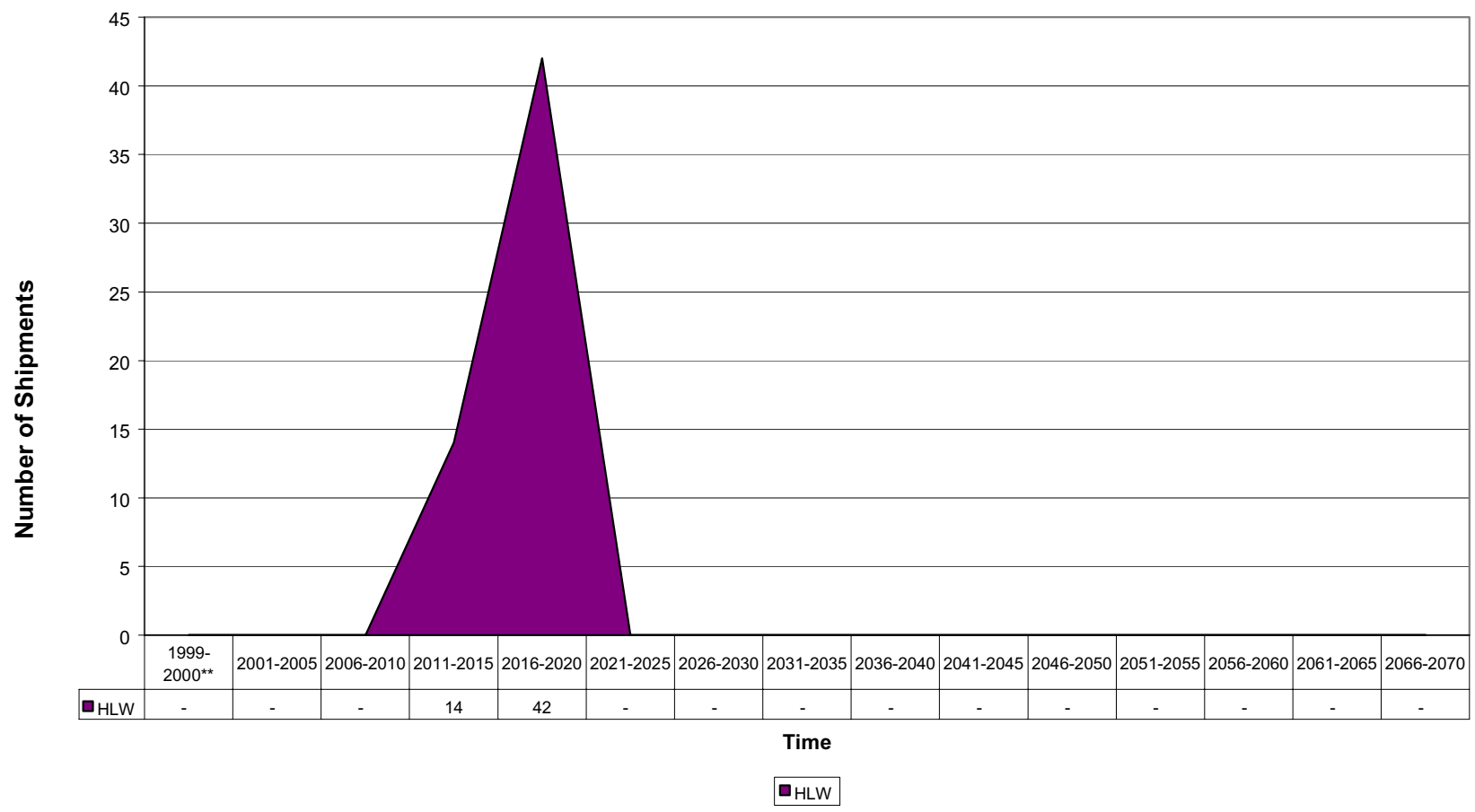

West Valley

Waste Stream Disposition

Life-cycle Outgoing Volume

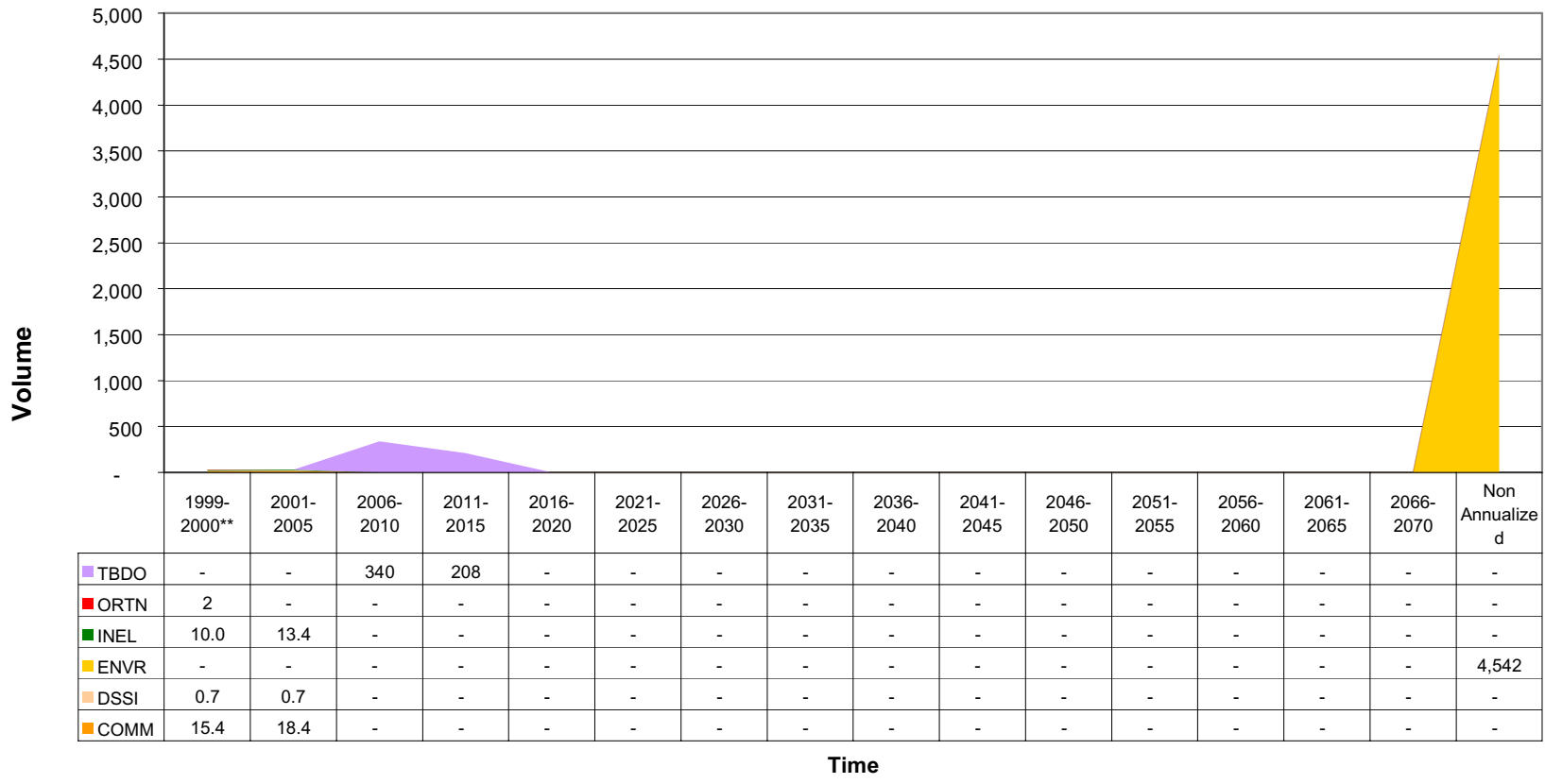

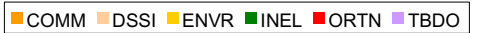

Total life-cycle values per 7/30/99 Stream Disposition Data (SDD) (http://id.inel.gov/avs/)

A-109

(and/or 1999 Integrated Repository Receipts Workshop for GRD data). Printed: March 30, 2000

** Represents data for a two year period. 
West Valley

Outgoing MLLW Volume to "Commercial"

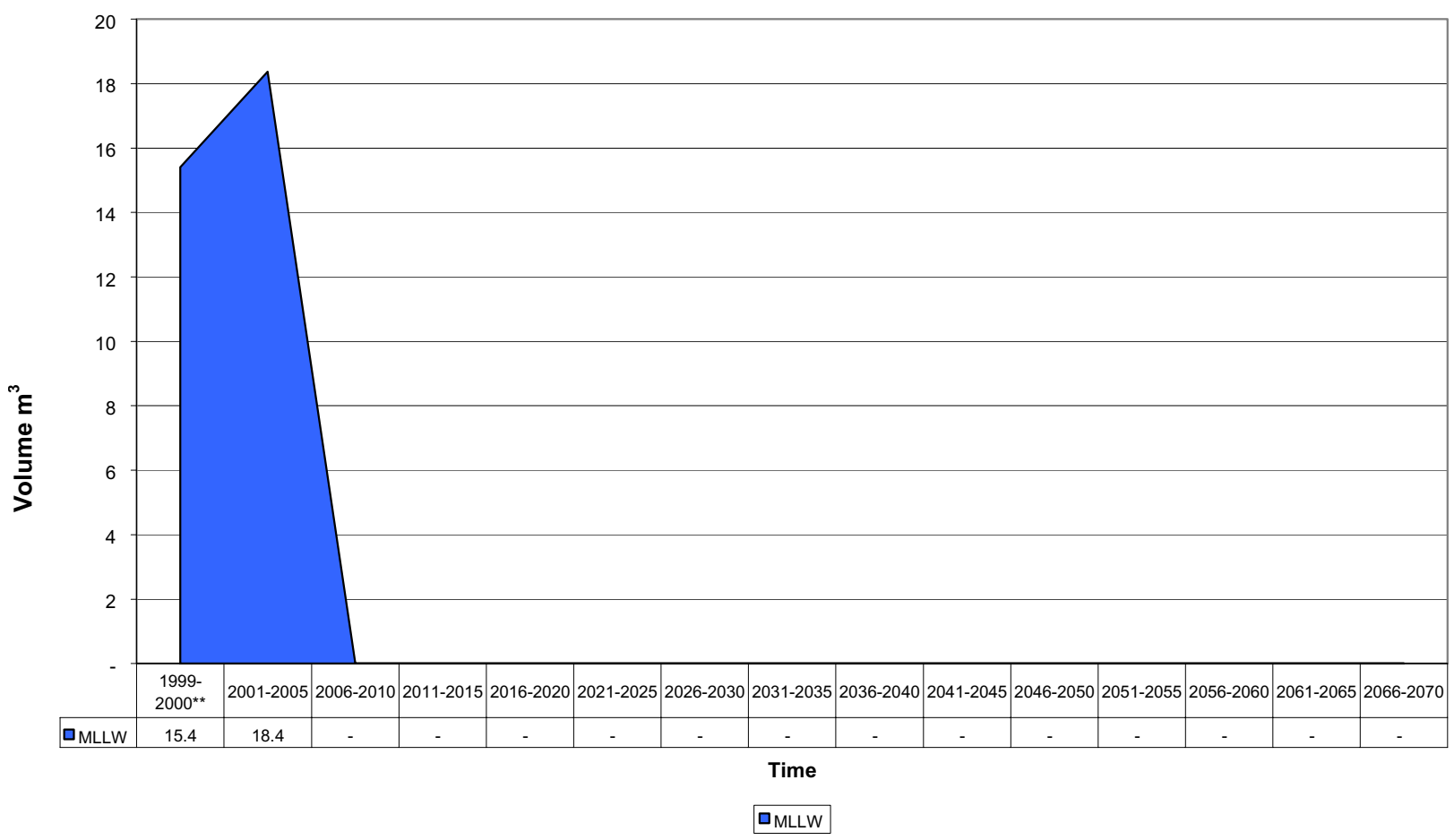

West Valley

Outgoing MLLW Volume to Diversified Scientific Services, Inc.

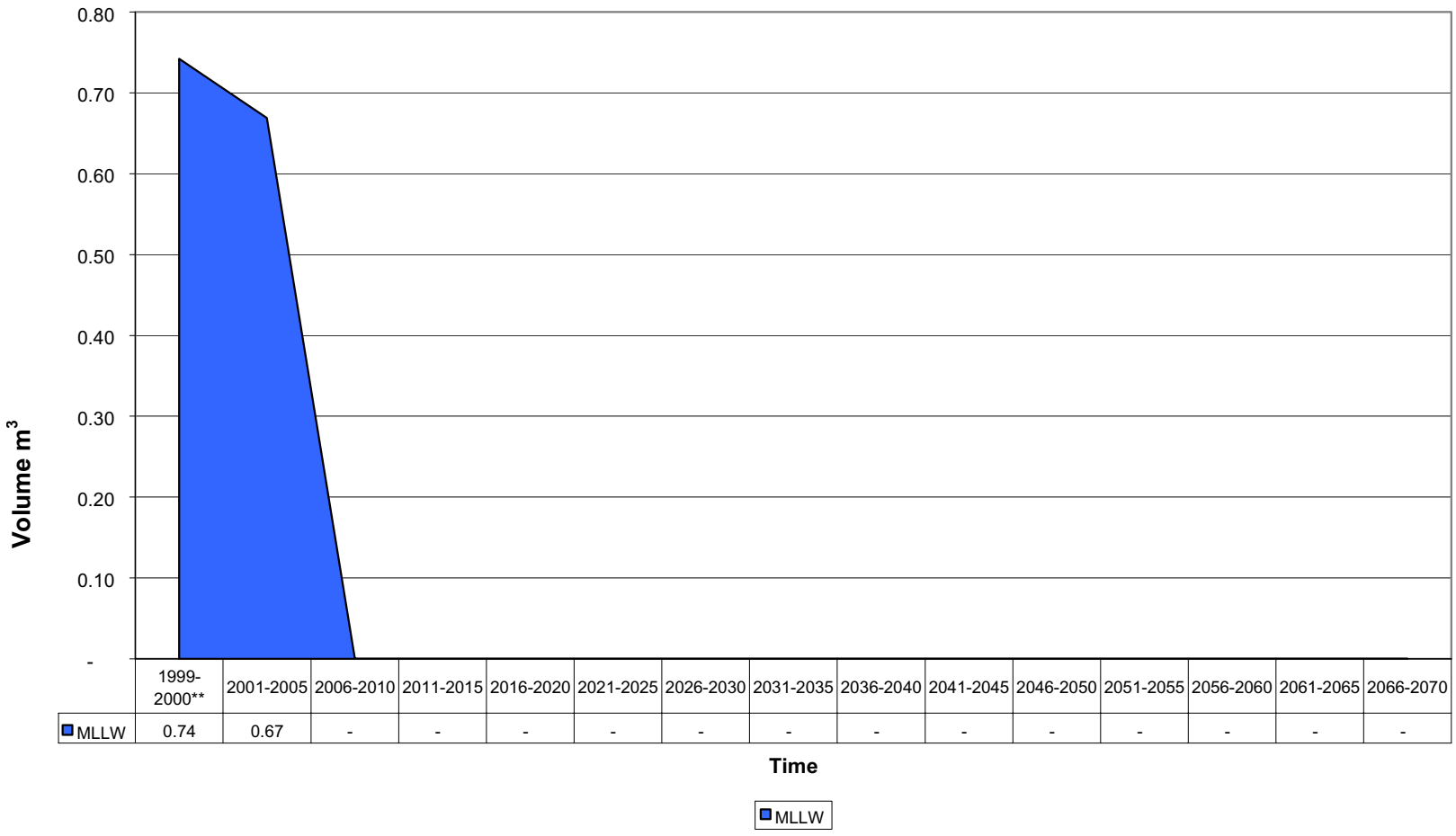

Total life-cycle values per 7/30/99 Stream Disposition Data (SDD) (http://id.inel.gov/avs/) 
West Valley

Outgoing LLW Volume to Envirocare

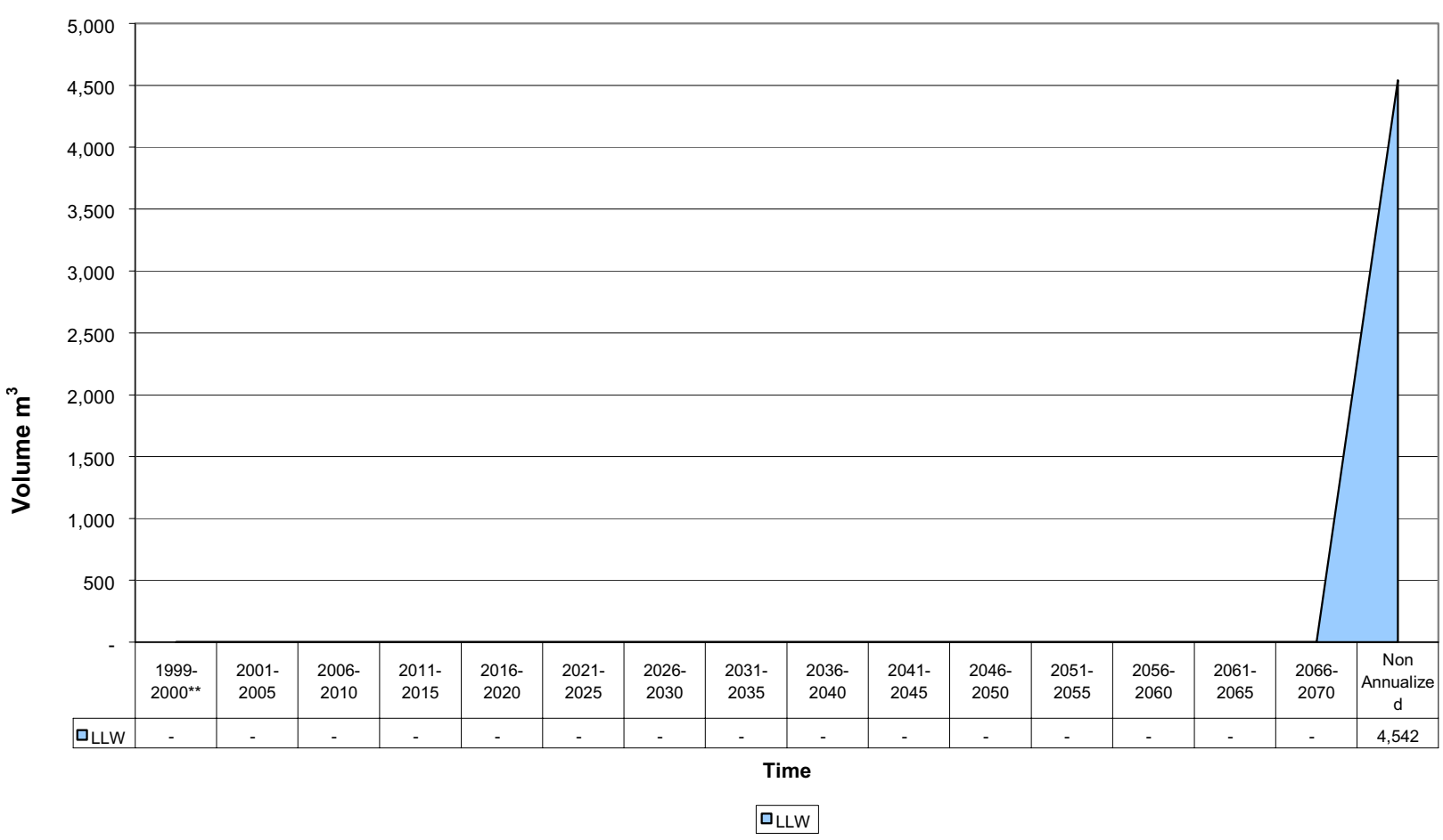

West Valley

Outgoing MLLW Volume to INEEL

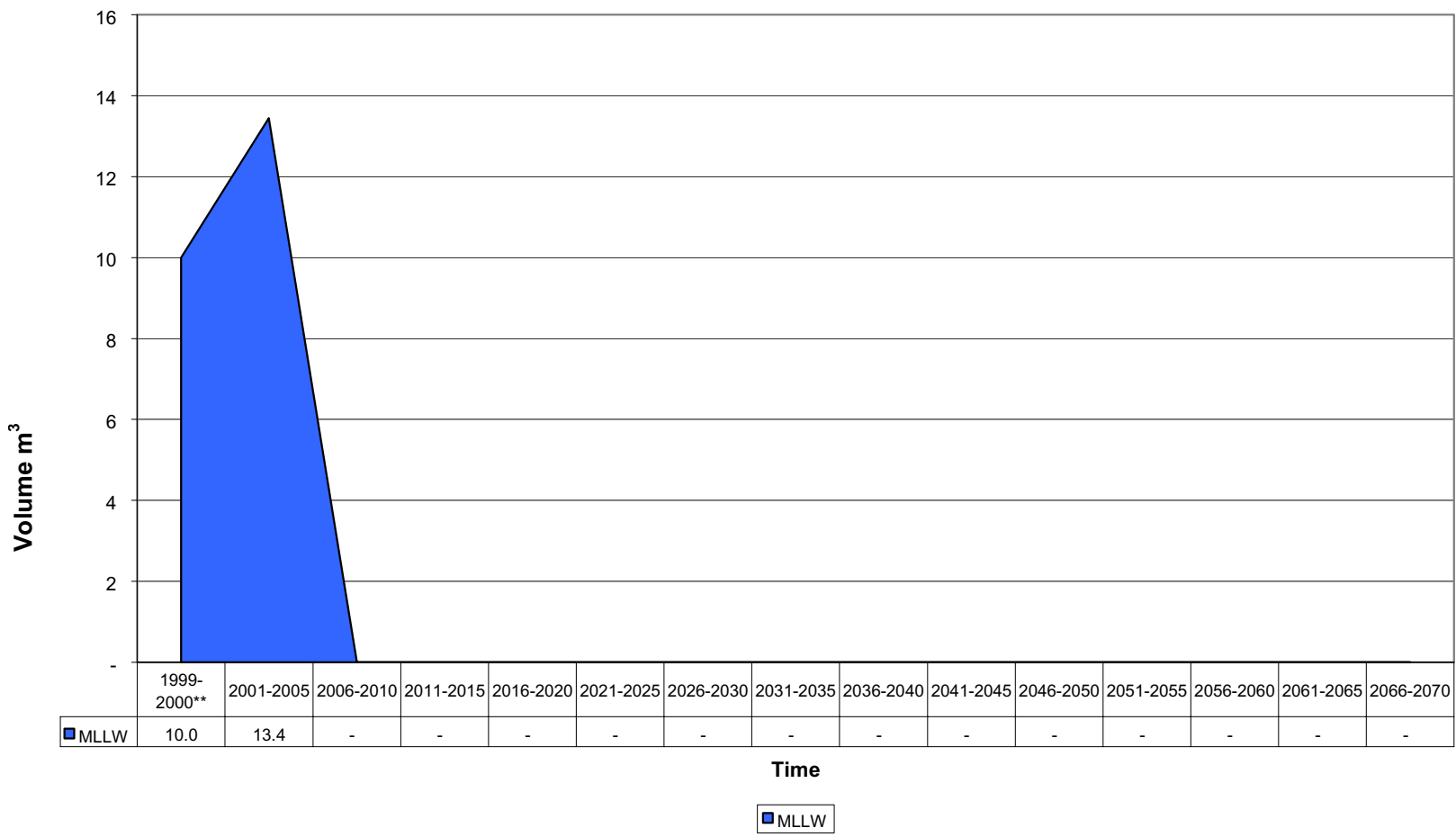

Total life-cycle values per 7/30/99 Stream Disposition Data (SDD) (http://id.inel.gov/avs/) 
West Valley

Outgoing MLLW Volume to Oak Ridge

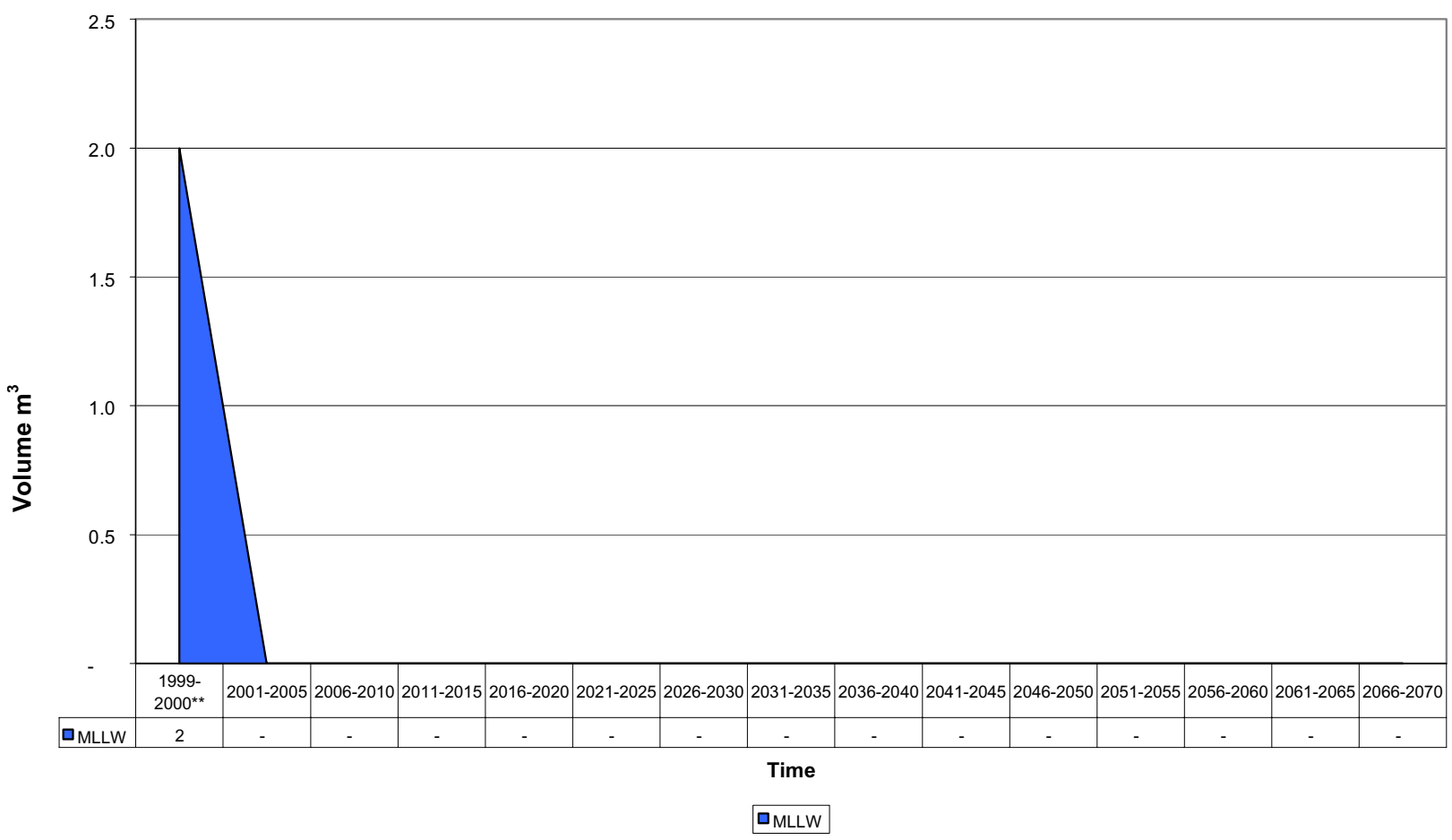

West Valley

Outgoing TRU Volume to "TBDO"

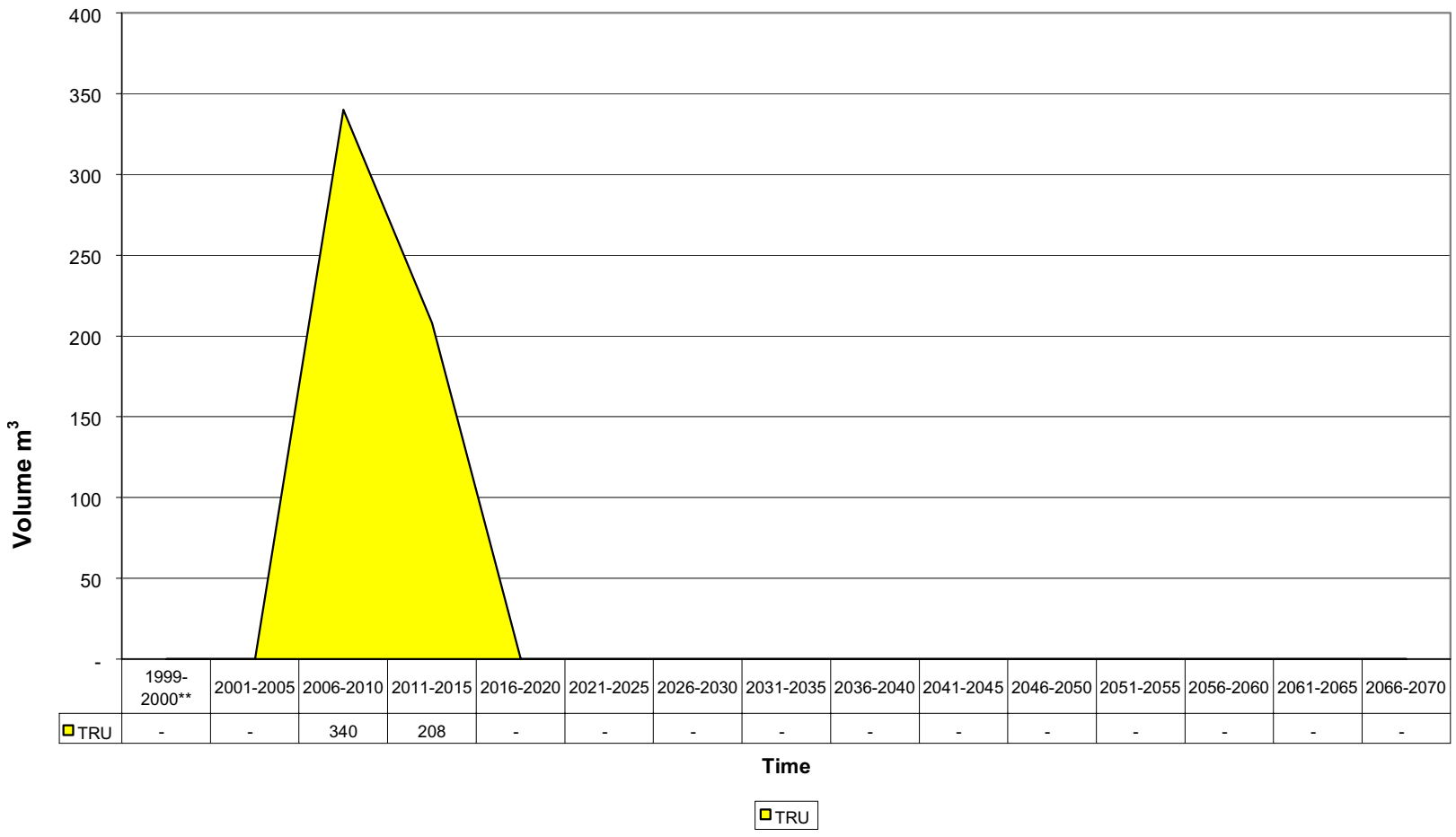

Total life-cycle values per 7/30/99 Stream Disposition Data (SDD) (http://id.inel.gov/avs/)

A-112

(and/or 1999 Integrated Repository Receipts Workshop for GRD data). Printed: March 30, 2000

** Represents data for a two year period. 
West Valley

Waste Stream Disposition Life-cycle Outgoing SNF

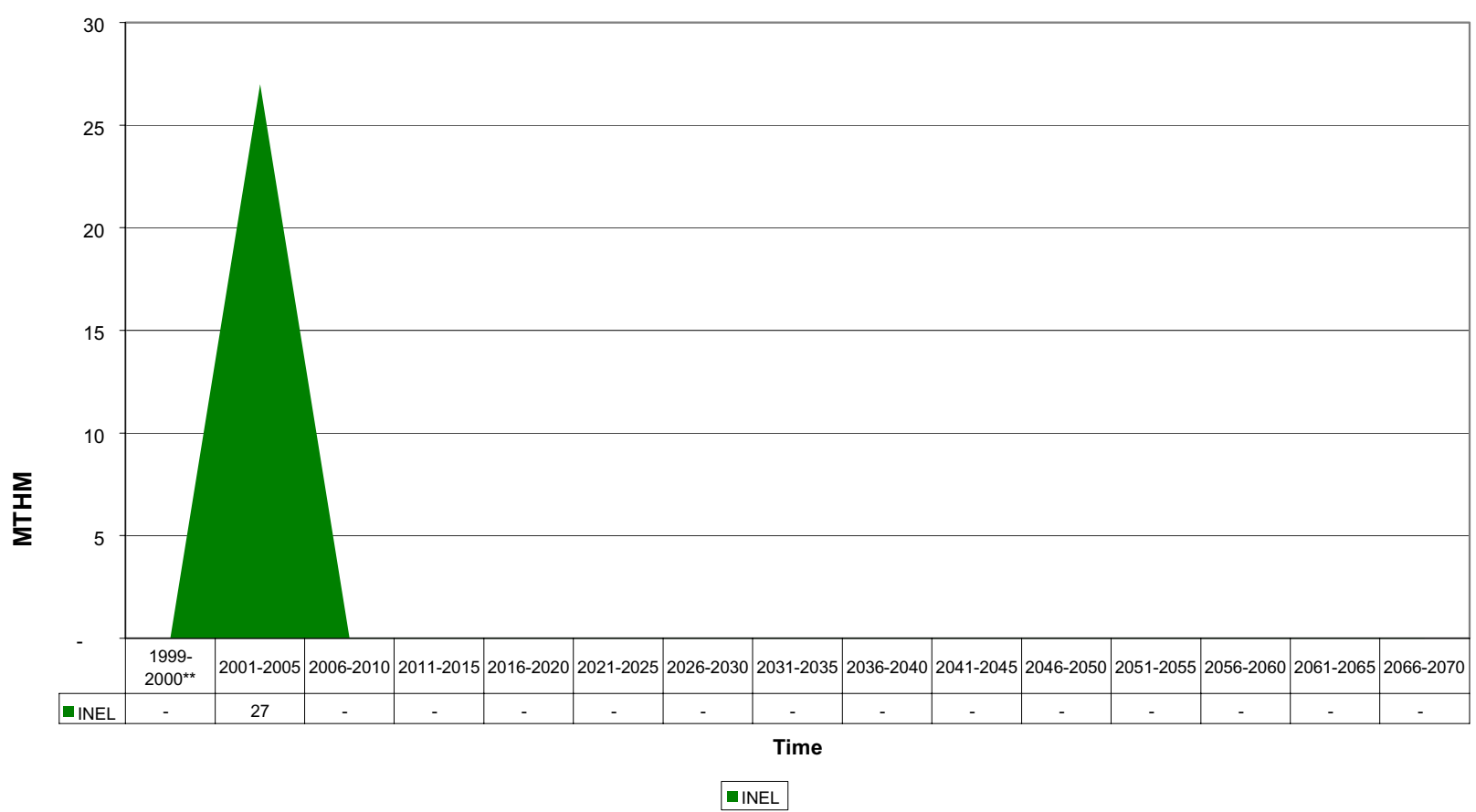

West Valley

Outgoing SNF to INEEL

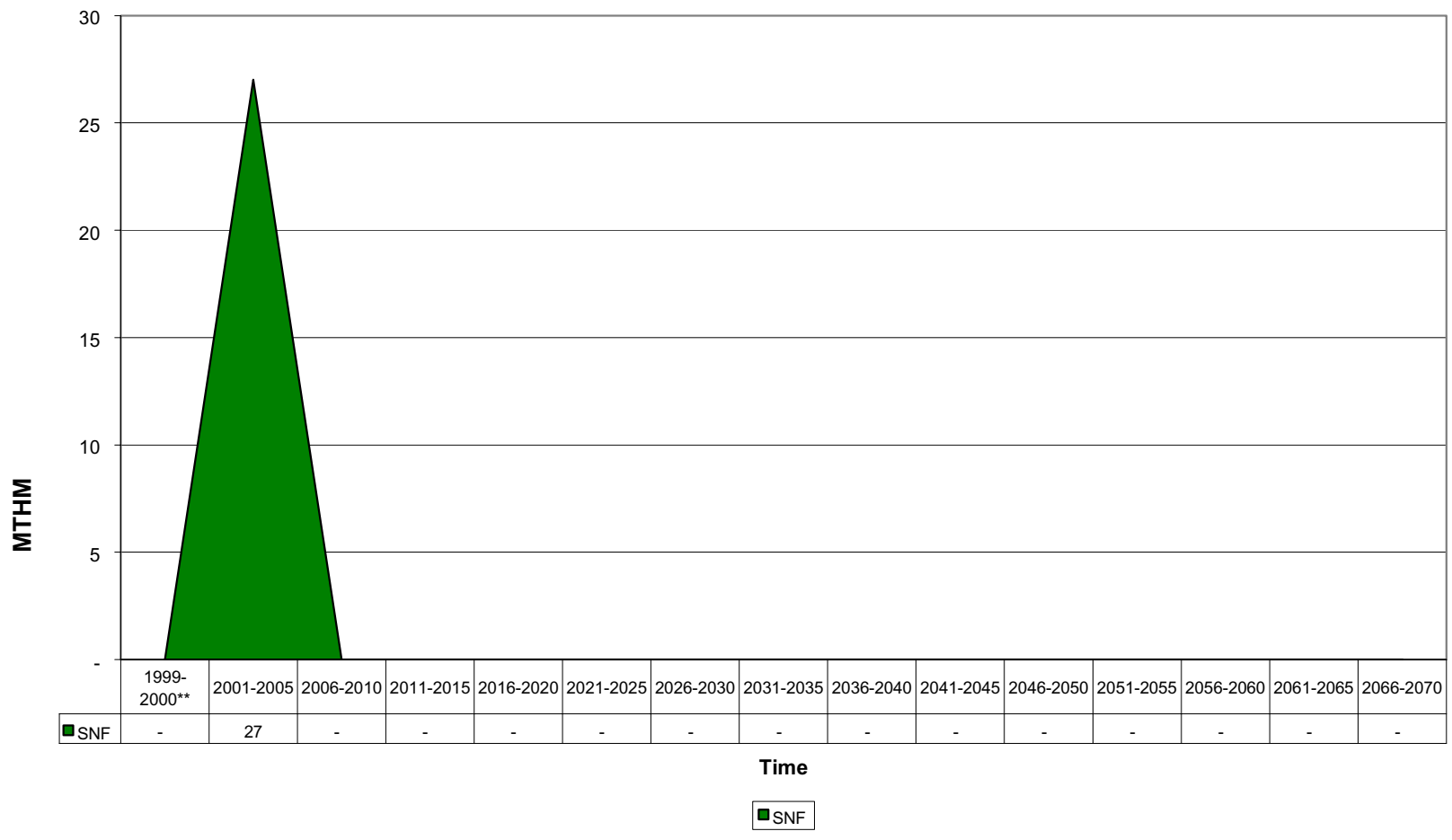

Total life-cycle values per 7/30/99 Stream Disposition Data (SDD) (http://id.inel.gov/avs/)

A-113

(and/or 1999 Integrated Repository Receipts Workshop for GRD data). Printed: March 30, 2000

** Represents data for a two year period. 



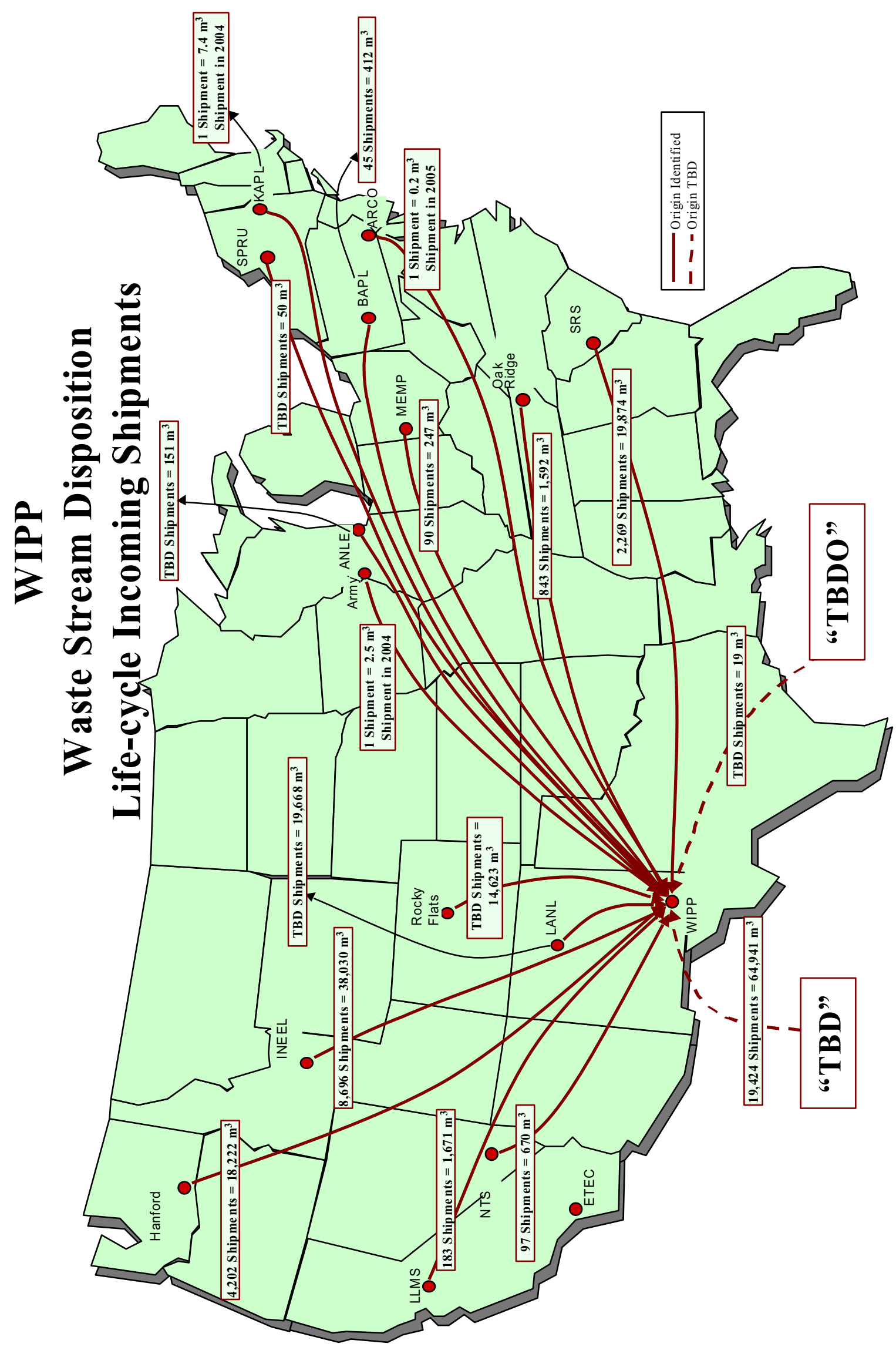

$\frac{n}{4}$

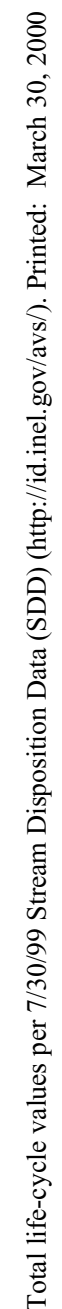


WIPP

Waste Stream Disposition Life-cycle Incoming Shipments

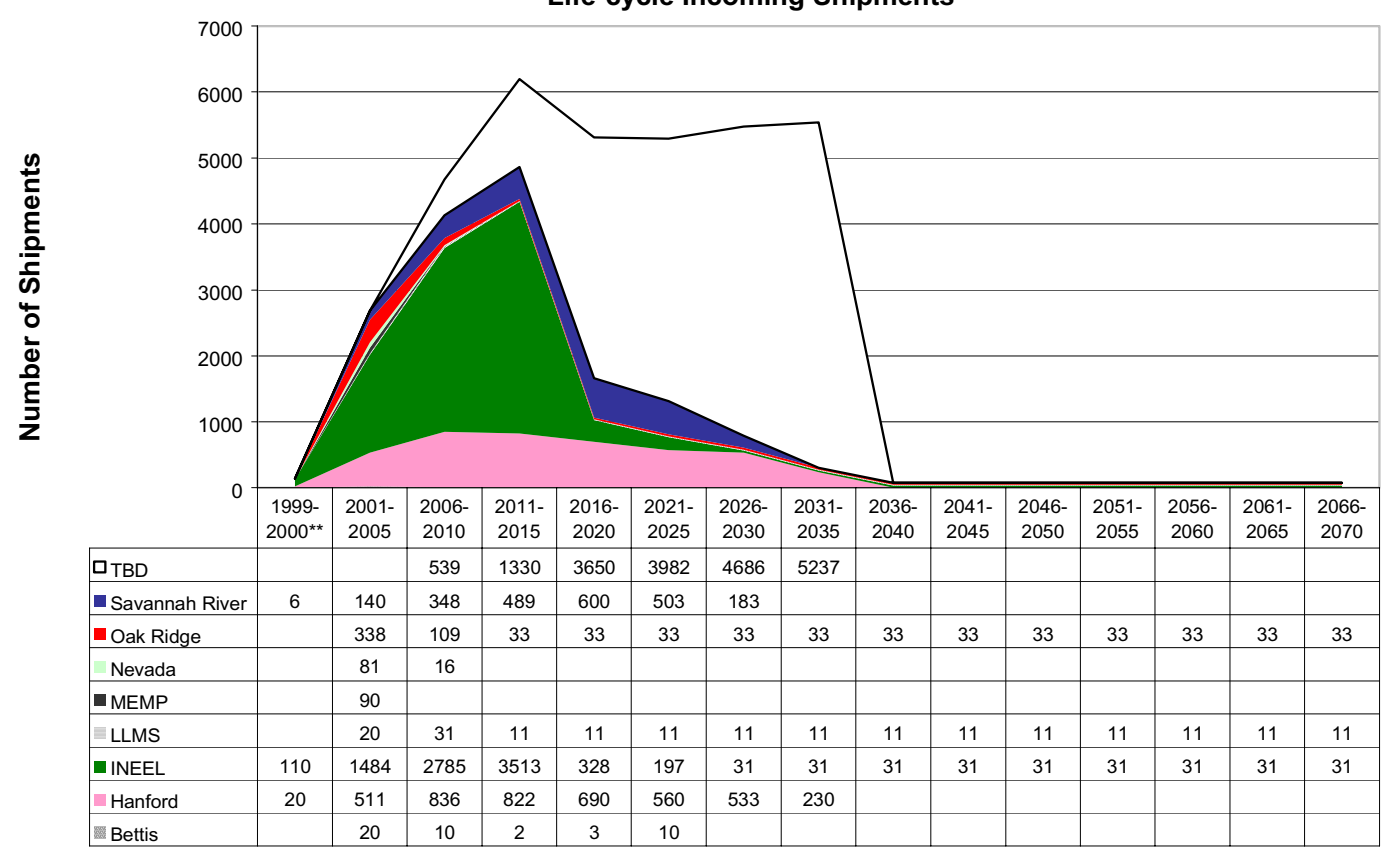

Time

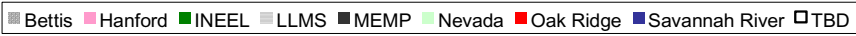

WIPP

Incoming TRU Shipments from INEEL

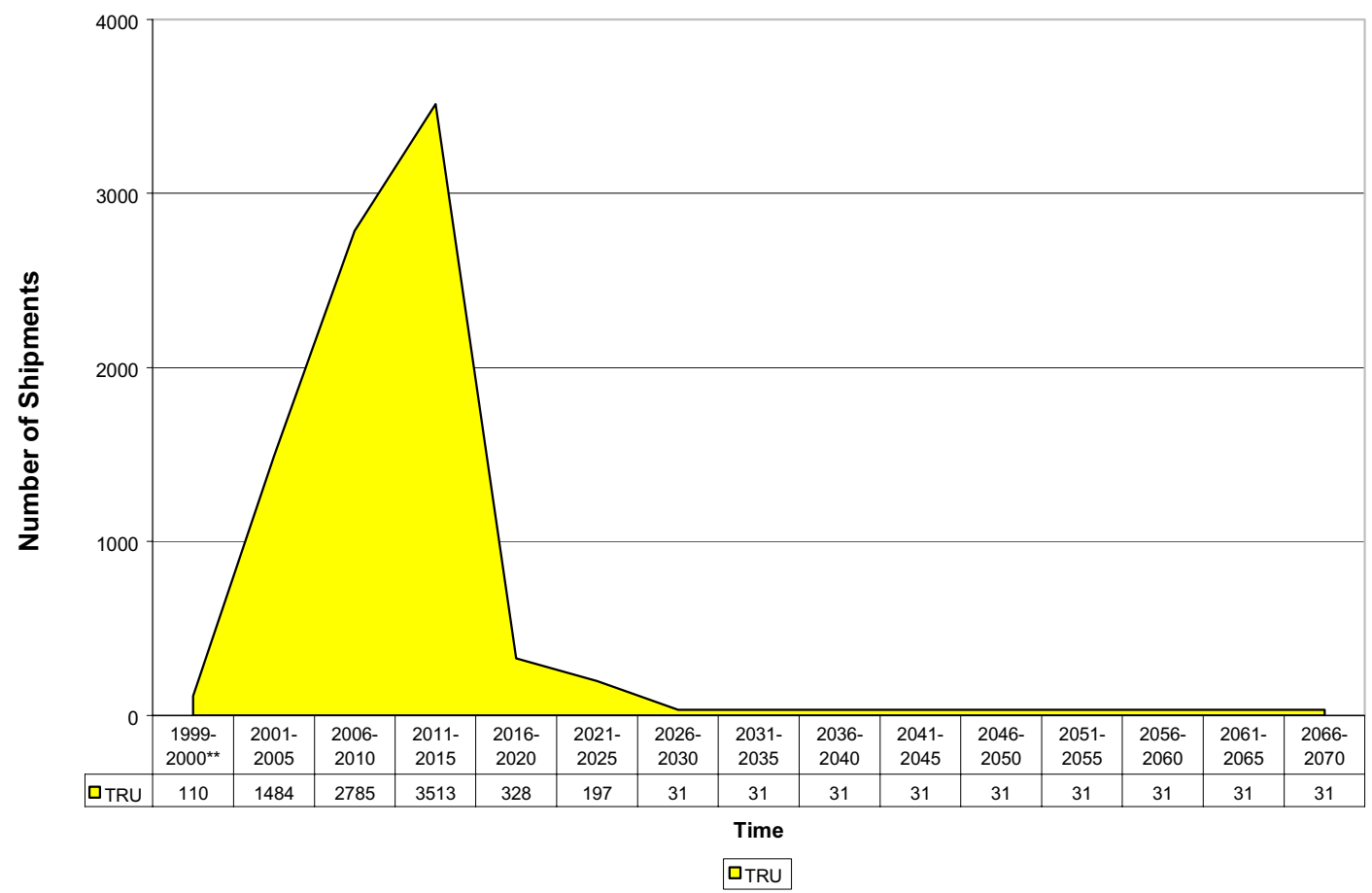

Total life-cycle values per 7/30/99 Stream Disposition Data (SDD) (http://id.inel.gov/avs/).

** Represents data for a two year period. 


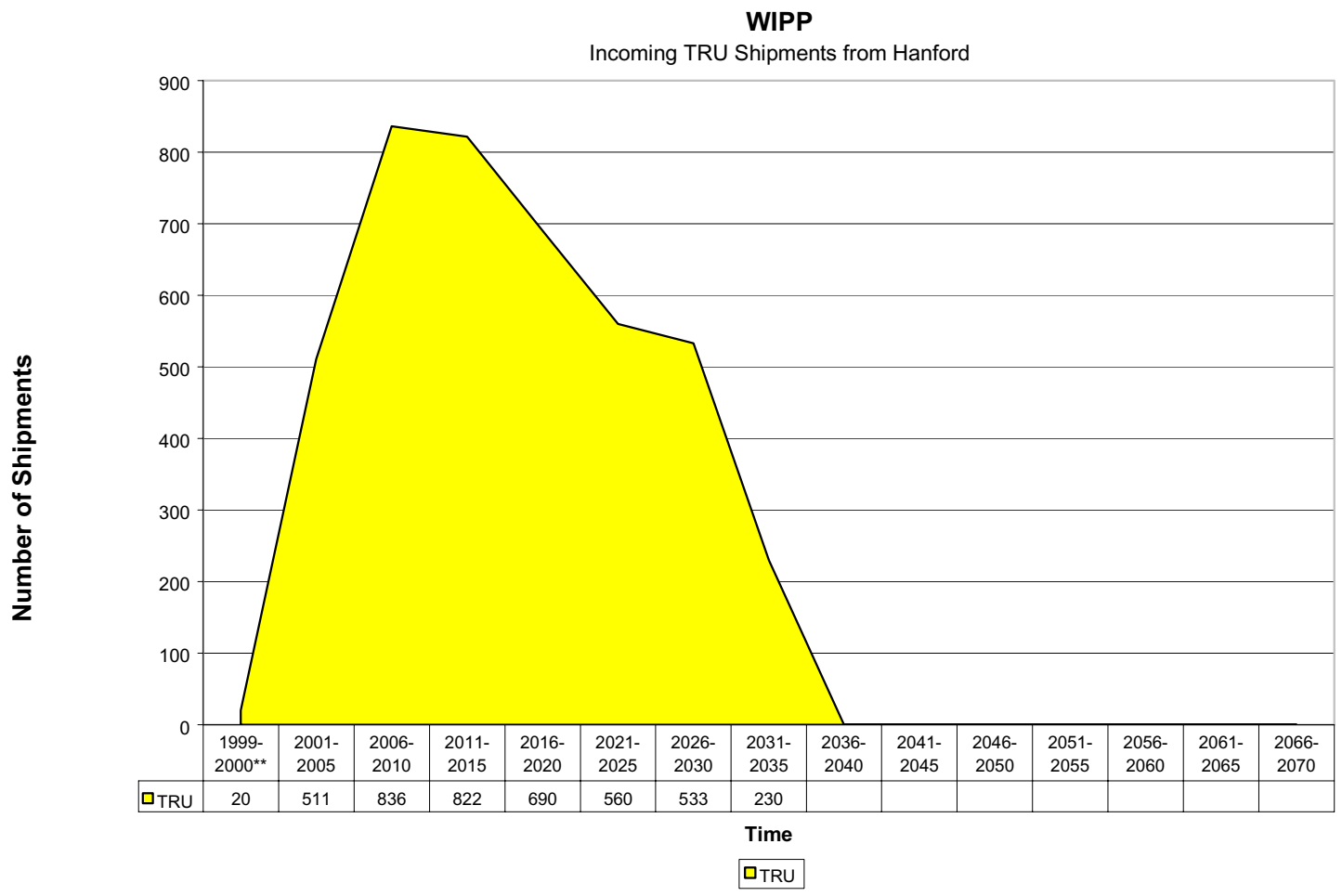

WIPP

Incoming TRU Shipments from

Lawrence Livermore National Lab - Main Site

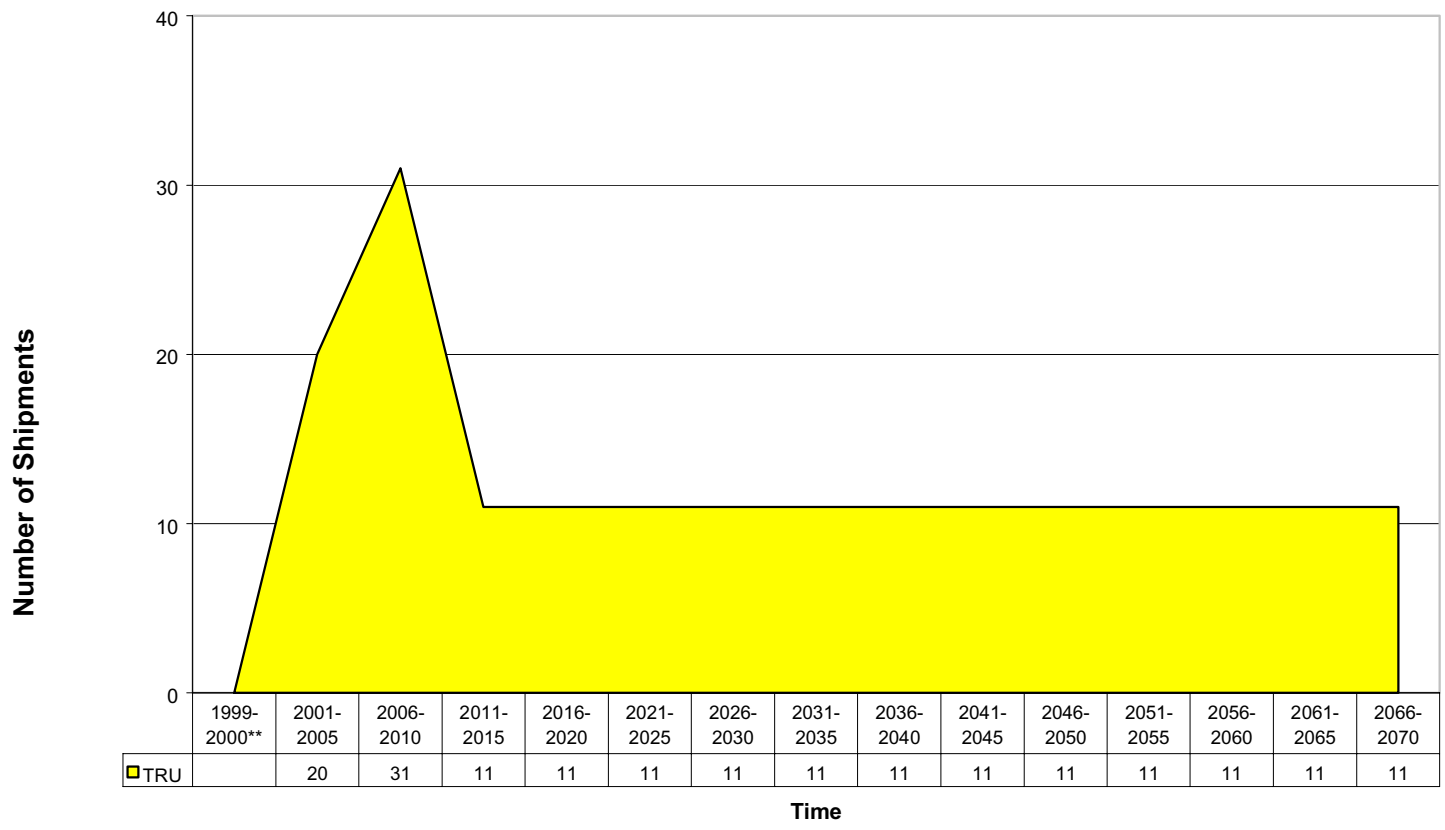

口TRU

Total life-cycle values per 7/30/99 Stream Disposition Data (SDD) (http://id.inel.gov/avs/).

** Represents data for a two year period. 

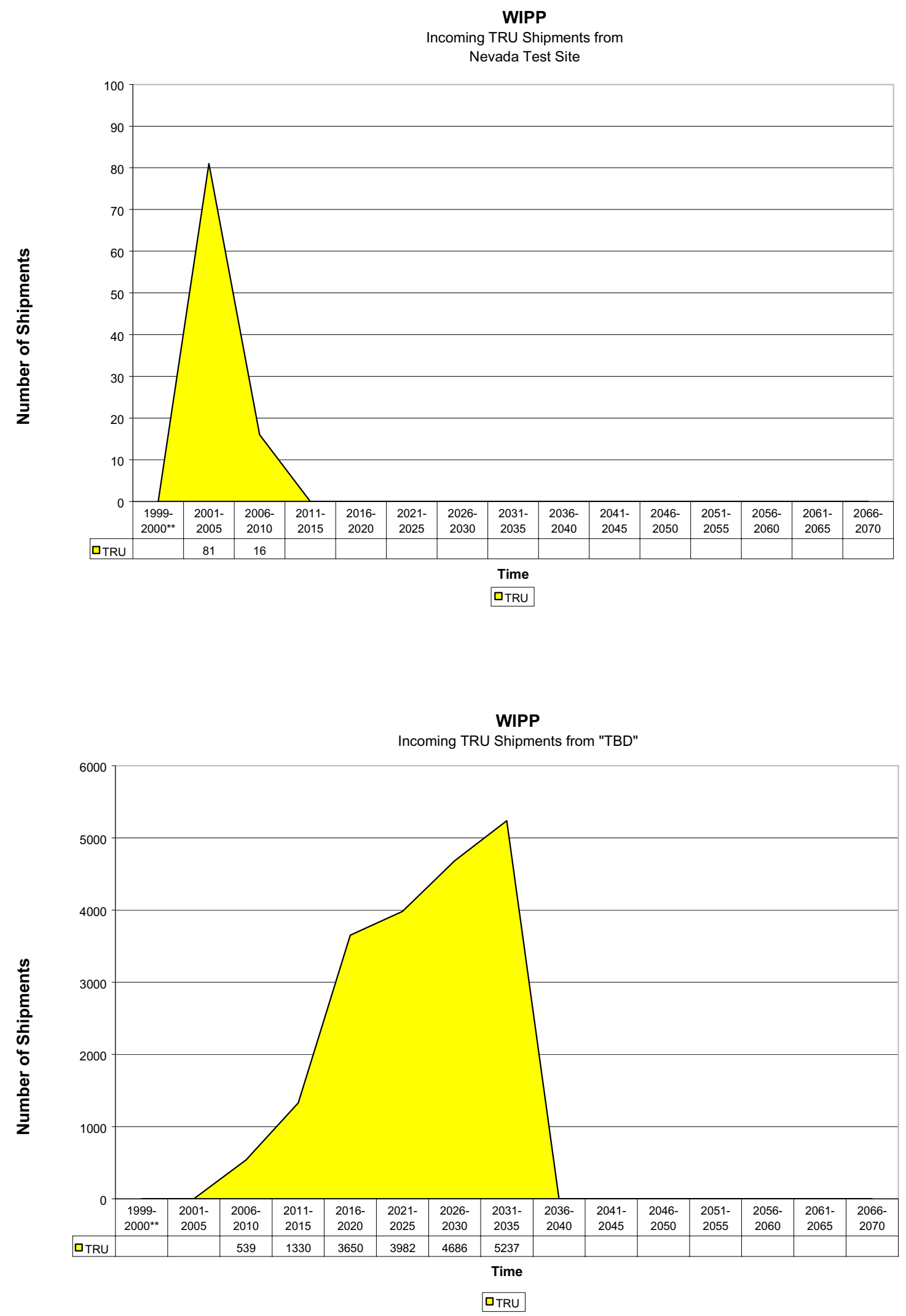

Total life-cycle values per 7/30/99 Stream Disposition Data (SDD) (http://id.inel.gov/avs/).

** Represents data for a two year period. 
WIPP

Incoming TRU Shipments from

Savannah River Site

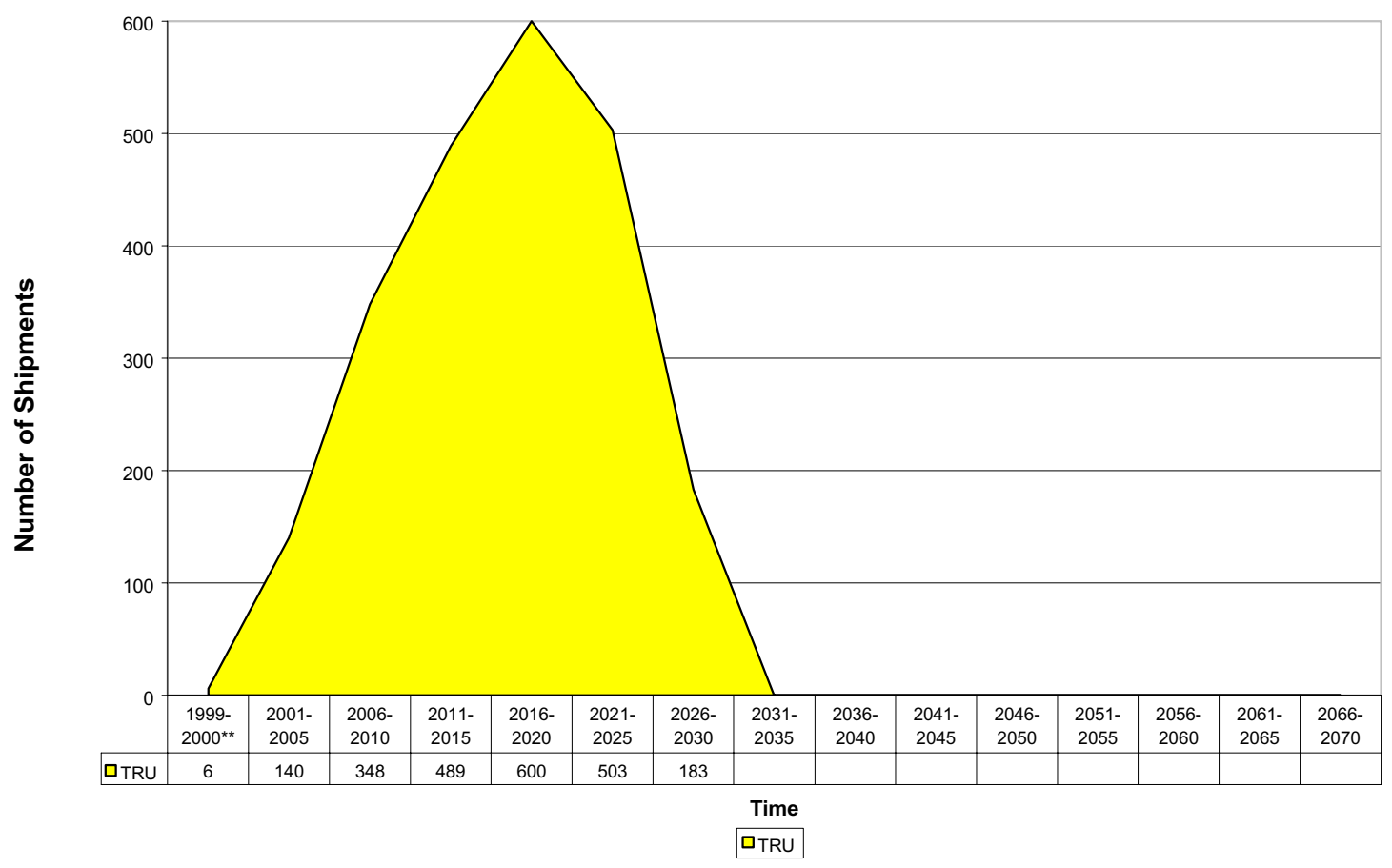

WIPP

Incoming TRU Shipments from Oak Ridge

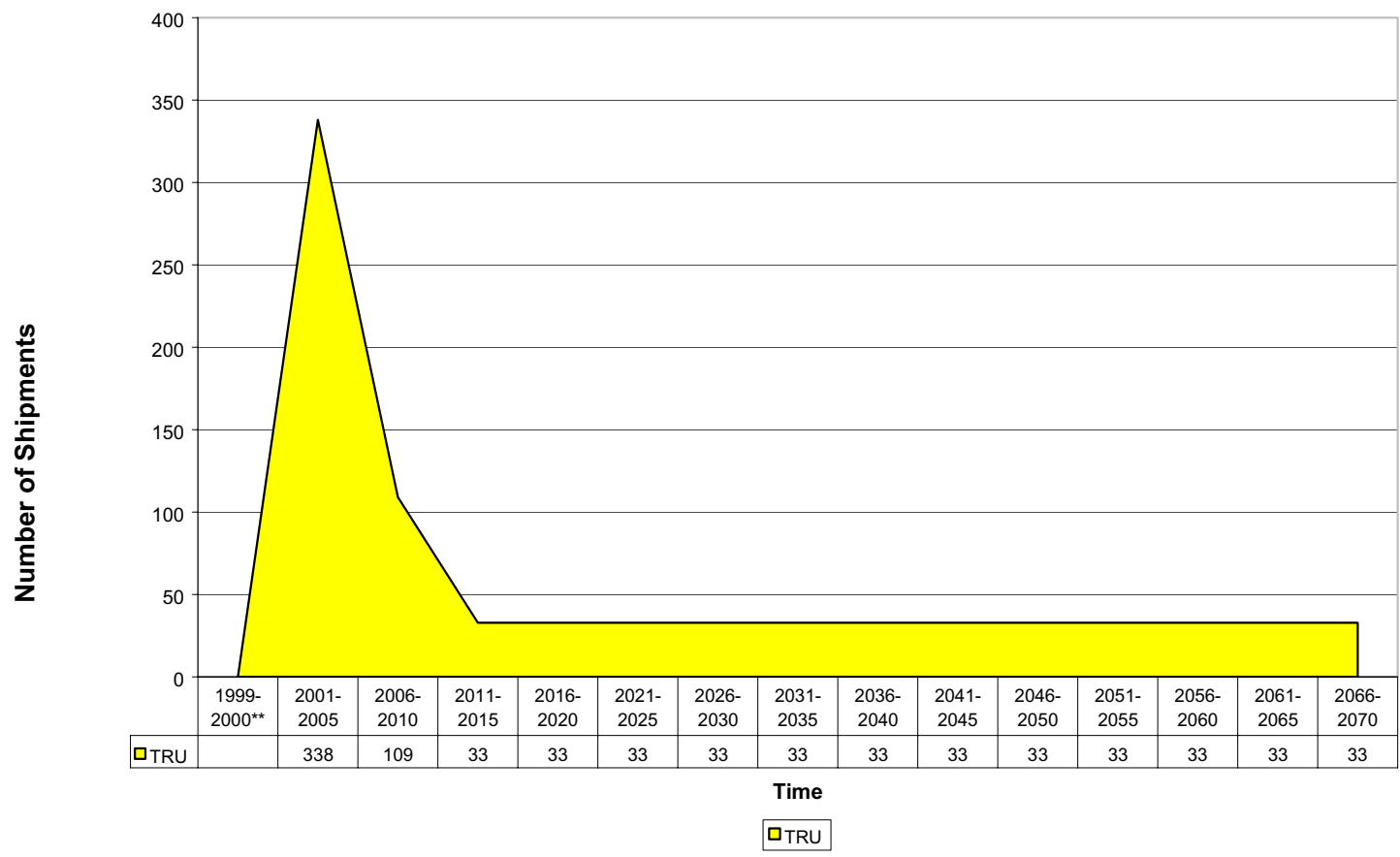

Total life-cycle values per 7/30/99 Stream Disposition Data (SDD) (http://id.inel.gov/avs/).

** Represents data for a two year period. 


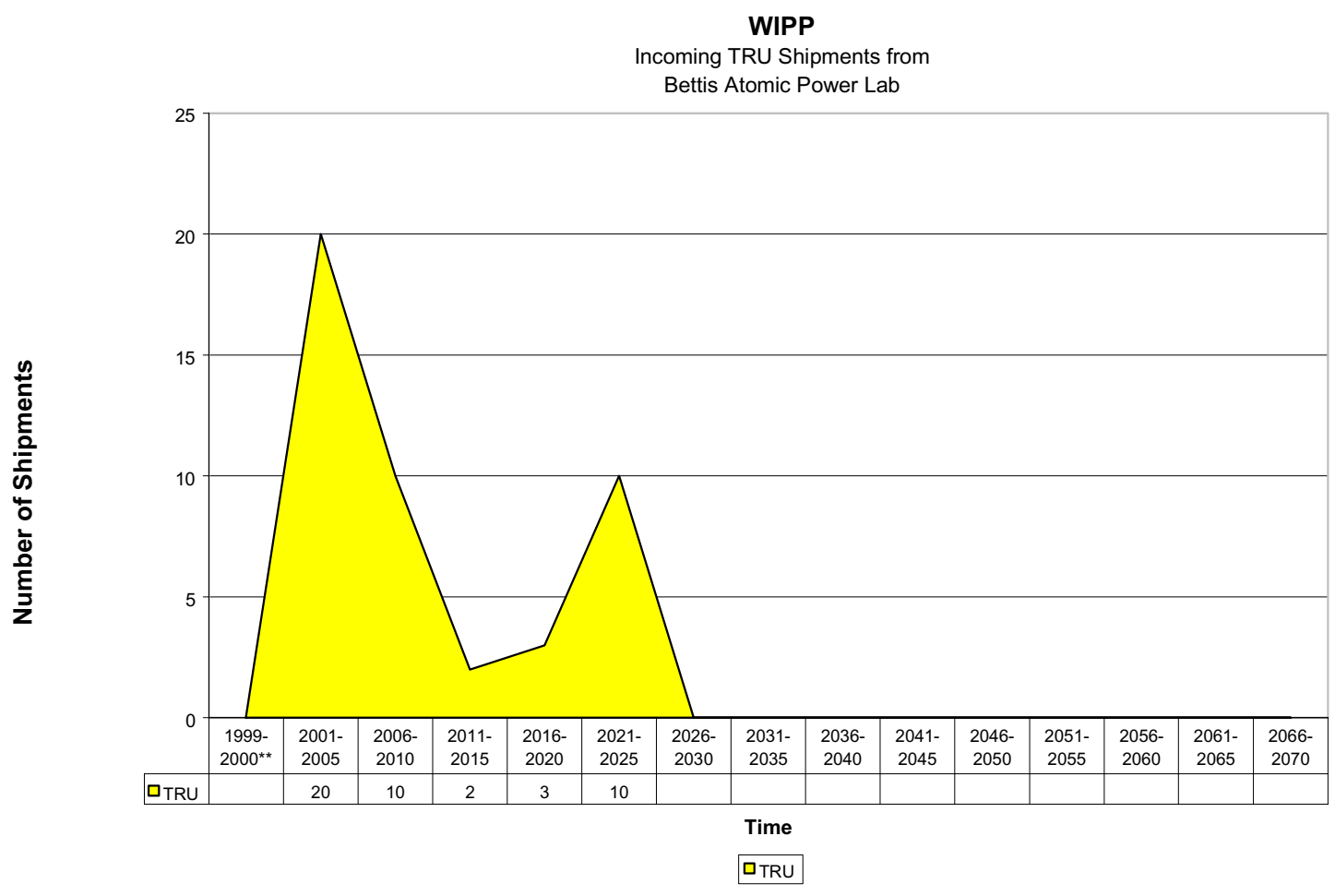

WIPP

Incoming TRU Shipments from Miamisburg Environmental Management Project

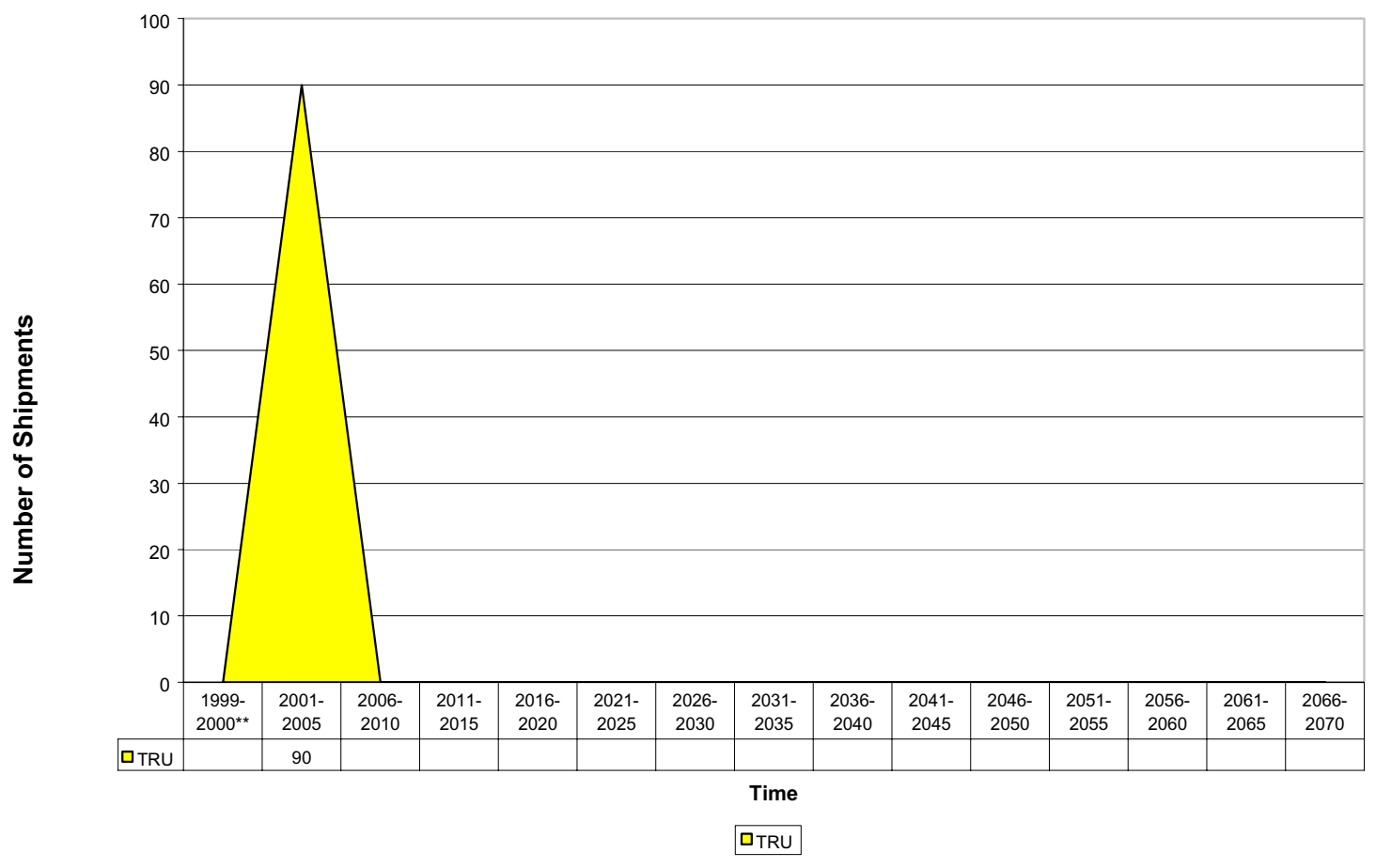

Total life-cycle values per 7/30/99 Stream Disposition Data (SDD) (http://id.inel.gov/avs/).

** Represents data for a two year period. 
WIPP

Waste Stream Disposition

Life-cycle Incoming Volume

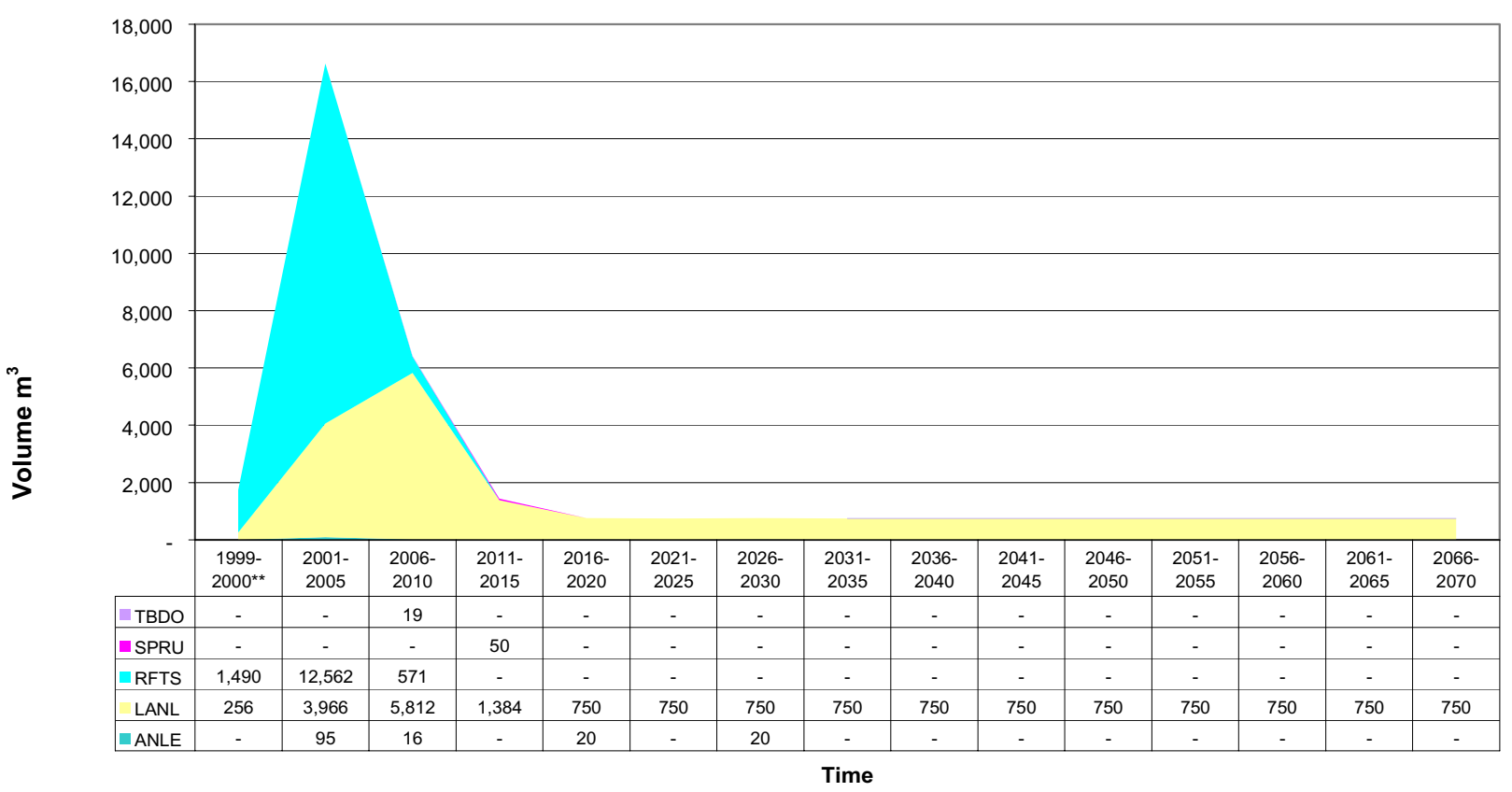

$\square$ ANLE $\quad$ LANL $\square$ RFTS $\square$ SPRU $\backsim$ TBDO

WIPP

Incoming TRU Volume from Argonne National Laboratory East

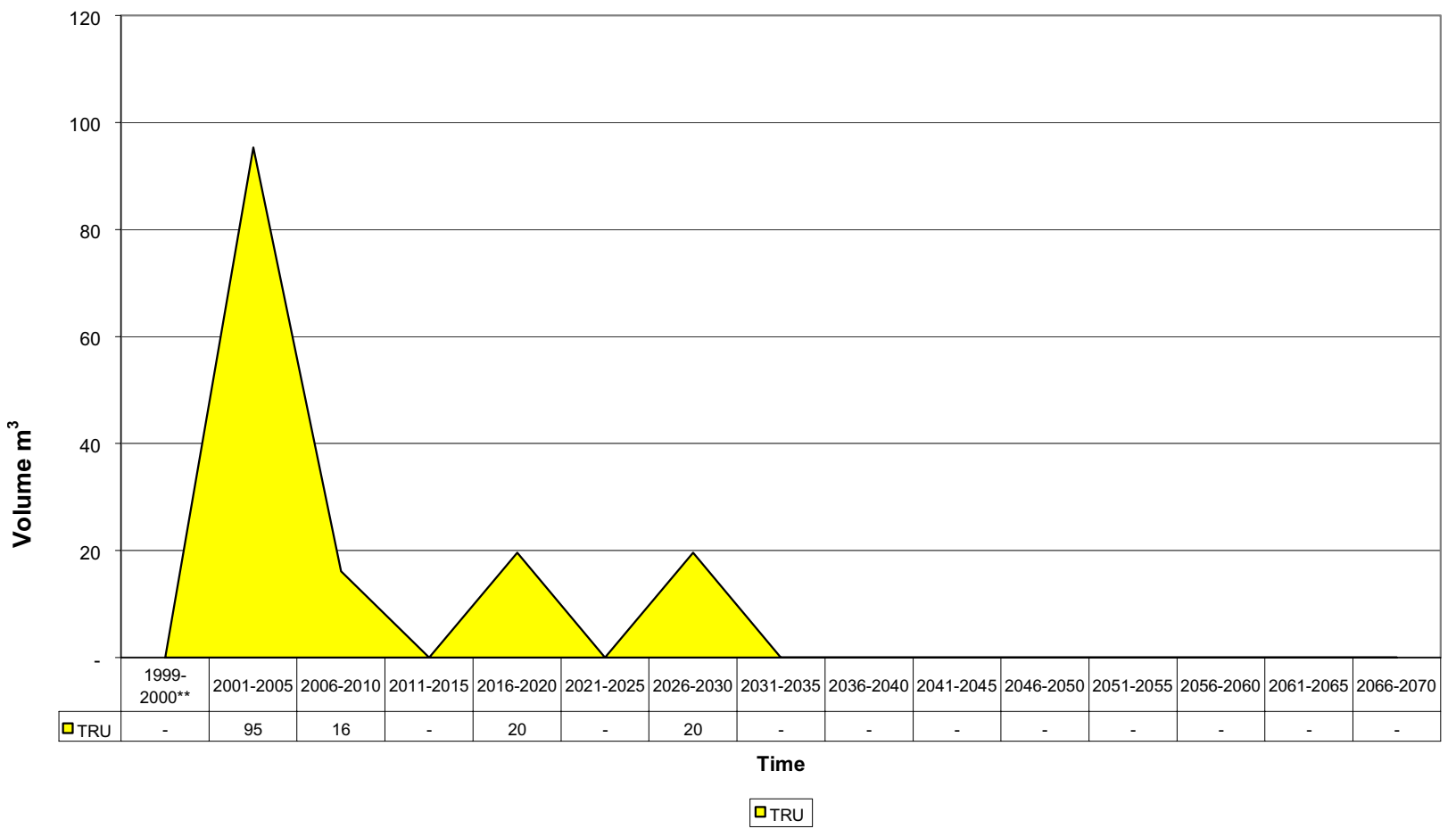

Total life-cycle values per 7/30/99 Stream Disposition Data (SDD) (http://id.inel.gov/avs/).

A-121

Printed: March 30, 2000

** Represents data for a two year period. 
WIPP

Incoming TRU Volume from Los Alamos National Laboratory

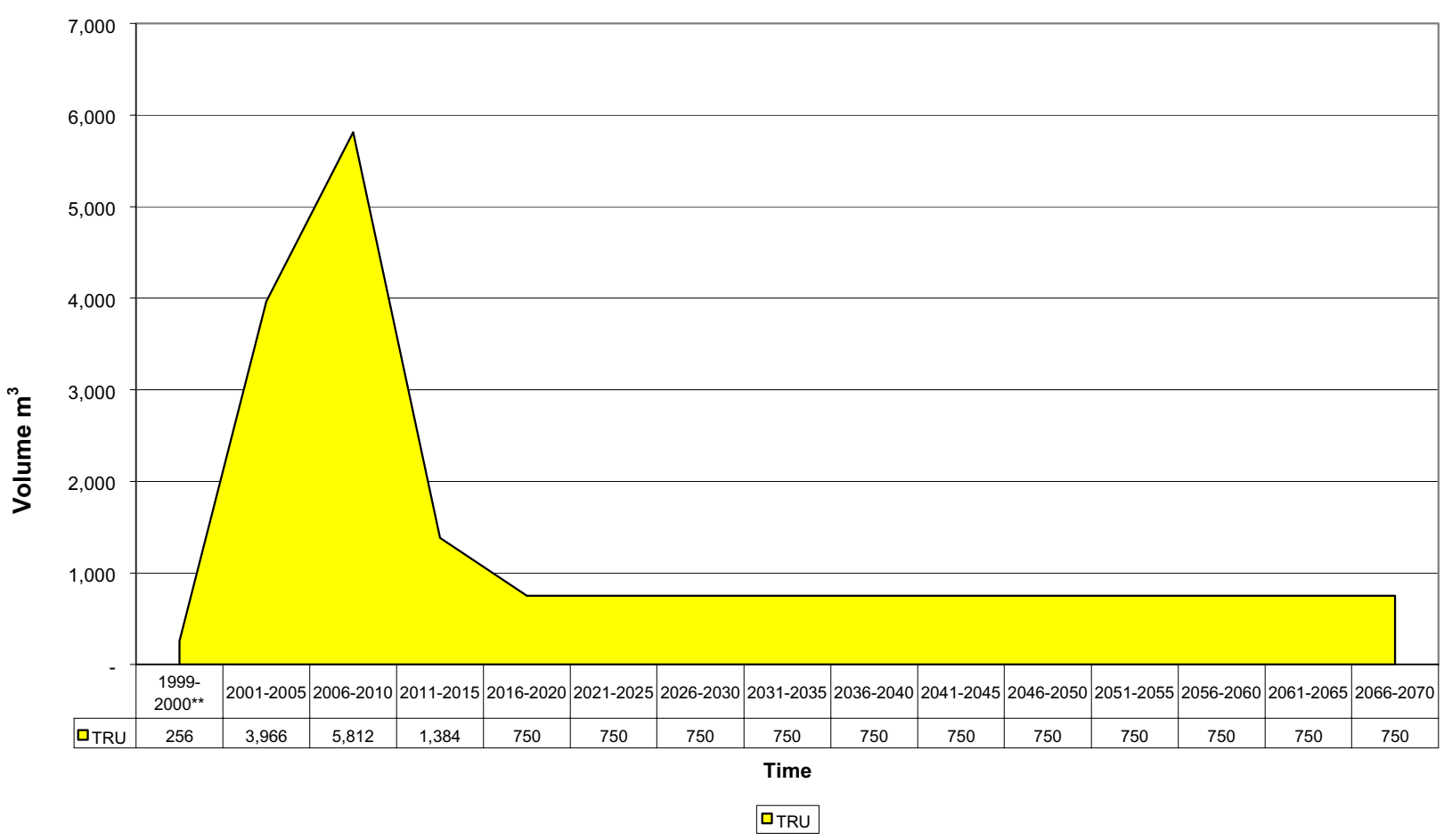

WIPP

Incoming TRU Volume from Rocky Flats

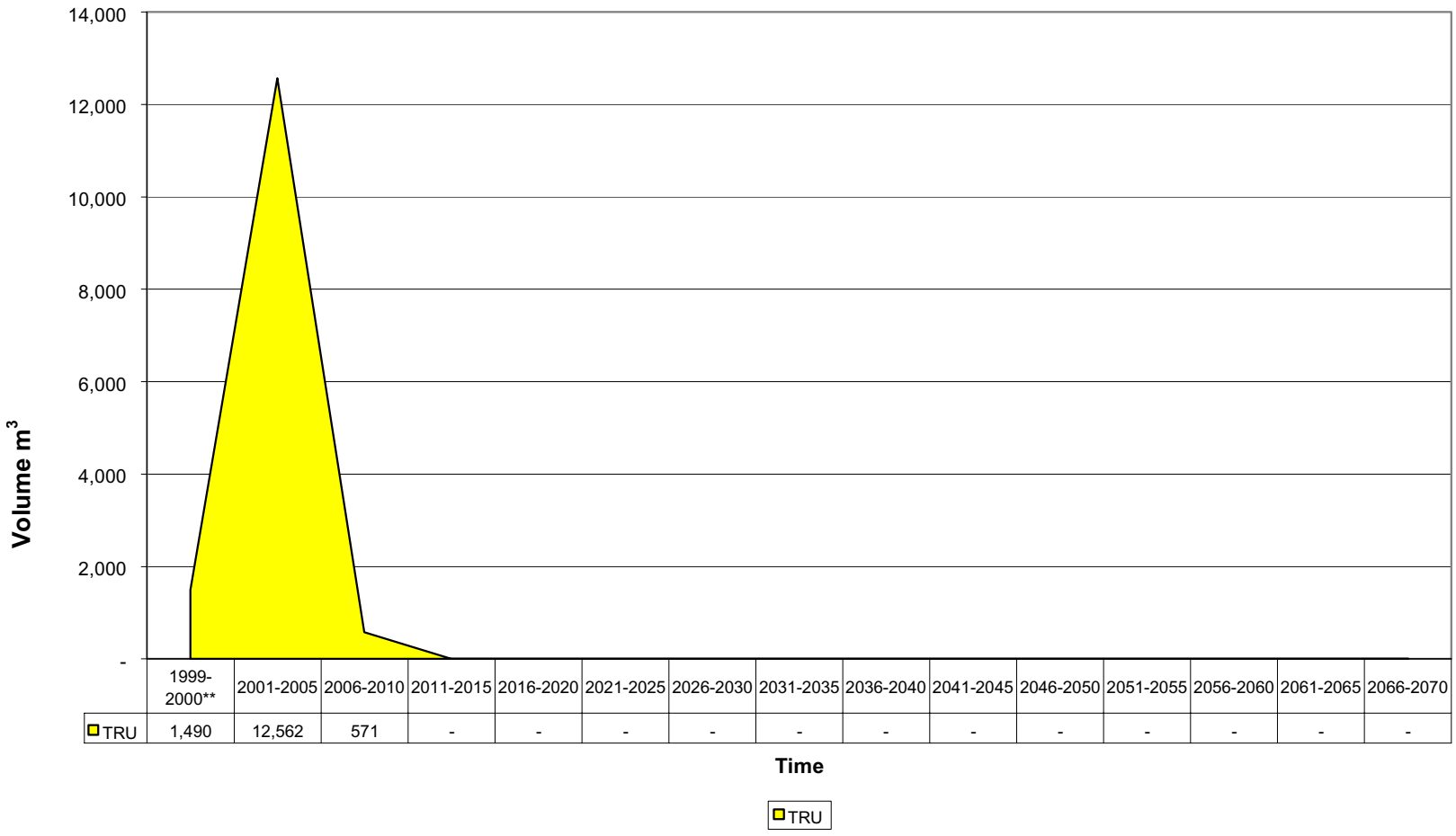

Total life-cycle values per 7/30/99 Stream Disposition Data (SDD) (http://id.inel.gov/avs/).

A-122

** Represents data for a two year period. 
WIPP

Incoming TRU Volume from Separations Process Research Unit

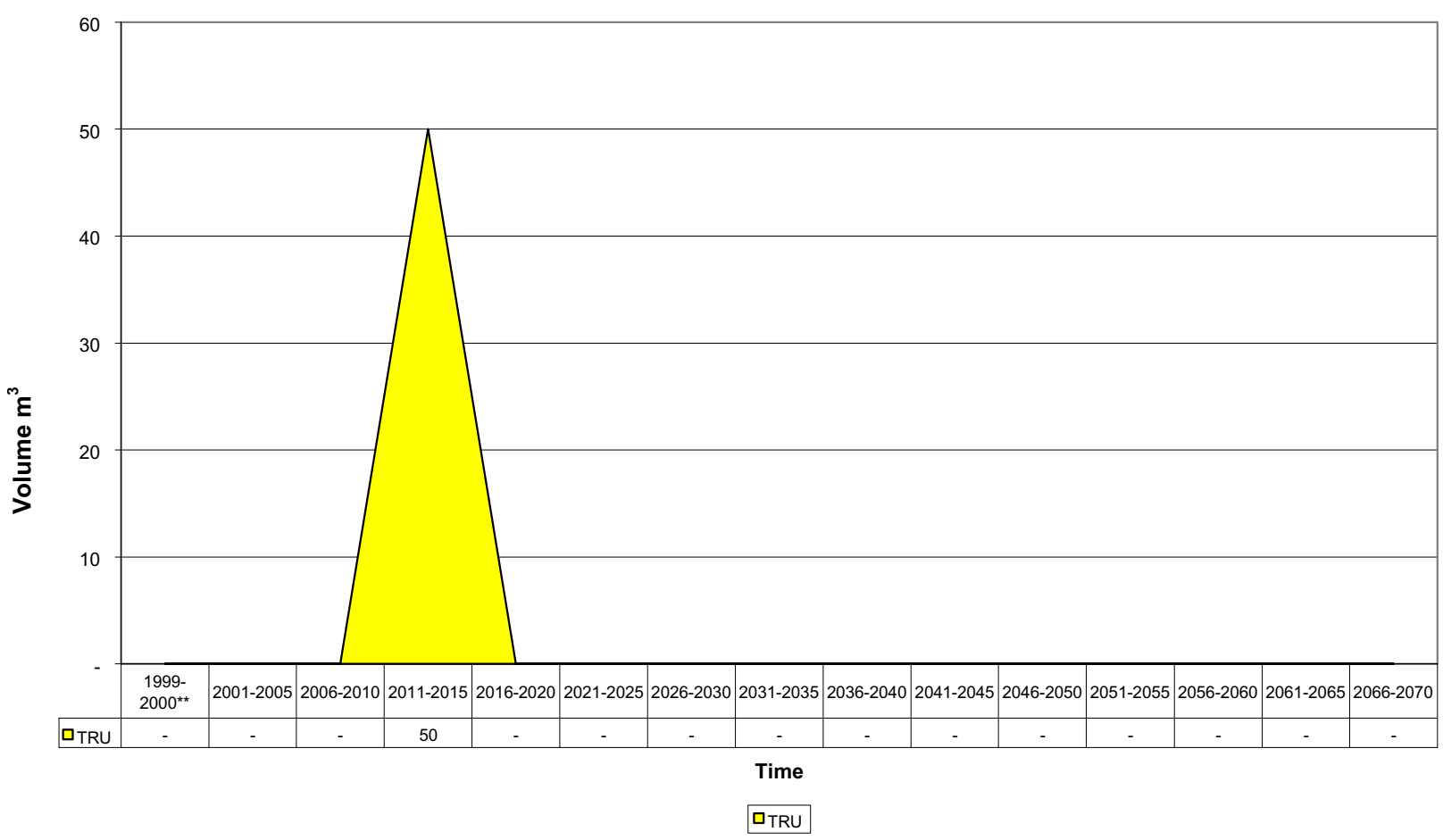

WIPP

Incoming TRU Volume from "TBDO"

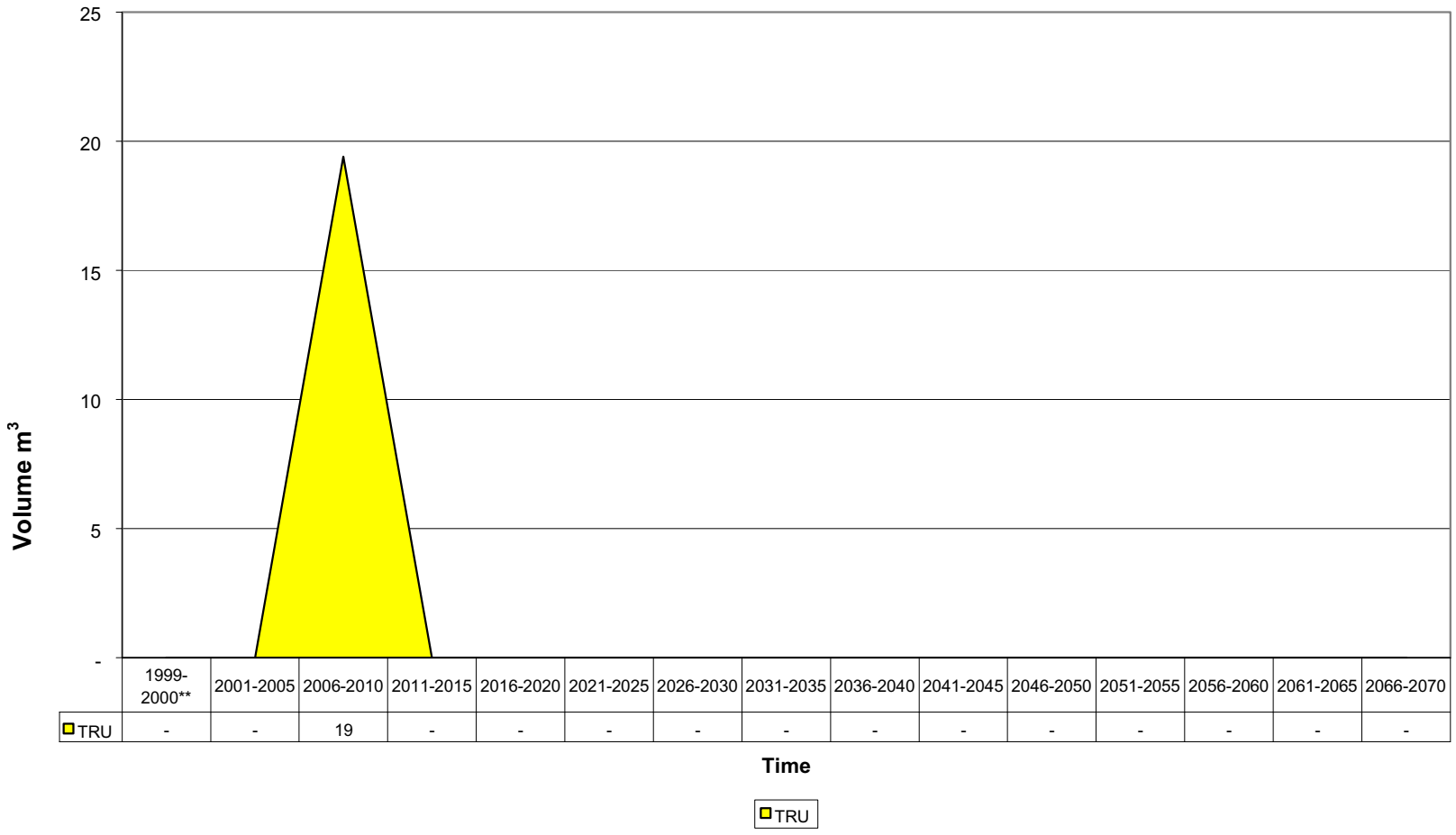

Total life-cycle values per 7/30/99 Stream Disposition Data (SDD) (http://id.inel.gov/avs/).

A-123

Printed: March 30, 2000

** Represents data for a two year period. 



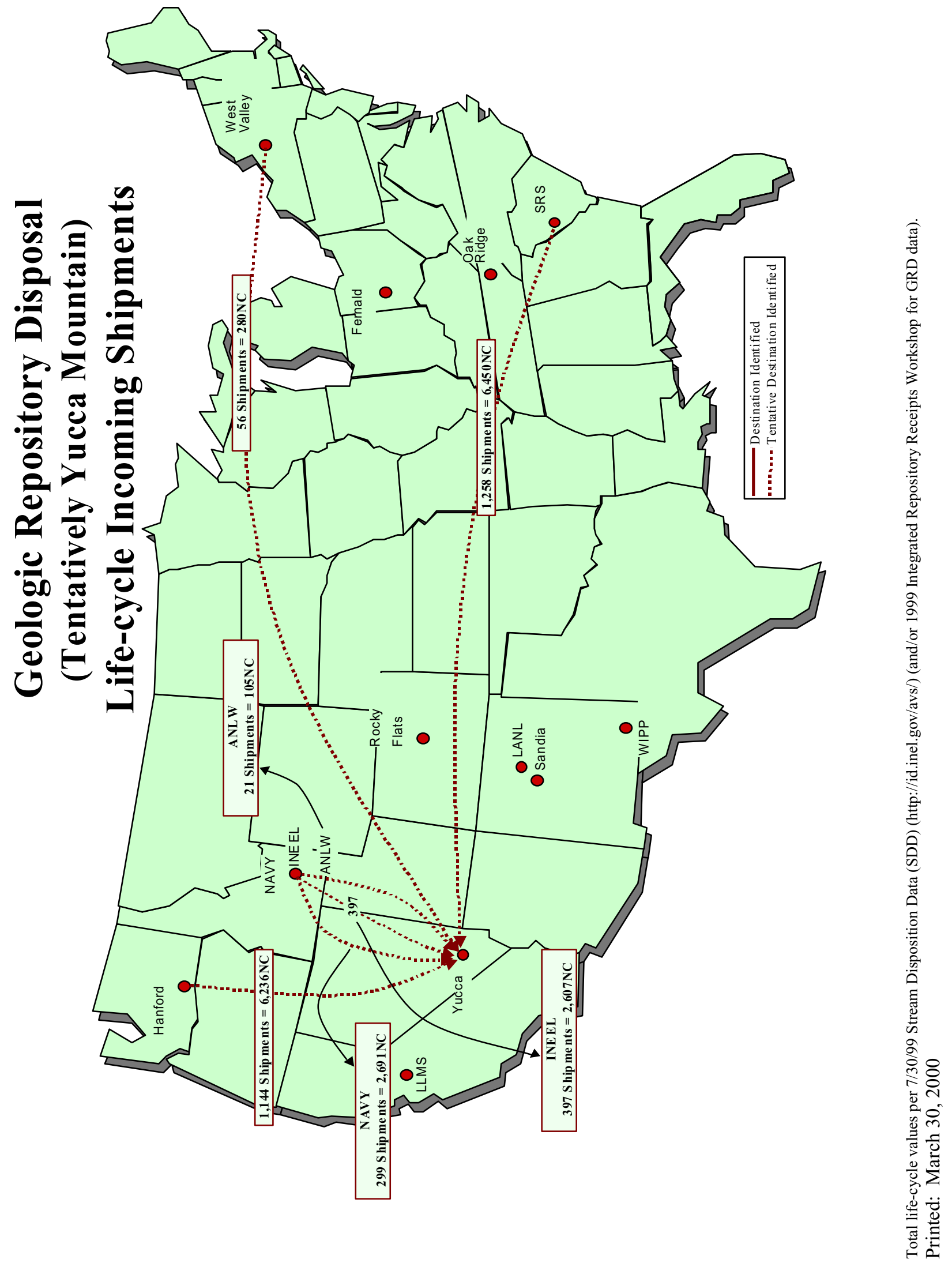


Geologic Repository Disposal

(Tentatively Yucca Mountain)

Waste Stream Disposition

Life-cycle Incoming Shipments

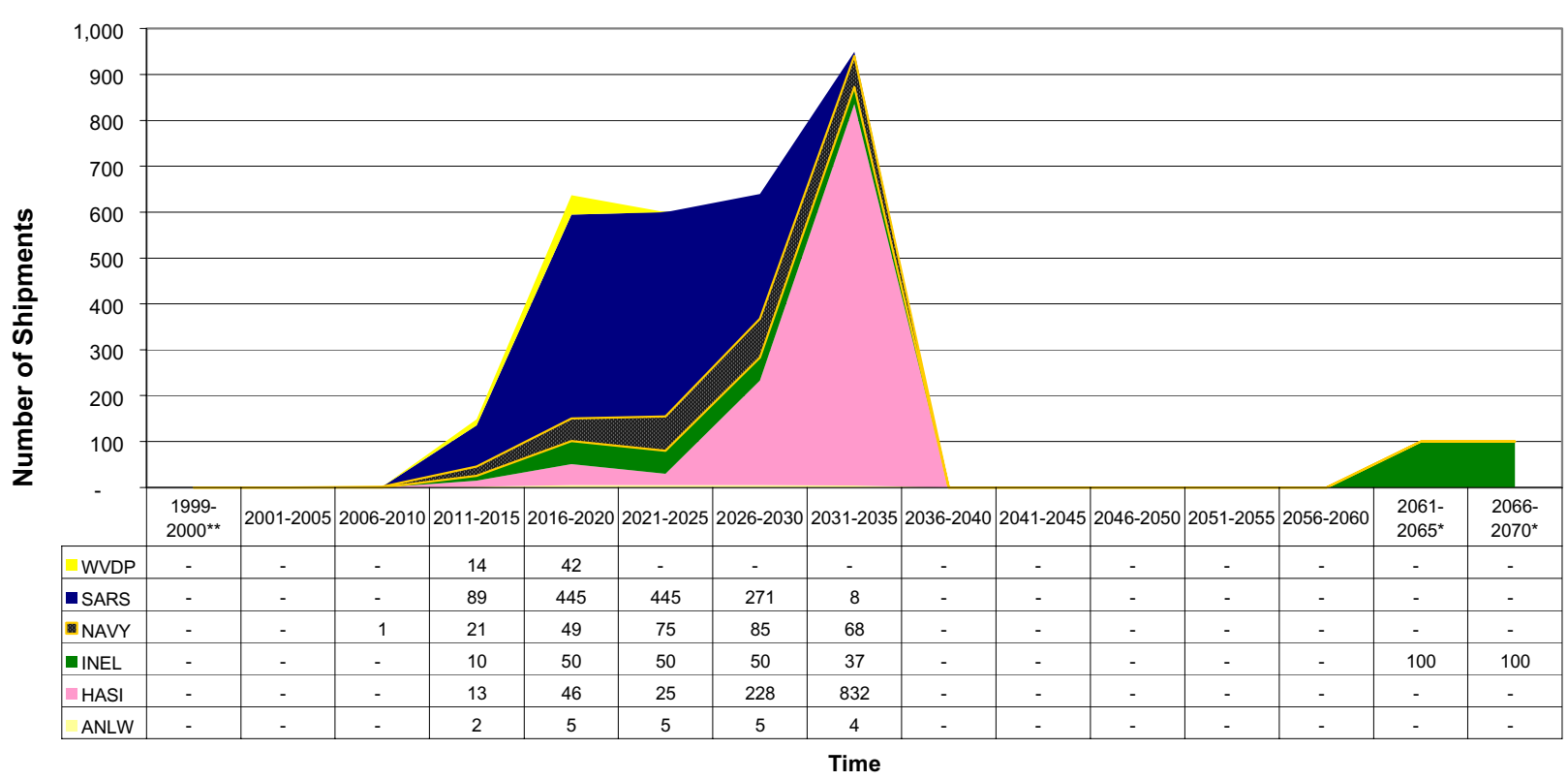

ANLW $\square$ HASI $\square I N E L=$ NAVY $\square$ SARS $\square$ WVDP

\section{Geologic Repository Disposal}

(Tentatively Yucca Mountain)

Incoming HLW Shipments from Argonne National Laboratory West

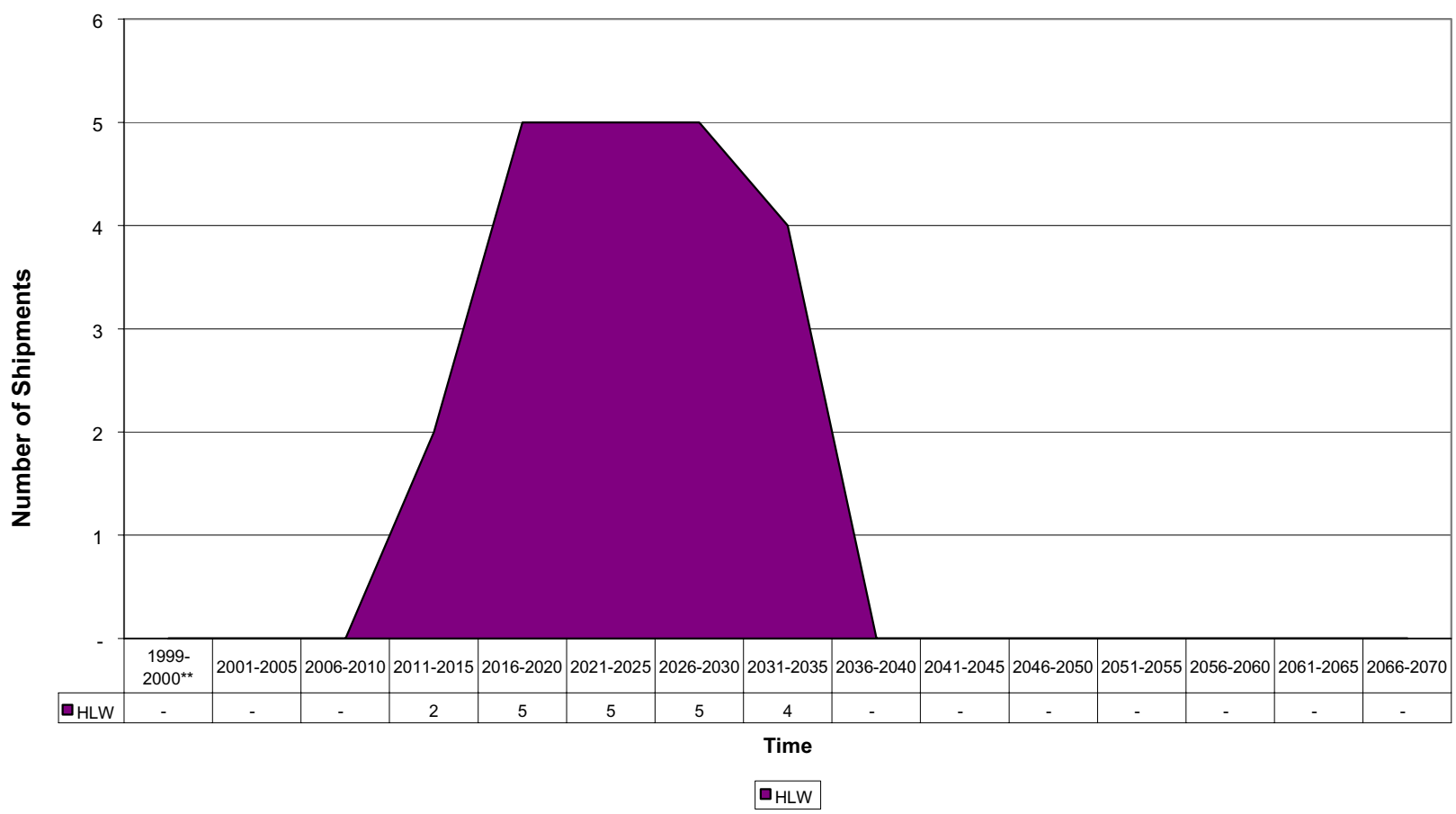

Total life-cycle values per 7/30/99 Stream Disposition Data (SDD) (http://id.inel.gov/avs/)

(and/or 1999 Integrated Repository Receipts Workshop for GRD data). Printed: March 30, 2000

** Represents data for a two year period. 
Geologic Repository Disposal

(Tentatively Yucca Mountain)

Incoming HLW/SNF Shipments from Hanford

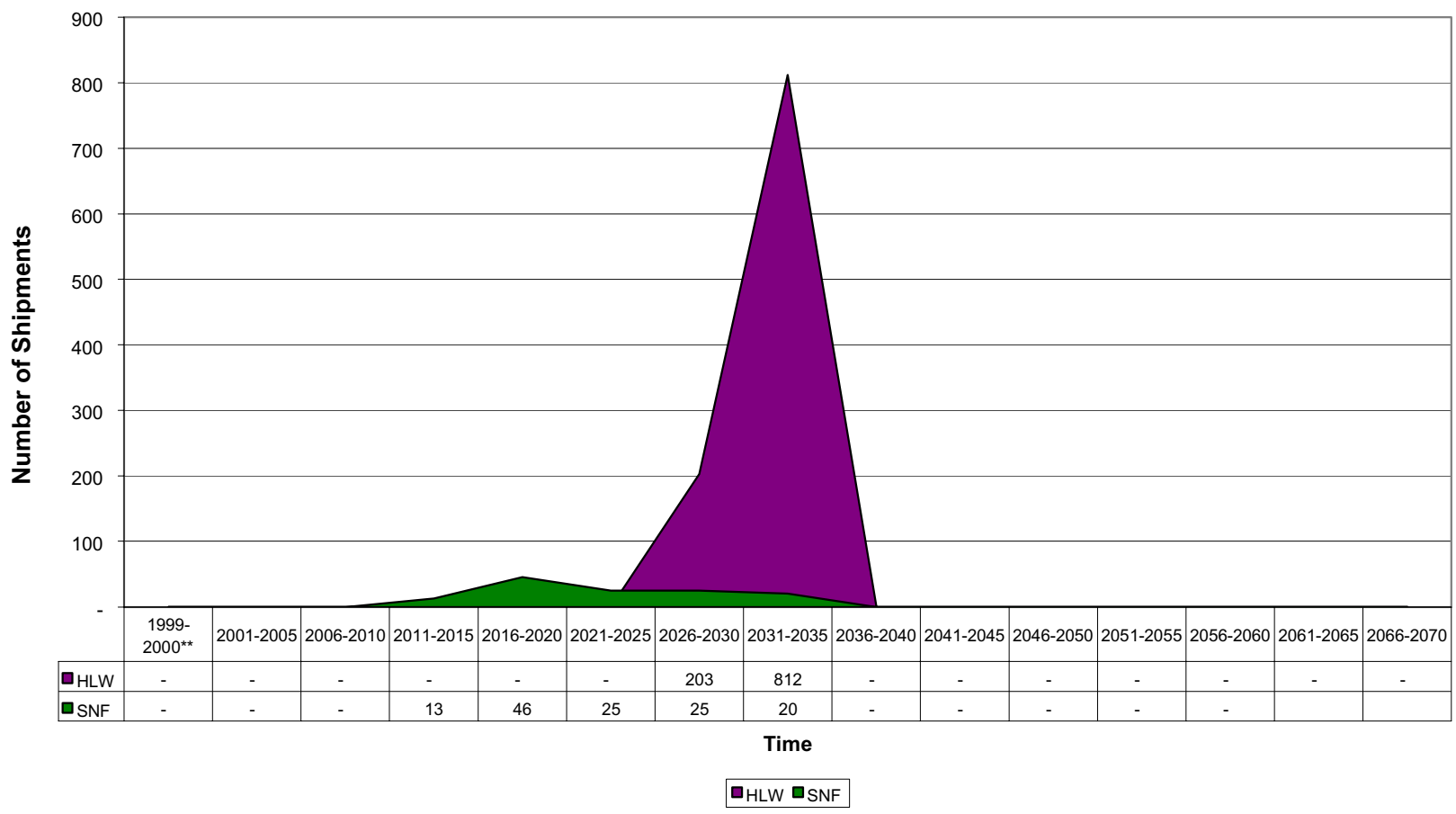

Geologic Repository Disposal

(Tentatively Yucca Mountain)

Incoming HLW/SNF Shipments from INEEL

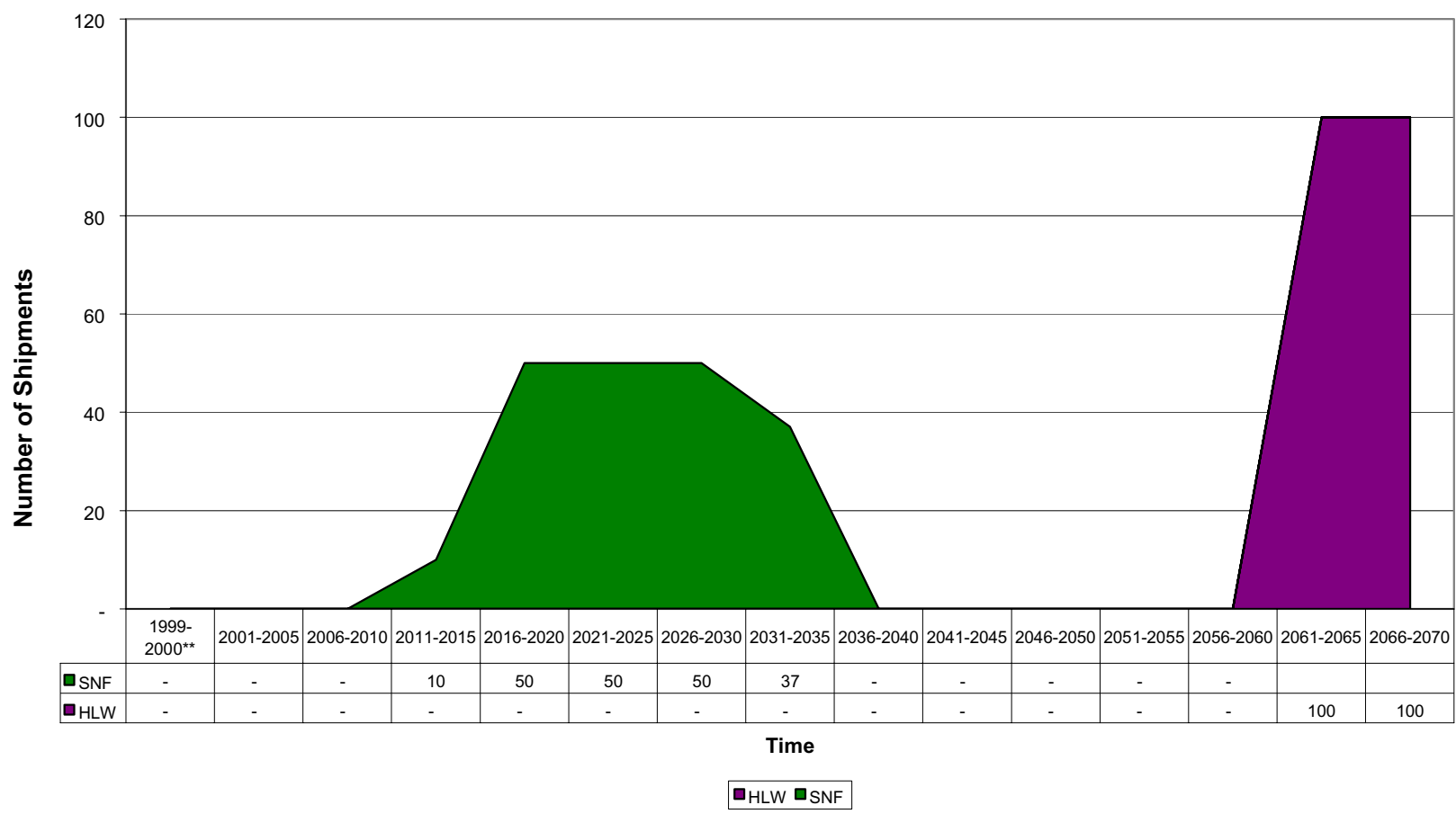

Total life-cycle values per 7/30/99 Stream Disposition Data (SDD) (http://id.inel.gov/avs/)

(and/or 1999 Integrated Repository Receipts Workshop for GRD data). Printed: March 30, 2000

** Represents data for a two year period. 


\section{Geologic Repository Disposal}

(Tentatively Yucca Mountain)

Incoming SNF Shipments from NAVY

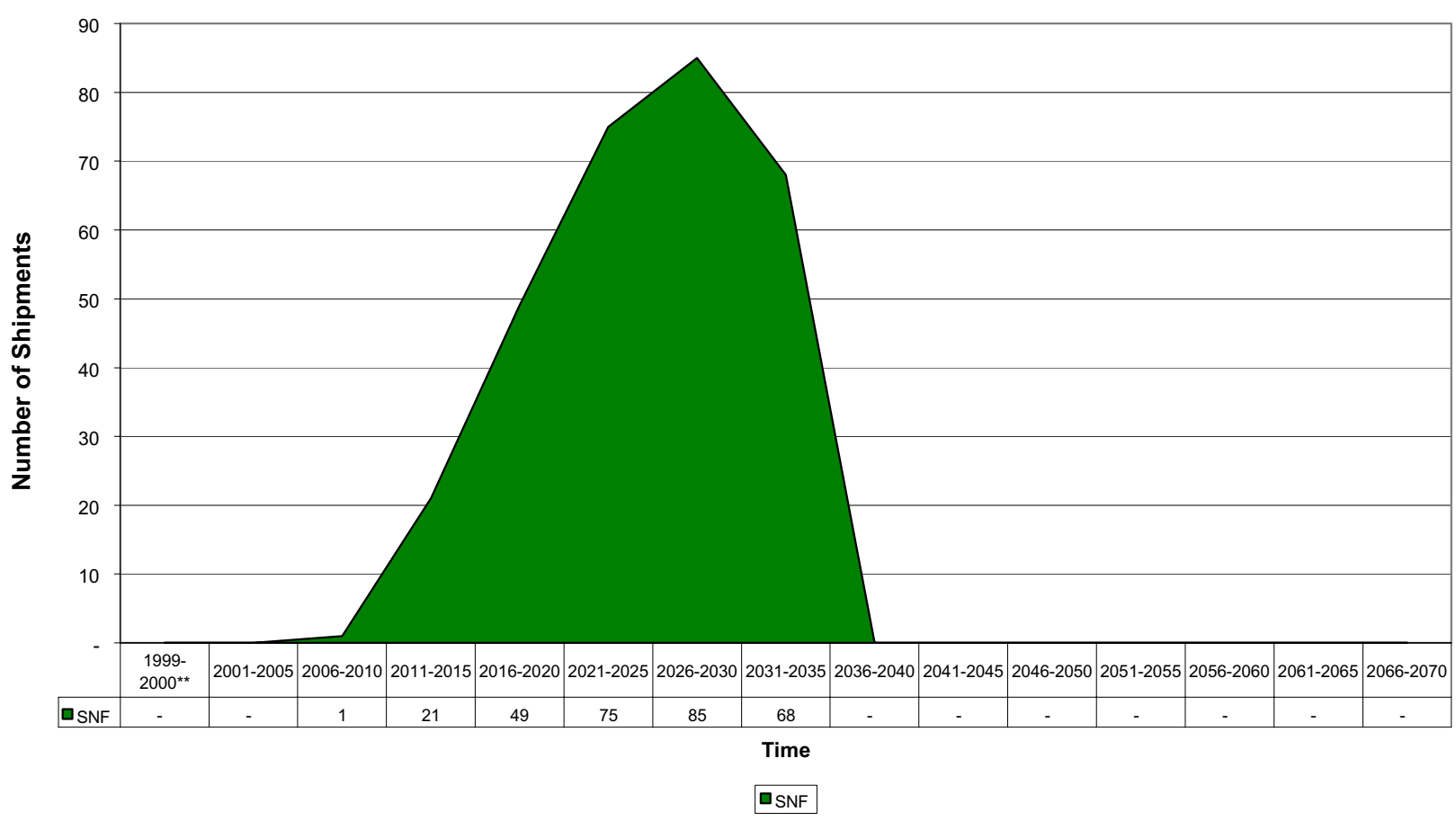

Geologic Repository Disposal

(Tentatively Yucca Mountain)

Incoming HLW/SNF Shipments from Savannah River Site

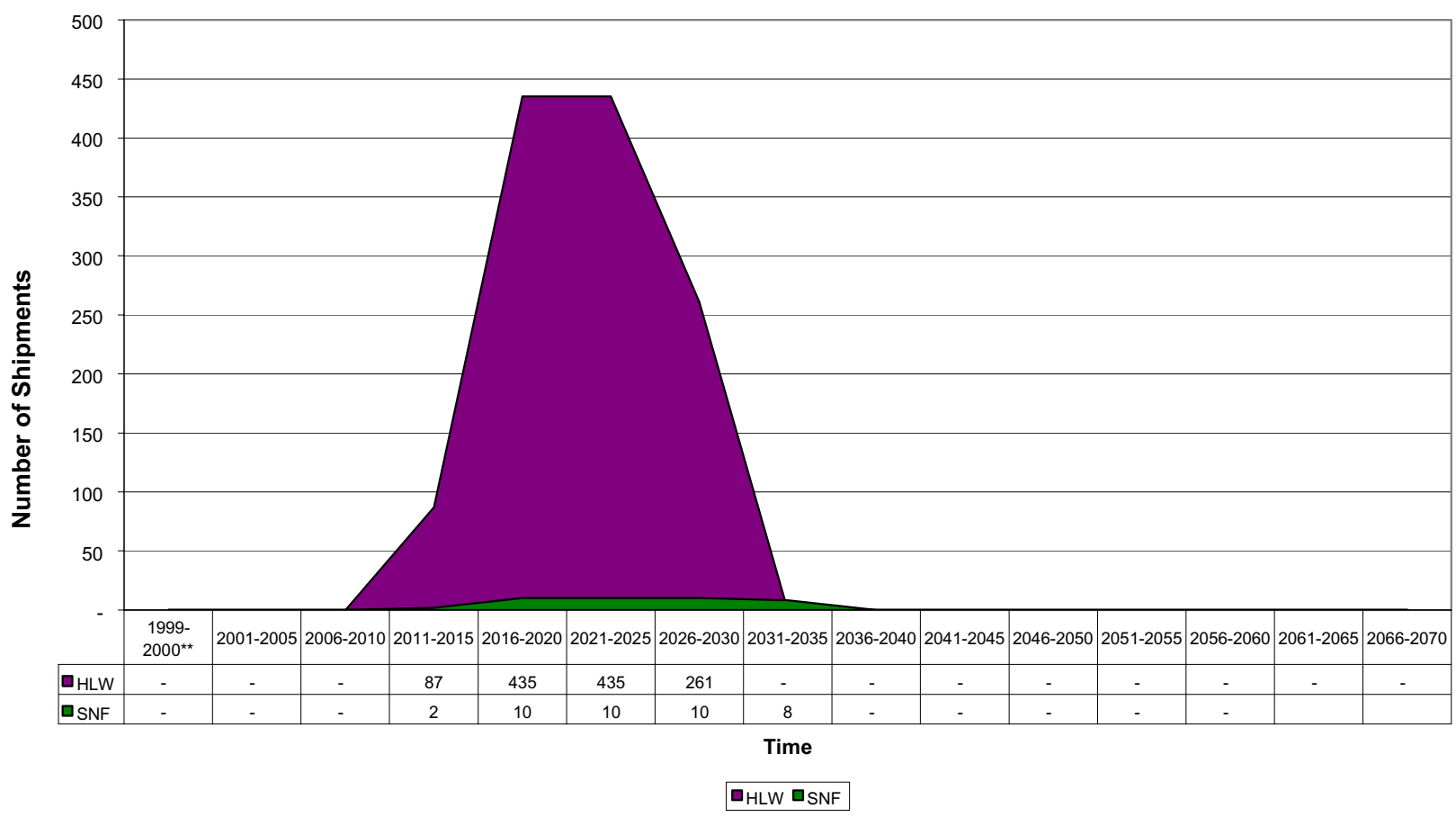

Total life-cycle values per 7/30/99 Stream Disposition Data (SDD) (http://id.inel.gov/avs/)

(and/or 1999 Integrated Repository Receipts Workshop for GRD data). Printed: March 30, 2000

** Represents data for a two year period. 


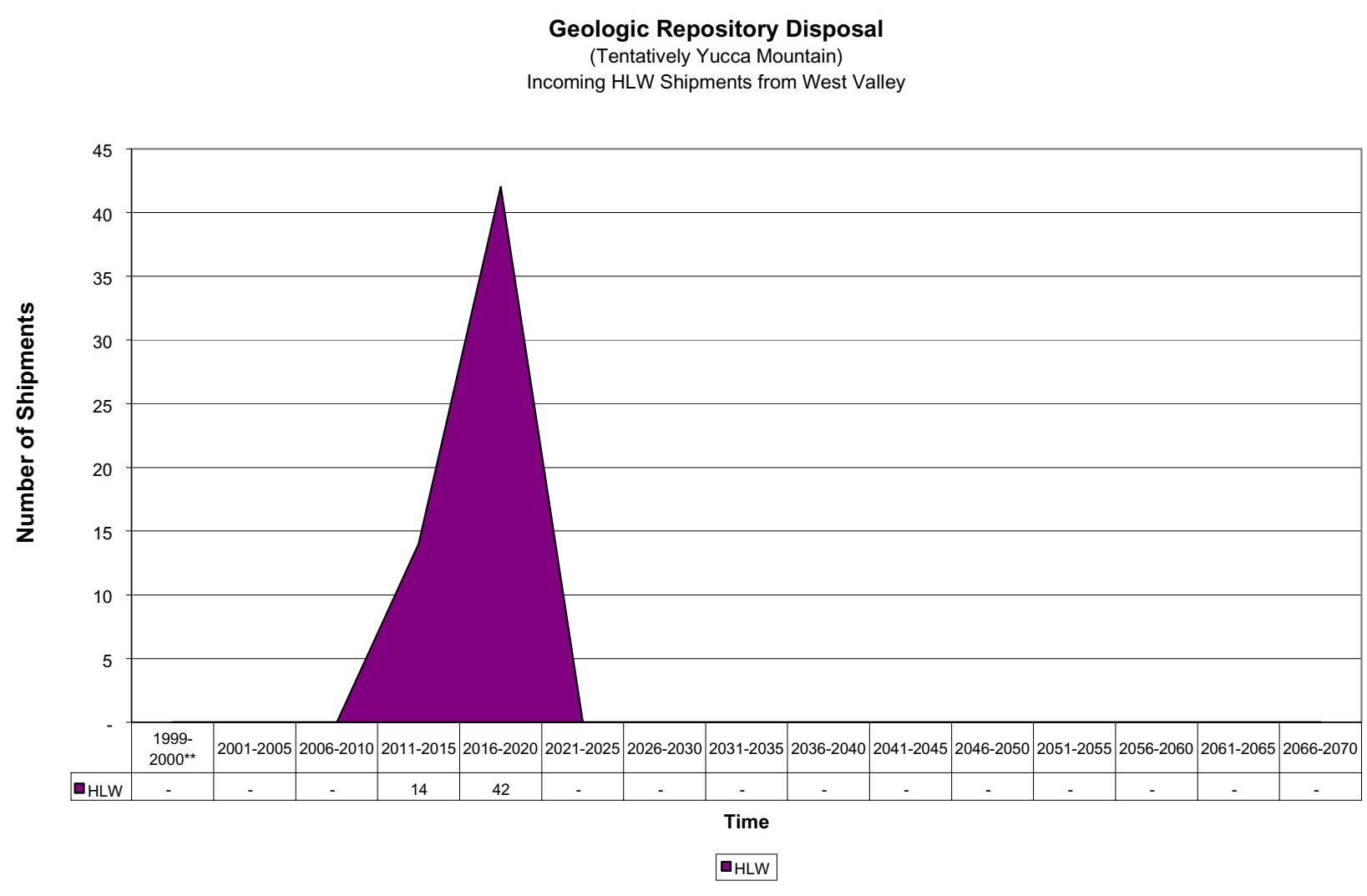

Total life-cycle values per 7/30/99 Stream Disposition Data (SDD) (http://id.inel.gov/avs/) (and/or 1999 Integrated Repository Receipts Workshop for GRD data). Printed: March 30, 2000 ** Represents data for a two year period. 




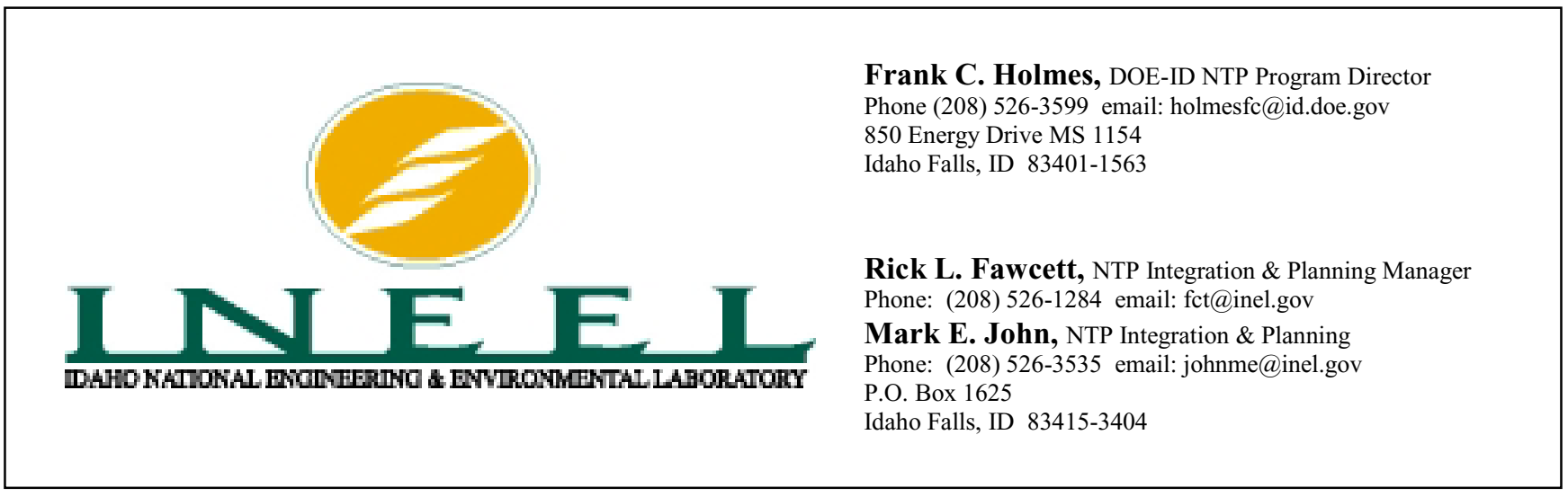

